\title{
Fibers add flavor. Part I. Classification of 5d SCFTs, flavor symmetries and BPS states
}

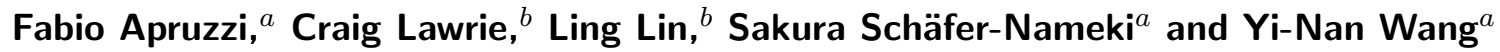 \\ ${ }^{a}$ Mathematical Institute, University of Oxford, \\ Andrew-Wiles Building, Woodstock Road, Oxford, OX2 6GG, U.K. \\ ${ }^{b}$ Department of Physics and Astronomy, University of Pennsylvania, \\ Philadelphia, PA 19104, U.S.A.
}

ABSTRACT: We propose a graph-based approach to $5 \mathrm{~d}$ superconformal field theories (SCFTs) based on their realization as M-theory compactifications on singular elliptic Calabi-Yau threefolds. Field-theoretically, these 5d SCFTs descend from $6 \mathrm{~d} \mathcal{N}=(1,0)$ SCFTs by circle compactification and mass deformations. We derive a description of these theories in terms of graphs, so-called Combined Fiber Diagrams, which encode salient features of the partially resolved Calabi-Yau geometry, and provides a combinatorial way of characterizing all 5d SCFTs that descend from a given 6d theory. Remarkably, these graphs manifestly capture strongly coupled data of the 5d SCFTs, such as the superconformal flavor symmetry, BPS states, and mass deformations. The capabilities of this approach are demonstrated by deriving all rank one and rank two 5d SCFTs. The full potential, however, becomes apparent when applied to theories with higher rank. Starting with the higher rank conformal matter theories in $6 \mathrm{~d}$, we are led to the discovery of previously unknown flavor symmetry enhancements and new 5d SCFTs.

KEywords: Conformal Field Models in String Theory, F-Theory, M-Theory, Supersymmetry and Duality

ARXIV EPRINT: 1907.05404 


\section{Contents}

1 Introduction 1

1.1 Strategy 6

2 M-theory, 5d SCFTs, and resolutions of elliptic fibrations 9

2.1 5d SCFTs, gauge theories, and Coulomb branches 9

2.2 Geometric engineering of 5d gauge theories and SCFTs 11

$\begin{array}{lll}2.3 & \text { SCFTs and flavor from geometry } & 14\end{array}$

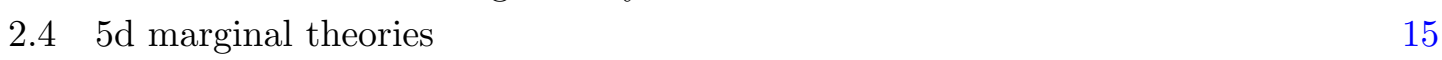

$\begin{array}{ll}2.5 & \text { Singular elliptic Calabi-Yau threefolds } \\ & 16\end{array}$

$\begin{array}{ll}\text { 2.5.1 Codimension one: Kodaira fibers } & 17\end{array}$

$\begin{array}{ll}\text { 2.5.2 Codimension two: flat and non-flat resolutions } & 17\end{array}$

\begin{tabular}{ll}
2.5 .3 & Resolution of singular fibers \\
\hline
\end{tabular}

3 5d SCFTs from conformal matter theories $\quad 21$

3.1 M-theory on non-flat fibrations: a dictionary 22

3.2 Rank one classification from non-flat resolutions 24

$\begin{array}{lll}3.3 & \text { Rank two: non-flat resolutions } & 28\end{array}$

3.3.1 Non-flat resolutions of $\left(\mathrm{E}_{8}, \mathrm{SU}(2)\right) \quad 29$

3.3.2 Non-flat resolutions of $\left(D_{10}, I_{1}\right) \quad 31$

3.4 Higher rank conformal matter 33

4 5d SCFTs from graphs $\quad \mathbf{3 5}$

4.1 Combined Fiber Diagrams (CFDs) 35

$\begin{array}{lll}4.2 & \text { Starting points: marginal CFDs } & 38\end{array}$

4.3 Rank one classification from CFDs 40

4.4 Rank two classification from CFDs 40

4.4.1 Marginal CFDs from automorphisms 42

4.4.2 New flavor symmetry predictions for rank two theories 47

$4.5\left(\mathrm{E}_{n}, \mathrm{E}_{n}\right)$ minimal conformal matter theories 48

4.6 Higher rank: $\left(\mathrm{E}_{8}, \mathrm{SU}(n)\right) \quad 50$

5 BPS states $\quad \mathbf{5 4}$

5.1 BPS states from wrapped M2-branes 54

$\begin{array}{lll}5.2 & \text { BPS states of rank one SCFTs } & 57\end{array}$

$\begin{array}{lll}5.3 & \text { BPS states of rank two SCFTs } & 57\end{array}$

6 Conclusions and outlook $\quad 59$

$\begin{array}{ll}\text { A Summary for rank one and two 5d SCFTs } & \mathbf{6 1}\end{array}$

A.1 Marginal theories in 5d 61

A.1.1 Marginal theories for rank one SCFTs 62

A.1.2 Marginal theories for rank two SCFTs 62

A.2 Summary tables for rank one and rank two 5d SCFTs 66 
$\begin{array}{ll}\text { B Rank two prepotentials } & 66\end{array}$

$\begin{array}{ll}\text { C Rational surfaces: } \mathrm{dP} \text { versus gdP } & \mathbf{7 6}\end{array}$

$\begin{array}{ll}\text { D Details of non-flat resolutions } & \mathbf{7 8}\end{array}$

$\begin{array}{ll}\text { D.1 Non-flat fiber resolutions } & 78\end{array}$

$\begin{array}{lll}\text { D.1.1 Rank one theories } & 78\end{array}$

D.2 Blow-ups for the rank two E-string $\left(\mathrm{E}_{8}, \mathrm{SU}(2)\right) \quad 80$

$\begin{array}{lll}\text { D.2.1 Geometry for the marginal theory } & 80\end{array}$

D.2.2 Example resolutions: $\left(\mathrm{E}_{8}, \mathrm{SU}(2)\right) \quad 84$

D.3 Blow-ups for the $\left(D_{10}, I_{1}\right)$ conformal matter $\quad 90$

D.3.1 Geometry for the marginal theory 90

D.3.2 Example resolution for $\left(D_{10}, I_{1}\right) \quad 91$

D.4 Resolution of $\left(\mathrm{E}_{8}, \mathrm{SU}(3)\right) \quad 92$

D.5 Higher rank marginal theories $\quad 93$

$\begin{array}{lll}\text { D.6 } & \text { Alternative 6d starting points } & 96\end{array}$

D.6.1 Alternative description for rank one: $\left(\mathrm{E}_{6}, \mathrm{SU}(3)\right) \quad 96$

$\begin{array}{ll}\text { D.6.2 Alternative description: }\left(D_{5}, D_{5}\right) & 97\end{array}$

$\begin{array}{lll}\text { D.6.3 Flavor symmetry and BPS states } & 98\end{array}$

\section{Introduction}

Geometry is a well-established tool in the exploration of the landscape of superconformal field theories (SCFTs). This connection has by now crystalized into a profound correspondence, where geometric structures have emerged as central agents in the classification of SCFTs. Hallmarks of this achievement are the classifications of 6d SCFTs with maximal [1] and $\mathcal{N}=(1,0)[2-4]$ supersymmetry, as well as rank one and two 5d SCFTs [5, 6], and the Coulomb branch geometries for rank one $4 \mathrm{~d} \mathcal{N}=2$ theories [7]. In these considerations, the geometry not only provides a concrete realization of the SCFT in an M-/F-theory or string theory background, but, more importantly, constitutes an organizing principle by which to construct and enumerate all such theories systematically. Such classifications may come with caveats in that not all theories may have purely geometric constructions (see e.g., [3]). However, the geometric classification oftentimes results in a parallel field theoretic classification, which corroborates the completeness.

Ideally, a classification result does not only provide a formal identification of all theories in a given dimension and amount of supersymmetry, but contains information about the physics of the strongly coupled SCFTs. In this paper we propose a classification approach for 5d SCFTs, with $\mathcal{N}=1$ supersymmetry, which includes not only a systematic way of constructing the associated geometries in their realization via an M-theory compactification on a Calabi-Yau threefold, but also allows the reading off of

1. the flavor symmetry of the strongly coupled 5d SCFT, and

2. BPS states of the 5d SCFT. 


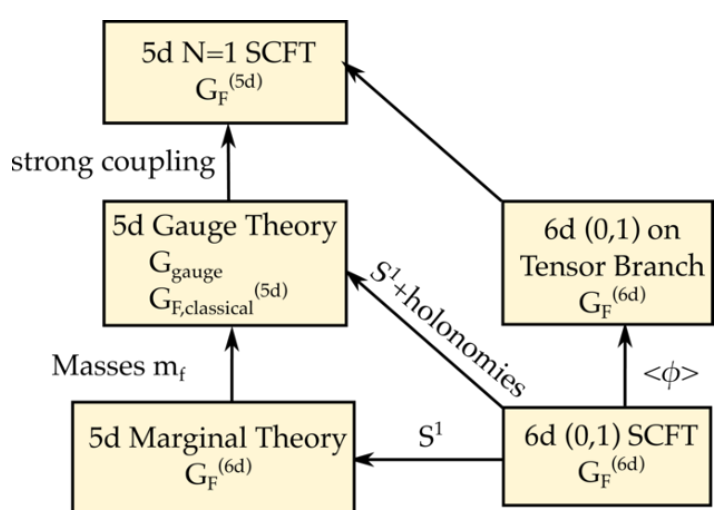

(a)

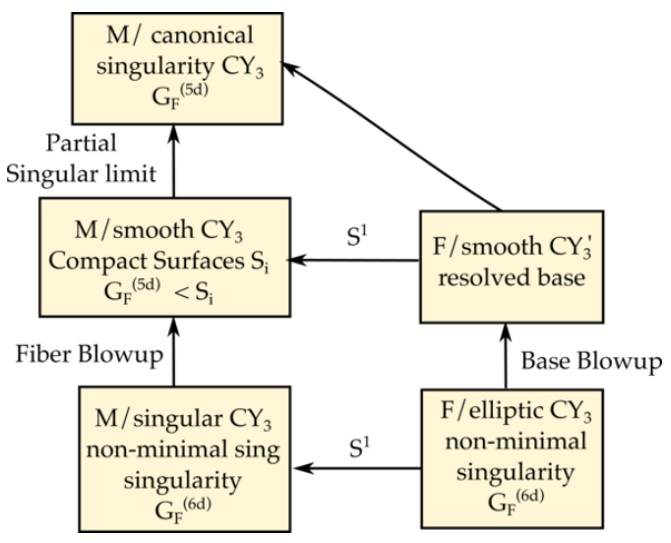

(b)

Figure 1. (a) Field-Theory overview: starting from a 6d SCFT, the circle-reduction yields the 5d marginal theory. Mass deforming the marginal theory gives rise to $5 \mathrm{~d}$ gauge theories that flows to $5 \mathrm{~d}$ SCFTs in the UV. Alternatively, one can reduce the $6 \mathrm{~d}$ theory on a circle with holonomies and then flow to said SCFTs. A third alternative is to take the 6d SCFT onto the tensor branch and reduce to 5d. (b) Geometry overview: the geometric realization in F/M-theory complements the field theoretic approach. A 6d SCFT is constructed from F-theory on a non-compact elliptically fibered Calabi-Yau threefold $\mathrm{CY}_{3}$ with non-minimal singularities. These have crepant resolutions either by blow-ups in the fiber (an approach useful e.g. for conformal matter theories) or by blow-ups that modify the base of the fibration. Each of these approaches introduces compact surfaces, $S_{i}$, and the $5 \mathrm{~d}$ strongly coupled flavor symmetry is encoded in the geometry of certain curves associated to the $6 \mathrm{~d}$ flavor symmetry inside the surfaces $S_{i}$. We propose a succinct way of tracking these, so-called flavor curves, in terms of a graphical tool, making $G_{\mathrm{F}}^{(5 \mathrm{~d})}$ that is encoded in the geometry manifest - see figure 2 .

The flavor symmetry of the SCFT will be manifestly encoded in the way we construct and present the geometries, and literally can be read off. Some BPS states can be computed applying a straightforward procedure from the geometric information that we provide. We introduced a graphical presentation of this data in terms of so-called combined fiber diagrams (CFDs) in the recent paper [8]. The current paper can be viewed as providing the geometric derivation of these CFDs.

The study of 5d SCFTs using M-theory compactifications on singular Calabi-Yau threefolds goes back to $[5,9]$ and was recently revisited in $[6,10-14]$. This complements the approach using five-brane webs [15-27]. We will follow the M-theory approach by combining various idea proposed in [13, 28], and [29]. Conceptually the approach presented here is founded in the connection between $6 \mathrm{~d} \mathcal{N}=(1,0)$ and $5 \mathrm{~d} \mathcal{N}=1$ SCFTs by circlecompactification with holonomies in the $6 \mathrm{~d}$ flavor symmetry. Such a dimensional reduction generically results in 5d theories that flow to non-trivial UV fixed points. Alternatively, we can think of the resulting theories as arising from the marginal 5d theory, which is obtained by reducing the $6 \mathrm{~d}$ theory on $S^{1}$ (without holonomies), and subsequently decoupling hypermultiplets transforming in representations of the (classical) flavor symmetry, by turning on a mass deformations, and subsequently sending them to infinity. The mass deformations 
can be interpreted as Coulomb branch parameters for the theory with a weakly gauged classical flavor symmetry. These form part of an extended Coulomb branch $\mathcal{K}\left(\phi^{i}, m_{f}\right)$ of the $5 \mathrm{~d}$ gauge theory, which in addition to the vacuum expectation values, $\phi^{i}$, of the scalars in the vector multiplet also encodes these masses for hypermultiplets $m_{f}$. This connection is depicted on the left-hand side of figure 1 .

It is this extended Coulomb branch, which we would like to map out systematically, and thus determine the distinct strongly coupled UV fixed points. This can be either achieved by analysing the Coulomb branch structure, or using the M-theory realization in terms of singular Calabi-Yau threefolds. We will consider both approaches - see figure 2:

Part I: geometry. In M-theory the extended Coulomb branch is parametrized in terms of the relative extended Kähler cone of the singular Calabi-Yau threefold which underlies the $5 \mathrm{~d}$ marginal theory (and thereby the parent $6 \mathrm{~d} \mathcal{N}=(1,0)$ theory that the marginal theory flows to in the UV). This Kähler cone parametrizes the distinct resolutions of singularities of the Calabi-Yau threefold. The main result in this paper is to find a succinct parametrization of these in terms of what we call combined fiber diagrams (CFDs) [8], which allow us to determine the distinct 5d SCFTs descending from a given $6 \mathrm{~d}$ theory. We illustrate the power of this approach by determining the rank one and rank two classification, ${ }^{1}$ and further we study a large class of examples of 5d SCFTs that are descendants of conformal matter theories. A systematic analysis of the higher rank theories will appear in subsequent work [30].

Part II: box graphs and Coulomb branch phases. In the companion paper [31] we systematically explore the Coulomb branches using the combinatorial device that was called box graphs in [28]. This analysis emphasizes the weakly coupled 5d gauge theory phases that flow to the SCFT, and complements the classification obtained from the geometric approach.

At this point we should clarify the distinctly new aspects of the current proposal towards the classification of 5d SCFTs. We develop a new approach to the geometric classification, which provides a purely combinatorial derivation of all relevant geometries. This approach not only easily extends to higher rank SCFTs, it, at the same time, encodes strongly coupled data such as the (generically enhanced) flavor symmetry for the SCFT, as well as mass deformations, which trigger flows between 5d SCFTs. It is due to these characteristics that we pursue the current approach. Related work, e.g. [6], determines the geometries relevant for the construction of the rank two 5d SCFTs, however the characterization of the geometries does not keep track of the strongly-coupled data in the way that our approach manifestly achieves. When applied to rank two theories, the geometries we find are birationally equivalent to the ones in [6]. However, we choose a description that tracks the flavor symmetries and systematically encodes the relevant deformations at every step of the classification. This seems a natural way to organize the classification of $5 \mathrm{~d}$

\footnotetext{
${ }^{1}$ The rank refers to the dimension of the Coulomb branch, or equivalently, to the rank of the weaklycoupled gauge group, if such a description exists.
} 


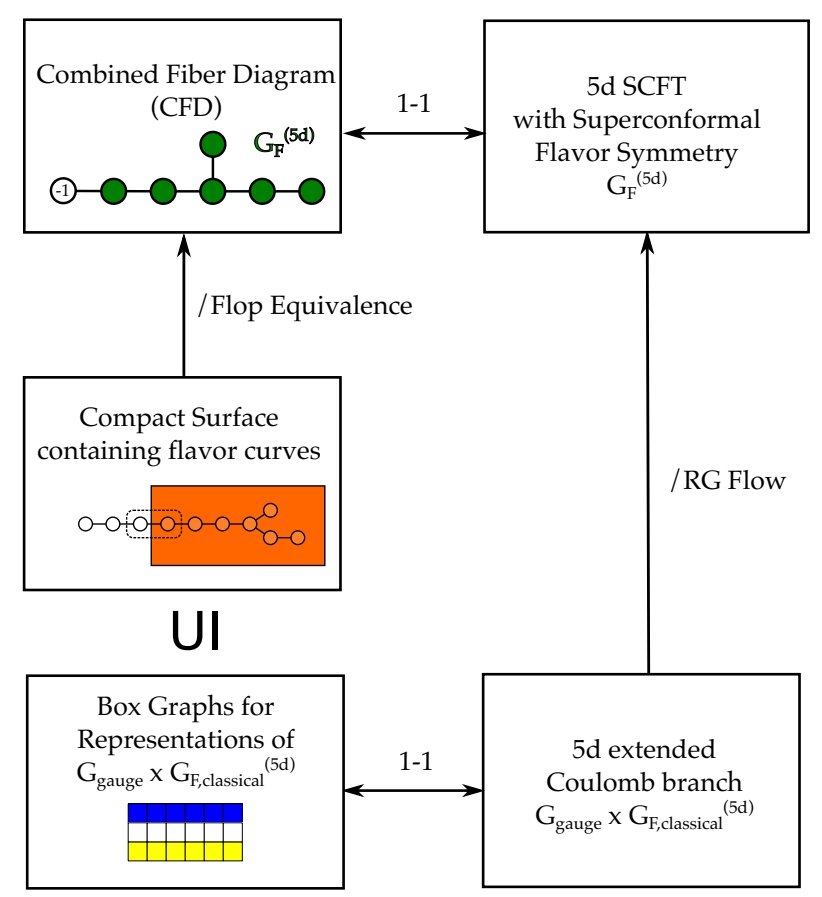

Figure 2. This diagram shows schematically the approaches taken in the present paper and in the companion paper [31], where box graphs and Coulomb branch phases will be discussed. The CFDs were initially introduced in [8] and encode (among other things) the strongly-coupled flavor symmetry $G_{\mathrm{F}}^{(5 d)}$ of the $5 \mathrm{~d}$ SCFT. The present paper provides the geometric foundation for and a derivation of CFDs using the structure of "flavor curves" within compact surfaces in the resolution of Calabi-Yau threefold singularities. In [31] the focus will be on a derivation using weakly-coupled gauge theory descriptions, whenever these exist.

SCFTs, and as we will show, it comes with the added benefit of providing a very efficient algorithm for the classification.

That $5 \mathrm{~d}$ gauge theories often experience a non-trivial flavor symmetry enhancement at their superconformal fixed point was first noted in $[5,25]$. To detect these enhanced symmetries usually requires an analysis of the spectrum of operators charged under the instantonic $\mathrm{U}(1)_{T}$ symmetry, or the computations of protected quantities such as the superconformal index [32-41]. In some cases, the flavor symmetry at strong coupling can be also understood by analyzing string junctions between 7-branes in a $(p, q)$-fivebrane web, see [42] for rank two examples. An alternative approach consists of the study of the Higgs branch at infinite coupling by compactifying the $5 \mathrm{~d}$ theory on a $T^{2}$, which is a $3 \mathrm{~d} \mathcal{N}=4$ theory. The Coulomb branch of the mirror describes the Higgs branch at infinite coupling of the 5d SCFTs $[43,44]$.

Our proposal in turn encodes the flavor enhancement from the get-go: the strongly coupled flavor symmetry is manifest in the geometries and in the graphical description in terms of CFDs, that we propose. This approach is particularly easy to implement for - though not limited to - theories that descend from $6 \mathrm{~d}$ theories, where the geometric realization manifestly encodes the $6 \mathrm{~d}$ superconformal flavor symmetry. Examples are the 
conformal matter theories [45], which have a particularly nice characterization in terms of non-flat resolutions of the (non-compact) elliptically fibered Calabi-Yau threefold. In the context of F-theory, such fibrations have been systematically studied in [46] for Kodaira fibers, with examples in codimension two and three appearing in [13, 29, 47-57]. Unlike the more commonly studied resolutions of minimal collisions of elliptic singularities [58-62], which result in complex one-dimensional fibers, non-minimal singularities require insertions of complex surfaces, $S_{i}$, into the fiber in order to resolve the singularity. Such fibrations, where the fibers are not equidimensional and thus have higher dimensional fiber components, are called non-flat as the projection defining the fibration is not a flat morphism. Similarly we shall refer to a resolution of singularities whose resulting smooth geometry is a non-flat fibration as a non-flat resolution.

We should stress, however, that our approach using CFDs to characterize 5d SCFTs is not limited to geometries that admit a non-flat resolution, but can be used to characterize any crepant resolution of the elliptic Calabi-Yau threefold that underlies a given 6d SCFT. We will encounter examples that are not based on non-flat resolutions in the context of rank two theories.

Throughout, the geometries that we will consider have non-minimal singularities in codimension two, i.e., over points, in the base, $B$, and the singular elliptic Calabi-Yau threefolds $Y$ have a Weierstrass model,

$$
y^{2}=x^{3}+f(u, v) x+g(u, v),
$$

where $(u, v)$ are local coordinates on the base $B$. The non-minimal singularity at $u=$ $0=v$ will correspond to collisions of two non-compact curves in the base, $u=0$ and $v=0$, above which the fiber has standard minimal Kodaira singularities, associated to some Lie algebras $\mathfrak{g}_{\nu}, \nu=u, v$. F-theory compactified on $Y$ gives a $6 \mathrm{~d}$ SCFT, with flavor symmetry $\mathfrak{g}_{\mathrm{F}}^{(6 \mathrm{~d})}[45,63,64]$, and it is this flavor symmetry, and the remnants of this symmetry that percolate down to $5 \mathrm{~d}$, which we will encode in our characterization of the resolution geometries, and in their graphical presentation in terms of CFDs.

There are two approaches to resolve an elliptically fibered Calabi-Yau threefold with non-minimal singularities: one approach, most commonly used in F-theory, is to blow up the non-minimal locus $u=v=0$ in the base successively, until the resulting fibration only has minimal Kodaira singularities. We refer to this, in reference to its interpretation in the field theory, as the tensor branch geometry. This introduces compact surfaces, $S_{i}$, which are ruled over the blow-up curves in the base.

Alternatively, it is useful in certain cases to blow up the fiber of the elliptic threefold $Y$ (without changing $B$ ). This approach is particularly useful for $5 \mathrm{~d}$ theories that descend from $6 \mathrm{~d}$ conformal matter theories, where the $6 \mathrm{~d}$ flavor symmetry can be realized such that $\mathfrak{g}_{\mathrm{F}}^{(6 \mathrm{~d})}=\mathfrak{g}_{u} \oplus \mathfrak{g}_{v}$. The resolution of the codimension one Kodaira singular fibers gives rise to non-compact ruled surfaces, the so-called Cartan divisors,

$$
\mathbb{P}_{l}^{1} \hookrightarrow D_{l}^{\mathfrak{g}_{\nu}} \rightarrow\{\nu=0\}
$$

which are fibered over the codimension one loci $\nu=0$ (associated to the simple roots of $\mathfrak{g}_{\nu}$ ). Over codimension two, these rational curves can become reducible, in addition however, 
due to the non-minimal singularity, the fiber resolution also introduces compact surfaces $S_{i}$ over the codimension two locus $u=0=v$. The resulting smooth model is therefore not fibered by complex one dimensional curves, but includes higher dimensional surface components - the hallmark of a non-flat fibration.

The two approaches are, of course, birationally equivalent. We argue that whichever way one chooses to resolve the singularity, it will be key to retain the information about the intersection between the compact surfaces $S_{j}$ (either from base blow-ups or non-flat resolutions) and the non-compact Cartan divisors (1.2), in order to manifestly encode the flavor symmetries of the $5 \mathrm{~d}$ SCFTs.

In gauge theoretic terms, a resolved geometry corresponds to a point in the extended Coulomb branch of the $5 \mathrm{~d}$ marginal theory. The compact surface components, $S_{i}$, that resolve the non-minimal singularity realize the gauge group of the effective field theory, while the non-compact divisors $D_{l}^{\mathfrak{g}_{\nu}}$ furnish the flavor symmetry. The flavor symmetry is determined by fibral $\mathbb{P}^{1} \mathrm{~s}$, that are contained within the surface components $S_{i}$ - we will refer to these curves as flavor curves. In the singular limit, which corresponds to taking the volume of all $S_{i}$ to zero, and thus the gauge coupling to infinite, the flavor curves also collapse to zero volume, and contribute to the flavor symmetry at the strongly coupled point. The flavor symmetries we find in this way are in agreement with all known flavor enhancements in 5d SCFTs at rank one and two and predict new flavor symmetries at higher rank.

The CFDs encode essentially the generators of the Mori cone of the compact (reducible) surfaces. From these we can furthermore determine a set of BPS states, which arise from wrapped M2-branes over complex curves. These become massless particles in the SCFT limit. For genus 0 curves, their spectrum can be straightforwardly computed in our constructions, as we will demonstrate for spin 0 and spin 1 states in all rank one and rank two theories we construct.

In the present paper, we will have less of a focus on the weakly coupled gauge theory interpretations of the geometries. It should be stressed, however, that the geometries equally contain the gauge theory data [5]. Using the concept of box graphs introduced in [28], we systematically map out the Coulomb branch phases in the companion paper [31]. This agrees with the present geometric analysis, whenever a weakly coupled gauge theory description is available.

\section{$1.1 \quad$ Strategy}

We now summarize our strategy. The starting point is a 6d SCFT from which we determine upon circle reduction the $5 \mathrm{~d}$ marginal theory, to which we associate three quantities: a resolved Calabi-Yau threefold, an effective gauge theory description and a graph, called a CFD. From this data, we determine all 5d descendant SCFTs, which can be obtained from the marginal theory by relevant deformation and RG-flow, as well as key physical features, by applying a straightforward algorithm.

We support our proposal using a geometric and a gauge theoretic approach, respectively. 
Part I: geometry. A given marginal theory is associated to a crepant resolution of an elliptic Calabi-Yau threefold with a non-minimal singularity. From such a smooth geometry, one can extract an object we refer to as a marginal combined fiber diagram, which allows us to track all descendant SCFTs in terms of so-called descendant CFDs. Each descendant CFD similarly is associated to a different crepant resolution of a Calabi-Yau threefold. Each CFD corresponds to a 5d SCFT, and encodes the superconformal flavor symmetry as well as the relevant deformations that trigger flows to another SCFTs.

The CFD is a graph with vertices that are curves inside the reducible surface $\mathcal{S}=\bigcup_{k} S_{k}$ with self-intersection (inside $\mathcal{S}$ ) of -2 or higher. The $(-2)$-curves correspond to the $\mathbb{P}^{1}-$ fibers of $D_{l}^{\mathfrak{g}_{\nu}}$ and give rise to the non-abelian part of the flavor symmetry of the SCFT - the above-mentioned flavor curves. Vertices that correspond to $(-1)$-curves can be removed, which corresponds to flop transitions that map the curve out of $\mathcal{S}$; they correspond physically to the hypermultiplets that can decouple via mass deformations. Such CFDtransitions allow charting the entire tree of descendant SCFTs systematically. Finally, the CFDs contains higher self-intersection curves which are related to abelian flavor factors and play a key role in determining the BPS states.

To obtain a comprehensive description it is thus key to determine the CFD associated to the marginal theory, from which all descendants are obtained by CFD-transitions. For example, the unique rank one marginal theory originating from an $S^{1}$-compactification of the 6d rank one E-string has the CFD

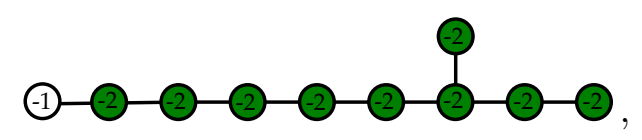

where the $(-2)$-curves are the colored vertices, and correspond precisely to the flavor curves. This geometry is obtained by studying the curves in the compact surface, which is a generalized del Pezzo surface, $\mathrm{gdP}_{9}$. This is a rational elliptic surface and the $(-1)$ curve is the zero-section, i.e., the copy of the base $\mathbb{P}^{1}$ in the surface; the (-2)-curves intersecting in the affine $\mathrm{E}_{8}$ Dynkin diagram correspond to the geometric realization of the flavor symmetry of the $5 \mathrm{~d}$ marginal theory inherited from the E-string.

For higher rank $5 \mathrm{~d}$ theories, the corresponding CFDs should be thought of as an equivalence class of geometries, related to each other by flops which do not correspond to mass deformations, but simply to different gauge theory descriptions that yield the same UV fixed point. In this way the CFDs are more effective in their characterization of the SCFTs than any given resolution of a singular geometry.

Part II: Coulomb branch and box graphs. Another path to support out CFDapproach is to consider the effective gauge theory description. To a marginal theory one can also associate in general multiple gauge theoretic or quiver descriptions ${ }^{2}$ To each gauge or quiver description, we can again encode the extended Coulomb branch diagrammatically, in terms of so-called box graphs [28, 65-67], which were used to characterize crepant resolutions of minimal singularities in elliptic fibrations. These are representation-theoretic ways of

\footnotetext{
${ }^{2}$ Geometrically, these correspond to rulings of the surface components $S_{k}$.
} 
characterizing the different Coulomb branch phases, and in the present application, encode in particular the mass deformations that trigger flows to other SCFTs.

To exemplify this, consider again the rank one marginal theory in 5 d, i.e. the circlereduction of the rank one E-string theory. This admits an $\mathrm{SU}(2)_{\mathrm{G}}$ gauge theory description with eight fundamental flavor hypermultiplets, transforming under a classical flavor symmetry, $\mathrm{SO}(16)_{\mathrm{F}}$. The matter fields in the $(\mathbf{2}, \mathbf{1 6})$ representation of $\mathrm{SU}(2) \times \mathrm{SO}(16)$ can be represented in terms of a representation graph, that encodes whether the corresponding weight or its negative is in the Coulomb branch (indicated by blue/yellow coloring); this comprises the box graph for this gauge theory description

$(2,16):$

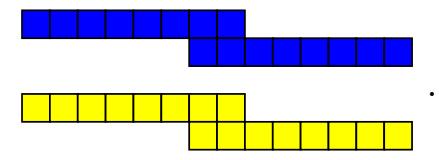

Here, each diagram is a $\mathbf{1 6}$ of $\mathrm{SO}(16)$, and the simple root of $\mathrm{SU}(2)$ maps between the two diagrams. In the companion paper [31] we show how this characterization of the gauge theory phases encodes the mass deformations and analogous box graph descriptions for all descendant $5 \mathrm{~d}$ theories. We also determine how the classical flavor symmetry is enhanced to the superconformal one. In summary, we will show in [31] that for 5d SCFTs, which admit a weakly-coupled gauge theory description, the box graphs encode equivalent information to the geometric approach of the present paper. Furthermore, as in the geometric case, equivalence classes of box graphs, which are inequivalent gauge theory phases that yield the same UV fixed point, can be packaged together into a CFD. In this way we also able to provide an independent derivation of the tree of SCFTs/CFDs, using box graphs and transitions between box graphs.

The present paper is organized as follows: section 2 contains background on $5 \mathrm{~d}$ gauge theories, SCFTs and their realization in M-theory, as well as resolutions of singular elliptic fibrations - all central to our subsequent endeavors. These ideas are combined in section 3 : we begin in section 3.1 by detailing the dictionary between M-theory on resolutions of elliptic threefolds and 5d SCFTs. The proposal is exemplified by determining all rank one geometries by considering non-flat resolutions of the elliptic fibration in section 3.2. In sections 3.3 and 3.4, we determine the resolutions for the marginal theories (which are the essential starting points for our subsequent analysis of the descendant theories) and some examples of explicit resolutions for rank two and higher. In section 4 we propose a succinct way of encoding salient properties of the crepant resolutions into graphs, the combined fiber diagrams (CFDs). We define CFDs associated to crepant resolutions, and then demonstrate the result by determining all rank one theories and rank two theories, using this proposal. Furthermore, we determine the marginal theories for the $\left(\mathrm{E}_{n}, \mathrm{E}_{n}\right)$ and $\left(\mathrm{E}_{8}, \mathrm{SU}(n)\right)$ conformal matter theories and determine their descendants. In section 5 we discuss the BPS states that are encoded in the CFDs and compute them for rank one and rank two theories. We conclude in section 6. There are various appendices containing summaries and details of our analysis. Most noteworthy are the summary tables for the rank two theories in appendix A, specifically appendix A.2, which summarizes for each rank one and two theory the corresponding CFDs, the strongly-coupled flavor symmetry, the 
weakly-coupled gauge theory description (if it exists), and the spin 0 and spin 1 BPS states. The RG-flow trees, which are determined by considering the CFDs and their transitions, starting with the marginal CFDs are shown in figure 10 for rank one, and in figures 11, 12, and 13 for rank two.

\section{M-theory, 5d SCFTs, and resolutions of elliptic fibrations}

Due to their intrinsically strongly-coupled nature, 5d SCFTs have been traditionally studied via their low-energy effective gauge theory phase. As we will review now, the Coulomb branch structure of this infrared (IR) description motivates the approach of geometric engineering in M-theory. Moreover, we discuss - in the framework of M-/F-theory duality - how the geometry can also capture essential physical features of theories that descend from circle reductions of $6 \mathrm{~d} \mathcal{N}=(1,0)$ SCFTs.

\section{$2.15 d$ SCFTs, gauge theories, and Coulomb branches}

Gauge theories in 5d can be viewed as effective low-energy descriptions of an SCFT in the $\mathrm{UV}$, which is deformed by the following relevant operator

$$
\int d^{5} x g_{\mathcal{O}} \mathcal{O}(x) \sim \frac{1}{g_{\mathrm{YM}}^{2}} \int d^{5} x \operatorname{Tr}\left(F^{\mu \nu} F_{\mu \nu}\right) .
$$

The explicit form of this operator is itself part of the low-energy effective description, as $g_{\mathrm{YM}}$ has negative scaling dimension, which means that the theory is non-renormalizable. In other words the gauge theory description is not consistent at all scales. From the gauge kinetic term we infer the potential existence of an RG-fixed point at $g_{\mathrm{YM}} \rightarrow \infty$, which is a strongly coupled UV SCFT. Moreover, there can be several operators like (2.1), leading to different IR gauge theories, which are dual in the UV.

A $5 \mathrm{~d} \mathcal{N}=1$ gauge theory with gauge group $G_{\text {gauge }}$ and associated gauge Lie algebra $\mathfrak{g}$ has the following multiplets:

$$
\begin{aligned}
\text { Vector multiplet in } \mathbf{A d}(\mathfrak{g}): & \mathcal{A}=\left(A_{\mu}, \phi^{i}, \lambda\right), \\
\text { Hypermultiplets in } \mathbf{R}_{\mathfrak{g}}: & \mathbf{h}=\left(h \oplus h^{c}, \psi\right) .
\end{aligned}
$$

The hypermultiplets transform in representations of the classical flavor symmetry $G_{\mathrm{F}, \mathrm{cl}}$ of the effective gauge theory. Moreover, any $5 \mathrm{~d}$ gauge theory has instanton operators which are charged under an abelian global symmetry $\mathrm{U}(1)_{T}$ associated to the current

$$
J_{T}=\frac{1}{8 \pi^{2}} \star \operatorname{Tr}(F \wedge F) .
$$

If $G_{\text {gauge }}$ is semi-simple, each simple gauge factor has a topological $\mathrm{U}(1)_{T}^{(n)}$. Nonperturbative effects can lead to an enhanced superconformal flavor symmetry, which we denote by

$$
G_{\mathrm{F}} \supset G_{\mathrm{F}, \mathrm{cl}} \times \prod_{n=1}^{N} \mathrm{U}(1)_{T}^{(n)},
$$

with $\operatorname{rank}\left(G_{\mathrm{F}}\right)=\operatorname{rank}\left(G_{\mathrm{F}, \mathrm{cl}}\right)+N$. 
The Coulomb branch of a $5 \mathrm{~d}$ gauge theory is parametrized by the vacuum expectation values (vevs), $\left\langle\phi^{i}\right\rangle$, of the real scalars in the vector multiplets. These generically break the gauge group $G_{\text {gauge }}$ to its Cartan $\mathrm{U}(1)^{r}$. The effective Lagrangian that governs the dynamics on the Coulomb branch is

$$
\mathcal{L}_{\text {eff }}=G_{i j} d \phi^{i} \wedge \star d \phi^{j}+G_{i j} F^{i} \wedge \star F^{j}+\frac{c_{i j \ell}}{24 \pi^{2}} A^{i} \wedge F^{j} \wedge F^{\ell}+\ldots,
$$

where the couplings are determined by derivatives of the prepotential $\mathcal{F}$, which is a real cubic function of the vevs of $\phi^{i}$

$$
\begin{aligned}
G_{i j} & =\frac{\partial^{2} \mathcal{F}}{\partial \phi^{i} \partial \phi^{j}}, \\
c_{i j \ell} & =\frac{\partial^{3} \mathcal{F}}{\partial \phi^{i} \partial \phi^{j} \partial \phi^{\ell}} .
\end{aligned}
$$

Since the Chern-Simons term is not gauge invariant, its coefficient must be integer, $c_{i j \ell} \in \mathbb{Z}$ to avoid axial anomalies [5, 68]. The prepotential has a classical and one-loop contribution

$$
\begin{aligned}
\mathcal{F} & =\mathcal{F}_{\text {classical }}+\mathcal{F}_{1 \text {-loop }} \\
& =\left(\frac{1}{2 g_{\mathrm{YM}}^{2}} C_{i j} \phi^{i} \phi^{j}+\frac{k}{6} d_{i j \ell} \phi^{i} \phi^{j} \phi^{\ell}\right)+\frac{1}{12}\left(\sum_{\alpha \in \Phi_{\mathfrak{g}}}\left|\alpha_{i} \phi^{i}\right|^{3}-\sum_{\mathbf{R}_{f}} \sum_{\lambda \in \mathbf{W}_{\mathbf{R}_{f}}}\left|\lambda_{i} \phi^{i}+m_{f}\right|^{3}\right),
\end{aligned}
$$

where $d_{i j \ell}=\frac{1}{2} \operatorname{tr}_{\text {fund }}\left(T_{i}\left(T_{j} T_{\ell}+T_{\ell} T_{j}\right)\right), C_{i j}$ is the Cartan matrix of $\mathfrak{g}$, and $k$ the halfinteger quantized classical Chern-Simons level. The roots of $\mathfrak{g}$ are $\Phi_{\mathfrak{g}}$, and weights of the representation $\mathbf{R}_{f}$ by $\mathbf{W}_{\mathbf{R}_{f}}$. Finally, $m_{f}$ are the masses of the hypermultiplets.

The prepotential determines different phases of the gauge theories as well as the existence of a UV fixed point. At $g_{\mathrm{YM}} \rightarrow \infty$ the effective Lagrangian description breaks down due to the appearance of infinitely many light states. The phases of the gauge theory form the extended Coulomb branch

$$
\mathcal{K}\left(\phi^{i}, m_{f}\right)
$$

which includes the vevs of scalars $\phi^{i}$ but also the masses of hypermultiplets. This can be thought of as the Coulomb branch of the theory, where we in addition weakly gauge the flavor symmetry $G_{\mathrm{F}, \mathrm{cl}}$. These mass parameters can be interpreted as Coulomb branch parameters, where we have weakly gauged the classical flavor symmetry $G_{\mathrm{F}, \mathrm{cl}}$.

Our approach in this paper is based on the observation that these gauge theory structures are equivalently realized in terms of M-theory compactifications on Calabi-Yau threefolds [5, 9, 69-72]. In particular, the non-abelian gauge degrees of freedom arise from M2branes wrapping collapsed holomorphic curves at singularities. In this correspondence, the Coulomb branch (2.8) is identified with the so-called extended Kähler cone associated to a singular Calabi-Yau threefold. This cone is defined by the union of all the Kähler cones associated to the possible crepant resolutions of the singularity. Each of these resolutions is geometrically given by a collection $\mathcal{S}=\bigcup_{j} S_{j}$ of compact surfaces $S_{j}$, where the surfaces are in one-to-one correspondence with the Cartans of the vector multiplets and with the 
Coulomb branch scalars. On the other hand, each mass parameter $m_{f}$ is associated with a non-compact surface $D_{f}$, and for fixed choices of $m_{f}$ there are subcones

$$
\left.K\left(\phi^{i}\right)\right|_{\text {fixed } m_{f}} \subset \mathcal{K}\left(\phi^{i}, m_{f}\right) .
$$

The origins of these subcones define different singular limits in which the compact surfaces $S_{j}$ collapse to zero volume, which in the gauge theory correspond to distinct $5 \mathrm{~d}$ UV fixed points.

Before we discuss in detail the dictionary between Calabi-Yau geometry and 5 d physics, we point out that the extended Coulomb branch $K\left(\phi^{i}, m_{f}\right)$ has an elegant and systematic description in terms of so-called box graphs [28], which encode the extended Coulomb branch in terms of representation-theoretic data of

$$
G_{\text {gauge }} \times G_{\mathrm{F}, \mathrm{cl}}
$$

We will explore this gauge-theoretic approach in the companion paper [31].

\subsection{Geometric engineering of 5d gauge theories and SCFTs}

The gauge theoretic content of the last section has a counterpart in the realization of $5 \mathrm{~d}$ SCFTs in M-theory on singular Calabi-Yau threefolds [5]. Associated to a singular CalabiYau $Y$ is a resolution that retains the Calabi-Yau condition, a so-called crepant resolution. The process of resolving the singularity introduces compact divisors, i.e., complex surfaces, $S_{i}$, into the geometry. The space of crepant resolutions of the singularity should be thought of as playing the role of the extended Coulomb branch of the gauge theory. The precise dictionary is as follows:

- Cartan subgroup of the gauge group: a compact divisor $S$ is dual to a $(1,1)$-form $\omega^{(1,1)}$, which in turn can be used to dimensionally reduce the M-theory three-form $C_{3}=\omega^{(1,1)} \wedge A$, where $A$ is a $\mathrm{U}(1)$-gauge field. The gauge coupling is set by the volume of $S$. The number of compact surface components $S_{i}, i=1, \cdots, r$, sets the rank of the weakly-coupled gauge theory.

- Non-abelian gauge symmetry: the enhancement to a non-abelian gauge group results from rulings of the compact surface components. A surface $S$ is ruled, if it is fibered by rational curves $f$ over a curve $\Sigma$,

$$
f \hookrightarrow S \rightarrow \Sigma,
$$

with the intersection numbers

$$
S \cdot f=-2, \quad f \cdot S f=0 .
$$

This allows collapsing the surface along the fibers $f$ to curve $\Sigma$ worth of singularities, inside of the Calabi-Yau threefold. M2-branes wrapped on $f$ become massless in this limit and furnish the $\mathrm{W}$-bosons for the enhancement to a non-abelian gauge symmetry. It is possible for $r$ surfaces $S_{i}$ to intersect pairwise along curves $\Sigma_{i j}=$ 
$S_{i} \cap S_{j}$ that are (multi-)sections of the rulings $f_{i} \hookrightarrow S_{i} \rightarrow \Sigma_{i}$ on both surfaces. In this case, collapsing all $f_{i}$ leads to a simple rank $r$ non-abelian gauge group $G_{\text {gauge }}$ determined by the Cartan matrix

$$
C_{i j}^{G_{\text {gauge }}}=-S_{i} \cdot f_{j}
$$

The genus $g\left(\Sigma_{i j}\right)$ of the intersection curve is computed by

$$
S_{j} S_{j+1}^{2}+S_{j+1} S_{j}^{2}=2 g\left(\Sigma_{i j}\right)-2 .
$$

The fibers that rule the configuration of surfaces $\mathcal{S}=\bigcup_{j} S_{j}$ will be denoted by

$$
\{f\} \equiv \bigcup_{j} f_{j} \hookrightarrow \mathcal{S}
$$

- Matter: if a ruling $f_{i} \hookrightarrow S_{i}$ has reducible fibers, such that the rational curves splits (i.e., $f_{i}=\sum_{l} C_{i}^{(l)}$ in homology), M2-branes wrapping $C_{i}^{(l)}$ give rise to charged matter states which become massless in the singular limit when $S_{i}$ collapses to $\Sigma_{i}$.

- Quiver theories: a weakly-coupled description of an SCFT can also be in terms of a quiver gauge theory. For instance a quiver with gauge groups $G_{1} \times G_{2}$ is realized, if there are surfaces $S_{G_{i}}, i=1,2$ (contributing to the gauge groups $G_{i}$ in the above fashion), intersecting each other along $\Sigma$, which is a special fiber of the ruling on both surfaces $S_{G_{i}}$. In this case, collapsing all fibers of these rulings leads to massless states from M2-branes on $\Sigma$, which are charged as bifundamentals of $G_{1} \times G_{2}$.

- Prepotential: from the M-theory compactification is a polynomial in the Kähler parameters $\phi^{i}$ dual to the divisors $S_{i}$ we can define the geometric prepotential

$$
\mathcal{F}_{\text {geo }}=\frac{1}{6} c_{i j \ell} \phi^{i} \phi^{j} \phi^{\ell} \quad \text { with } \quad c_{i j \ell}=S_{i} \cdot S_{j} \cdot S_{\ell} .
$$

This is to be identified with the cubic part of the field theoretic prepotential (2.7), which receives classical and one-loop contributions

$$
c_{i j \ell}=S_{i} \cdot S_{j} \cdot S_{\ell} \stackrel{!}{=} k d_{i j \ell}+c_{\text {one-loop }, i j \ell} .
$$

In practice, the prepotential matching allows us to compute the classical ChernSimons level $k$ from geometry, see appendix B for the rank two theories.

- Dualities: a surface can have multiple rulings, which give rise to dual gauge theory descriptions of the same SCFT. For example, in the rank two theories, two surfaces $S_{1}$ and $S_{2}$ intersecting along $\Sigma$ can have three distinct types of rulings $f_{i}^{(n)} \hookrightarrow S_{i}$, $n=1,2,3$, corresponding to the following weakly-coupled gauge theory descriptions:

1. $\Sigma$ is a section for both surfaces, i.e., $\Sigma \cdot S_{1} f_{1}^{(1)}=\Sigma \cdot S_{2} f_{2}^{(1)}=1$. This gives rise to an $\mathrm{SU}(3)$ theory. 
2. $\Sigma$ is a section for $S_{1}$ and a bi-section for $S_{2}$, i.e., $2 \Sigma \cdot S_{1} f_{1}^{(2)}=\Sigma \cdot S_{2} f_{2}^{(2)}=2$. This realizes an $\operatorname{Sp}(2)$ theory.

3. $\Sigma$ is a special fiber in both surfaces, i.e., $\Sigma \cdot S_{i} f_{i}^{(3)}=0$. This correspond to an $\mathrm{SU}(2) \times \mathrm{SU}(2)$ quiver description.

- Coulomb branch phases: in general, there can be different configurations of surfaces $S_{k}$ that give rise to the same gauge theory upon flop transitions along suitable rulings within the reducible surface $\mathcal{S}$. This is the geometric incarnation of different chambers within the Coulomb branch of a $5 \mathrm{~d} \mathcal{N}=1$ gauge theory. As one crosses the interfaces between two such chambers, the masses of certain states undergoes a sign change. This is distinct from flopping curves in or out of $\mathcal{S}$, which in contrast gives rise to different SCFTs.

- Relevant deformations and flows to new SCFTs: while flop transitions that map curves out of $\mathcal{S}$ correspond to mass deformations in the gauge theory, physically, it involves sending one of the flavor masses, $m_{f}$, to $\pm \infty$ and effectively decouples the corresponding hypermultiplet. Geometrically, this is due to the curve being flopped out of $\mathcal{S}$, and thereby not getting collapsed in the singular limit (i.e., the associated state remains massive). This decoupling process yields an effective theory with a different UV fixed point. In case the effective description has an appropriate gauge theory interpretation, the classical Chern-Simons level shifts as

$$
k \rightarrow k+\operatorname{sign}\left(m_{f}\right) \frac{1}{2}
$$

- Absence of weakly-coupled gauge theory description: the geometric description is slightly more general as it captures SCFTs that do not allow a weakly-coupled description. This occurs e.g. in rank one for the " $E_{0}$ theory" [9], with the number of such theories increasing in higher rank. Geometrically, this occurs, when two surfaces $S_{1,2}$ only have rulings such that $\Sigma=S_{1} \cap S_{2}$ is a fiber of one ruling, but a (multi)section of the other. There is no "classical" non-abelian gauge theory phase, because collapsing either surface along its rulings results in light non-perturbative states from the forced collapse of the other surface. Such geometries are nevertheless consistent M-theory compactifications and give rise to non-trivial strongly interacting limits, corresponding to 5d SCFTs.

Importantly, the geometric description of mass deformations puts theories without effective gauge descriptions on the same footing as theories with gauge theory descriptions. While certain states that can be decoupled may be non-perturbative states from a gauge theoretic perspective, the geometry uniformly characterizes these as curves that can be flopped out of the reducible surface $\mathcal{S}$. Such curves play a vital role in determining all descendant SCFTs obtainable from a given SCFT via mass deformations. 


\subsection{SCFTs and flavor from geometry}

The SCFT limit of a gauge theory, where $\phi^{i} \rightarrow 0$ with fixed $m_{f}$, corresponds in geometry to collapsing the reducible compact surface $\mathcal{S}=\bigcup_{j} S_{j}$ to a point. In this limit, nonperturbative states from M2-branes wrapping (multi-)sections of the rulings on $S_{j}$ become part of the spectrum, signaling a breakdown of the effective description.

The existence of such a strongly coupled UV fixed point poses certain convexity constraints on the prepotential (2.7) as a function on the extended Coulomb branch. Translated into geometry, this implies conditions on the singular limits of surface configuration $\mathcal{S}$ [5]. Essentially, the condition is that the singularity one obtains from the collapse is not too severe, and allows for a crepant resolution. Such a singularity is called a canonical singularity. Importantly, these conditions are purely geometric and thus also apply to cases without an effective gauge theory description.

Moreover, the geometric perspective easily accommodates the feature of UV dualities, as a canonical singularity can have different resolutions corresponding to different effective gauge descriptions of the same SCFT. This is reflected by certain details, e.g., the intersection curves between different surfaces, being vital to the gauge theory description, but not to the SCFT. In contrast, the contractible curves inducing mass deformations have to be kept track across different, dual phases.

A key feature of the UV fixed points is that the flavor symmetry of the theory can enhance compared to the effective description as a gauge theory. While the classical flavor symmetry $G_{\mathrm{F}, \mathrm{cl}}$ of an effective gauge theory is easily inferred from the spectrum of hypermultiplets, the superconformal flavor group $G_{\mathrm{F}}$ is oftentimes difficult to determine, as it is an intrinsically strongly-coupled datum. In cases when the SCFT has a gauge theory phase in the IR, the intricate enhancement (2.4) can be computed via the superconformal index [32]. However, such methods fail when no effective description is available.

A central point that we will make in this paper is that the geometry does in fact track the flavor symmetries via the collapse of non-compact divisors intersecting the compact surfaces $\mathcal{S}[10,13]$. Concretely, if a non-compact divisor $D \subset Y$ is ruled, $F \hookrightarrow D \rightarrow W$, then collapsing $F$ generates orbifold singularities fibered along a non-compact curve isomorphic to $W$. More generally, if the generic fibers of multiple non-compact divisors intersect in a Dynkin diagram, then the above collapse produces a curve of the corresponding ADE singularities.

In particular, when $\mathcal{S}$ collapses along the ruling $\{f\}$ (2.15), a subset of generic fibers $F$ contained in $\mathcal{S}$ may be forced to collapse to a curve, leading to singularities of ADE-type - this determines the classical flavor symmetry $G_{\mathrm{F}, \mathrm{cl}}$. When furthermore collapsing $\mathcal{S}$ to a point, this flavor symmetry can enhance to $G_{\mathrm{F}}$, which generically is larger than $G_{\mathrm{F}, \mathrm{cl}}$.

The classical flavor symmetry $G_{\mathrm{F}, \mathrm{cl}}$ depends on the choice of ruling $\{f\} \hookrightarrow \mathcal{S}$, and may only be manifest in certain geometric phases. However, the superconformal flavor symmetry $G_{\mathrm{F}}$ only depends on the curves in $\mathcal{S}$ that collapse in the singular limit. We will refer to these as flavor curves. A particularly nice way of extracting these flavor curves arises for $5 \mathrm{~d}$ theories that descend from $6 \mathrm{~d}$ conformal matter theories, where the $6 \mathrm{~d}$ flavor symmetry is manifest in the elliptic fibration. By considering non-flat resolutions of such 
singularities, the flavor curves have a very simple presentation and are manifest in the resolved geometries.

\section{$2.45 d$ marginal theories}

A special class of $5 \mathrm{~d}$ gauge theories that arise by circle-reduction of $6 \mathrm{~d}$ SCFTs are so-called marginal theories. These are effective theories whose UV-completion is not an honest $5 \mathrm{~d}$ SCFT, but rather the $6 \mathrm{~d}$ SCFT itself. This limiting theory, dubbed 5d Kaluza-Klein (KK) theory in [6], is the M-theory compactification on the fully singular elliptic fibration, i.e., the geometric limit where all compact $\left(S_{j}\right)$ and non-compact $\left(D_{i}^{(\nu)}\right)$ exceptional divisors are collapsed to points and curves, respectively.

The marginal theories play in important role in our classification program as they contain the relevant information about the $5 \mathrm{~d}$ SCFTs that descend from a given $6 \mathrm{~d}$ SCFT. Geometrically, the marginal theory can be characterized most straightforwardly by blowing up the base to remove the non-minimal point $u=v=0$, possibly multiple times, until all fiber singularities are of minimal Kodaira type. In $6 \mathrm{~d}$, these blow-ups correspond to giving the scalars of $\mathcal{N}=(1,0)$ tensor multiplets a vev, thus moving the 6d SCFT onto its tensor branch. A subsequent fiber resolution yields a smooth Calabi-Yau threefold with a flat fibration. Note that this resolution process also introduces compact surfaces $S_{j}$, which are now ruled (or elliptically fibered) over the compact rational curves that were introduced into the base to blow up $u=v=0$.

In this smooth space, one can in principle read off all necessary physical data such as the effective gauge descriptions and their mass deformations. There are ongoing efforts, for example in $[73,74]$, towards classifying the smooth geometries associated with marginal theories arising from 6d SCFTs classified by F-theory.

A key feature of the marginal geometry is that the compact surfaces $\mathcal{S}=\bigcup_{j} S_{j}$ contain all codimension one fibers $F_{i}^{(\nu)}$, i.e., the maximal set of flavor curves. This means that when $\mathcal{S}$ is collapsed to a point, the flavor symmetry from codimension one singularities, as discussed in section 2.3, would formally be of affine type. The "affinization" is due to the presence of the Kaluza-Klein U(1) arising in the circle reduction, and is the indicator that the UV physics corresponding to this singular geometry is only appropriately described as a $6 \mathrm{~d}$ theory.

Once one knows the $5 \mathrm{~d}$ marginal theory and its associated "marginal" geometry, mass deformations corresponding to flops generate all descendant theories that have a genuine 5d SCFT limit. Geometrically, these flops move flavor curves $F_{i}$ out of $\mathcal{S}$, such that when we collapse the latter, not all codimension one divisors $D_{i}$ are forced to collapse to curves.

The geometries associated to the $5 \mathrm{~d}$ marginal theories that come from $6 \mathrm{~d}\left(G_{1}, G_{2}\right)$ conformal matter theories can be particularly elegantly characterized in terms of non-flat resolutions of elliptic fibrations. In this case, the flavor curves that are the fibers of the Cartan divisors associated to the two flavor groups $G_{1}$ and $G_{2}$ in $6 \mathrm{~d}$, can be straightforwardly identified inside the non-flat fibers. This is based on the idea of [13], where non-flat fibrations were used in this context. Here, we will construct the marginal geometries for conformal matter theories from non-flat resolutions, where the non-flat fiber is a reducible 
surface $\mathcal{S}$. An advantage of this approach is that $\mathcal{S}$ is by construction contractible, as it arises from crepant resolutions of a singularity in a Calabi-Yau threefold. Thus, the singular limit of blowing down $\mathcal{S}$ gives rise to a well-defined 5d SCFT in M-theory.

\subsection{Singular elliptic Calabi-Yau threefolds}

Given our motivations, we focus on singular Calabi-Yau threefolds that are elliptic fibrations over a non-compact base two-fold $B$. In the context of F-theory [75], these manifolds define $6 \mathrm{~d}$ theories. We assume first of all that the base is smooth. ${ }^{3}$ In complex codimension one in the base, singularities in the fiber are of so-called minimal type, and are classified by the classical results of Kodaira and Néron, and give rise to gauge fields in $6 \mathrm{~d}$. In codimension two, minimal singularities give rise to $6 \mathrm{~d}$ bifundamental matter in hypermultiplets [76-78]. The case of interest in this paper is, however, when the fibration has a codimension two non-minimal singularity. These are indicators of strongly coupled sectors in F-theory, and form the geometric foundation of the recent classification of $6 \mathrm{~d} \mathcal{N}=(1,0)$ SCFTs [2-4]. In $6 \mathrm{~d}$ the canonical way to study these singularities is by blowing up the base, i.e., removing the codimension two non-minimal locus by inserting rational curves, until the singularities all become minimal.

In M-theory on such Calabi-Yau threefolds, there is an alternative approach, which allows resolutions of the singular fiber, keeping the base unchanged. Fiber-resolutions of non-minimal singularities have the key feature that they insert higher-dimensional fiber components. For codimension two non-minimal singularities in Calabi-Yau threefolds these are complex surface components. In this section we summarize the background of how to study such non-flat resolutions.

Consider an elliptically fibered Calabi-Yau threefold $Y$, defined over a complex base surface $B$,

$$
\mathbb{E} \hookrightarrow Y \stackrel{\pi}{\longrightarrow} B,
$$

with projection $\pi$ and generic fiber given by an elliptic curve $\mathbb{E}$. We will assume that this has a section, i.e., a map from the base to the fiber, and thus a Weierstrass model

$$
y^{2}=x^{3}+f x+g
$$

We will generally work with the Tate model of the elliptic fibration,

$$
y^{2}+b_{1} x y+b_{3} y=x^{3}+b_{2} x^{2}+b_{4} x+b_{6},
$$

which is (largely) equivalent to the Weierstrass model, but comes with some added computational benefits $[79,80]$.

The $b_{i}$ are sections of line bundles over the base $B$, i.e., they depend on the base coordinates. The generic fiber above a point in the base will be smooth, and singularities in codimension one occur whenever the discriminant $\Delta=4 f^{3}+27 g^{2}$ vanishes. Let $(u, v)$ denote local coordinates in the base. In general, the discriminant locus $\{\Delta=0\}$ can have multiple components. For our purposes, it will suffice to consider two codimension one

\footnotetext{
${ }^{3}$ We will discuss models where the base has codimension two singularities momentarily.
} 
irreducible components of $\{\Delta=0\}$, which locally are described by $u=0$ and $v=0$. The singularity type in the fiber is determined by the vanishing orders of $b_{i}$ as functions of $u$ and $v$. We will use the notation for resolutions of singularities as introduced in $[28,46]$.

\subsubsection{Codimension one: Kodaira fibers}

Denote by $W_{u}=\{u=0\}$ an irreducible component of the discriminant locus. To each set of vanishing orders in codimension one $\operatorname{ord}_{u}\left(b_{i}\right)$ there is an associated Kodaira singular fiber. These are determined by resolving the singularity, which amounts to introducing new (exceptional) rational curves (i.e., $\mathbb{P}^{1} \mathrm{~s}$ ) in the fiber. Except for sporadic outliers, a Kodaira fiber is characterized in terms of an affine simple Lie algebra $\widehat{\mathfrak{g}}$, with a rational curve $F_{i}$ associated to each simple root $\alpha_{i}$

$$
\alpha_{i} \quad \leftrightarrow \quad F_{i} \cong \mathbb{P}^{1}, \quad i=0, \ldots, \operatorname{rank}(\mathfrak{g})
$$

Fibering $F_{i}$ over $W_{u}$ results in divisors, the so-called Cartan divisors,

$$
F_{i} \hookrightarrow D_{i} \rightarrow W_{u}
$$

which are in one-to-one correspondence with the Cartans of $\widehat{\mathfrak{g}}$. For simply-laced Lie algebras $\mathfrak{g}$, the fibral curves $F_{i}$ intersect with the Cartans in the negative Cartan matrix

$$
D_{i} \cdot F_{j}=-\mathcal{C}_{i j}^{\widehat{\mathfrak{g}}} .
$$

An example is shown in figure 3 , where in codimension one above $u=0$ the fiber is of type $I I^{*}$ or $\widehat{\mathfrak{g}}=\widehat{\mathfrak{e} 8}$.

For a non-simply-laced Lie algebra $\mathfrak{g}$, the $F_{i}$ can be multiple copies of $\mathbb{P}^{1}$, and the associated $D_{i}$ is reducible as well. In this case, the intersection matrix is given by [81]

$$
D_{i} \cdot F_{j}=-\frac{2\langle\alpha, \alpha\rangle_{\max }\left\langle\alpha_{i}, \alpha_{j}\right\rangle}{\left\langle\alpha_{i}, \alpha_{i}\right\rangle\left\langle\alpha_{j}, \alpha_{j}\right\rangle},
$$

where $\alpha_{i}$ is the root that corresponds to the curve $F_{i}$ and $\langle\alpha, \alpha\rangle_{\max }$ is the maximal length of the roots in the Lie algebra $\mathfrak{g}$. We will show an example of $\mathfrak{g}_{2}$ in section 3.4.

\subsubsection{Codimension two: flat and non-flat resolutions}

Consider next the situation when two such codimension one singular fibers collide. Let $W_{u}$ and $W_{v}$ be components of the discriminant along $u=0$ and $v=0$, respectively, with associated Kodaira fibers of type $\widehat{\mathfrak{g}}_{u / v}$. There are essentially two cases to distinguish:

$$
\begin{aligned}
\text { Minimal: } & \operatorname{ord}_{u=v=0}(f)<4 \quad \text { or } \quad \operatorname{ord}_{u=v=0}(g)<6 \\
\text { Non-Minimal: } & \operatorname{ord}_{u=v=0}(f, g, \Delta) \geq(4,6,12) .
\end{aligned}
$$

In the Tate form, non-minimality is characterized by $\operatorname{ord}_{u=v=0}\left(b_{i} ; \Delta\right) \geq(1,2,3,4,6 ; 12)$. In the minimal case the fiber corresponds to what normally is interpreted as bifundamental matter, and above the codimension two locus $u=v=0$ there is again a collection of rational curves, intersecting in a (possibly reduced) Kodaira singular fiber. A fiber resolution (and 
the associated smooth fibration) giving such codimension two fibers is called flat (the fiber dimension stays at complex one dimension). An in-depth analysis of the possible fiber types can be found in $[28,46,65,66,82-85]$. Here we will consider a simple example.

The simplest case arises for $u=0$ with an $I_{n}$ fiber, i.e., $\mathfrak{g}=\mathfrak{s u}(n)$, and $v=0$ with an $I_{1}$ (effectively a $\mathfrak{u}(1)$ ). The singular fiber in this case above $u=0$ is given in terms of a ring of $n$ rational curves $F_{i}, i=0, \ldots, n-1$. At $u=v=0$ one of these curves will become reducible and splits into two rational curves

$$
F_{i} \rightarrow C_{i}^{+}+C_{i+1}^{-}
$$

The fiber in codimension two corresponds in this situation to the Kodaira fiber $I_{n+1}$, and the $n+1$ rational curves intersect in the $\mathfrak{s u ( n + 1 )}$ affine Dynkin diagram. Moving away from the locus $u=v=0$ along the divisor $u=0$ can be thought of as a Higgsing $\mathfrak{s u}(n+1) \rightarrow \mathfrak{s u}(n) \oplus \mathfrak{u}(1)$. The curves $C_{i}^{ \pm}$carry charges under the Cartan divisors $D_{j}$ which in this case correspond to the fundamental $\mathbf{n}$ representation, consistent with this Higgsing. The sign \pm indicates whether the curve carries $\pm \lambda$ as charge vector, where $\lambda$ is a weight of the fundamental representation.

The situation of interest in this paper occurs when the collision is non-minimal. In this case the codimension two fiber has a resolution in terms of a non-flat fiber, i.e., the fiber above $u=v=0$ is not only a collection of curves, but also complex surfaces and so overall is not of complex dimension one. Alternatively we can resolve the base by successively blowing up the locus $u=v=0$. As this is more commonly done, we will focus here on describing the non-flat fiber resolution. An example is shown in figure 3, where $S$ is the non-flat surface component above $u=v=0$, arising from colliding an $\widehat{\mathrm{E}_{8}}$ and an $I_{1}$ fiber. $^{4}$ The surface $S$ contains a collection of curves that are part of the codimension one singular fiber (in the example shown in figure 3 , this is a subset of curves, which form an $\mathrm{E}_{7}$ subDynkin diagram of the codimension one affine $\mathrm{E}_{8}$ singular fiber). Non-flat fiber resolutions in codimension two have been studied systematically in [46], and more recently in [13, 29].

The codimension one fibral curves $F_{i}$ can split in the non-flat case as well, i.e., the rational curves in codimension two will be a collection of curves associated to the roots $F_{j}$ and weights $C_{i}^{ \pm}$- in figure $3, F_{1}$ splits into the two $(-1)$-curves $C^{ \pm}$, of which one is contained in the non-flat surface. In general there can be multiple surface components $S_{k}$ (the number of distinct values of $k$ depends on the precise rank enhancement in codimension two), which can in part contain the fibral curves.

As we have argued in section 2.2, the compact surface components $S_{j}$ obtained in this way will in general give rise to the $5 \mathrm{~d}$ gauge group, whereas the $(-2)$-curves $F_{i}$ of the codimension two fiber contained in $\mathcal{S}=\bigcup_{j} S_{j}$ determine the flavor symmetry of the SCFT that is obtained in the singular limit. We will refer these curves as flavor curves. While we come back to the associated 5d physics in more detail in the next section, we point out that the resolution process very easily produces many physically inequivalent (in 5d) geometries related via flop transitions. Crucially, these transitions also involve flops between compact divisors $S_{j}$ and non-compact divisors $D_{i}$, which from the perspective of

\footnotetext{
${ }^{4}$ For $I_{1}$ the codimension one fibration is generically smooth, and shown as a node.
} 


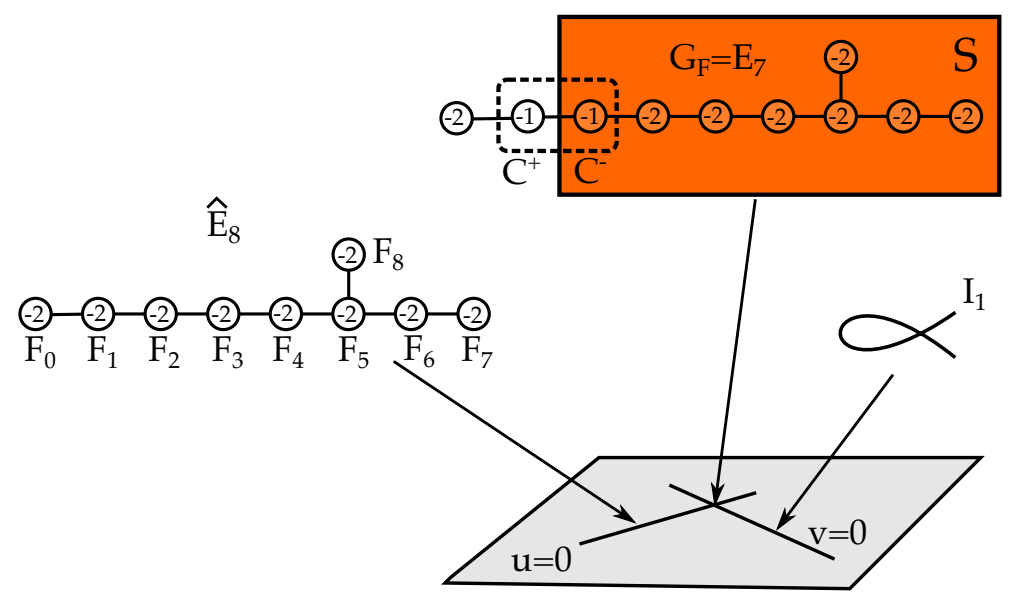

Figure 3. Rank one E-string: depicted is the collision of two codimension one singularities of type $\mathrm{E}_{8}$ and $I_{1}$, respectively, as well as the codimension two fiber including the non-flat component $S$. In codimension one, the fiber above $u=0$ is a collection of $(-2)$-curves $F_{i} \cong \mathbb{P}^{1}$ that are in one-to-one correspondence with the affine roots of $\mathrm{E}_{8}$. At the collision point $u=v=0$, one of these rational curves splits (here $F_{1} \rightarrow C^{+}+C^{-}$). The non-flat fiber component $S$ is a complex surface above the codimension two locus, which contains some of the $(-2)$-curves of the codimension one fiber - the so-called flavor curves. In the situation depicted, $S$ contains $F_{2}, \cdots, F_{8}$, which intersect in the $\mathrm{E}_{7}$ Dynkin diagram - we will refer to these curves in the following as flavor curves. Indeed, we will argue that they encode the flavor symmetry of the associated strongly coupled SCFT to be $\mathrm{E}_{7}$.

$\mathcal{S}$ is a contraction, i.e., a mass deformation in 5 d. In that way, fiber resolutions of elliptic threefolds with non-minimal singularities naturally produce different $5 \mathrm{~d}$ SCFTs associated to the same $6 \mathrm{~d}$ theory.

While most of our geometric examples are obtained from a non-flat resolutions, we stress that this is merely a convenient method to find smooth phases of a non-minimal elliptic singularity. For higher rank examples discussed in sections 3.4, we employ a combination of base and fiber blow-ups, resulting in compact surfaces $S_{j}$ which are both flat and non-flat. In fact, flatness of the $S_{j}$ with respect to the elliptic fibration is irrelevant for the geometric realization of 5d SCFTs. The only crucial part of our proposal is that we keep track of the flavor curves inside the compact surface $\mathcal{S}=\bigcup_{j} S_{j}$.

For 5d SCFTs descending from $6 \mathrm{~d}$ conformal matter theories, this is particularly simple, as the flavor curves are geometrically manifest. For other types of 6d SCFTs, such as models on fibrations with a singular base, making the flavor symmetry geometrically manifest require in general additional, non-trivial tunings of the generic Weierstrass model for these SCFTs [63, 64]. Moreover, certain 5d marginal theories may arise from outerautomorphism twists of 6d SCFTs, which geometrically corresponds to taking discrete quotients of the tensor branch geometries. Making the flavor curves explicitly manifest in such examples will require a detailed analysis beyond the scope of this paper. However, we will propose in section 4.4 a way to encode the SCFT-relevant data of rank two marginal theories that are quotient geometries. The results which agree perfectly with previous works $[6,12,24]$ will serve as additional evidence for the efficacy of our proposal. 
Importantly, these results are based on intuitions drawn from non-flat resolutions. Because of their importance, we will now briefly explain technical details of the fiber resolution procedure, following [46].

\subsubsection{Resolution of singular fibers}

Consider an elliptic fibration with singular locus given by $x_{1}=x_{2}=x_{3}=0$. We will denote the blow-up by the shorthand notation similar to [46]

$$
\left\{x_{1}, x_{2}, x_{3}, u_{i}\right\}: \quad x_{j}=u_{i} x_{j}^{\prime}, \quad j=1,2,3,
$$

where $x_{i}$ are placeholders for coordinates $x, y, u, u_{k}, k<i$, appearing in a (partially resolved) Tate model. The coordinate $u_{i}$ corresponds to the exceptional divisor of the resolution. After the blow-up, the new coordinates $\left[x_{1}^{\prime}, x_{2}^{\prime}, x_{3}^{\prime}\right]$ are treated as projective coordinates on a $\mathbb{P}^{2}$ and thus cannot vanish simultaneously. The singular locus $x_{1}=x_{2}=x_{3}=0$ is hence replaced by the exceptional divisor $u_{i}=0$, which corresponds to the aforementioned $\mathbb{P}^{2}$. The blow-up requires also performing a proper transform to keep the canonical class invariant, which amounts to dividing the Weierstrass equation by $u_{i}^{2}$.

Likewise, small resolutions will be denoted by $\left\{x_{1}, x_{2}, u_{i}\right\}$, which maps $x_{1}=x_{1}^{\prime} u_{i}$ and $x_{2}=x_{2}^{\prime} u_{i}$, where again the last entry $u_{i}$ corresponds to the exceptional section of the blow-up, where now $\left[x_{1}^{\prime}, x_{2}^{\prime}\right]$ are coordinates on an exceptional $\mathbb{P}^{1}$. The proper transform of the Weierstrass equation in this case is division by $u_{i}$. More generally, we also allow weighted blow-ups where the coordinates are transformed as $x_{j} \rightarrow u_{i}^{a_{j}} x_{j}, j=1,2,3$. They will play a role in the following in certain resolution sequences, for example the one in appendix D.6.2. When describing blow-ups, we will in the following always simply write $x_{i}$ instead of $x_{i}^{\prime}$, following a common abuse of notation.

For the situation at hand where we collide two codimension one singularities, the sequence of blow-ups contains the blow-ups of the codimension one fibers along $u=0$ and $v=0$, respectively. In the case of a non-minimal codimension two collision, we also need blow-ups of the type

$$
\left\{u_{i}, v_{j}, \delta_{k}\right\}: \quad u_{i} \rightarrow \delta_{k} u_{i}, v_{j} \rightarrow \delta_{k} v_{j}
$$

where $u_{i}$ and $v_{j}$ are exceptional sections of the respective codimension one resolutions. Here, $\delta_{k}=0$ corresponds to the non-flat fiber component $S_{k}$.

We will denote a complete resolution sequence, which smooths the Calabi-Yau threefolds, generally by $B U$. After such a resolution the proper transform of $u$ and $v$ are

$$
\begin{aligned}
& u=U\left(\prod_{i} u_{i}^{m_{i}^{(u)}}\right) \cdot\left(\prod_{j=1}^{r} \delta_{j}^{\xi_{i}^{(u)}}\right) \\
& v=V\left(\prod_{i} v_{i}^{m_{i}^{(v)}}\right) \cdot\left(\prod_{j=1}^{r} \delta_{j}^{\xi_{i}^{(v)}}\right),
\end{aligned}
$$

where $U=0$ and $V=0$ correspond to the Cartan divisor $D_{0}$ associated to the affine node of $\widehat{\mathfrak{g}}_{u}$ and $\widehat{\mathfrak{g}}_{v}$, respectively. While $m_{i}$ correspond to the well-known affine Dynkin labels of $\widehat{\mathfrak{g}}$, the $\xi_{i}$ coefficients are uniquely determined by the tensor branch information of the 
conformal matter theory. Suppose that the base coordinates $u$ and $v$ are described by $2 \mathrm{~d}$ toric rays $(1,0)$ and $(0,1)$ on a local $\mathbb{C}^{2}$ coordinate patch, then the surface component $\delta_{i}$ will correspond to exactly the ray $\left(\xi_{i}^{(u)}, \xi_{i}^{(v)}\right)$ on the 2 d base.

Different blow-up sequences correspond to distinct resolutions of the Calabi-Yau threefold, and have different 5d physical characteristics as we will explain in the next section. One particularly central quantity to characterize the physics, that is encoded in the the geometry of the surfaces $S_{k}$, is the intersection matrix $S_{k} \cdot D_{i}^{(\nu)} \cdot D_{j}^{(\mu)}$. However, to determine the flavor curves, i.e., the fibral curves are contained in the surfaces $S_{k}$, it is enough to quote the reduced intersection matrix

$$
n_{k, i, \nu}=S_{k} \cdot D_{i}^{(\nu)} \cdot D_{i}^{(\nu)}
$$

We can rewrite the total transform (2.30) in terms of the linear relation in integer homology involving the resolution divisors

$$
\pi^{-1}\left(W_{\nu}\right)=\sum_{i} m_{i}^{(\nu)} D_{i}^{(\nu)}+\sum_{i} \xi_{i}^{(\nu)} S_{i}
$$

where $\pi$ is the projection to the base. This linear relation is independent of the specific resolution.

Note that if we intersect $(2.32)$ for $\nu=u$ with $D_{j}^{(v)} \cdot D_{j}^{(v)}$, we obtain the relation

$$
\sum_{i} m_{i}^{(u)} D_{i}^{(u)} \cdot D_{j}^{(v)} \cdot D_{j}^{(v)}+\sum_{i} \xi_{i}^{(u)} S_{i} \cdot D_{j}^{(v)} \cdot D_{j}^{(v)}=\pi^{-1}\left(W_{u}\right) \cdot D_{j}^{(v)} \cdot D_{j}^{(v)} .
$$

Because $W_{u}$ intersects $W_{v}$ transversely in the base, we have $D_{j}^{(v)} \cdot \pi^{-1}\left(W_{u}\right) \cong F_{j}^{(v)}$, and the right-hand side can be simplified with $(2.24)$. If $F_{j}^{(v)}$ is a flavor curve, i.e., fully wrapped inside the surfaces $S_{j}$, then $D_{j}^{(v)} \cdot D_{i}^{(u)}=0$ for any $i$, as otherwise a component of $F_{j}^{(v)}$ would sit inside $D_{i}^{(u)}$. Hence, the criterion for $F_{j}^{(v)}$ to be a flavor curve is

$$
\sum_{i} \xi_{i}^{(u)} n_{i, j, v}=D_{j}^{(v)} \cdot F_{j}^{(v)}=-\frac{2\langle\alpha, \alpha\rangle_{\max }}{\left\langle\alpha_{j}, \alpha_{j}\right\rangle} \quad \text { (no summation) }
$$

which includes the case of non-simply laced algebras, see (2.25).

\section{5d SCFTs from conformal matter theories}

We begin our analysis by studying a particular class of 5d SCFTs, which descend from $6 \mathrm{~d}$ conformal matter theories. This will motivate the graphical approach using CFDs in the next section, which goes beyond the class of conformal matter descndants. Our starting point is a $6 \mathrm{~d} \mathcal{N}=(1,0)$ SCFT given in terms of a singular elliptic fibration with a nonminimal singularity in the fiber over $u=v=0$, which is given in terms of the collision of two non-compact codimension-one loci $W_{\nu} \subset B, \nu=u, v$ with Kodaira fibers of affine type $\widehat{\mathfrak{g}}_{\nu} \cdot{ }^{5}$ These $6 \mathrm{~d}$ SCFTs, so-called conformal matter theories, have flavor symmetry $G_{\mathrm{F}}^{(6 \mathrm{~d})}=G_{u} \times G_{v}$, which is manifest in the elliptic fibration.

\footnotetext{
${ }^{5}$ The base in these cases is always smooth. We discuss 6d SCFTs realized with singular bases in section 4.4. Moreover, we will not consider elliptic fibrations with non-trivial Mordell-Weil groups.
} 
As we explained in section 2.5, these singularities admit a crepant resolution in terms of a non-flat fibration. The non-flat fiber components are compact surfaces that are intersected by non-compact divisors $F_{i}^{(\nu)} \hookrightarrow D_{i}^{(\nu)}$ that resolve the codimension one singularities over $W_{\nu}$. Any such geometry defines via M-theory an effective 5 d theory on its Coulomb branch, which comes from a circle reduction with appropriate holonomies of the 6d SCFT. By determining all inequivalent non-flat resolutions, we can thus map out the entire network of descendant 5d SCFTs.

Since the $5 \mathrm{~d}$ theory arises from an $S^{1}$-reduction of the $6 \mathrm{~d}$ theory, we expect for the classical and the superconformal flavor symmetries, $G_{\mathrm{F}, \mathrm{cl}}$ and $G_{\mathrm{F}}$, respectively, the relation

$$
G_{\mathrm{F}}^{(6 \mathrm{~d})} \supset G_{\mathrm{F}} \supset G_{\mathrm{F}, \mathrm{cl}}
$$

Following the discussion in section 2.3, the 5d flavor symmetries are then determined by the collapse of the flavor curves $F_{i}^{(\nu)}$. This, and other physical properties largely follow from the discussion in the previous sections. As certain aspects are more manifest in the setup we discuss, we give a short summary adapted to this description.

\subsection{M-theory on non-flat fibrations: a dictionary}

Denote by $S_{j}, j=1, \ldots, r$ the non-flat fibers above $u=v=0$ and let again

$$
\mathcal{S}=\bigcup_{j=1}^{r} S_{j}
$$

be the reducible surface component.

Weakly coupled gauge description. The rank of the weakly coupled gauge group $G_{\text {gauge }}$ is given by the number of independent surface components in the non-flat fiber, i.e., $\operatorname{rank}\left(G_{\text {gauge }}\right)=r$. The precise gauge group is given by determining a ruling of the surfaces $f_{j} \hookrightarrow S_{j} \rightarrow \Sigma_{j}$, which allows a partial collapse $S_{j} \rightarrow \Sigma_{j}$, and the pairwise intersection pattern amongst the $S_{j}$, see section 2.2 .

By matching the prepotential (2.17) with the triple intersection numbers $S_{i} \cdot S_{j} \cdot S_{m}$, one can determine discrete data such as the Chern-Simons level $k$ for $\mathrm{SU}(K) \subset G_{\text {gauge }}$ or the number $M$ of mass deformations. If $G_{\text {gauge }}=\prod_{n=1}^{N} G_{\mathrm{G} ; n}$, where $G_{\mathrm{G} ; n}$ are simple gauge factors, then

$$
M=\operatorname{rank}\left(G_{\mathrm{F}, \mathrm{cl}}\right)+N=\operatorname{rank}\left(G_{\mathrm{F}}\right) .
$$

Field theoretically, the classical flavor symmetry $G_{\mathrm{F}, \mathrm{cl}}$ is entirely determined by the hypermultiplet spectrum. It can be verified geometrically, following section 2.3 , from the collapsed codimension one fibers when the compact surfaces are blow-down to curves, $S_{j} \rightarrow \Sigma_{j}$ [13]. Since the weakly coupled phase will play an underpart in this work, we will refer to the companion paper [31], where the classical flavor symmetry $G_{\mathrm{F}, \mathrm{cl}}$ becomes part of the main cast. 
Enhanced flavor symmetry from flavor curves. The central new aspect of our approach is that the superconformal flavor symmetry $G_{\mathrm{F}}$ is manifest from the geometry. This information can be determined, without explicitly knowing the effective theory, just from the numbers $n_{j, i, \nu}, \nu=u, v$, defined in (2.31). These numbers are an intersection-theoretic description of whether a codimension one fiber $F_{i}^{(\nu)}$ is contained inside $\mathcal{S}$, i.e., is a flavor curve or not. The numbers $n_{j, i, \nu}$ compute the degree of the normal bundle of the curve $S_{j} \cap D_{i}^{(\nu)}$ inside $S_{j}$.

For a Cartan node $F_{i}^{(\nu)}$ of $\mathfrak{g}_{\nu}$ inside $\mathcal{S}$ define (recall that $F_{i}$ is the fibral curve in the Cartan divisor $D_{i}$ )

$$
\begin{aligned}
& n\left(F_{i}^{(u)}\right)=\sum_{j=1} \xi_{j}^{(v)} n_{j, i, u}=\sum_{j=1} \xi_{j}^{(v)} S_{j} \cdot\left(D_{i}^{(u)}\right)^{2} \\
& n\left(F_{i}^{(v)}\right)=\sum_{j=1} \xi_{j}^{(u)} n_{j, i, v}=\sum_{j=1} \xi_{j}^{(u)} S_{j} \cdot\left(D_{i}^{(v)}\right)^{2} .
\end{aligned}
$$

where $\xi_{j}^{(\nu)}$ are defined in (2.30).

For a simply-laced Lie algebra $\mathfrak{g}_{\nu}$, a curve $F_{i}^{(\nu)}$ by $(2.34)$ is a flavor curve if $n\left(F_{i}^{(\nu)}\right)=-2$ - and the associated root contributes to the flavor symmetry. There are two instances to consider:

- If $n_{j, i, \nu}=-2$, then the fibral curve $F_{i}^{(\nu)}$ is irreducible in codimension two and is contained in $S_{j}$.

- If for $j \neq \ell, n_{j, i, \nu}=n_{\ell, i, \nu}=-1$, then the fibral curve $F_{i}$ is reducible, but is fully contained in $\mathcal{S}$. Its irreducible components of self-intersection -1 are contained in $S_{j}$ or $S_{\ell}$, respectively, and the root associated to $F_{i}^{(\nu)}$ is part of $G_{\mathrm{F}}$.

If on the other hand there is only one $j$ with $n_{j, i, \nu}=-1$, but $n_{j, k, \nu}=0$ for all $k \neq i$, then the curve $F_{i}^{(\nu)}$ is reducible, and only one of the split components (denoted by $C^{ \pm}$ in $(2.27))$ is contained in $\mathcal{S}$ with self-intersection $(-1)$. In this case the curve $F_{i}^{(\nu)}$ does not contribute to the non-abelian part of the strongly coupled flavor symmetry.

For the non-simply-laced case, the $F_{i}^{(\nu)}$ is a flavor curve if and only if (see (2.34))

$$
n\left(F_{i}^{(\nu)}\right)=-\frac{2\langle\alpha, \alpha\rangle_{\max }}{\left\langle\alpha_{i}, \alpha_{i}\right\rangle} .
$$

The Dynkin diagram of the non-abelian flavor symmetry $G_{\mathrm{F}, \mathrm{na}} \subset G_{\mathrm{F}}$ is read off from the graph that is formed by the flavor curves and is a subgraph of the affine Dynkin diagrams of $\hat{\mathfrak{g}}_{u}$ and $\hat{\mathfrak{g}}_{v}$. The abelian part $\mathrm{U}(1)^{s} \subset G_{\mathrm{F}}$ can be in most cases computed from knowing the mass deformations $M=\operatorname{rank}\left(G_{\mathrm{F}}\right)$ :

$$
s=\operatorname{rank}\left(G_{\mathrm{F}}\right)-\operatorname{rank}\left(G_{\mathrm{F}, \mathrm{na}}\right)=M-\operatorname{rank}\left(G_{\mathrm{F}, \mathrm{na}}\right) .
$$

Geometrically, these U(1)s correspond to $s$ independent linear combinations $D_{a}$ involving non-compact divisors, that intersect $\mathcal{S}$ non-trivially and are orthogonal to $G_{\mathrm{F}, \text { na }}$. In practice, we can compute $M$ as the rank of the intersection matrix between all independent 
divisors and curves of the form $D_{i}^{(\nu)} \cdot S_{j}$, after subtracting the rank of 5 d gauge group $r=\operatorname{rank}\left(G_{\text {gauge }}\right)$; see appendix D.2 for explicit examples. Note that since the intersections are all occurring locally inside $\mathcal{S}$, we can also identify a $\mathrm{U}(1)$ generator $D_{a}$ with the curves $D_{a} \cap \mathcal{S}$, and determine the $\mathrm{U}(1)$ charges as intersection numbers between curves in the surface $\mathcal{S}$. Such a representation of a flavor U(1) in terms of curves is in general not unique, due to linear relations amongst non-compact divisors.

Note that while we have assumed that the geometry realizes the full $6 \mathrm{~d}$ flavor symmetry as $G_{\mathrm{F}}^{(6 \mathrm{~d})}=G_{u} \times G_{v}$, it can be $G_{u} \times G_{v}$ is only a subgroup of $G_{\mathrm{F}}^{(6 \mathrm{~d})}$. In the latter case, the geometrically determined putative superconformal flavor group $G_{\mathrm{F}}$ can also be a subgroup of the actual, larger $5 \mathrm{~d}$ flavor group. In this case, the full flavor symmetry is obtained by computing the BPS states of a certain spin (see section 5), which will recombine into representations of a large flavor symmetry group $G_{\mathrm{F}}$. If this combination is indeed possible, the $5 \mathrm{~d}$ superconformal flavor group should enhance to $\widetilde{G}_{\mathrm{F}}$. Such examples are discussed in appendix D.6.1 and D.6.2. E.g., the rank one 5d SCFTs are described either by $\left(\mathrm{E}_{8}, I_{1}\right)$ or $\left(\mathrm{E}_{6}, \mathrm{SU}(3)\right)$ collisions. In the latter case, we only see the full flavor symmetry by considering the BPS states, whereas in the former it is manifest in geometry. This also reflects the situation in $6 \mathrm{~d}$ where the flavor symmetry is $G_{\mathrm{F}}^{(6 \mathrm{~d})}=\mathrm{E}_{8} \supset \mathrm{E}_{6} \times \mathrm{SU}(3)$. For the remaining part of this paper, we will assume that the singular elliptic fibration we start with already has the $6 \mathrm{~d}$ flavor symmetry manifest.

Relevant deformations and SCFT-trees. By shrinking a (-1)-curve that is contained in only one surface $S_{j} \subset \mathcal{S}$, the triple intersection number $S_{j}^{3}$ increases by one. Field theoretically, this corresponds to a relevant deformation of the SCFT (or mass deformation of the gauge theory) that triggers an RG-flow. Each contraction (or flop) of this type lowers $M=\operatorname{rank}\left(G_{\mathrm{F}}\right)$ by one, thus the resulting SCFT is a different one. What appears to be a curve contraction on $\mathcal{S}$ corresponds in the full smooth Calabi-Yau threefold to a birational map, where the collapsed (-1)-curve is flopped into a non-compact divisor. In this way we will determine all descendant SCFTs from a given marginal theory by flop transitions.

UV-dualities. There are also transitions in which a (-1)-curve $C$ inside $S_{j}$ is flopped into an adjacent $S_{k}$ as $C^{\prime}$. In this case the limiting SCFT does not change, as this does not change the overall structure of flavor curves (whether a flavor curve is irreducible or reducible in codimension two is immaterial as long as it is fully contained in the reducible surface $\mathcal{S}$ ). Such transformations neither change $G_{\mathrm{F}}$ nor $M$. In a field theory context, these flops between surface components inside $\mathcal{S}$ correspond to different weakly coupled gauge theory phases. For example, it can in rank two examples happen that a geometry with both a weakly coupled $\mathrm{SU}(3)_{\mathrm{G}}$ and $\mathrm{SU}(2)_{\mathrm{G}} \times \mathrm{SU}(2)_{\mathrm{G}}$ description only allows for an $\mathrm{SU}(3)_{\mathrm{G}}$ interpretation after such a flop (see [31] for more details). Nevertheless, these gauge theories are dual to each other in the sense of flowing to the same UV theory.

\subsection{Rank one classification from non-flat resolutions}

To see our proposal at work we start with the rank one 5d SCFTs, which are known to arise from circle reductions with flavor holonomies of the $6 \mathrm{~d}$ rank one E-string theory. The 


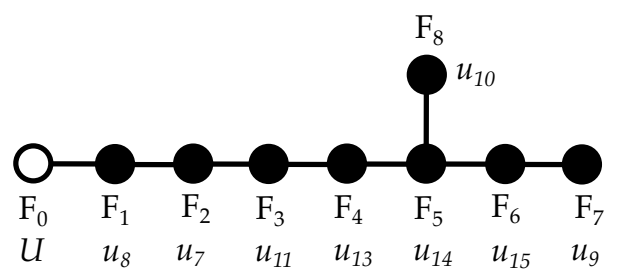

Figure 4. Affine $\mathrm{E}_{8}$ Dynkin diagram with labels corresponding to the exceptional sections $u_{i}$ and associated to the Cartan divisors $D_{k}$ that correspond to the simple roots $\alpha_{k}$. The labels $u_{i}$ refer to the exceptional sections of the blow-up.

weakly-coupled description is given by an $\mathrm{SU}(2)$ gauge theory with $N_{F}$ fundamental hypermultiplets, which has classical flavor symmetry $\mathrm{SO}\left(2 N_{F}\right)$ that at the UV fixed point enhances to $\mathrm{E}_{N_{F}+1}$. Geometrically, these SCFTs correspond to different M-theory compactifications on non-compact Calabi-Yau threefolds with canonical singularities, stemming from the collapse of a del Pezzo surface $\mathrm{dP}_{n}$ or the Hirzebruch surface $\mathbb{F}_{0} \cong \mathbb{P}^{1} \times \mathbb{P}^{1}[9,25]$.

It has been known that there are many other equivalent ways to engineer the same SCFTs, e.g., M-theory on a Calabi-Yau threefold with an $\mathbb{F}_{0}$ is considered to be equivalent to one with $\mathbb{F}_{2}$. The key difference in our description is that we will use such equivalences to make the superconformal flavor symmetry of the UV fixed points manifest within the geometry.

The $6 \mathrm{~d}$ E-string theory is obtained from an $\left(\mathrm{E}_{8}, I_{1}\right)$ (i.e., $\left.\left(I I^{*}, I_{1}\right)\right)$ non-minimal collision of Kodaira singularities, where the $\mathrm{E}_{8}$ associated with the $I I^{*}$ fibers encodes the $6 \mathrm{~d}$ flavor symmetry group. The $5 \mathrm{~d}$ rank one theories descend via flops from the so-called marginal geometry, from which all other theories are obtained by flop transitions. This corresponds to a particular resolution of this non-minimal singularity in terms of a non-flat fibration, where the surface component $S$ contains all fibral curves of the affine $\mathrm{E}_{8}$ of the $I I^{*}$ fiber.

The marginal theory has a surface omponent that is a generalized del Pezzo surface $\mathrm{gdP}_{9}$. While a $\mathrm{dP}_{n}$ contains a number of $(-1)$-curves as Mori cone generators, a $\mathrm{gdP}_{n}$ has rational generators with self-intersection $(-2)$ as well. Contracting the (-1)-curve in a $\operatorname{gdP}_{n}$ maps it to a $\operatorname{gdP}_{n-1}$ surface. A discussion of the geometry of these surfaces can be found in appendix C. It is from the $(-2)$-curves that are contained within the surfaces, that we read off the superconformal flavor symmetry: in the case of $\operatorname{gdP}_{n}$ surfaces, an $\mathrm{E}_{n}$ Dynkin diagram worth of rational (-2)-curves shrink in the UV limit, and furnish the flavor symmetry.

Non-flat resolutions of $\left(\mathbf{E}_{\mathbf{8}}, \boldsymbol{I}_{\mathbf{1}}\right)$ : the collision of a $I I^{*}$ Kodaira fiber transversally with an $I_{1}$ fiber has a simple description in terms of a Tate model for the elliptic fibration (2.21) with vanishing orders

$$
\operatorname{ord}_{u}\left(b_{i}\right)=(1,2,3,4,5), \quad \operatorname{ord}_{v}\left(b_{i}\right)=(0,0,0,0,1),
$$

which takes the form

$$
y^{2}+b_{1} u x y+b_{3} u^{3} y=x^{3}+b_{2} u^{2} x^{2}+b_{4} u^{4} x+b_{6} u^{5} v .
$$


At the codimension two locus in the base $u=v=0$ the vanishing orders of the $b_{i}$ are $(1,2,3,4,6)$, which is equivalent to the non-minimality condition in the Weierstrass model

$$
\operatorname{ord}_{u=v=0}(f, g, \Delta)=(4,6,12) \text {. }
$$

We now turn to deriving the resolutions of the singular model (3.8). In any resolution, the Cartan divisors $D_{i}$ associated to the $\mathrm{E}_{8}$ affine roots are given in terms of the sections

$$
\left(U, u_{8}, u_{7}, u_{11}, u_{13}, u_{14}, u_{15}, u_{9}, u_{10}\right) \equiv\left(D_{0}, \cdots, D_{8}\right)
$$

with the ordering shown in figure 4 . These intersect with the fibral curves $F_{i}$ in the negative affine $\mathrm{E}_{8}$ Cartan matrix,

$$
F_{i} \cdot D_{j}=-\mathcal{C}^{\widehat{\mathrm{E}_{8}}}=\left(\begin{array}{ccccccccc}
-2 & 1 & 0 & 0 & 0 & 0 & 0 & 0 & 0 \\
1 & -2 & 1 & 0 & 0 & 0 & 0 & 0 & 0 \\
0 & 1 & -2 & 1 & 0 & 0 & 0 & 0 & 0 \\
0 & 0 & 1 & -2 & 1 & 0 & 0 & 0 & 0 \\
0 & 0 & 0 & 1 & -2 & 1 & 0 & 0 & 0 \\
0 & 0 & 0 & 0 & 1 & -2 & 1 & 0 & 1 \\
0 & 0 & 0 & 0 & 0 & 1 & -2 & 1 & 0 \\
0 & 0 & 0 & 0 & 0 & 0 & 1 & -2 & 0 \\
0 & 0 & 0 & 0 & 0 & 1 & 0 & 0 & -2
\end{array}\right) .
$$

This is depicted in figure 4 .

$\mathbf{g d P}_{\mathbf{9}}$ : in the first model, the entire affine $\mathrm{E}_{8}$ worth of rational curves is contained within the compact surface $S$. This requires blowing up first the non-minimal locus $u=v=0$ in the base

$$
u \rightarrow U \delta, \quad v \rightarrow V \delta
$$

We will argue in section 4 that after this blow-up, the compact surfaces contain all fibral curves of the codimension one fibers.

After this blow-up, the locus $u=v=0$ is removed and replace by the curve $\{\delta=0\} \subset$ $B_{2}$. The Tate model still has the same form

$$
y^{2}+b_{1} U x y+b_{3} U^{3} y=x^{3}+b_{2} U^{2} x^{2}+b_{4} U^{4} x+b_{6} U^{5} V,
$$

but no longer has any non-minimal locus.

The model is still singular. The following blow-up sequence generates all the $\mathrm{E}_{8}$ Cartan divisors in (3.10)

$$
\begin{aligned}
& \left\{\left\{x, y, U, u_{1}\right\},\left\{x, y, u_{1}, u_{2}\right\},\left\{y, u_{2}, u_{3}\right\},\left\{y, u_{1}, u_{3}, u_{4}\right\},\right. \\
\operatorname{gdP}_{9}: \quad\left\{y, u_{1}, u_{5}\right\},\left\{u_{1}, u_{3}, u_{6}\right\},\left\{u_{1}, u_{4}, u_{7}\right\},\left\{u_{1}, u_{5}, u_{8}\right\},\left\{u_{2}, u_{3}, u_{9}\right\},\left\{u_{3}, u_{4}, u_{10}\right\}, & \\
& \left.\left\{u_{4}, u_{6}, u_{11}\right\},\left\{u_{3}, u_{6}, u_{12}\right\},\left\{u_{6}, u_{10}, u_{13}\right\},\left\{u_{10}, u_{12}, u_{14}\right\},\left\{u_{3}, u_{12}, u_{15}\right\}\right\} .
\end{aligned}
$$




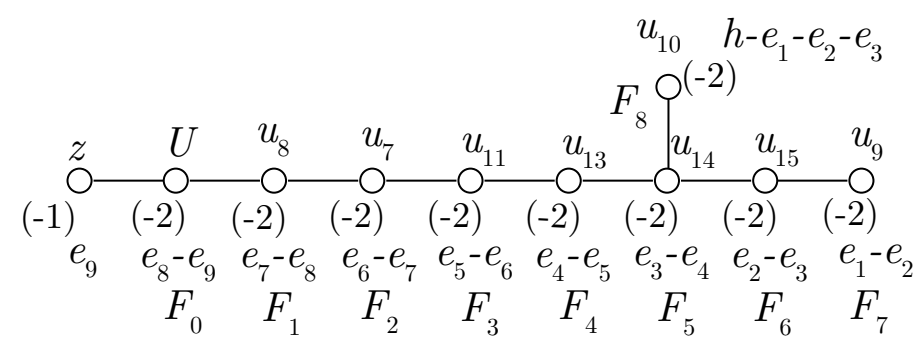

Figure 5. The negative curves on the vertical divisor $\operatorname{gdP}_{9}$, where the numbers in the brackets denote the self-intersection numbers and the letter $u_{i}$ or $z$ indicates the intersection of that divisor in the resolved Calabi-Yau with $S=\mathrm{gdP}_{9}$. We also denote the expression of each curve in terms of the standard basis of the Picard group $h$ and $e_{i}$ of a rational surface.

The configuration of curves on the $\mathrm{gdP}_{9}$ is shown in figure 5 , and we can read off the following intersection numbers:

$$
S \cdot D_{i} \cdot D_{i}=(-2,-2,-2,-2,-2,-2,-2,-2,-2),(i=0, \ldots, 8) .
$$

Furthermore we can compute

$$
S^{3}=0 \quad \Rightarrow \quad h^{1,1}(S)=10-S^{3}=10,
$$

consistent with the fact that $S$ is a nine-fold blow-up of a $\mathbb{P}^{2}$.

We can now either apply different blow-up sequences and obtain other surface components $S$, which contain a different subset of the $\mathrm{E}_{8}$ fibral curves - this is detailed in appendix D.1.1 - or we apply consecutively flop transitions to the $(-1)$-curves. The resulting tree of geometries connected by blow-ups or flops is summarized in table 1. This lists both the geometry of the surface $S$, which are generalized del Pezzo surfaces $\operatorname{gdP}_{n}$ or Hirzebruch surfaces $\mathbb{F}_{n}$. Furthermore we determine the intersection numbers $n_{i} \equiv S \cdot D_{i} \cdot D_{i}$ i.e., (2.31), which determine the flavor symmetry. In the present context, whenever a fibral curve $F_{i}$ with $F_{i}^{2}=-2$ is contained in the surface $S$, it contributes to the strongly coupled flavor symmetry. The curves for which $n_{i}=-1$ are reducible in codimension two and split. The associated root is not part of the flavor symmetry $G_{\mathrm{F}}$.

The key here is that the geometry manifestly encodes the strongly coupled flavor symmetry, as well as the complete flop chain descending from the marginal theory, which in the SCFT language corresponds to mass deformations and subsequent RG-flows to another UV fixed point. Needless to say, this is in complete agreement with the known rank one theories and their strongly coupled flavor symmetries $[9,25]$.

We conclude the discussion of rank one geometries by noting that there are alternative starting points, or marginal theories. E.g., the rank one collision $\left(\mathrm{E}_{6}, \mathrm{SU}(3)\right)$ also gives rise to the $6 \mathrm{~d}$ E-string. In appendix D.6.1 we consider resolutions of this collision. The maximal flavor symmetry present at the superconformal point is also correctly obtained from these models. However, it may not be manifest in these cases as the full enhancement, and only becomes apparent by combining the BPS states into representations of a higher rank group. This is in particular the case for the models with $\mathrm{E}_{8}$ and $\mathrm{E}_{7}$ flavor symmetry, which in 


\begin{tabular}{|c|c|c|c|}
\hline Geometry of $S$ & Intersections $S \cdot D_{i} \cdot D_{i}$ & Codim 2 Fiber & SCFT Flavor $G_{\mathrm{F}}$ \\
\hline $\operatorname{gdP}_{9}$ (Marginal) & $(-2,-2,-2,-2,-2,-2,-2,-2,-2)$ & (1)-O-O-O-O-O- & \\
\hline $\operatorname{gdP}_{8}$ & $(-1,-2,-2,-2,-2,-2,-2,-2,-2)$ & (1)-1(1) $0-0$ & $\mathrm{E}_{8}$ \\
\hline $\operatorname{gdP}_{7}$ & $(0,-1,-2,-2,-2,-2,-2,-2,-2)$ & $0+1-0-0$ & $\mathrm{E}_{7}$ \\
\hline $\operatorname{gdP}_{6}$ & $(0,0,-1,-2,-2,-2,-2,-2,-2)$ & $0-0+0$ & $\mathrm{E}_{6}$ \\
\hline $\operatorname{gdP}_{5}$ & $(0,0,0,-1,-2,-2,-2,-2,-2)$ & $\mathrm{O}-\mathrm{O}-\mathrm{O}$ & $\mathrm{SO}(10)$ \\
\hline $\operatorname{gdP}_{4}$ & $(0,0,0,0,-1,-2,-2,-2,-2)$ & $\mathrm{O}-\mathrm{O}-\mathrm{O}-\mathrm{O}$ & $\mathrm{SU}(5)$ \\
\hline $\operatorname{gdP}_{3}$ & $(0,0,0,0,0,-1,-2,-2,-2)$ & $\mathrm{O}-\mathrm{O}-\mathrm{O}-\mathrm{O}-\mathrm{O}$ & $\mathrm{SU}(2) \times \mathrm{SU}(3)$ \\
\hline $\operatorname{gdP}_{2}$ & $(0,0,0,0,0,0,-1,-2,-1)$ & $0-0-0-0,0 \% 0$ & $\mathrm{SU}(2) \times \mathrm{U}(1)$ \\
\hline $\mathbb{F}_{1}$ & $(0,0,0,0,0,0,0,-1,0)$ & $0-0-0-0-0-0 \div 0$ & $\mathrm{U}(1)$ \\
\hline $\operatorname{gdP}_{1} \cong \mathbb{F}_{2}$ & $(0,0,0,0,0,0,0,-2,0)$ & $0-0-0-0-0-0 \div 0-0-0 \div 0$ & $\mathrm{SU}(2)$ \\
\hline $\mathbb{P}^{2}$ & $(0,0,0,0,0,0,0,0,1)$ & $0-0-0-0-0-0 \div 0-0-0-0$ & - \\
\hline
\end{tabular}

Table 1. Geometries for rank one 5d SCFT: the table contains the geometry of the non-flat surface component $S$ in the elliptic fibration, the intersection of $S$ with the Cartans $D_{i}, i=0, \cdots, 8$ of the $\mathrm{E}_{8}$ fiber; the codimension two fiber, where the dotted lines show the splitting of the codimension one rational curves of the $I I^{*}$ fiber that become reducible. Futhermore, the marked nodes are contained in the non-flat fiber $S$. Full colored nodes that remain irreducible in codimension two correspond to $(-2)$ curves in the $I I^{*}$ fiber and contribute to the flavor symmetry of the SCFT, $G_{\mathrm{F}}$. The last column gives the flavor symmetry, which can be read off from the fiber.

this alternative description would not be manifest, and only is seen by computing the BPS states and their representations, which combine into multiplets of the higher rank flavor symmetries. In this sense, the marginal theory we consider in this section, i.e., $\left(\mathrm{E}_{8}, I_{1}\right)$, captures all of these symmetries complete manifestly within the geometric resolution, and is thus preferred.

\subsection{Rank two: non-flat resolutions}

The next application of our method is to the geometries that result in rank two theories. The non-flat fiber resolutions have two surface components, which in M-theory give rise to the Cartans of the gauge group (if there is weakly coupled gauge theory description). An example is shown in figure 6. As for rank one, we extract the flavor symmetries of the strongly coupled SCFT from the rational curves that are contained in the surface components of the fiber. 


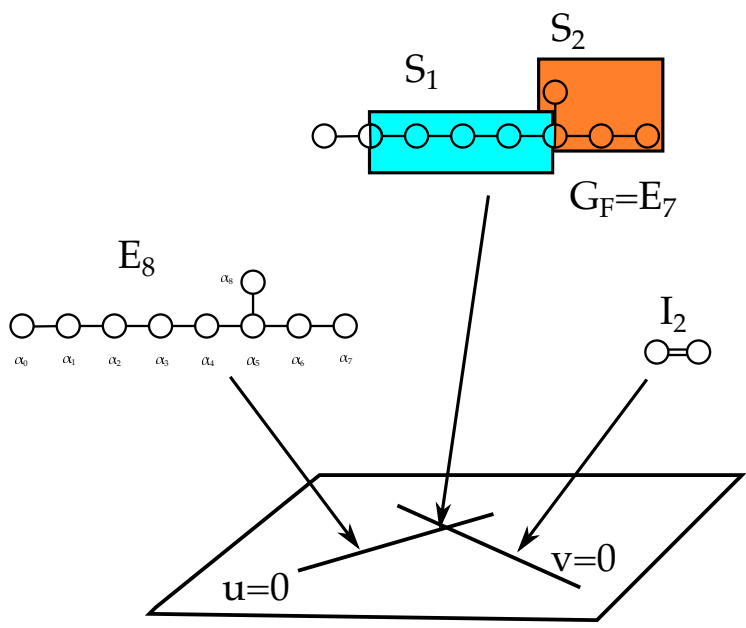

Figure 6. Cartoon of a codimension two fiber for the rank two E-string. $S_{i}$ are the two surface components of the non-flat fiber in codimension two. The rational curves (circles) that are fully contained in the surfaces contribute to the non-abelian flavor symmetry, which in the shown example is $G_{\mathrm{F}}=\mathrm{E}_{7}$.

We will provide a systematic exploration of all rank two theories in section 4 . Here, we will focus on two aspects: determine the geometries associated to the marginal theories and to point out some new features that occur in higher rank, e.g., the existence of resolutions that result in the same superconformal theories, i.e., the geometric avatar of different gauge theory phases for the same SCFT.

This will be exemplified in concrete resolution geometries, but we will pass to a more streamlined description in section 4 , where equivalence classes of resolutions will be characterized in terms of CFDs (combined fiber diagrams). The equivalence classes will in particular contain resolution geometries, which correspond to the same SCFTs and are simply different resolutions associated to gauge theory phases for the same UV fixed point. The CFDs, as we emphasized before, encode the essential information of the non-flat resolutions, such as the rational curves associated to the flavor symmetries hat are contained in the non-flat fiber components, and allow for a systematic determination of all $5 \mathrm{~d}$ descendants of a $6 \mathrm{~d}$ SCFT.

The two main marginal theories (whose descendants map out most of the rank two SCFTs) are the rank two E-string and the $\left(D_{5}, D_{5}\right)$ conformal matter theory. The latter is equivalently represented in terms of a collision between an $D_{10}$ with a transverse $I_{1}$, which is what we will consider in the following. They are given in terms of the Tate models (2.21) with vanishing orders

$$
\begin{array}{rll}
\left(D_{10}, I_{1}\right): & \operatorname{ord}_{U=0}\left(b_{i}\right)=(1,1,5,5,10), & \operatorname{ord}_{V=0}\left(b_{i}\right)=(0,1,0,0,0) \\
\left(\mathrm{E}_{8}, I_{2}\right): & \operatorname{ord}_{U=0}\left(b_{i}\right)=(1,2,3,4,5), & \operatorname{ord}_{V=0}\left(b_{i}\right)=(0,0,1,1,2) .
\end{array}
$$

\subsubsection{Non-flat resolutions of $\left(\mathrm{E}_{8}, \mathrm{SU}(2)\right)$}

Consider the rank two E-string geometry that is the codimension two non-minimal collision of $\mathrm{E}_{8}$ along $u=0$, with an $I_{2}(\mathrm{SU}(2))$ singular fiber along $v=0$. Our focus will be on 
determining the blow-up for the marginal theory, as well as providing some examples. The explicit resolutions can be found in the appendix D.2.

The geometry for the marginal theory is obtained as a non-flat resolution in appendix D.2.1, where the full derivation is explained. Here we only summarize it in terms of the reduced triple intersection matrix

\begin{tabular}{c|ccccccccc|cc|cc}
$S_{i} \cdot D_{j}^{2}$ & $D_{0}$ & $D_{1}$ & $D_{2}$ & $D_{3}$ & $D_{4}$ & $D_{5}$ & $D_{6}$ & $D_{7}$ & $D_{8}$ & $D_{0}^{\mathrm{SU}(2)}$ & $D_{1}^{\mathrm{SU}(2)}$ & $S_{1}$ & $S_{2}$ \\
\hline$S_{1}$ & -2 & -1 & 0 & 0 & 0 & 0 & 0 & 0 & 0 & -2 & 0 & 6 & 0 \\
$S_{2}$ & 0 & -1 & -2 & -2 & -2 & -2 & -2 & -2 & -2 & 0 & -1 & -2 & 1 \\
\hline$n\left(F_{j}\right)$ & -2 & -2 & -2 & -2 & -2 & -2 & -2 & -2 & -2 & -2 & -2 & - & -
\end{tabular}

Here $n\left(F_{j}\right)$ is defined in (3.4). The Cartan divisors of the affined $\mathrm{E}_{8}$ and $\mathrm{SU}(2)$ are intersected with the two surface components $S_{1}$ and $S_{2}$, as $n\left(F_{j}\right)$. Note that in the case of $\left(\mathrm{E}_{8}, \mathrm{SU}(2)\right)$, there are non-trivial multiplicities (see (2.30))

$$
\xi_{1}^{\mathrm{E}_{8}}=1, \quad \xi_{2}^{\mathrm{E}_{8}}=2, \quad \xi_{1}^{\mathrm{SU}(2)}=\xi_{2}^{\mathrm{SU}(2)}=1,
$$

hence the entries $S_{2} \cdot\left(D_{i}^{\mathrm{SU}(2)}\right)^{2}$ need to be multiplied by $\xi_{2}=2$ in the bottom line of (3.18), in order to read off the correct flavor curves.

We see that the curves at the intersection of the non-compact Cartan divisors and surface components are all $(-2)$ curves, so that all fibral curves associated to the affine roots of $\mathrm{E}_{8} \times \mathrm{SU}(2)$ are contained within the reducible surface $\mathcal{S}$ - the hallmark of the marginal geometry.

We can now determine all descendant geometries/SCFTs by flops, which will be the subject of section 4. Here we should consider a few more examples of non-flat resolutions to point out some new effects that occur in higher rank. Again the details of the resolutions are explained in appendix D.2.2. The key characteristic is the reduced triple intersection number. We consider three example blow-ups, for which this is given by

$$
\begin{aligned}
& \mathrm{BU}_{1}^{\left(\mathrm{E}_{8}, \mathrm{SU}(2)\right)} \text { : } \\
& \begin{array}{r|ccccccccc|cc|cc}
S_{i} \cdot D_{j}^{2} & D_{0} & D_{1} & D_{2} & D_{3} & D_{4} & D_{5} & D_{6} & D_{7} & D_{8} & D_{0}^{\mathrm{SU}(2)} & D_{1}^{\mathrm{SU}(2)} & S_{1} & S_{2} \\
\hline S_{1} & 0 & -1 & -2 & -2 & -2 & -1 & 0 & 0 & 0 & 0 & 0 & 4 & -4 \\
S_{2} & 0 & 0 & 0 & 0 & 0 & -1 & -1 & 0 & -2 & 0 & 1 & 2 & 7 \\
\hline n\left(F_{j}\right) & 0 & -1 & -2 & -2 & -2 & -2 & -1 & 0 & -2 & 0 & 2 & - & -
\end{array} \\
& \mathrm{BU}_{2}^{\left(\mathrm{E}_{8}, \mathrm{SU}(2)\right)} \text { : } \\
& \begin{array}{c|ccccccccc|cc|cc}
S_{i} \cdot D_{j}^{2} & D_{0} & D_{1} & D_{2} & D_{3} & D_{4} & D_{5} & D_{6} & D_{7} & D_{8} & D_{0}^{\mathrm{SU}(2)} & D_{1}^{\mathrm{SU}(2)} & S_{1} & S_{2} \\
\hline S_{1} & 0 & 0 & 0 & 0 & 0 & 0 & 0 & 0 & 0 & 0 & 0 & 8 & 0
\end{array} \\
& \begin{array}{c|ccccccccc|cc|cc}
S_{2} & 0 & -1 & -2 & -2 & -2 & -2 & -1 & 0 & -2 & 0 & 1 & -2 & 3 \\
\hline n\left(F_{j}\right) & 0 & -1 & -2 & -2 & -2 & -2 & -1 & 0 & -2 & 0 & 2 & - & -
\end{array} \\
& \mathrm{BU}_{3}^{\left(\mathrm{E}_{8}, \mathrm{SU}(2)\right)} \text { : } \\
& \begin{array}{c|ccccccccc|cc|cc}
S_{i} \cdot D_{j}^{2} & D_{0} & D_{1} & D_{2} & D_{3} & D_{4} & D_{5} & D_{6} & D_{7} & D_{8} & D_{0}^{\mathrm{SU}(2)} & D_{1}^{\mathrm{SU}(2)} & S_{1} & S_{2} \\
\hline S_{1} & 0 & -1 & -2 & -2 & -2 & -1 & 0 & 0 & 0 & 0 & 0 & 4 & -4
\end{array} \\
& \begin{array}{c|ccccccccc|cc|cc}
S_{2} & 0 & 0 & 0 & 0 & 0 & -1 & -2 & -1 & -2 & 0 & 0 & 2 & 6 \\
\hline n\left(F_{j}\right) & 0 & -1 & -2 & -2 & -2 & -2 & -2 & -1 & -2 & 0 & 0 & - & -
\end{array}
\end{aligned}
$$




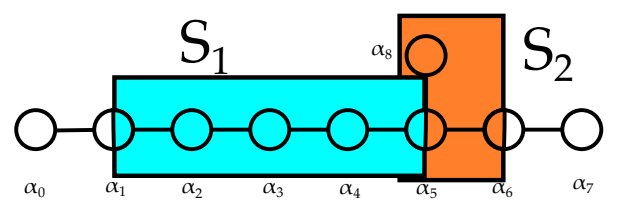

(a) $\mathrm{BU}_{1}^{\left(\mathrm{E}_{8}, \mathrm{SU}(2)\right)}$ : wrapping of codimension one Fiber by surfaces $S_{i}$.

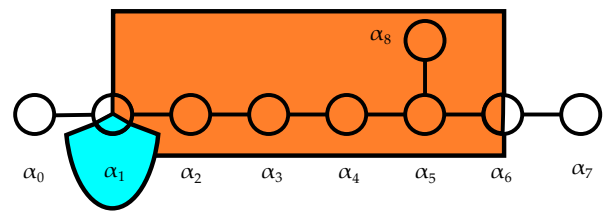

(c) $\mathrm{BU}_{2}^{\left(\mathrm{E}_{8}, \mathrm{SU}(2)\right)}$ : wrapping of codimension one Fiber by surfaces $S_{i}$.

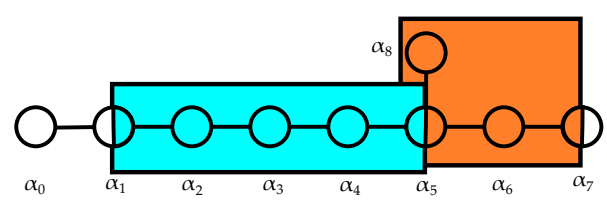

(e) $\mathrm{BU}_{3}^{\left(\mathrm{E}_{8}, \mathrm{SU}(2)\right)}$ : wrapping of codimension one Fiber by surfaces $S_{i}$.

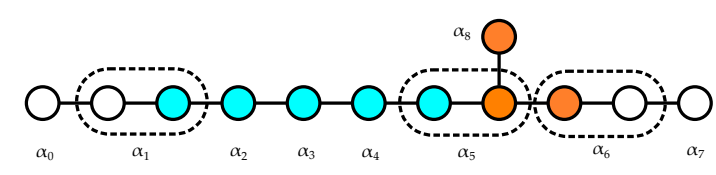

(b) $\mathrm{BU}_{1}^{\left(\mathrm{E}_{8}, \mathrm{SU}(2)\right)}$ : codimension two Fiber.

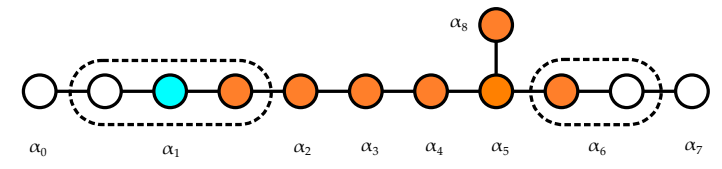

(d) $\mathrm{BU}_{2}^{\left(\mathrm{E}_{8}, \mathrm{SU}(2)\right)}$ : codimension two Fiber.

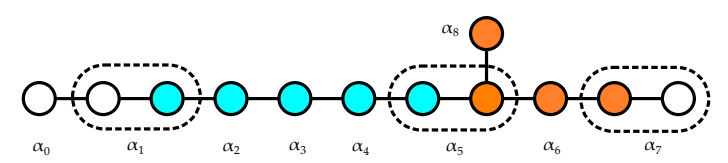

(f) $\mathrm{BU}_{3}^{\left(\mathrm{E}_{8}, \mathrm{SU}(2)\right)}$ : codimension two Fiber.

Figure 7. Example Blowups for the Rank two E-string $\mathrm{BU}_{i}^{\left(\mathrm{E}_{8}, \mathrm{SU}(2)\right)}$. We show both the fiber of the $\mathrm{E}_{8}$ and the curves that are contained in the surfaces components $S_{1}$ (turquois) and $S_{2}$ (orange) of the non-flat fiber, as well as, in the second row of each model, the actual codimension two fiber with all the irreducible fiber components and their intersections.

The codimension two fibers for these blow-ups are shown in figure 7. By considering the triple intersection number with the reducible surface $\mathcal{S}$, we can read off the stronglycoupled flavor symmetries as follows

$$
\begin{aligned}
G_{\mathrm{F}}^{\mathrm{BU}_{1}}=G_{\mathrm{F}}^{\mathrm{BU}_{2}} & =\mathrm{SU}(6) \times \mathrm{U}(1) \\
G_{\mathrm{F}}^{\mathrm{BU}_{3}} & =\mathrm{SO}(12) \times \mathrm{U}(1)
\end{aligned}
$$

Note that the resolutions $\mathrm{BU}_{1}$ and $\mathrm{BU}_{2}$ result in the same UV fixed point, although the geometric resolutions are distinct. The resolution geometries describe two distinct weaklycoupled gauge theory descriptions, of the same SCFT. It is this equivalence between resolutions that we will modd out by in the subsequent discussion of CFDs in section 4, and condense the geometric description to one that does not have such redundancies in the characterization of $5 \mathrm{~d}$ SCFTs.

\subsubsection{Non-flat resolutions of $\left(D_{10}, I_{1}\right)$}

The second class of non-flat resolutions in rank two that we will consider is the collision $D_{10}$ with a non-generic $I_{1}$ Kodaira fiber with the following vanishing orders in the Tate model

$$
\operatorname{ord}_{U=0}\left(b_{i}\right)=(1,1,5,5,10), \quad \operatorname{ord}_{V=0}\left(b_{i}\right)=(0,1,0,0,0) .
$$




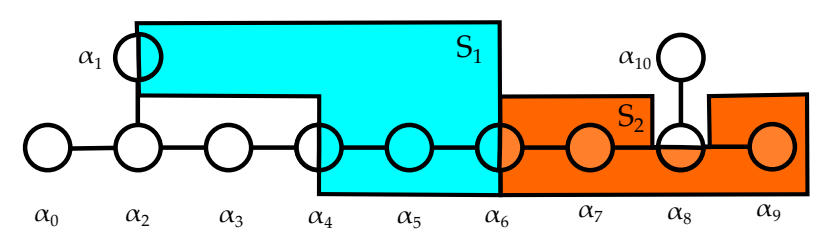

(a) $\mathrm{BU}_{1}^{\left(D_{10}, I_{1}\right)}$ : wrapping of codimension one Fiber by surfaces $S_{i}$.

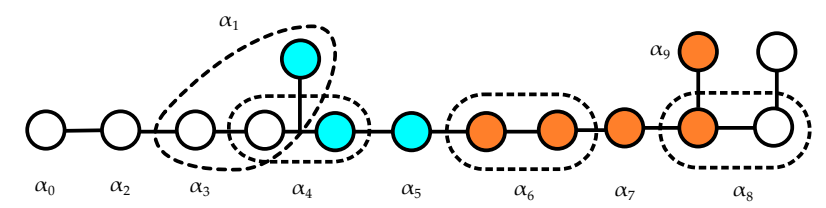

(b) $\mathrm{BU}_{1}^{\left(D_{10}, I_{1}\right)}$ : codimension two Fiber where dotted lines indicate the splitting of the codimension one fiber components.

Figure 8. Example blow-up $\mathrm{BU}_{1}^{\left(D_{10}, I_{1}\right)}$. The first row shows the wrapping of the codimension one fiber. The second row gives the full codimension two fiber in terms of irreducible curves.

Here we tuned the vanishing order of $b_{6}$ to trivially satisfy the split condition of the Kodaira fiber for $\mathrm{SO}(20)$.

First we summarize what we find in appendix D.3.1 for the marginal geometry. The reduced triple intersection matrix is ${ }^{6}$

\begin{tabular}{c|ccccccccccc|c|cc}
$S_{i} D_{j}^{2}$ & $D_{0}$ & $D_{1}$ & $D_{2}$ & $D_{3}$ & $D_{4}$ & $D_{5}$ & $D_{6}$ & $D_{7}$ & $D_{8}$ & $D_{9}$ & $D_{10}$ & $D_{0}^{I_{1}}$ & $S_{1}$ & $S_{2}$ \\
\hline$S_{1}$ & 0 & 0 & 0 & 0 & 0 & 0 & 0 & 0 & 0 & 0 & 0 & 0 & 8 & 4 \\
$S_{2}$ & -2 & -2 & -2 & -2 & -2 & -2 & -2 & -2 & -2 & -2 & -2 & 0 & -6 & -2 \\
\hline$n\left(F_{i}\right)$ & -2 & -2 & -2 & -2 & -2 & -2 & -2 & -2 & -2 & -2 & -2 & 0 & - & -
\end{tabular}.

In this resolution, the rational curves of the $D_{10}$ affine fiber are all contained within one surface component already. This geometry will define the marginal CFD in section 4 .

Let us consider also an example resolution of a non-marginal theory - the details are given in appendix D.3.2. The reduced triple intersection numbers are

\begin{tabular}{r|ccccccccccc|c|cc}
$S_{i} \cdot D_{j}^{2}$ & $D_{0}$ & $D_{1}$ & $D_{2}$ & $D_{3}$ & $D_{4}$ & $D_{5}$ & $D_{6}$ & $D_{7}$ & $D_{8}$ & $D_{9}$ & $D_{10}$ & $D_{0}^{I_{1}}$ & $S_{1}$ & $S_{2}$ \\
\hline$S_{1}$ & 0 & 0 & 0 & 0 & -1 & -2 & -1 & 0 & 0 & 0 & 0 & 4 & 6 & -2 \\
$S_{2}$ & 0 & 0 & 0 & 0 & 0 & 0 & -1 & -2 & -1 & -2 & 0 & 2 & 0 & 6 \\
\hline$n\left(F_{j}\right)$ & 0 & 0 & 0 & 0 & -1 & -2 & -2 & -2 & -1 & -2 & 0 & 6 & - & -
\end{tabular}

from which we can read off the strongly-coupled flavor symmetry

$$
G=\mathrm{SU}(4) \times \mathrm{SU}(2) \times \mathrm{U}(1)
$$

The corresponding codimension two fiber is shown in figure 8. Note that the abelian part of the flavor symmetry is obtained by considering the full triple intersection matrix, as discussed in appendix D.3.2.

\footnotetext{
${ }^{6}$ For $\left(D_{2 k}, I_{1}\right)$ (or equivalently, $\left(D_{k}, D_{k}\right)$ theories, the multiplicities $\xi_{i}=1$ for all $i$.
} 
An alternative starting point is the non-minimal $\left(D_{5}, D_{5}\right)$ collision, which will be discussed in appendix D.6.2. The models obtained from the resolution of this collision will give rise to equivalent models, however to see the full flavor symmetry, one needs to compute the BPS states and repackage them in terms of higher rank groups. Our $\left(D_{10}, I_{1}\right)$ starting point does not require this, and manifestly encodes the flavor symmetries geometrically.

\subsection{Higher rank conformal matter}

Starting with any non-minimal codimension two collision of two codimension one singular fibers of type $\mathfrak{g}_{u}$ and $\mathfrak{g}_{v}$ can be analyzed in the fasion described in this section.

An infinite class of such $5 \mathrm{~d}$ theories at arbitrary rank descending from $6 \mathrm{~d}$

$$
\left(D_{k}, D_{k}\right), \quad \text { or } \quad\left(D_{2 k}, I_{1}\right)
$$

minimal conformal matter were discussed in [8], as well as $\left(\mathrm{E}_{6}, \mathrm{E}_{6}\right)$ conformal matter for which we determined all descendant SCFTs.

A systematic exploration of higher rank will appear in [30]. Here we will give a higher rank conformal matter example, $\left(\mathrm{E}_{8}, G_{2}\right)$ for which we also determine the complete set of daughter 5d SCFTs in section 4. A more systematic analysis of the higher rank cases will follow in that section as well, where we determine a more combinatorial way of generating all the geometries.

Consider the collision of $I I^{*}$ and $I_{3}$ Kodaira fibers associated to the $\left(\mathrm{E}_{8}, \mathrm{SU}(3)\right)$ conformal matter, which has vanishing orders

$$
\operatorname{ord}_{u=0}\left(b_{i}\right)=(1,2,3,4,5), \quad \operatorname{ord}_{v=0}\left(b_{i}\right)=(0,1,1,2,3) .
$$

The 5d SCFTs obtained from this have rank 4 and the resolution is given in appendix D.4. The reduced triple intersection matrix of the Cartan divisors $D_{i}^{\mathfrak{g}}$ of $\mathrm{E}_{8}$ and $\mathrm{SU}(3)$, respectively, with the four non-flat fiber components $S_{i}$ are

$$
\begin{aligned}
& \mathrm{BU}^{\left(\mathrm{E}_{8}, \mathrm{SU}(3)\right)}: \\
& \begin{array}{c|ccccccccc|ccc|cccc}
S \cdot D_{j}^{2} & D_{0} & D_{1} & D_{2} & D_{3} & D_{4} & D_{5} & D_{6} & D_{7} & D_{8} & D_{0}^{I_{3}} & D_{1}^{I_{3}} & D_{2}^{I_{3}} & S_{1} & S_{2} & S_{3} & S_{4} \\
\hline S_{1} & 0 & 0 & 0 & 0 & 0 & 0 & 0 & 0 & 0 & 0 & -1 & -1 & 6 & -2 & -2 & 0 \\
S_{2} & 0 & 0 & 0 & 0 & 0 & 0 & 0 & -1 & 0 & 0 & -1 & -1 & -2 & 4 & -4 & -2 \\
S_{3} & 0 & -1 & -2 & -1 & 0 & 0 & 0 & 0 & 0 & 0 & 0 & 0 & 0 & 0 & 6 & -2 \\
S_{4} & 0 & 0 & 0 & -1 & -2 & -2 & -2 & -1 & -2 & 0 & 0 & 0 & 0 & 0 & 0 & 4 \\
\hline n\left(F_{j}\right) & 0 & -1 & -2 & -2 & -2 & -2 & -2 & -2 & -2 & 0 & -2 & -2 & - & - & - & -
\end{array}
\end{aligned}
$$

Note that although the $\left(\mathrm{E}_{8}, \mathrm{SU}(3)\right)$ conformal matter has the following $\xi_{i}$ coefficients

$$
\xi_{1}^{\mathrm{E}_{8}}=\xi_{2}^{\mathrm{E}_{8}}=1, \quad \xi_{3}^{\mathrm{E}_{8}}=2, \quad \xi_{4}^{\mathrm{E}_{8}}=3, \quad \xi_{i}^{\mathrm{SU}(3)}=1, \quad i=1, \cdots, 4,
$$

they only affect the triple intersection numbers $S_{3} \cdot\left(D_{i}^{I_{3}}\right)^{2}$ and $S_{4} \cdot\left(D_{i}^{I_{3}}\right)^{2}$, which are zero in this case.

The wrapped components of the fiber and codimension two fiber is shown in figure 9 . The strongly coupled flavor symmetry for the SCFT from this point of view is

$$
G_{\mathrm{F}} \supset \mathrm{E}_{7} \times \mathrm{SU}(3) .
$$




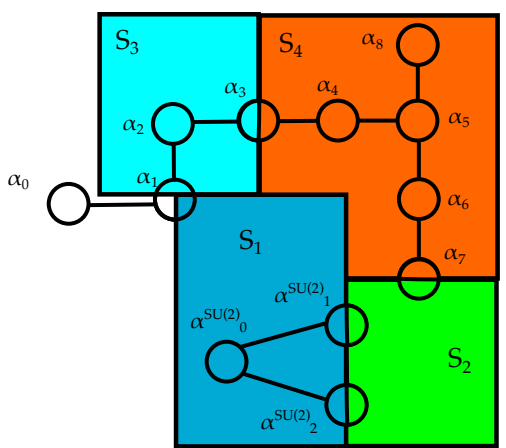

(a) $\mathrm{BU}^{\left(\mathrm{E}_{8}, \mathrm{SU}(3)\right)}$ : wrapping of codimension one Fiber by surfaces $S_{i}$.

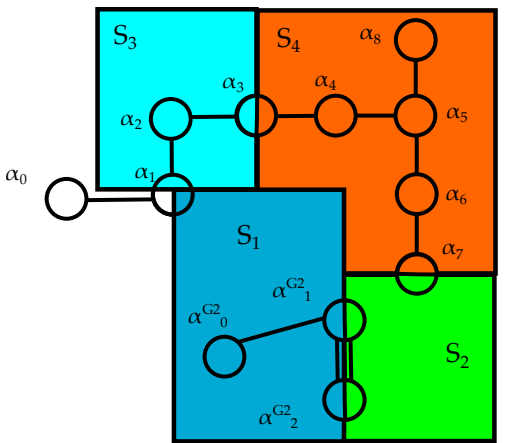

(b) $\mathrm{BU}^{\left(\mathrm{E}_{8}, G_{2}\right)}$ : wrapping of codimension one Fiber by surfaces $S_{i}$.

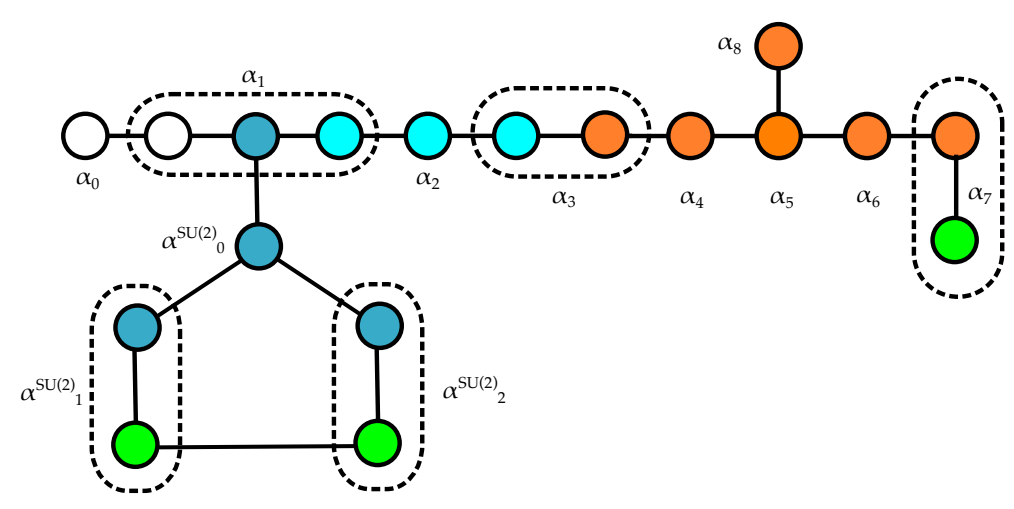

(c) $\mathrm{BU}^{\left(\mathrm{E}_{8}, \mathrm{SU}(3)\right)}$ : codimension two Fiber where dotted lines indicate the splitting of the codimension one fiber components.

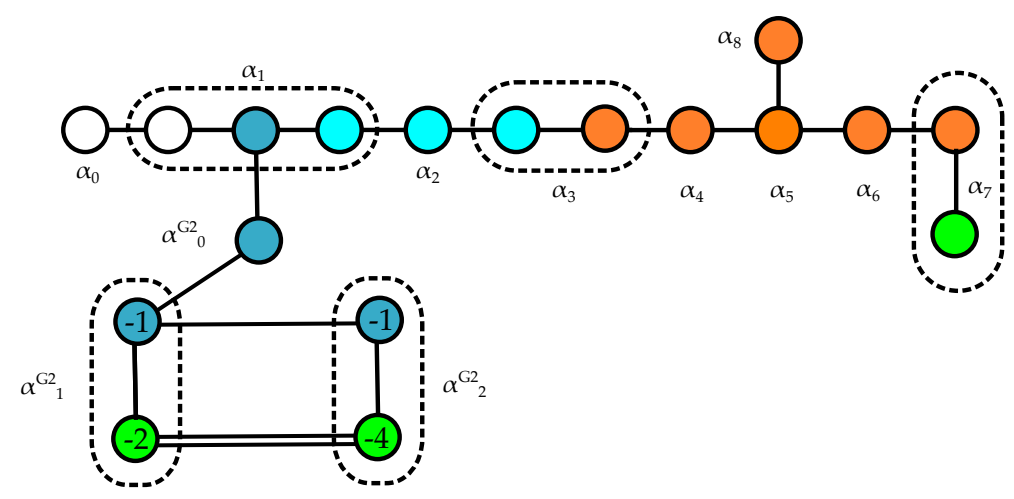

(d) $\mathrm{BU}^{\left(\mathrm{E}_{8}, G_{2}\right)}$ : codimension two Fiber where dotted lines indicate the splitting of the codimension one fiber components.

Figure 9. Example blow-up for $\left(\mathrm{E}_{8}, \mathrm{SU}(3)\right)$ and $\left(\mathrm{E}_{8}, G_{2}\right)$. The gauge theory description has rank 4 gauge group, corresponding to the four surface components $S_{i}$. The first image shows the affine $\mathrm{E}_{8}$ and $\mathrm{SU}(3)$ and how the $S_{i}$ wrap these (half-wrapping corresponding to self-intersection $(-1)$, full wrapping to $(-2))$, see (D.59). 
In fact there is an alternative starting point, where the full $6 \mathrm{~d}$ flavor symmetry $\left(\mathrm{E}_{8}, G_{2}\right)$ is manifest [86].

With some minor changes we can generalize this to $\left(\mathrm{E}_{8}, G_{2}\right)$, i.e., the collision of $I I^{*}$ with $I_{1}^{n s}$ (non-split $I_{1}^{*}$ ). The vanishing orders change to

$$
\operatorname{ord}_{v=0}\left(b_{i}\right)=(0,1,1,2,3)
$$

The reduced triple intersection matrix for this case is, with the coefficients $\xi_{i}$ as in (3.29) and (3.4)

$$
\begin{aligned}
& \mathrm{BU}^{\left(\mathrm{E}_{8}, G_{2}\right)}: \\
& \begin{array}{c|ccccccccc|ccc|cccc}
S \cdot D_{j}^{2} & D_{0} & D_{1} & D_{2} & D_{3} & D_{4} & D_{5} & D_{6} & D_{7} & D_{8} & D_{0}^{G_{2}} & D_{1}^{G_{2}} & D_{2}^{G_{2}} & S_{1} & S_{2} & S_{3} & S_{4} \\
\hline S_{1} & 0 & 0 & 0 & 0 & 0 & 0 & 0 & 0 & 0 & 0 & -1 & -2 & 6 & -2 & -2 & 0 \\
S_{2} & 0 & 0 & 0 & 0 & 0 & 0 & 0 & -1 & 0 & 0 & -1 & -4 & -2 & 4 & -4 & -2 \\
S_{3} & 0 & -1 & -2 & -1 & 0 & 0 & 0 & 0 & 0 & 0 & 0 & 0 & 0 & 0 & 6 & -2 \\
S_{4} & 0 & 0 & 0 & -1 & -2 & -2 & -2 & -1 & -2 & 0 & 0 & 0 & 0 & 0 & 0 & 4 \\
\hline n\left(F_{j}\right) & 0 & -1 & -2 & -2 & -2 & -2 & -2 & -2 & -2 & 0 & -2 & -6 & - & - & - & -
\end{array}
\end{aligned}
$$

The fiber (and containments within the non-flat fibers) is shown in figure 9 . So here the flavor symmetry at the strongly coupled point is

$$
G_{\mathrm{F}}=\mathrm{E}_{7} \times G_{2} .
$$

Clearly the realization in terms of the collision with $G_{2}$ captures the full flavor symmetry in 5d. In the next section we will discuss using our proposed graphical presentation the higher rank generalizations to $\left(\mathrm{E}_{8}, \mathrm{SU}(m)\right), m>3$, as well as the $\left(\mathrm{E}_{n}, \mathrm{E}_{n}\right)$ conformal matter theories systematically. In these cases the maximal flavor symmetry is manifest already in the $6 \mathrm{~d}$ realizations.

\section{5d SCFTs from graphs}

We now turn to reformulating the geometric description of 5d SCFTs that descend from $6 \mathrm{~d}$ SCFTs in a succinct way, which we already introduced in our recent paper [8], in terms of a graph-theoretic tool, the combined fiber diagram (CFD). A CFD characterizes a 5d SCFT, its superconformal flavor symmetry and mass deformations. Furthermore, it enables a systematic and comprehensive derivation of all descendant SCFTs from a given marginal theory.

\subsection{Combined Fiber Diagrams (CFDs)}

A CFD was defined in [8] as a graph, whose vertices $C_{i}$ are curves and whose edges are given by intersection numbers $m_{i, j}=C_{i} \cdot C_{j}$ between the curves. Furthermore, vertices carry labels $\left(n_{i}, g_{i}\right)$, which are the self-intersection number and genus of the curve associated to the vertex.

We will now explain how a CFD can be associated to any crepant resolution of an elliptically fibered Calabi-Yau threefold $Y$. 
Denote by $B$, the non-compact base of $Y$, where there is a local coordinate patch $(u, v)$. Furthermore, consider the elliptic fibration with Kodaira fibers of type $\mathfrak{g}_{u}$ above $u=0$ and $\mathfrak{g}_{v}$ above $v=0\left(\mathfrak{g}_{\nu}\right.$ can either refer to the algebra associated to the fiber or the fiber type). At $u=v=0$ let there be a non-minimal singularity, i.e., in terms of the Weierstrass model $\operatorname{ord}(f, g, \Delta)_{u=v=0} \geq(4,6,12)$. Consider a crepant blow-up, BU $: \tilde{Y} \rightarrow Y$ of the threefold that introduces $r$ compact surface components $S_{i}$ with

$$
\mathcal{S}=\bigcup_{i=1}^{r} S_{i} .
$$

This corresponds to a rank $r$ d theory. There are a number of non-compact divisors that intersect $\mathcal{S}$, including the Cartan divisors $D_{i}^{(\nu)}, \nu=u, v$, for the codimension one singularities.

Denote by $F_{k}$ all the fibral curves that are complete intersections between $\mathcal{S}$ and noncompact divisors, and are entirely contained within $\mathcal{S}$, we denote these by

$$
\mathcal{F}=\left\{C=D_{i}^{(\nu)} \cdot \mathcal{S} ; C \subset \mathcal{S}\right\} .
$$

These are the flavor curves. They are (a subset of) the rational curves associated to simple roots of the Lie algebras $\mathfrak{g}_{u}$ and $\mathfrak{g}_{v}$. Note that a rational curve that is reducible in codimension two is contained in $\mathcal{F}$, if all its irreducible components are all contained in $\mathcal{S}$ (i.e., they may not be contained in one single surface component, but in the reducible surface $\mathcal{S}$ ). For the precise conditions of a fully wrapped Cartan divisor, see the "Enhanced Flavor Symmetry" section in section 3.1. The CFD is effectively the set of Mori cone generators of the reducible surface $\mathcal{S}$. More precisely:

Definition 4.1 (CFD associated to a Crepant Resolution). Given a resolution BU of a non-minimal singular Weierstrass model, with the compact (reducible) surface $\mathcal{S}=\cup_{k} S_{k}$, the associated $C F D_{\mathrm{BU}}$ is a graph whose vertices are the curves $C \subset \mathcal{S}$, including:

1. The flavor curves $F_{i} \subset \mathcal{F}$ generating the non-abelian flavor symmetry, which are marked (usually colored green).

2. The rational $(-1)$-curves with normal bundle $\mathcal{O}(-1) \oplus \mathcal{O}(-1)$ that can be flopped outside of $\mathcal{S}$.

3. The other curves generating the Mori cone of $\mathcal{S}$, which are not intersection curves between $S_{i}$ and $S_{j}$.

We label each vertex by a pair of integers $(n, g)$, encoding its self-intersection number $n$ and the genus $g$. For the $\mathcal{O}(-1) \oplus \mathcal{O}(-1)$ curve in case 2., they are labelled by $(n, g)=$ $(-1,0)$. For the flavor curves $F_{i}$ in case 1 , , they are usually labeled as $(n, g)=(-2,0)$ unless there is a surface component $S_{j}$ with a non-trivial multiplicity $\xi_{j}>1$, which contains both the flavor curve $F_{i}$ and a $(-1)$-curve connected to $F_{i}$.

Two vertices $C_{i}$ and $C_{j}$ are connected by

$$
m_{i, j}=C_{i} \cdot C_{j}
$$

edges if they intersect at $m_{i, j}$ points in $\mathcal{S}$. 
Conventions: the label $g_{i}$ is generically omitted when $g_{i}=0$. Furthermore, marked vertices have, unless noted otherwise, $\left(n_{i}, g_{i}\right)=(-2,0)$, which again is often omitted.

The marked vertices form a sub-graph, which we identify with the Dynkin diagram of the non-abelian part of the superconformal flavor symmetry.

As examples consider the rank two E-string resolutions $\mathrm{BU}_{1}^{\left(\mathrm{E}_{8}, \mathrm{SU}(2)\right)}$ and $\mathrm{BU}_{2}^{\left(\mathrm{E}_{8}, \mathrm{SU}(2)\right)}$, see (D.30) and (D.38), respectively. The reduced intersection matrices (3.20) show that these models differ only in terms of how the $(-2)$ and $(-1)$ curves are distributed among the two surface components $S_{i}$, but the overall structure of $(-2)$ and $(-1)$ curves in the fiber that are contained in some non-flat surface are the same. Equivalently, the intersections with $\mathcal{S}$ and the Cartan divisors is the same in both cases. Hence, the associated CFDs (and SCFTs) are identical:

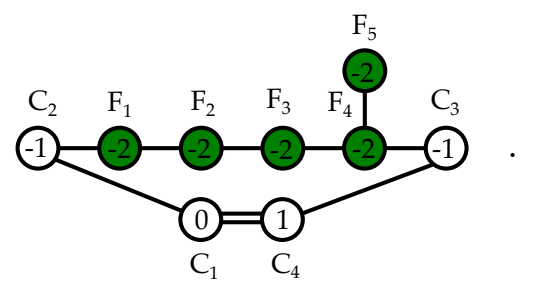

The marked, green vertices are the (-2)-curves that are contained in $S_{1} \cup S_{2}$, and determine the non-abelian part of the SCFT flavor symmetry to be $\mathrm{SU}(6)$. The white curves have self-intersections as indicated by the numbers in the nodes. In triple intersection matrices for $S_{i}$ (D.31), the corresponding (-2)- and (-1)-curves are boxed in. The curves $C_{1}$ and $C_{4}$ with self-intersection 0 and 1 inside the $\mathcal{S}$ are double-boxed, which are originally components of the fiber that come from the $I_{2}$ singular fiber above $v=0$. Note that the curve $C_{4}$ can be expressed as a linear combination of other curves:

$$
C_{4}=2 C_{2}+2 F_{1}+2 F_{2}+2 F_{3}+2 F_{4}+F_{5}+C_{3},
$$

and is not an indepedent Mori cone generator.

The advantage of the description in terms of CFDs compared to the fibers is two-fold: a CFD encodes the non-abelian part of the flavor symmetry manifestly in terms of the marked vertices, which form a sub-graph, which is the Dynkin diagram of the non-abelian part of the flavor symmetry of the SCFT, $G_{F, n a}$. For the abelian part U(1) ${ }^{s}$, the number of $\mathrm{U}(1)$ factors should still be computed by (3.6), with the number of mass deformations $M$. For the descendants of a given marginal CFD, this effectively equals to the number of white nodes minus a fixed number, see the details in section 4.4 .

Furthermore, we can transition between CFDs, which correspond in the geometry to contractions of rational curve with self-intersection $C_{i} \cdot C_{i}=-1$ (which do not correspond flavor curves). In (4.4) there are two $(-1)$-curves that can be contracted. The two descendant CFDs are obtained by the standard rules of blowing down $(-1)$-curves on a complex surface. In terms of the CFDs, which 'flops' of curves from within $\mathcal{S}$ to outside of $\mathcal{S}$ can be described in terms of simple graph-theoretic rules.

Definition 4.2 (CFD transitions). Let $C_{i}$ be an unmarked vertex with label $\left(n_{i}, g_{i}\right)=$ $(-1,0)$ of a $C F D$. We can define a new CFD, CFD' that is obtained by removing the vertex $C_{i}$ and updating the graph as follows: 
1. Let $C_{j}$ be a vertex in the original CFD with label $\left(n_{j}, g_{j}\right)$ with $C_{i} \cdot C_{j}=m_{i, j}$, then in $C F D^{\prime}$, the vertex $C_{j}$ is labeled by

$$
n_{j}^{\prime}=n_{j}+m_{i, j}^{2}, \quad g_{j}^{\prime}=g_{j}+\frac{m_{i, j}^{2}-m_{i, j}}{2} .
$$

2. If $C_{i} \cdot C_{j}=m_{i, j}$ and $C_{i} \cdot C_{k}=m_{i, k}$, and $C_{j} \cdot C_{k}=m_{j, k}$ then in $C F D^{\prime}$

$$
m_{j, k}^{\prime}=m_{j, k}+m_{i, j} m_{i, k}
$$

3. If $C_{i}$ intersects multiple curves $C_{i} \cdot C_{j}=m_{i, j}$, then rule 2. applies pairwise.

In particular if $n_{j}=-2$ with $m_{i, j}=1$, then $n_{j}^{\prime}=-1$. These rules determine the complete chain of descendant CFDs (and associated SCFTs). For instance for (4.4) the two descendants are

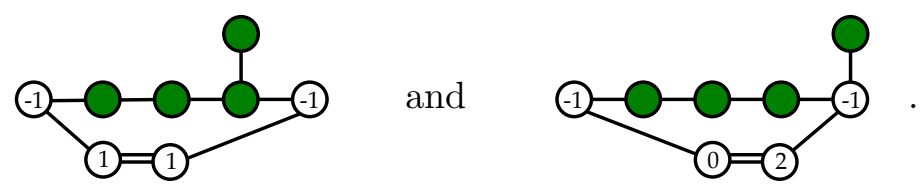

These have $\mathrm{SU}(5) \times \mathrm{U}(1)$ and $\mathrm{SU}(4) \times \mathrm{SU}(2) \times \mathrm{U}(1)$ strongly coupled flavor symmetry, respectively. We continue the CFD-transitions until a descendant CFD is reached, which does not have any vertices with $\left(n_{i}, g_{i}\right)=(-1,0)$. These are the endpoints of the CFD tree (and the RG-flows).

The descendant CFDs that we obtain in this way are irreducible in the sense, that the corresponding SCFTs do not factorize into SCFTs of lower ranks. Geometrically, this means that the CFD-transitions only correspond to flops of $(-1)$-curves, which are not at the intersection of two surface components. By construction, we do not include these curves into the CFDs.

\subsection{Starting points: marginal CFDs}

In light of these extremely simple rules governing CFDs and their transitions, it is important to determine the CFD for the marginal theories, from which all other descendant CFDs/SCFTs can be obtained by this simple, graph-theoretic operation. In this section, we explain how for a given codimension two non-minimal collision, one can compute the CFD for the marginal theory. The main characteristic of the marginal (or top) CFD is that its marked vertices form affine Dynkin diagrams for the codimension one singularities [8].

Consider the codimension two collision, as before, with $\left(\mathfrak{g}_{u}, \mathfrak{g}_{v}\right)$. To compute the marginal geometry and associated CFD, we follow the same strategy as in section 3. Before resolving the singularities in codimension one, we blow up the non-minimal locus on the base

$$
\left\{u, v, \delta_{1}\right\}: \quad u \rightarrow U \delta_{1}, \quad v \rightarrow V \delta_{1} .
$$

From a $6 \mathrm{~d}$ point of view this corresponds to a partial tensor branch. After this single blow-up in the base, we perform the resolution of the codimension one singular fibers as usual [46]. After the blow-ups, we have the following set of divisors: the vertical divisor

$$
S_{1}: \quad \delta_{1}=0
$$




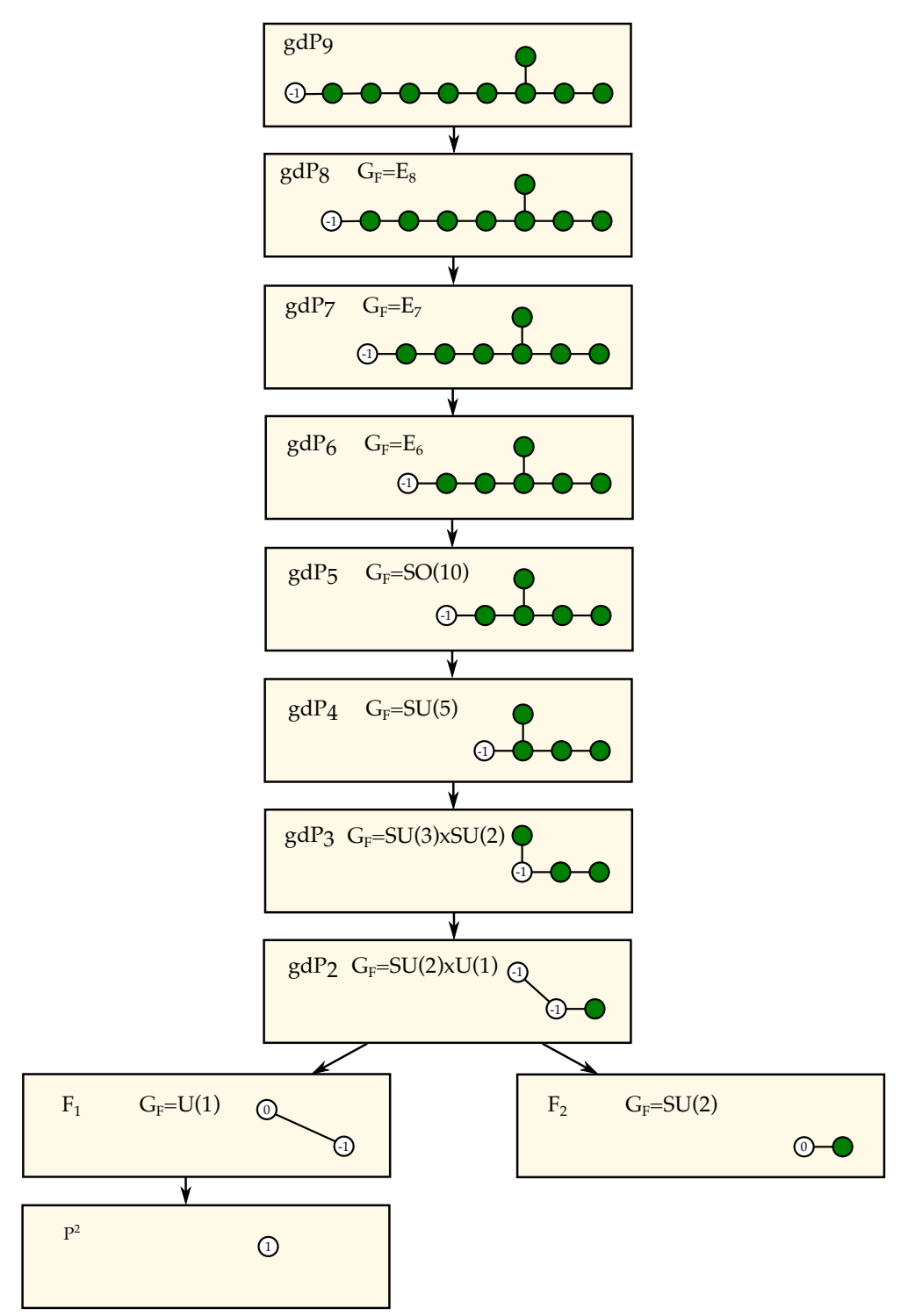

Figure 10. Rank one CFD tree starting with the marginal CFD, given by the $\mathrm{gdP}_{9}$, including all $(-2)$-curves (shown in green) as well as one $(-1)$-curve. The transitions are dictated by the rules in Definition 4.2. The strongly coupled flavor symmetry is denoted by $G_{\mathrm{F}}$ and the non-abelian part of it is read off from the (-2)-curves in the CFDs.

as well as the Cartan divisors for the codimension one singular fibers $D_{i}^{\mathfrak{g}_{\nu}}$. In fact, all the curves $S_{1} \cdot D_{i}^{\mathfrak{g}_{\nu}}$ for $\nu=u, v$ are already fully wrapped inside $S_{1}$. In general, the resulting Calabi-Yau threefold is still singular, and requires further small resolutions of the type

$$
\left\{u_{i}, \delta_{j}, \delta_{j+1}\right\} \quad \text { or } \quad\left\{v_{i}, \delta_{j}, \delta_{j+1}\right\}
$$

which result in $r$ surface components $S_{i}$, whose union $\mathcal{S}$ contains all codimension one rational curves. The CFD associated to this blow-up up is precisely the marginal CFD.

From this discussion, it is obvious that this marginal CFD will always contain the affine Dynkin diagram of $\mathfrak{g}_{u}$ and $\mathfrak{g}_{v}$ as subgraphs, as these are by construction always fully 
wrapped, and their vertices are marked (colored). The marginal CFD contains in addition curves with $n_{i} \geq-1$, which connect the two marked subgraphs.

For the rank one 5d SCFTs, the relevant starting point is the rank one E-string, and the blow-up for the marginal CFD already appeared in (3.12) combined with (3.14). For the rank two 5d SCFTs, the marginal CFDs are derived in appendices D.2.1 and D.3.1. In the following we will use these resolutions to determine all 5d SCFTs of rank one and two, and furthermore provide the marginal CFDs higher rank conformal matter theories.

\subsection{Rank one classification from CFDs}

For rank one 5d SCFTs, the marginal CFD corresponds precisely to the geometry of curves inside the generalized del Pezzo surface $\mathrm{gdP}_{9}$, shown in table 1 . In addition there is a $(-1)$ curve, which is also contained in the $\operatorname{gdP}_{9}$ and part of the generators of the Mori cone. In summary we find the marginal CFD for the rank one theories

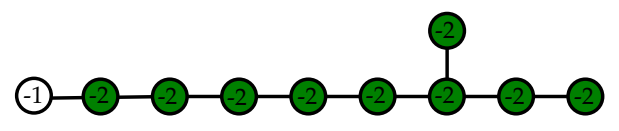

as proposed already in our earlier paper [8]. There is precisely one CFD-transition that can be applied to the vertex with $n=-1$. The complete rank one CFD-tree is shown in figure 10. Each descendant CFD is accompanied by the information about the superconformal flavor symmetry that is read off from the marked sub-graph, and the geometry of the non-flat fiber - as determined by direct resolutions in section 3.2. Needless to say, this tree agrees with the Seiberg theories and their mass deformations [9, 25] and the flavor symmetries predicted therein. Note that the CFD includes the theory that does not have a weakly-coupled gauge theory description (associated to the geometry $\mathbb{P}^{2}$ ).

It is useful to compare the CFD-tree with the complete fiber diagrams in section 3.2. The CFDs only capture the information that is relevant for the 5d SCFT - in particular, information about the additional curves that are unaffected by the singular limit is not retained. This makes these graphs particularly efficient.

\subsection{Rank two classification from CFDs}

To illustrate the power of the CFD approach, we next consider the rank two 5d SCFTs. The key is to find the marginal CFD for each of the starting points in $6 \mathrm{~d}$ listed in appendix A. This contains the codimension one $(-2)$-curves $F_{k}^{\mathfrak{g}_{u}}$ and $F_{l}^{\mathfrak{g}_{v}}$ in the fibers, but has to be determined from a resolution of the singular elliptic fibration, where the two surface components of the resolved geometry contain all these curves. We determine these resolutions in the appendices D.2.1 and D.3.1 for the rank two marginal theories.

There are seven starting points in total, two of which, $\left(\mathrm{E}_{8}, \mathrm{SU}(2)\right)$ and $\left(D_{10}, I_{1}\right)$, have a direct conformal matter origin. We infer the marginal CFDs for two further models, which arises as quotients from 6d. Starting with these marginal CFDs we generate the complete CFD-tree by transitions and confirm the tree-structure of SCFTs, that was obtained by independent methods in [6, 24]. Furthermore, we determine the superconformal flavor symmetries in all cases. 
1. $\left(D_{10}, I_{1}\right)$ : the marginal CFD for the $\left(D_{5}, D_{5}\right)$, or equivalently $\left(D_{10}, I_{1}\right)$ conformal matter theory is

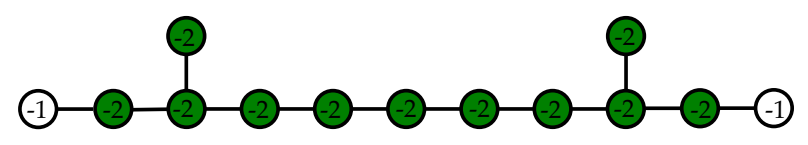

The complete fiber geometry was determined in figure 23 and the geometry of the surfaces in this non-flat resolution is

$$
S_{1} \cup S_{2}=\mathbb{F}_{1} \cup \mathrm{Bl}_{10} \mathbb{F}_{6} .
$$

Note that this is related after a number of flops to the surfaces in [6], which are $\mathrm{Bl}_{6} \mathbb{F}_{1} \cup \mathrm{dP}_{6}$. As emphasized repeatedly, however, our description is somewhat better suited for the purposes of reading off vital physical information of the SCFTs. This marginal CFD is extracted from figure 23, by first of all noting that the entire affine Dynkin diagram of $D_{10}$ is contained in the surface component $S_{2}$. These are all $(-2)$-curves and therefore marked vertices in the CFD. There is also a $(-1)$-curve $e_{1}$ on $S_{1}$, which connects the $(-2)$-node $F_{0}$ (labeled by $U$ ) and a $(-1)$-curve $e_{11}$ on $S_{2}$ that connects the node $F_{10}$ (labeled by $\left.u_{9}\right)$. These are unmarked $(n, g)=(-1,0)$ vertices, which will be key in determining the CFD-transition tree. There is no other Mori cone generator for $S_{1} \cup S_{2}$, hence the marginal CFD for the $\left(D_{10}, I_{1}\right)$ geometry is exactly the one in (4.13).

The complete CFD-tree that descends from this is shown in figure 11. We note down the (generally enhanced) superconformal flavor symmetry $G_{\mathrm{F}}$. The number of U(1) flavor symmetry factors is given by the number of white nodes minus two, and the generators of the U(1)s are chosen to be the white curves that do not intersect any of the green curves. Whenever an $\mathrm{SU}(3)$ gauge theory description exists we also specify the CS-level $k$. Theories with no gauge theory description are shaded in grey.

2. Rank two E-string $\left(\mathrm{E}_{8}, \mathrm{SU}(2)\right)$ : the marginal CFD for the rank two E-string is computed to be

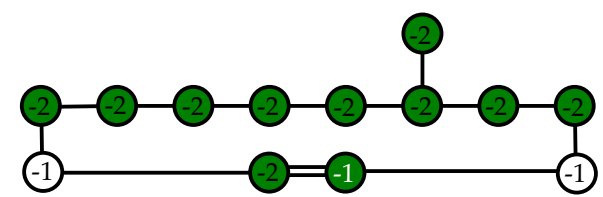

This was obtained from a non-flat fiber resolution, whose geometry is shown in figure 22. The two non-flat surface components are identified to be

$$
S_{1} \cup S_{2}=\operatorname{gdP}_{3}\left(A_{1}+A_{1}\right) \cup \operatorname{gdP}_{8}\left(\mathrm{E}_{7}+A_{1}\right),
$$

where in addition to the gdP we also specify the Lie algebra type that the $(-2)$ curves realize, inside brackets; see appendix $\mathrm{C}$ for a discussion of the generalized del Pezzo surfaces.

This geometry is related to that of [6] (where this is referred to as $B l_{9} \mathbb{F}_{4} \cup \mathbb{F}_{0}$ ) by a number of flops between the two non-flat fiber components $S_{1}$ and $S_{2}$. 
From this geometry, it is clear that the fibral curves of the Cartan divisors of $\mathrm{E}_{8}$ and $\mathrm{SU}(2)$ are all fully contained in $S_{1} \cup S_{2}$, and form a marked subgraph of affine Dynkin diagrams in the marginal CFD. In addition there is a $(-1)$-curve $e_{2}$ on $S_{1}$, which connects the two affine nodes $F_{0}^{\mathrm{E}_{8}}$ and $F_{0}^{\mathrm{SU}(2)}$. The other $(-1)$-curve $h-e_{1}-e_{8}$ on $S_{2}$ connects the two nodes $F_{7}^{\mathrm{E}_{8}}$ and $F_{1}^{\mathrm{SU}(2)}$. There are no other curves that generate the Mori cone of $S_{1} \cup S_{2}$. Hence we conclude that the topology of the marginal CFD is the one shown in (4.15).

A subtlety here is that the rightmost marked vertex of the affine $\mathrm{SU}(2)$ in (4.15) should be interpreted as a (-1)-curve with multiplicity 2, see appendix D.2.1. In particular, applying a CFD-transition to blow down the (-1)-curve to the right of this marked curve, this multiplicity $2(-1)$-curves maps to a multiplicity two 0-curve, rather than a single $(-1)$-curve. Because of this fact, the rightmost vertex of the affine $\mathrm{SU}(2)$ can never be blown down.

The complete CFD-tree obtained by applying CFD-transitions to this marginal CFD is shown in figure 12. The number of $U(1)$ flavor symmetry factors is given by the number of unmarked vertices minus three, ${ }^{7}$ and the generators of the $\mathrm{U}(1) \mathrm{s}$ are chosen to be the white curves that do not intersect any of the green curves.

We expect the rank $Q>2$ E-string theories to have the same $6 \mathrm{~d}$ flavor symmetry and the same marginal CFD as (4.15). In particular, the tree-structure of descendants does not change - only the rank distinguishes the actual SCFTs. For instance, even though two theories have the same flavor symmetry, having a different rank means that their Coulomb branches are different, and hence must be distinguished. This is consistent with the fact that for every $Q$, there is a subset of descendants of the rank $Q$ E-string, whose Higgs branches are the $Q$-instanton of $\mathrm{E}_{n}$ [5]. That is, for every $Q$ there is a theory with superconformal flavor symmetry $\mathrm{E}_{n} \times \mathrm{SU}(2)$, which nevertheless has a different Higgs branch depending on $Q$. More generally, this applies to any case with different rank but same flavor symmetry of the marginal theory, where the $6 \mathrm{~d}$ original theories are of conformal matter type. The CFD will be the same, but the rank distinguishes the Coulomb branch of these theories.

\subsubsection{Marginal CFDs from automorphisms}

The first task in our program for the determination of all the descendant SCFTs from a given 6d SCFT is to construct the marginal theory and its associated marginal geometry. This is not always as straightforward as it may seem; there can be "outer automorphism twists" which act on the tensor branch geometry after the circle compactification, and this changes the geometry of the singular Calabi-Yau threefold realizing the marginal geometry. We discuss two rank two marginal theories that are obtained from $6 \mathrm{~d}$ in this way.

In this paper we will not construct explicit resolutions of these singular geometries to obtain the marginal theory, but instead we will directly propose the marginal CFD associated to these geometries. These two theories arise as $\mathbb{Z}_{2}$-twisted circle compactifications

\footnotetext{
${ }^{7}$ Note that we need to subtract one more than the $\left(D_{10}, I_{1}\right)$ case, because unlike the $I_{1}$ fiber there, the $I_{2}$ comes with an additional linear relation of type (2.32).
} 
of the $6 \mathrm{~d}$ theory that has a one dimensional tensor branch, an $\mathrm{SU}(3)$ gauge group, and, respectively, twelve and six fundamental hypermultipets. The CFDs are quotients of the CFD of the untwisted theory, and they have the structure of twisted affine Dynkin diagrams,${ }^{8}$ which is expected as the strongly coupled flavor symmetries predicted in [12] are $\widehat{A}_{11}^{\mathrm{tw}}$ and $\widehat{A}_{5}^{\mathrm{tw}}$.

This proposal is supported by consistency with the following known facts about these two marginal theories:

- The reducible surface $\mathcal{S}$ and the curves inside agree with the explicit collections of rational surfaces given for these theories in [6].

- The structure of the trees of the descendants of these marginal CFDs, which involves a detailed and involved structure of mass deformations, matches exactly the known trees for the descendant theories as determined in [6, 24].

We hope to turn to the systematic construction of the marginal CFDs, which involve outer automorphism twists and often involve geometries with a singular base, in future work.

3. $\mathrm{SU}(3)$ on a (-1)-curve with 12 hypermultiplets: a third starting point for rank two theories is obtained by an outer-automorphism reduction of a $6 \mathrm{~d}(1,0) \mathrm{SCFT}$, whose tensor branch is described by a (-1)-curve with an $\mathrm{SU}(3)$ gauge group and 12 fundamental hypermultiplets. We propose the marginal CFD to be

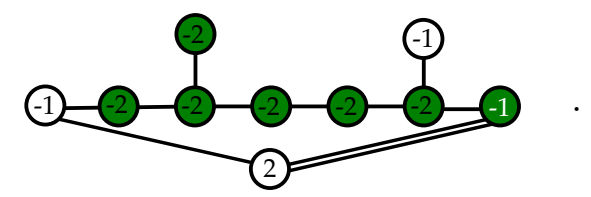

This model can be thought to arise from folding the diagram in figure 14 (b), which results in the twisted affine Dynkin diagram of $\widehat{A}_{11}^{\text {tw }} \cdot{ }^{9}$ The additional +2 curve is obtained by consistency with the CFD descendants from other starting points and the resolution geometry. This is consistent with $\mathbb{F}_{2} \cup \mathrm{dP}_{7}$ in [6]. All the descendant CFDs are shown in figure 13, where we also depict the one coming from the fourth marginal theory. At last, the number of $U(1)$ flavor symmetry factors is given by the number of unmarked vertices minus two.

4. $\mathrm{SU}(3)$ on a (-2)-curve with 6 hypermultiplets: this marginal theory is obtained by an outer-automorphism reduction of a $6 \mathrm{~d}(1,0)$ SCFT, which is given by a $(-2)$-curve

\footnotetext{
${ }^{8}$ See [87] for a nice summary of twisted affine algebras.

${ }^{9}$ Further support for obtaining this CFD from the folding of a $\widehat{D}$-type diagram is provided by a IIA construction of this $6 \mathrm{~d}$ theory. This involves a half NS5-brane stuck on top of $\mathrm{O}^{-}+11 \mathrm{D} 8$-branes. The 8-brane stack is usually responsible for the flavor symmetry, which naively would be an $S O$-type group. However, the flavor symmetry is SU(12), because of the presence of a stuck NS5. The string theory origin of this phenomenon is not yet clear [88].
} 


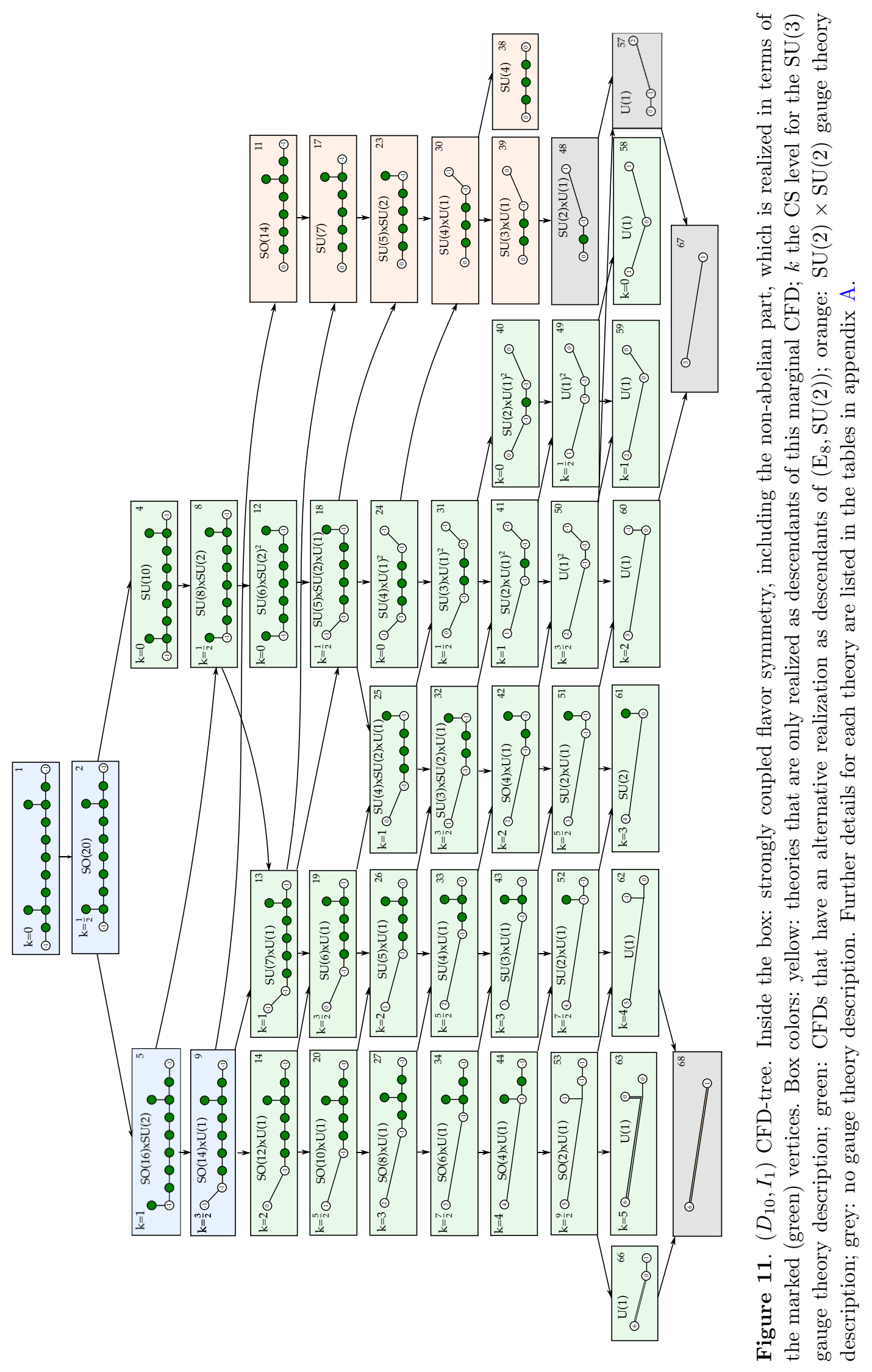




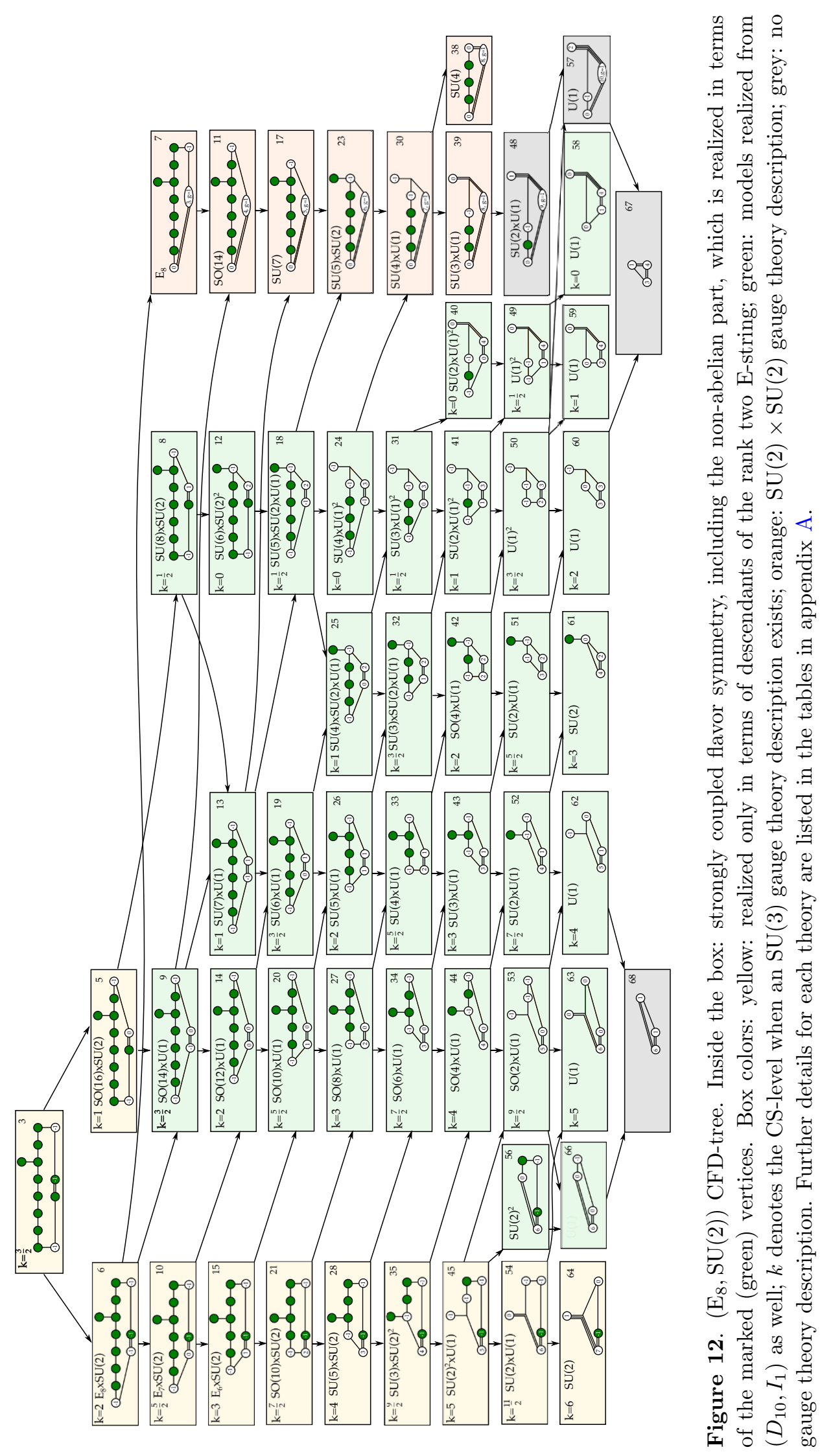




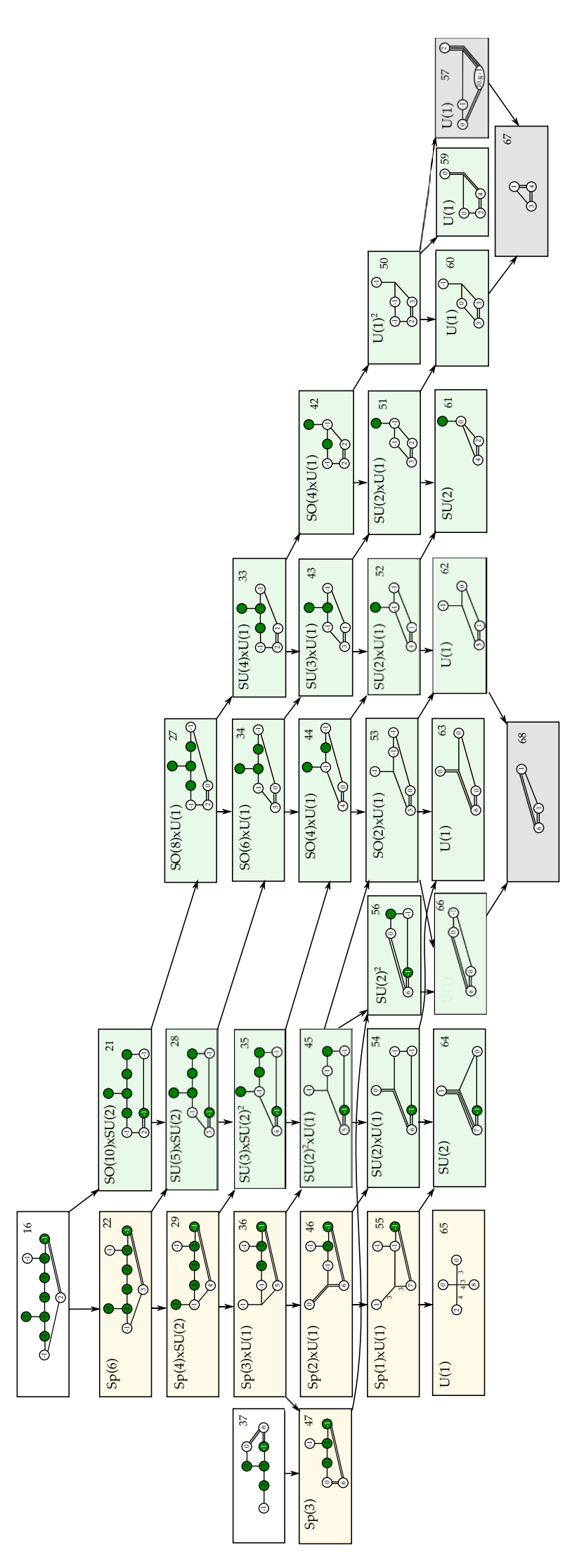

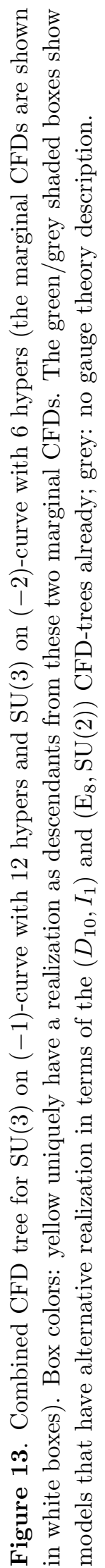




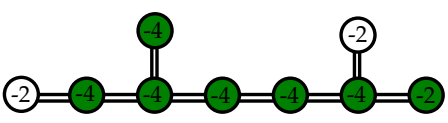

(a)

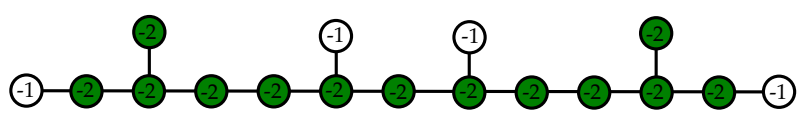

(b)

Figure 14. (a) The 'doubled' CFD of the marginal theory for SU(3) on a (-1)-curve with 12 hypermultiplets, which contains a twisted affine Dynkin diagram $\widehat{A}_{11}^{\text {tw }}$. The labels here are doubled as they are obtained by folding of (b). The unfolded version of figure (a), which contains an affine $\mathrm{SO}(24)$ Dynkin diagram.

with an $\mathrm{SU}(3)$ gauge group and 6 fundamental hypermultiplets. The marginal CFD is

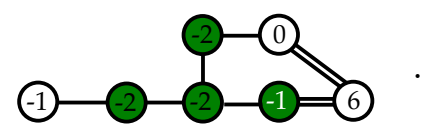

This is the geometry $\mathbb{F}_{6} \cup \mathrm{dP}_{4}$ in [6]. This case is similar to the previous one, where (4.18) is the $\mathbb{Z}_{2}$ quotient of a graph with doubled connections and selfintersection numbers, which would correspond to the fiber in the geometric realization of $\widehat{A}_{5}^{\mathrm{tw}}$. Another consistency check is that the first descendant CFD which has $\operatorname{Sp}(3)$ flavor symmetry has to match the descendant CFD from (4.17), which is model \#47 in figure 13, see also [6, 24]. All the descendant CFDs of (4.18) are included in figure 13. The number of $\mathrm{U}(1)$ flavor factors is given by the number of unmarked vertices minus two.

There are three more starting points (see appendix A), which in total give rise to a single descendant 5d SCFT according to $[6,24]$ not contained in our combined CFD-trees. This theory however requires $\mathrm{O}^{+}$planes in the dual brane-web description, and therefore is not intrinsically geometric.

\subsubsection{New flavor symmetry predictions for rank two theories}

Our methods allow us to predict all the strongly coupled flavor symmetries of the rank two descendant 5d SCFTs, whenever they have or not have an effective low-energy gauge theory description. The gauge theory descriptions for the marginal theories can be found in appendix A. The descendant gauge theories are obtained by successively decoupling flavor hypermultiplets. This procedure will form a subtree of our CFD-tree and is the topic of [31].

We present and summarize here some predictions of the so far unknown strongly coupled flavor symmetries for the following rank two gauge theories - i.e. either SU(3) or 
$\mathrm{Sp}(2)$ gauge groups:

\begin{tabular}{|c|c|c|}
\hline$\#$ & Gauge Theory & Superconformal Flavor Symmetry $G_{\mathrm{F}}$ \\
\hline 22 & $\mathrm{SU}(3)_{\frac{9}{2}}+5 \mathbf{F} ; \mathrm{Sp}(2)+2 \mathbf{A S}+3 \mathbf{F}$ & $\mathrm{Sp}(6)$ \\
29 & $\mathrm{SU}(3)_{5}+4 \mathbf{F} ; \mathrm{Sp}(2)+2 \mathbf{A S}+2 \mathbf{F}$ & $\mathrm{Sp}(4) \times \mathrm{SU}(2)$ \\
36 & $\mathrm{SU}(3)_{\frac{11}{2}}+3 \mathbf{F} ; \mathrm{Sp}(2)+2 \mathbf{A S}+1 \mathbf{F}$ & $\mathrm{Sp}(3) \times \mathrm{U}(1)$ \\
46 & $\mathrm{SU}(3)_{6}+2 \mathbf{F} ; \mathrm{Sp}(2)_{\pi}+2 \mathbf{A S}$ & $\mathrm{Sp}(2) \times \mathrm{U}(1)$ \\
55 & $\mathrm{SU}(3)_{\frac{13}{2}}+1 \mathbf{F}$ & $\mathrm{Sp}(1) \times \mathrm{U}(1)$ \\
47 & $\mathrm{Sp}(2)_{0}+2 \mathbf{A S}$ & $\mathrm{Sp}(3)$ \\
65 & $\mathrm{SU}(3)_{7}$ & $\mathrm{U}(1)$ \\
\hline
\end{tabular}

The \# refers to the numbering of the SCFTs in the tables in appendix A. Moreover, we can predict the flavor symmetries of some descendant 5d SCFTs, which only have an $\mathrm{SU}(2) \times \mathrm{SU}(2)$ quiver gauge theory description:

\begin{tabular}{|c|c|c|}
\hline$\#$ & Gauge Theory & Superconformal Flavor Symmetry $G_{\mathrm{F}}$ \\
\hline 7 & $\mathrm{SU}(2)_{0} \times[\mathrm{SU}(2)+5 \mathbf{F}]$ & $\mathrm{E}_{8}$ \\
11 & $\mathrm{SU}(2)_{0} \times[\mathrm{SU}(2)+4 \mathbf{F}]$ & $\mathrm{SO}(14)$ \\
17 & $\mathrm{SU}(2)_{0} \times[\mathrm{SU}(2)+3 \mathbf{F}]$ & $\mathrm{SU}(7)$ \\
23 & $\mathrm{SU}(2)_{0} \times[\mathrm{SU}(2)+2 \mathbf{F}]$ & $\mathrm{SU}(5) \times \mathrm{SU}(2)$ \\
30 & $\mathrm{SU}(2)_{0} \times[\mathrm{SU}(2)+1 \mathbf{F}]$ & $\mathrm{SU}(4) \times \mathrm{U}(1)$ \\
38 & $\mathrm{SU}(2)_{0} \times \mathrm{SU}(2)_{0}$ & $\mathrm{SU}(4)$ \\
39 & $\mathrm{SU}(2)_{0} \times \mathrm{SU}(2)_{\pi}$ & $\mathrm{SU}(3) \times \mathrm{U}(1)$ \\
\hline
\end{tabular}

Some of these were already anticipated using CFDs in [8].

Another interesting case is theory \#48 in figures 11 and 12 (see also the table in appendix A), for which no effective gauge theory description is known, and its SCFT flavor symmetry is $\mathrm{SU}(2) \times \mathrm{U}(1)$.

This concludes the rank two theories. Two marginal CFDs in this case have a concrete geometric realizations, which we determined using non-flat resolutions. Furthermore, the fact that the two conjectured marginal CFDs lead to descendants which perfectly fit known results including flavor symmetries lends very strong support to this approach. In particular, we find it encouraging that the tree structure generated from CFD-transitions agrees fully with the known RG-flow trees or flop trees in alternative constructions of these theories. We now turn to applying our method to the realm of the unexplored, namely to higher rank conformal matter theories and their descendant 5d SCFTs.

\section{5 $\left(\mathrm{E}_{n}, \mathrm{E}_{n}\right)$ minimal conformal matter theories}

A particularly interesting class of conformal matter theories are the exceptional $\left(\mathrm{E}_{n}, \mathrm{E}_{n}\right)$ minimal conformal matter theories for $n=6,7,8$. For $n=5$ this is the case of the rank two $\left(D_{5}, D_{5}\right)$ conformal matter that we have discussed already at length. For $n=6$ we proposed the marginal CFD and determined its descendant theories in [8], which is a rank 5 theory. The theories with $n=7$ have rank 10, and $n=8$ has rank 21 .

Here we will determine the marginal theories for all $n=6,7,8$ from a non-flat resolution sequence. We furthermore identify all descendant SCFTs and their flavor symmetries, and 


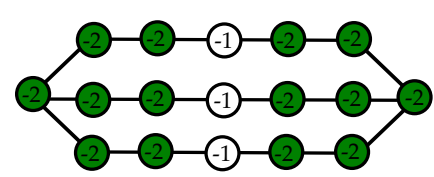

$\left(\mathrm{E}_{6}, \mathrm{E}_{6}\right)$

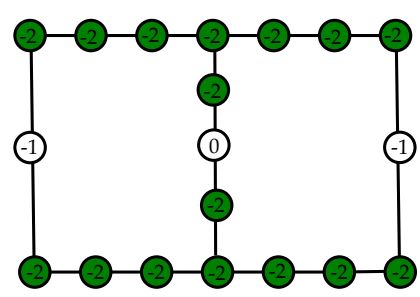

$\left(\mathrm{E}_{7}, \mathrm{E}_{7}\right)$

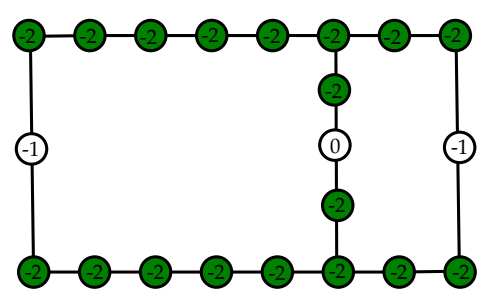

$\left(\mathrm{E}_{8}, \mathrm{E}_{8}\right)$

Figure 15. The marginal CFDs for the $\left(\mathrm{E}_{n}, \mathrm{E}_{n}\right), n=5,6,7$ minimal conformal matter theories.

match those that have a quiver gauge theory description with the associated weakly-coupled description.

The marginal CFDs for the three theories are shown in figure 15 and are derived from non-flat resolutions in appendix D.5. The descendants are obtained in the supplementary material [89]. We find

$$
\begin{array}{ll}
\left(\mathrm{E}_{6}, \mathrm{E}_{6}\right): & 93 \text { descendant SCFTs } \\
\left(\mathrm{E}_{7}, \mathrm{E}_{7}\right): & 56 \text { descendant SCFTs } \\
\left(\mathrm{E}_{8}, \mathrm{E}_{8}\right): & 127 \text { descendant SCFTs } .
\end{array}
$$

Some of these theories have a quiver description which we now discuss. However as will be clear, most of the 5d SCFTs that we found here lie outside of known weaklycoupled descriptions and it would be interesting to explore alternative descriptions to confirm our findings.

The gauge theory description for the $5 \mathrm{~d}$ marginal theory of the minimal $\left(\mathrm{E}_{7}, \mathrm{E}_{7}\right)$ conformal matter theory was conjectured in [45] to be

$$
\begin{gathered}
\mathrm{SU}(2)_{\theta=0} \\
\left.\mid{ }^{\mid}\right]-\mathrm{SU}(2)-\mathrm{SU}(3)_{k=0}-\mathrm{SU}(4)_{k=0}-\mathrm{SU}(3)_{k=0}-\mathrm{SU}(2)-[2] .
\end{gathered}
$$

By decoupling one-by one the flavor hypermultiplets, we argue that in total there are 9 different gauge theories, which fill a subtree of the descendant of the CFD. We label the descendant theories by the number of flavor on the left and right $\mathrm{SU}(2)$ or their $\theta$-angle, $\left(q_{1}, q_{2}\right)$, and we also list their superconformal flavor symmetry:

\begin{tabular}{|c|c|}
\hline$\left(q_{1}, q_{2}\right)$ & $G_{\mathrm{F}}$ \\
\hline$(2,1)$ & $\mathrm{E}_{7} \times \mathrm{E}_{7}$ \\
$(2,0) \sim(2, \pi)$ & $\mathrm{SO}(12) \times \mathrm{E}_{7}$ \\
$(1,1)$ & $\mathrm{E}_{6} \times \mathrm{E}_{6}$ \\
$(1,0) \sim(1, \pi)$ & $\mathrm{SO}(10) \times \mathrm{E}_{6}$ \\
$(0,0) \sim(\pi, \pi)$ & $\mathrm{SO}(8) \times \mathrm{E}_{6}$ \\
$(0, \pi)$ & $\mathrm{SO}(10) \times \mathrm{SO}(10)$ \\
\hline
\end{tabular}


The gauge theory description for the $5 \mathrm{~d}$ marginal theory of minimal $\left(\mathrm{E}_{8}, \mathrm{E}_{8}\right)$ conformal matter, [45], is

$$
\left.\stackrel{\mathrm{SU}(3)_{k=0}}{\mid}\right|_{k 2]-\mathrm{SU}(2)-\mathrm{SU}(3)_{k=0}-\mathrm{SU}(4)_{k=0}-\mathrm{SU}(5)_{k=0}-\mathrm{SU}(6)_{k=0}-\mathrm{SU}(4)_{k=0}-\mathrm{SU}(2)_{\theta=0}}
$$

In total there are only 3 different gauge theories descendant from this effective description of the marginal theory, and form a subtree of the full descendent CFD-tree. We again label the descendant theories by the number of flavor on the left $\mathrm{SU}(2)$ or its $\theta$-angle, $(q)$, and we also list their strongly coupled flavor symmetry:

\begin{tabular}{|c|c|}
\hline$q$ & $G_{\mathrm{F}}$ \\
\hline 1 & $\mathrm{E}_{8} \times \mathrm{E}_{8}$ \\
$0 \sim \pi$ & $\mathrm{E}_{7} \times \mathrm{E}_{8}$ \\
\hline
\end{tabular}

Our approach allows us to predict some UV dualities between theories with different theta angles on the $\mathrm{SU}(2)$ gauge nodes. This effect was already observed in simpler cases of SU-type quivers by studying the instanton operator spectrum or the superconformal index in $[34,39]$. Clearly it would be highly desirable to determine alternative quiver or gauge theory descriptions for the remaining descendants and to confirm the flavor symmetry enhancement that we see in the SCFTs. To exemplify we show the CFDs for the theories that have a quiver description in figures 16, 17 and 18. The complete list of descendants are available in the supplementary material [89].

\subsection{Higher rank: $\left(\mathrm{E}_{8}, \mathrm{SU}(n)\right)$}

In [8], we obtained an infinite sequence of 5d SCFTs which descend from the $\left(D_{k}, D_{k}\right)$ minimal conformal matter using the CFD approach (a complete CFD-tree for $k=9$ can be seen in [89]). An in depth analysis of the higher rank 5d SCFTs will appear in a future work [30].

Another class of $5 \mathrm{~d}$ SCFTs descend from $\left(\mathrm{E}_{8}, \mathrm{SU}(n)\right)$ conformal matter. These have marginal CFDs that depend on whether $n$ is even or odd, and are shown in figure 19. The derivation of the marginal CFDs from a non-flat resolution is provided in appendix D.5.

Here we present one example that we discussed already in section 3.4: the $\left(\mathrm{E}_{8}, \mathrm{SU}(3)\right)$ conformal matter. This is a rank 4 theory, and the marginal CFD can be computed from the resolution to be

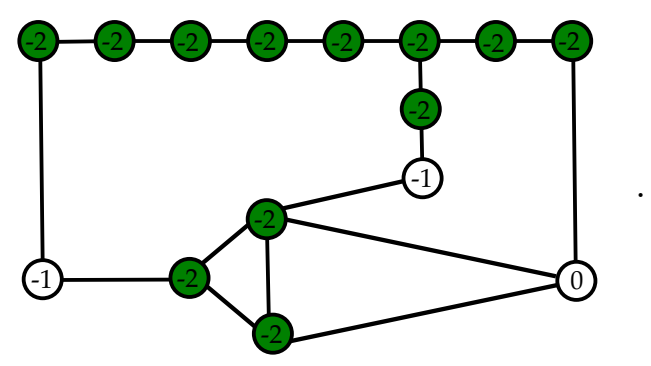




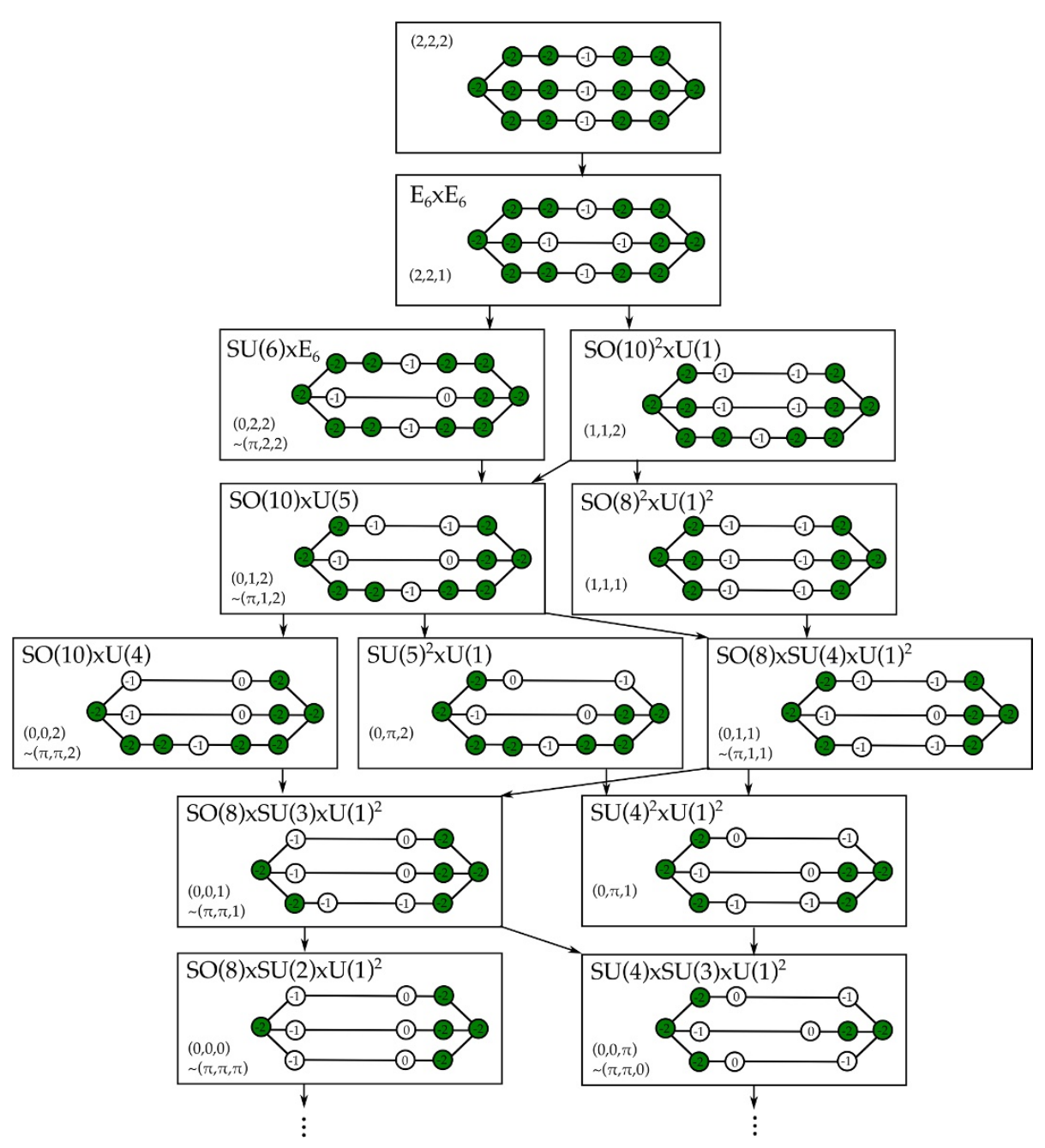

Figure 16. Subtree of the $\left(\mathrm{E}_{6}, \mathrm{E}_{6}\right)$ CFD-tree. The full CFD-tree can be seen in [89] and has 93 descendants. The theories that have a known quiver description, and comprise a small subset, are shown here.

There are 102 descendant CFDs/SCFTs. The example resolution that was constructed in section 3.4 corresponds to the CFD

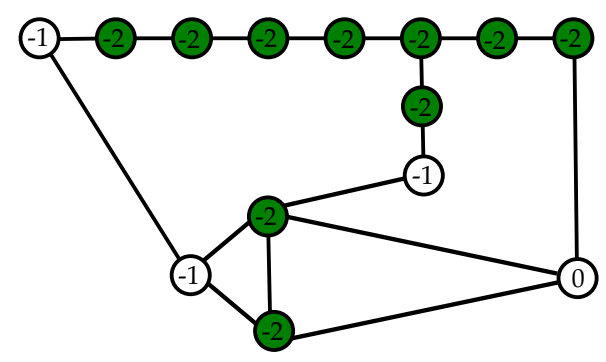

which is a descndent obtained by transitioning with the lower left (-1)-vertex in (4.26). As promised, the CFD manifestly shows the $\mathrm{E}_{7} \times \mathrm{SU}(3)$ strongly coupled flavor symmetry of the corresponding 5d SCFT. 


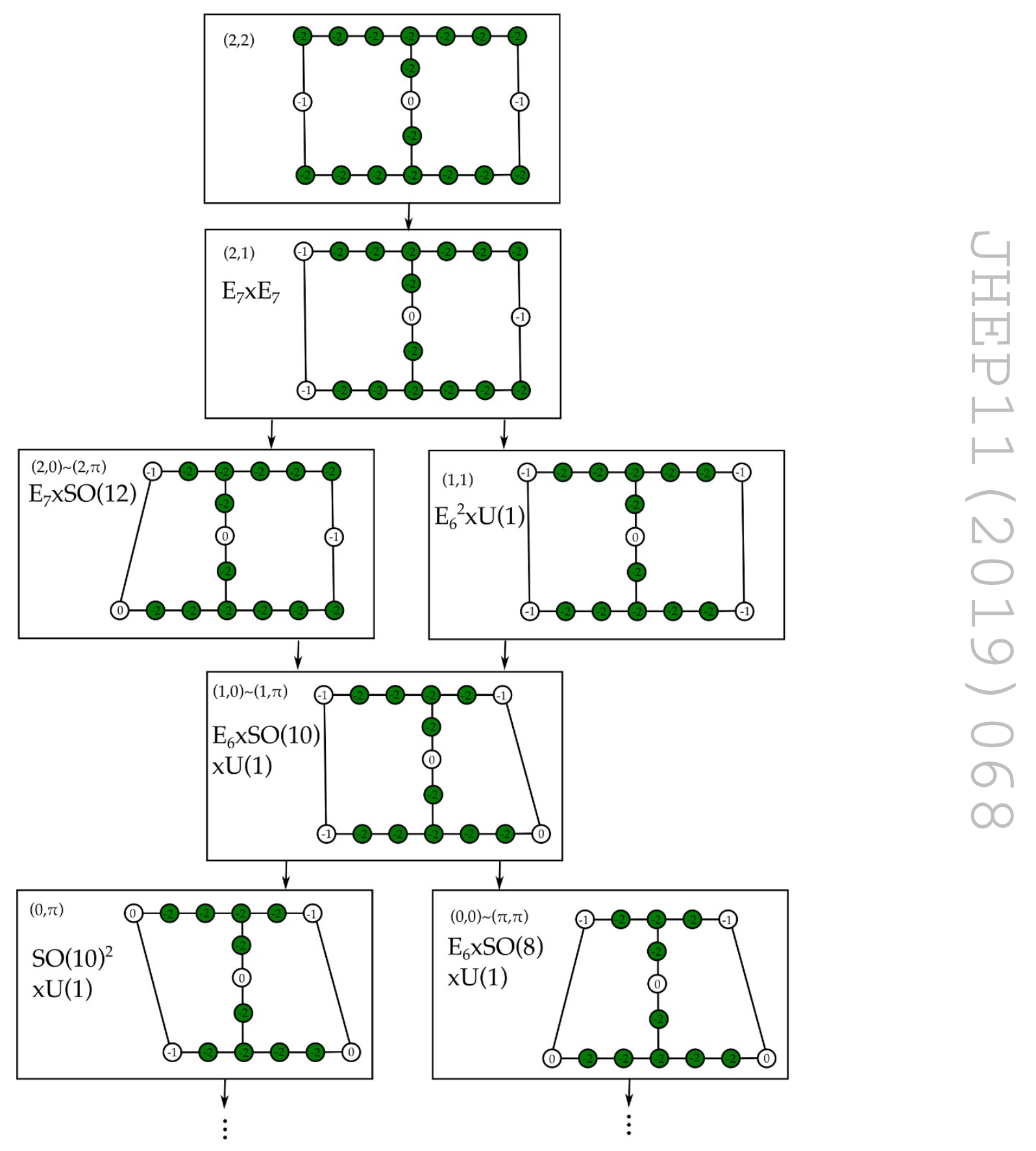

Figure 17. Subtree of the $\left(E_{7}, E_{7}\right)$ CFD-tree of theories that have a quiver description. The full CFD-tree can be seen in [89] and has 56 descendants. The theories that have a known quiver description, and comprise a small subset, are shown here. 


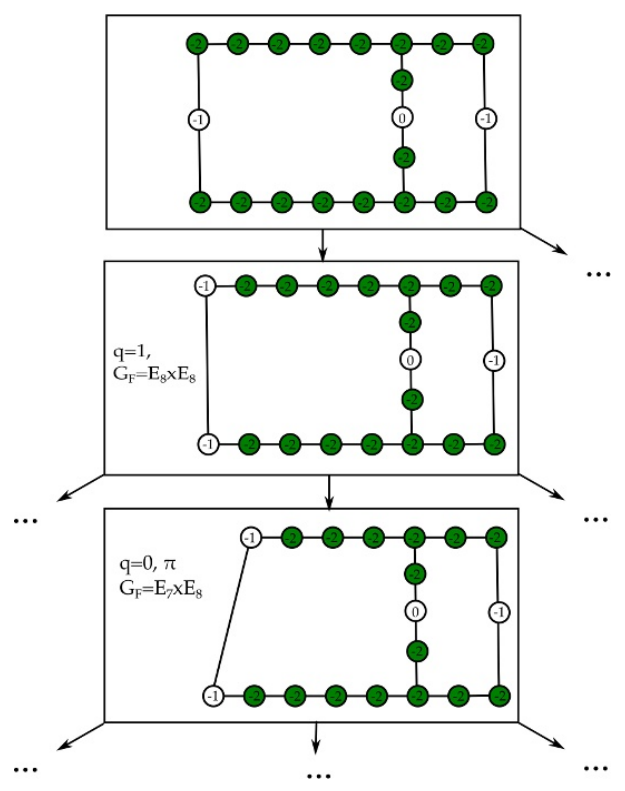

Figure 18. Subtrees of the $\left(\mathrm{E}_{8}, \mathrm{E}_{8}\right)$ CFD-tree of theories that have a quiver description, including the theta angles. The full CFD-tree can be seen in [89] and has 127 descendants. The theories that have a known quiver description, and comprise a small subset, are shown here.
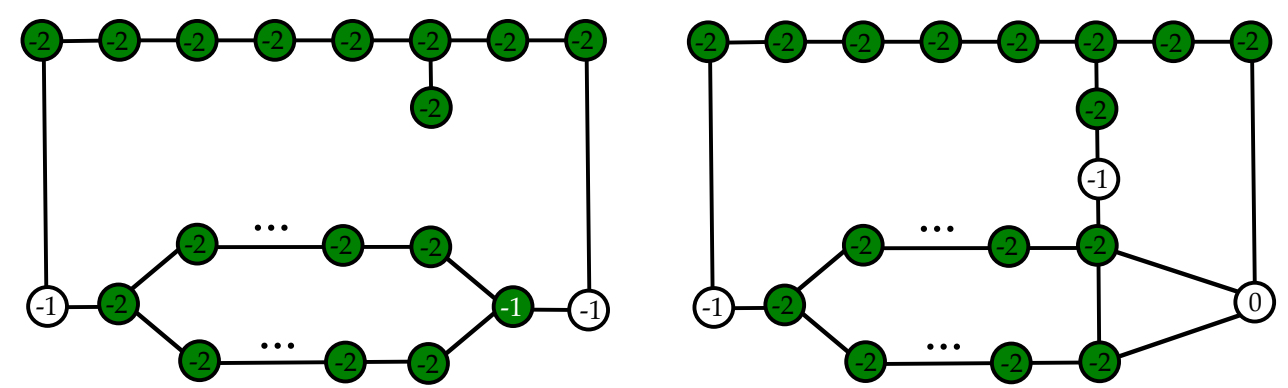

Figure 19. The marginal CFDs for $\left(\mathrm{E}_{8}, \mathrm{SU}(2 k)\right)$ and $\left(\mathrm{E}_{8}, \mathrm{SU}(2 k+1)\right)$ minimal conformal matter.

Starting with the $\left(\mathrm{E}_{8}, G_{2}\right)$ the marginal CFD is given by

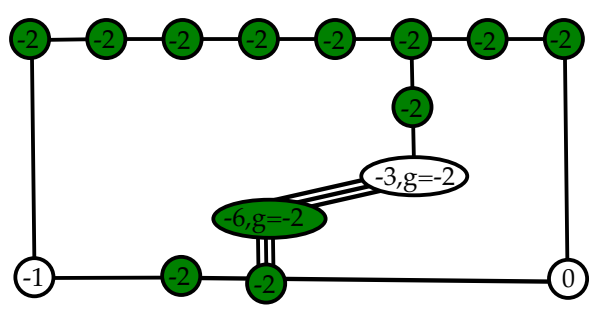

Note that the curves $(n, g)=(-6,-2)$ and $(-3,-2)$ are in fact multiplicity 3 curves of the type $(-2,0)$ and $(-1,0)$, respectively. The latter can be transitioned on, but requires removing all three curves simultaneously.

We propose a gauge theory description for this marginal theory in terms of

$$
\mathrm{SU}(2)_{\theta=0}-\mathrm{SU}(4)_{k=0}-[8] .
$$


We will explain in [31] how this gauge theory description can be derived from a ruling of the surface components in the resolved geometry. This gauge was realized as a collision in [59]. Note also that the quiver $\mathrm{SU}(2)_{\theta=0}-\mathrm{SU}(4)_{k=0}-[6]$ is an effective model, which describes one of the descendants. ${ }^{10}$ Its flavor symmetry is $\mathrm{SU}(6) \times \mathrm{SU}(3) \times \mathrm{SU}(3)[13$, $39]$, and we can see that it comes from two consecutive transitions on the $(-1)$ curves connecting one $\mathrm{SU}(3)$ node and the spcial $\mathrm{E}_{8}$ node in (4.26). At last, the total number of descendants for (4.29) is 24 , which can be obtained by successively decoupling fundamental hypermultiplets, i.e., $m_{f} \rightarrow \pm \infty$. This shifts the Chern-Simons level of SU(4), $k$, by $\pm \frac{1}{2}$. All the obtained descendant gauge theories fill a subtree of the CFDs/SCFTs tree arising from (4.26) and (4.28).

\section{$5 \quad$ BPS states}

In a $5 \mathrm{~d} \mathcal{N}=1$ theory engineered through a compactification of M-theory on a smooth Calabi-Yau threefold, which is the setup considered throughout this paper, each compact two-cycle and four-cycle contributes a BPS state. M2-branes wrapping a holomorphic 2cycle, in our case $C \subset \bigcup_{i} S_{i}=\mathcal{S}$, with the topology of a genus $g$ Riemann surface give rise to electrically charged particle states. On the other hand M5-branes wrapping four-cycles, in the case studied herein the non-flat surfaces, $S_{i}$, give rise to magnetically charged BPS string states. At the singular/SCFT limit the area of the two- and four-cycles goes to zero, and, therefore, the BPS particles become massless, and the magnetically charged string become tensionless. In what follows we will focus on the electrically charged states coming from M2-branes wrapping $C \subset \mathcal{S}$, using the general results of [71, 90, 91].

\subsection{BPS states from wrapped M2-branes}

The 8 real supercharges of the $5 \mathrm{~d} \mathcal{N}=1$ super-Poincaré algebra transform as $2 \cdot(1 / 2,0) \oplus$ $2 \cdot(0,1 / 2)$ of the $5 \mathrm{~d}$ little group $\mathrm{SO}(4)=\mathrm{SU}(2)_{L} \times \mathrm{SU}(2)_{R}$. On the M2-brane worldvolume two complex supercharges $2 \cdot(0,1 / 2)$ are broken. These two supercharges do not annihilate the ground state anymore, and they consist of 4 real fermionic degrees of freedom. In particular, by acting on a fermionic ground states, these degrees of freedom reorganize into a half-hypermultiplet

$$
H_{0}=\left(0, \frac{1}{2}\right) \oplus 2(0,0)
$$

More precisely, the BPS states are zero-modes associated to the M2-brane worldvolume theory reduced on the genus $g$ curve $C \subset \mathcal{S}$. In particular the theory has 8 fermions and 8 scalars corresponding to the directions perpendicular to the brane. The fermionic zeromodes pair with the bosonic ones, whose quantum numbers are specified by representations of the little group $\mathrm{SO}(4)=\mathrm{SU}(2)_{L} \times \mathrm{SU}(2)_{R}$. If we assume that the Riemann surface is not degenerate, the BPS states consist of two different contributions. The first one is given

\footnotetext{
${ }^{10}$ I.e., $M=11-2$, where $M=11$ is the number total number of possible mass deformation of the marginal theory for $\left(\mathrm{E}_{8}, G_{2}\right)$ conformal matter.
} 
by the zero-modes coming from the 0 -form and the 1 -form on $C$ [90],

$$
\left[\left(0, \frac{1}{2}\right) \oplus 2(0,0)\right]^{g_{C}+1}
$$

The second contribution comes from the modes associated to the deformation of $C$ inside the Calabi-Yau threefold, or, in other words, the moduli space $\mathcal{M}_{C}$. In terms of representations of the little group these states are given by

$$
\sum_{j=0}^{\frac{n}{2}} a_{j}[(j, 0)]
$$

where $n \equiv \operatorname{dim}_{\mathbb{C}}\left(\mathcal{M}_{C}\right)$, and the $a_{j}$ are gradings associated to the $\mathrm{SU}(2)_{L}$ (Lefschetz) decomposition of the cohomology groups of $\mathcal{M}_{C}$. Summing up, the total contribution for the electrically charged BPS states coming from an M2-brane wrapping curve, $C \subset \bigcup_{i} S_{i}$, of genus $g_{C}$ is [90]:

$$
\left[\left(0, \frac{1}{2}\right) \oplus 2(0,0)\right]^{g_{C}+1} \otimes\left(\sum_{j=0}^{\frac{n}{2}} a_{j}[(j, 0)]\right) .
$$

In order to compute the $a_{j}$, we need to look at the four unbroken supercharges transforming in the representation $2 \cdot(1 / 2,0)$ of $\mathrm{SO}(4)$, which act as the four differential operators $\partial, \bar{\partial}, \partial^{*}$ and $\bar{\partial}^{*}$ on the cohomology of $\mathcal{M}_{C}, H^{p, q}\left(\mathcal{M}_{C}\right)$, [71]. For example, the generator $J_{3}$ of $\mathrm{SU}(2)_{L}$ acts on a differential form $\omega$ as

$$
J_{3} \omega=\frac{\left(p+q-\operatorname{dim}_{\mathbb{C}}\left(\mathcal{M}_{C}\right)\right)}{2} \omega, \omega \in H^{p, q}\left(\mathcal{M}_{C}\right) .
$$

The differential form in $H^{p, q}\left(\mathcal{M}_{C}\right)$ will reorganize according to representation of $\mathrm{SU}(2)_{L}$, labelled by $j .{ }^{11}$ The multiplicity at each $j$ is given by the number of zero-modes, which are in a full representation of helicity $j$, i.e.

$$
a_{j}=h^{p, q}\left(\mathcal{M}_{C}\right) .
$$

We will be interested in two cases in this paper, which are the BPS states that arise from M2-branes wrapping genus zero curves with normal bundle $\mathcal{O}(-1) \oplus \mathcal{O}(-1)$ or $\mathcal{O}(0) \oplus \mathcal{O}(-2)$ inside of the Calabi-Yau threefold. These states have been analyzed by Witten [1] who showed that they are, respectively, spin 0 and spin 1 states in the sense of (5.4). In the smooth Calabi-Yau geometry, before we take the SCFT limit where the volume of the surfaces is taken to zero, the former states are hypermultiplets, whilst the latter are vector multiplets.

In order to determine the BPS states that we are interested in directly from the CFD data we will assume that each vertex, or curve, in the CFD can be written as a complete intersection between two divisors in the Calabi-Yau threefold. One of these divisors we will require to be the reducible surface, $\mathcal{S}$. As we will be interested in genus zero curves for the

\footnotetext{
${ }^{11}$ For each $j$ we will have $(2 j+1)$ values of $-j \leq \frac{\left(p+q-\operatorname{dim}_{\mathbb{C}}\left(\mathcal{M}_{C}\right)\right)}{2} \leq j$.
} 
determination of the BPS states it is necessary to first determine the genus of the linear combination of vertices. This can be computed recursively through the formula

$$
g\left(C+C^{\prime}\right)=g(C)+g\left(C^{\prime}\right)+C \cdot \mathcal{S} C^{\prime}-1 .
$$

Using the Calabi-Yau condition together with the complete intersection property one can see that the normal bundle to any genus zero curve, $C$, that is a non-negative linear combination of vertices in the CFD, can be written as

$$
N_{C / Y}=\mathcal{O}(C \cdot \mathcal{S} C) \oplus \mathcal{O}(-2-C \cdot \mathcal{S} C) .
$$

Thus we can see that spin 0 states, which come from M2-branes wrapping curves that have normal bundle $\mathcal{O}(-1) \oplus \mathcal{O}(-1)$ inside the Calabi-Yau threefold come from $C$ s such that $C \cdot \mathcal{S} C=-1$, such $C$ will be referred to as $(-1)$-curves. The spin 1 states, which come from curves with normal bundle $\mathcal{O}(0) \oplus \mathcal{O}(-2)$ have two origins, either $C \cdot \mathcal{S} C=-2$ or $C \cdot \mathcal{S} C=0$. We will not consider the former as they give rise to "decoupled states" [74, 92] that decouple in the SCFT limit, and moreover, the partition function including such states do not preserve the $G_{\mathrm{F}}$ flavor symmetry [92]. The latter, which we refer to as (0)-curves give rise to the spin 1 BPS states of the SCFT.

For the representation of such states under the superconformal flavor symmetry $G_{\mathrm{F}}$, one merely needs to compute the intersection numbers $C \cdot D_{i}$, where $D_{i}$ s are various divisors that generate the non-abelian and abelian parts of $G_{\mathrm{F}}$. In the language of CFD, these numbers can be computed from the intersection relation of the nodes that correspond to $C$ and $D_{i}$.

To get the highest weight state for a representation of the non-abelian part, we require that such a curve $C$ does not intersect negatively with any $D_{i}$ that generates the nonabelian flavor symmetry (equivalently, the fully wrapped Cartan nodes, or flavor curves, $F_{i}$ in the CFD). We will list all the genus zero curves generating spin 0 and spin 1 BPS states for all the $5 \mathrm{~d}$ SCFTs considered in this paper.

In conclusion, the strategy for determining a part ${ }^{12}$ of the BPS spectrum of the SCFT is as follows. We consider a curve $C$ formed as a non-negative linear combination of vertices of the CFD (associated to the SCFT of interest). There are then two cases in which we are interested

$$
\begin{array}{lll}
g(C)=0 \text { and } C \cdot \mathcal{S} C=-1 & \Rightarrow & \text { Spin } 0 \text { BPS state } \\
g(C)=0 \text { and } C \cdot \mathcal{S} C=0 & \Rightarrow & \text { Spin } 1 \text { BPS state }
\end{array}
$$

The former are referred to as (-1)-curves, and the latter as (0)-curves. Each such curve $C$ is associated to a weight of the superconformal flavor symmetry by considering the intersection numbers $C \cdot \mathcal{S} F_{i}$, where, again, the $F_{i}$ are the marked vertices in the CFD. The collection of all such curves, $C$, which give rise of BPS states of the same spin, and with their corresponding weights form themselves into full representations of the superconformal flavor

\footnotetext{
${ }^{12}$ Throughout this paper we will generally state that we are "determining the BPS states" of a particular SCFT. By this we will refer only to those BPS states that are spin 0 or spin 1 and arise from M2-branes wrapping genus zero curves with self-intersection either 0 or -1 , as described in this paragraph.
} 
symmetry. To determine the appearing representations it is enough to determine the curves, satisfying the above conditions, that are associated to weights within the fundamental Weyl chamber of the flavor algebra; in short, we need to obtain the $C$ associated to spin 0 and spin 1 , as above, which further satisfy

$$
C \cdot \mathcal{S} F_{i} \geq 0
$$

\subsection{BPS states of rank one SCFTs}

We begin by determining the BPS states associated to the ten rank one interacting $5 \mathrm{~d}$ $\mathcal{N}=1$ SCFTs, for which the CFDs were written down in figure 10. The BPS states are summarized in table 4. The Lie group representations follow the conventions of [93]. The $\mathrm{U}(1)$ charges are written in the subscripts unless the flavor symmetry group $G_{\mathrm{F}}$ is entirely abelian. The spin 0 and 1 spectrum from the genus 0 curves matches the results in [94], which are derived using orthogonal methods.

As a representative example we will consider the rank one theory with superconformal flavor symmetry group $\mathrm{E}_{8}$, which is associated to the CFD

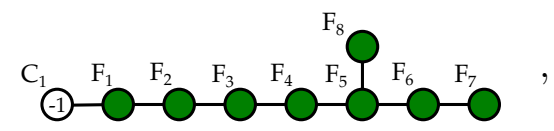

where we have introduced the labels $C_{1}$ and $F_{i}$ for the, respectively unmarked and marked, vertices. There is a single $(-1)$-curve inside of the fundamental Weyl chamber

$$
C^{\text {spin } 0}=C_{1}
$$

which is associated to the weight $(1,0,0,0,0,0,0,0)$ of the $\mathrm{E}_{8}$ flavor group. This is the highest weight of the $\mathbf{2 4 8}$ representation of $\mathrm{E}_{8}$.

Similarly, the only (0)-curve that corresponds to a highest weight vector is

$$
C^{\text {spin } 1}=2 C_{1}+2 F_{1}+2 F_{2}+2 F_{3}+2 F_{4}+2 F_{5}+F_{6}+F_{8}
$$

which corresponds to the weight vector $(0,0,0,0,0,0,1,0)$ of $\mathrm{E}_{8}$. The M2-brane wrapping mode over such curve gives rise to a spin 1 state in the representation $\mathbf{3 8 7 5}$.

We summarize in table 4 our results for all rank one theories. In particular we present the CFDs, the weakly-coupled gauge theory description, the strongly coupled flavor symmetry and the spin 0 and 1 BPS states coming from $g_{C}=0$ holomorphic curves.

\subsection{BPS states of rank two SCFTs}

In this section we consider some representative examples of the application of the above given method for the determination of the BPS spectra in the case of the rank two $5 \mathrm{~d}$ SCFTs. We list all of the rank two SCFTs with their CFD, flavor symmetry, gauge theory descriptions and BPS states in the tables in appendix A.1.2. 


\begin{tabular}{|c|c|c|}
\hline curve $C$ & $\left(C \cdot F_{i}\right),(i=1, \ldots, 9)$ & Rep. \\
\hline$C_{1}$ & $(1,0,0,0,0,0,0,0,0)$ & $(\mathbf{2 4 8}, \mathbf{1})$ \\
$C_{2}$ & $(0,0,0,0,0,0,0,0,2)$ & $(\mathbf{1}, \mathbf{3})$ \\
$C_{3}$ & $(0,0,0,0,0,0,1,0,1)$ & $(\mathbf{3 8 7 5}, \mathbf{2})$ \\
\hline
\end{tabular}

Table 2. The rational (-1)-curves giving rise to spin 0 BPS states on the CFD of the SCFT with $G_{\mathrm{F}}=\mathrm{E}_{8} \times \mathrm{SU}(2)$.

Consider first the following illustrative example, where we analyze the BPS states from the CFD (4.4). We label the nodes as below:

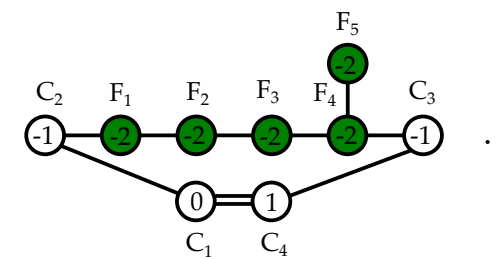

The rational ( -2$)$-curves $F_{1}, \cdots, F_{5}$ generate the $\mathrm{SU}(6)$ flavor symmetry and the (0)-curve $C_{1}$ generates the $\mathrm{U}(1)$ flavor symmetry. The rational (-1)-curves in (5.14) are $C_{2}$ and $C_{3}$, which correspond to the highest weight vector of the representation $\mathbf{6}$ and $\overline{\mathbf{1 5}}$ of SU(6) respectively. Their $\mathrm{U}(1)$ charges can be read off from

$$
C_{2} \cdot C_{1}=1, C_{3} \cdot C_{1}=0 .
$$

Hence the M2-brane wrapping modes give rise to $5 \mathrm{~d}$ hypermultiplets in the representations $\mathbf{6}_{1}$ and $\overline{\mathbf{1 5}}_{0}$. The rational (0)-curves are $C_{1}, C_{2}+F_{1}+F_{2}+F_{3}+F_{4}+C_{3}$ and $2 C_{3}+2 F_{4}+F_{3}+F_{5}$, which are in the representations $\mathbf{1}_{0}, \overline{\mathbf{6}}_{1}$ and $\mathbf{1 5}_{0}$ of $\mathrm{SU}(6) \times \mathrm{U}(1)$ respectively, which gives rise to the spin 1 BPS spectrum.

We also analyze one of the more complicated examples here, which is the 5D SCFT with flavor symmetry $G_{\mathrm{F}}=\mathrm{E}_{8} \times \mathrm{SU}(2)$. We label the nodes in the CFD as follows:

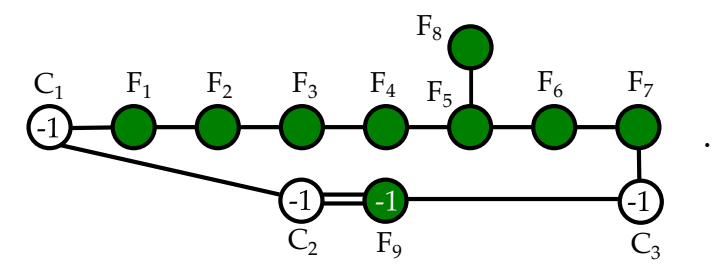

Notice that the Cartan node $F_{9}$ is actually a $(-1)$-curve with weight 2 , which is then effectively fully wrapped and contributes to the non-abelian flavor symmetry. We list the genus- 0 curves contributing to the spin-0/1 BPS spectrum in table 2 and table 3 respectively, with their genus, self-intersection number, intersection numbers with the Cartan nodes $\left(F_{1}, \ldots, F_{9}\right)$ and the representation under $G_{\mathrm{F}}=\mathrm{E}_{8} \times \mathrm{SU}(2)$.

As the final example, we discuss the case of the 5D SCFT with flavor symmetry $G_{\mathrm{F}}=\operatorname{Sp}(6)$, with the following CFD:

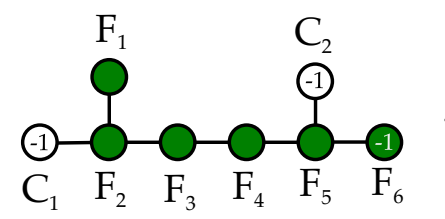




\begin{tabular}{|c|c|c|}
\hline curve $C$ & $\left(C \cdot F_{i}\right),(i=1, \ldots, 9)$ & Rep. \\
\hline$C_{1}+C_{2}$ & $(1,0,0,0,0,0,0,0,2)$ & $(\mathbf{2 4 8}, \mathbf{3})$ \\
$2 F_{1}+2 F_{2}+2 F_{3}+2 F_{4}+2 F_{5}+2 F_{6}+F_{8}+2 C_{1}$ & $(0,0,0,0,0,0,1,0,0)$ & $(\mathbf{3 8 7 5}, \mathbf{1})$ \\
$F_{2}+2 F_{3}+3 F_{4}+4 F_{5}+3 F_{6}+2 F_{7}+2 F_{8}+C_{1}+C_{3}$ & $(2,0,0,0,0,0,0,0,1)$ & $(\mathbf{2 7 0 0 0 , 2})$ \\
$F_{1}+F_{2}+F_{3}+F_{4}+F_{5}+F_{6}+F_{7}+C_{1}+C_{3}$ & $(0,0,0,0,0,0,0,1,1)$ & $(\mathbf{1 4 7 2 5 0 , 2})$ \\
$F_{4}+2 F_{5}+2 F_{6}+2 F_{7}+2 F_{8}+2 C_{3}$ & $(0,0,1,0,0,0,0,0,2)$ & $(\mathbf{2 4 5 0 2 4 0 , 3})$ \\
\hline
\end{tabular}

Table 3. The rational (0)-curves giving rise to spin 1 BPS states on the CFD of the SCFT with $G_{\mathrm{F}}=\mathrm{E}_{8} \times \mathrm{SU}(2)$.

Note that the right most Cartan node (long node) of the $\mathrm{Sp}(6)$ is in fact a (-1)-curve with multiplicity 2 , and the intersection number between $F_{6}$ and $F_{5}$ should be 1 if $F_{6}$ is interpreted to be a (-1)-curve. Only when this is true, can we match the CFDs from the starting point $\left(\mathrm{E}_{8}, \mathrm{SU}(2)\right)$ and the Model 3, see the 5D SCFT with $G_{\mathrm{F}}=\mathrm{SO}(10) \times \mathrm{SU}(2)$ and $\mathrm{SU}(3)_{7 / 2}+5 \mathbf{F}$ gauge theory description for example.

Then the curves giving rise to spin 0 BPS states are the rational curves $C_{1}$ and $C_{2}$, which are the highest weight vector of the representations 65 and $\mathbf{5 7 2}$ respectively. The curves giving rise to spin-1 BPS states are the rational curves $F_{1}+2 F_{2}+F_{3}+2 C_{1}$ and $C_{1}+F_{2}+F_{3}+F_{4}+F_{5}+F_{6}+C_{2}$, which give rise to 429 and $\mathbf{4 5 7 6}$ representations respectively.

\section{Conclusions and outlook}

In this work we have put forward a description of $5 \mathrm{~d} \mathcal{N}=1$ SCFTs in terms of graphs that encodes relevant mass deformations, superconformal flavor symmetries and certain BPS states. The underlying structures are founded upon the realization of 5d SCFTs in M-theory on elliptic Calabi-Yau threefolds $Y$ with non-minimal singularities. Associated to an elliptic fibration, there is a $6 \mathrm{~d} \mathcal{N}=(1,0)$ SCFT (which is obtained by compactifying Ftheory on $Y$ ), whose circle reduction including holonomies in the flavor symmetry yields $5 \mathrm{~d}$ SCFTs on their Coulomb branch. Geometrically, the latter is described by topologically distinct configurations of compact surfaces $\mathcal{S}=\bigcup_{j} S_{j}$ and ruled non-compact divisors $F_{i} \hookrightarrow D_{i}$ that resolve the non-minimal singularity. The limit where $\mathcal{S}$ collapses to a point - which by construction exists as a partial resolution of the singularity - corresponds to the origin of the Coulomb branch, where the strongly coupled SCFT lives.

While there can be in general many birationally equivalent geometries that realize the same 5d SCFT in this fashion, the key aspect of our approach is to manifestly keep track of the flavor symmetries. This is achieved by tracking those fibral curves $F_{i}$ of the noncompact divisors $D_{i}$ that are contained within $\mathcal{S}$. In a given resolution geometry, these so-called flavor curves intersect in the Dynkin diagram of the $5 \mathrm{~d}$ superconformal flavor group $G_{\mathrm{F}}$. It is these flavor curves, together with additional curves inside $\mathcal{S}$ describing possible mass deformations, that are encoded in the CFDs and provide a succinct characterization of each $5 \mathrm{~d}$ SCFT. In section 4, we have associated to every (equivalence class of) resolution(s) a CFD. Linear combinations of the vertices inside a CFD correspond to a chain of holomorphic curves, whose mutual intersections are indicated by edges of the graph. This information furthermore allows us to efficiently determine spin 0 and 1 BPS 
states from M2-branes wrapping rational curves in $\mathcal{S}$, see section 5. Moreover, any such graph depicts the flavor curves specifying the strongly coupled flavor symmetry $G_{\mathrm{F}}$, as well as possible mass deformations of the corresponding 5d SCFT. Such deformations correspond to transitions between CFDs, which are encoded in simple combinatorial rules.

This sets up an elegant way to classify all $5 \mathrm{~d}$ SCFTs originating from a given $6 \mathrm{~d}$ SCFT via circle reduction. Starting from the CFD of the unique 5d marginal theory associated with the 6d SCFT, the graph transitions generate a tree of descendant 5d SCFTs including information about their superconformal flavor symmetry and spin 0 and 1 BPS states. At rank one and two, this graph-based classification agrees perfectly with known results [6, 24] and furthermore adds the flavor symmetry and BPS states for each theory. The full list of rank one and rank two theories are in the tables in appendix A.2, and the CFD-trees showing the transitions are in figures 10 for rank one, and in figures 11, 12, and 13 for rank two.

A major advantage of this approach is that it easily generalizes to higher rank, once the CFD for the marginal theory is computed. We determine the marginal CFDs and their descendants of $\left(\mathrm{E}_{n}, \mathrm{E}_{n}\right),\left(D_{k}, D_{k}\right)$, and $\left(\mathrm{E}_{8}, \mathrm{SU}(n)\right)$ conformal matter theories, providing predictions for previously unknown superconformal field theories and flavor enhancements.

At the technical level, the intuition for our proposal is largely based on so-called nonflat resolutions of non-minimal elliptic singularities. In these blow-ups, the smooth space is still elliptically fibered over the same base $B$, but has the compact surfaces $S_{j}$ inserted into a special fiber in codimension two, resulting in a non-flat fibration. However, as emphasized by the rank two examples in section 4.4, our graph-based approach is in no way limited to non-flat resolutions, and only requires tracking the flavor curves that are contained in the compact divisors in the geometry. Indeed, the description in terms of CFDs is applicable both to non-flat resolutions and the tensor branch geometries. Resolutions that are a combination of base and fiber resolutions are also useful in determining the marginal theories for certain higher rank cases. All that our proposal requires is a thorough understanding of the marginal geometry, i.e., a resolution where all codimension one fibers $F_{i}$ are contained in $S_{j}$.

The complementary approach in the companion paper Part II [31] is to determine the SCFT-relevant information from the effective gauge theory descriptions of the marginal theory. There, we derive the CFDs entirely from the analysis of the Coulomb branch of 5 d SCFTs, including the gauge theory descriptions of the descendant theories - whenever such descriptions exist. This gives another, independent check of our proposal.

A complete classification program will require determining the marginal CFDs for all $6 \mathrm{~d}$ SCFTs and we return to this in Part III [30]. An obvious generalization includes combining CFDs and formulating rules for gluing marginal CFDs by gauging common subgroups of the flavor symmetry. These are gluing operations on CFDs for instance of the type

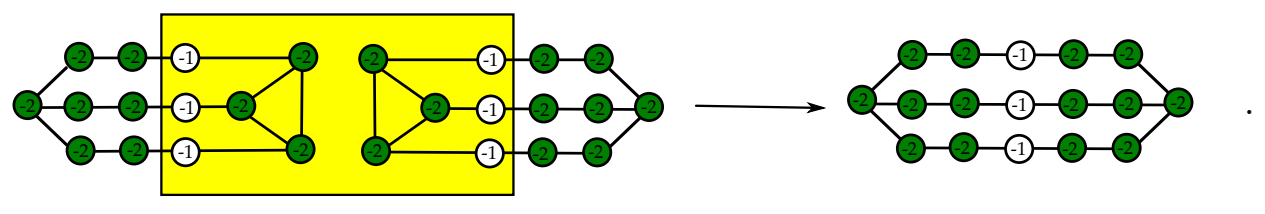


This combines two $\left(\mathrm{E}_{6}, \mathrm{SU}(3)\right)$ rank one conformal matter theories by gauging a common SU(3) flavor symmetry. Geometrically, this corresponds to compactifying the surface components, where the $\mathrm{SU}(3) \mathrm{s}$ are realized. The $(-1)$-curve in the $\left(\mathrm{E}_{6}, \mathrm{E}_{6}\right)$ marginal CFD can be interpreted as a combination of three curves $(-1) /(-3) /(-1)$ from the tensor branch resolution of $\left(\mathrm{E}_{6}, \mathrm{E}_{6}\right)$ non-minimal Weierstrass model, and there are three of them due to the $S_{3}$ symmetry of the affine $\mathrm{E}_{6}$ and $A_{2}$ Dynkin diagram. After this gauging the resulting CFD is that of the $\left(\mathrm{E}_{6}, \mathrm{E}_{6}\right)$ conformal matter theory.

The main hallmark of our approach is that the superconformal flavor symmetries are manifest in the description of 5d SCFTs. Equally, we have seen that certain BPS states associated to genus zero curves can be easily read off from the CFD. Developing the enumeration of more general BPS states, using this description in terms of CFDs, is something we believe is worthwhile exploring. It may be useful to connect this to the approach in $[95,96] .^{13}$

\section{Acknowledgements}

We thank H. Hayashi, J. J. Heckman, N. Mekareeya, D. Morrison, W. Taylor and M. Weidner for discussions. The work of FA, SSN, YNW is supported by the ERC Consolidator Grant number 682608 "Higgs bundles: supersymmetric Gauge Theories and Geometry (HIGGSBNDL)". CL is supported by NSF CAREER grant PHY-1756996. LL is supported by DOE Award DE-SC0013528Y. YNW thanks the Aspen Center for Physics, and support in part by the Simons Foundation. CL and FA acknowledge the Pollica Workshop 2019 for hospitality. We thank the String-Phenomenology and String-Math 2019 conferences, where this work was presented.

\section{A Summary for rank one and two 5d SCFTs}

This appendix serves two purposes: we first list all rank one and two marginal $5 \mathrm{~d}$ theories and their geometric, CFD, and gauge theoretic realization. In appendix A.2 we summarize all descendant 5d SCFTs in rank one and two. These are a detailed description of the theories already shown in the CFD-trees in section 4.

\section{A.1 Marginal theories in 5d}

Marginal theories are $5 \mathrm{~d}$ theories obtained from $6 \mathrm{~d}$ SCFTs by circle-reduction. They do not have UV completion in $5 \mathrm{~d}$, but flow to a fixed point in $6 \mathrm{~d}$. In terms of classifications of $5 \mathrm{~d}$ SCFTs, they are the starting points of our CFD-trees. In this section, we summarize the marginal theories of rank one and two, combining all the data that goes into the CFDdescendant computation. In addition we also supplement the gauge theoretic description for the marginal theories, which are the topic of the companion paper [31].

To characterize the $6 \mathrm{~d}$ theories relevant for rank one and two, it is useful to list their tensor branch geometries, which are essentially the resolved base of the F-theory realization. We use standard notation conventions: non-minimal points in the base of the F-theory elliptic Calabi-Yau threefold are blowun up by inserting a chain of $\mathbb{P}^{1} \mathrm{~s}$, until there are no

\footnotetext{
${ }^{13}$ We thank D. Gaiotto and P. Longhi for bringing this to our attention.
} 
more non-minimal singularities. On a curve $\Sigma$ with $\Sigma^{2}=-n$, the elliptic fiber can still have a singularity, of type $\mathfrak{g}$, and we denote this by

$$
\stackrel{\mathfrak{g}}{n} .
$$

Over non-compact curves the fiber can also be singular, which corresponds to flavor symmetries of the tensor branch theory, and we distinguish this case by denoting these in square brackets, as usual

$$
[\mathfrak{g}] .
$$

\section{A.1.1 Marginal theories for rank one SCFTs}

The marginal theory, from which the rank one 5d SCFTs descend, is the 6d rank one E-string theory, whose tensor branch is

$$
\left[\mathfrak{e}_{8}\right]-1,
$$

\begin{tabular}{|c|c|c|}
\hline Marginal CFD & Gauge Theory & Box Graph \\
\hline & $\mathrm{SU}(2)+8 \mathbf{F}$ & $\square 111 \square+110$ \\
\hline
\end{tabular}

i.e., this has an $\mathrm{E}_{8}$ flavor symmety and one compact self-intersection -1 curve in the base. The summary table for the rank one case is:

This table describes all the data for the marginal theory in 5d: the CFD (from which we determine all descendant 5d SCFTs by CFD-transitions), the weakly-coupled gauge theory description in terms of an $\mathrm{SU}(2)$ gauge theory with 8 fundamental flavors, and the box graph, which gives a simple graphical characterization of the Coulomb branch phase, and will be part of the compantion paper [31].

\section{A.1.2 Marginal theories for rank two SCFTs}

There are several 6d SCFTs, which upon circle compactification and, in some cases, outer automorphism twists, give rise to rank two $5 \mathrm{~d}$ theories $[6,12]$. We list these here, as well as the data of the CFDs and box graphs that are key to our classification approach:

1. Rank two E-string: the tensor branch for this theory is

$$
\left[\mathfrak{e}_{8}\right]-1-2-\left[\mathfrak{s u}_{2}\right] .
$$

\begin{tabular}{|c|c|c|}
\hline Marginal CFD & Gauge Theory & Box Graphs \\
\hline & $\mathrm{SU}(3)_{\frac{3}{2}}+9 \mathbf{F}$ & \\
\hline & $\mathrm{Sp}(2)+8 \mathbf{F}+1 \mathbf{A} \mathbf{S}$ & 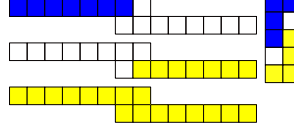 \\
\hline & {$[2 \mathbf{F}+\mathrm{SU}(2)] \times[\mathrm{SU}(2)+5 \mathbf{F}]$} & 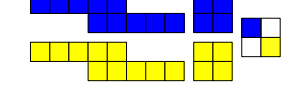 \\
\hline
\end{tabular}

The summary table for the marginal theory is as follows: 
Again the box graph description for each of the weakly-coupled gauge theory descriptions is added for ease of comparison of this paper and Part II [31]. The above table lists the Chern-Simons level for the SU groups.

2. $\left(D_{5}, D_{5}\right)$ minimal conformal matter theory: this $6 \mathrm{~d}$ SCFT, or alternatively $\left(D_{10}, I 1\right)$, has flavor symmetry $\mathrm{SO}(20)$. The tensor branch geometry is

$$
\left[\mathfrak{s o}_{10}\right]-\stackrel{\mathfrak{s p}}{1}^{1}-\left[\mathfrak{s o}_{10}\right] .
$$

The summary table for the marginal theory is as follows:

\begin{tabular}{|c|c|c|}
\hline Marginal CFD & Gauge Theory & Box Graphs \\
\hline \multirow{3}{*}{ (1)-0-0-0-0-0-0-0-0 } & $\mathrm{SU}(3)_{0}+10 \mathbf{F}$ & \\
\hline & $\operatorname{Sp}(2)+10 \mathbf{F}$ & 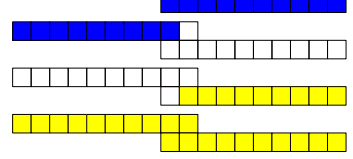 \\
\hline & {$[4 \mathbf{F}+\mathrm{SU}(2)] \times[\mathrm{SU}(2)+4 \mathbf{F}]$} & 410 \\
\hline
\end{tabular}

3. $\mathrm{SU}(3)$ on a $(-1)$ curve with 12 hypers: this theory has in fact one hypermultiplet in the antisymmetric representation of $\mathrm{SU}(3)$ and 11 hypers in the fundamental representation. The flavor symmetry at the superconformal point is $\mathrm{SU}(12)$, and the tensor branch geometry is

$$
[1 \mathbf{A S}] / / \stackrel{\mathfrak{s u}_{3}}{1}-[11],
$$

where // indicates that a non-compact curve intersect tangentially the -1 curve, and $1 \mathrm{AS}$ refers to one hypermultiplet in the antisymmetric representation of the gauge group. Upon $S^{1}$ compactification with a $\mathbb{Z}_{2}$ automorphism twist we obtain a rank two theory that is the marginal theory with the following data:

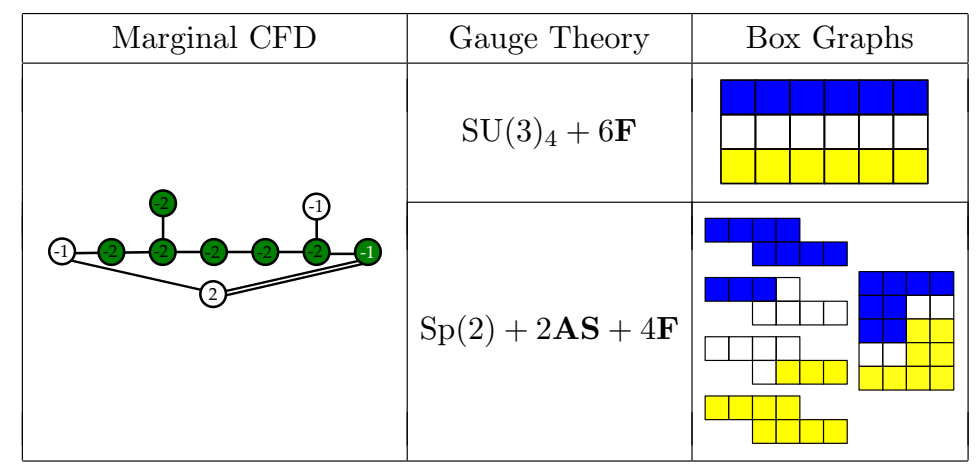

There is also a $G_{2}+6 \mathbf{F}$ gauge theory description and we will expand on this in [31]. 
4. $\mathrm{SU}(3)$ theory on a $(-2)$-curve with 6 hypers: this $\mathrm{SU}(3)$ theory with 6 fundamental hypers has $6 \mathrm{~d}$ superconformal flavor symmetry $\mathrm{SU}(6)$. Its tensor branch is

$$
\left[\mathfrak{s u}_{3}\right]-\stackrel{\mathfrak{s u}_{3}}{2}-\left[\mathfrak{s u}_{3}\right] .
$$

Applying a $\mathbb{Z}_{2}$-automorphism reduces this to a $5 \mathrm{~d}$ marginal theory of rank two. The summary data is as follows:

\begin{tabular}{|c|c|c|}
\hline Marginal CFD & Gauge Theory & Box Graphs \\
\hline & $\operatorname{Sp}(2)_{0}+3 \mathbf{A} \mathbf{S}$ & \\
\hline & & \\
\hline
\end{tabular}

In addition to these four marginal theories, there are two, which which have gauge theoretic descriptions, but a less clear geometric description in all the marginal theory phases [6]. In terms of the rank two classification [6, 24], they give rise to one single extra theory that are not obtained otherwise as descendants of the above four marginal theories (a theory descending from a model with an $\mathrm{O}^{+}$-plane, which is not purely geometric).

5. The $A_{4}(2,0) 6 \mathrm{~d}$ theory has a tensor branch of the form

$$
2-2-2 .
$$

The marginal geometry is given by three surfaces, whose geometry is $T^{2} \times \mathbb{P}^{1}$, and which are glued along the $T^{2}$. The spectrum of the marginal theory apart from the vector multiplet has an additional adjoint scalar due to the $g=1$ curve as base of the ruling [5]. This leads to the $5 \mathrm{~d} \mathrm{SU}(4) \mathcal{N}=2$ gauge theory, which consists of a $\mathcal{N}=1$ vector multiplet and a real scalar in the adjoint of $\mathrm{SU}(4)$. If we turn on a $\mathbb{Z}_{2}$-automorphism twist the $5 \mathrm{~d}$ marginal theory is specified by

$$
\begin{array}{|c|}
\hline \text { Gauge Theory } \\
\hline \mathrm{Sp}(2)_{\pi}+1 \mathbf{A d j} \\
\hline \mathrm{SU}(3)_{\frac{3}{2}}+1 \mathbf{S y m} \\
\hline
\end{array}
$$

where 1Sym means one hypermultiplet in the symmetric representation of the gauge group.

The duality between the $\mathrm{SU}(3)$ gauge theory description and the $\mathrm{Sp}(2)$ can be understood in terms of Hanany-Witten moves from a point of view of the $(p, q) 5$-branes web [24]. In the geometric M-theory description this correspond to some flop transitions together with some complex structure deformations, and it should work similarly to the duality between the SU and Sp descriptions of the marginal theory for the rank two E-string, which we will discuss in more detail in section 3.3.1. 


\begin{tabular}{|c|c|c|c|c|}
\hline CFD for SCFT & Flavor & Gauge Theory & BPS Spin 0 & BPS Spin 1 \\
\hline & $\mathrm{E}_{8}$ & $\mathrm{SU}(2)+7 \mathbf{F}$ & 248 & 3875 \\
\hline & $\mathrm{E}_{7}$ & $\mathrm{SU}(2)+6 \mathbf{F}$ & 56 & 133 \\
\hline & $\mathrm{E}_{6}$ & $\mathrm{SU}(2)+5 \mathbf{F}$ & 27 & $\overline{27}$ \\
\hline (1)- & $\mathrm{SO}(10)$ & $\mathrm{SU}(2)+4 \mathbf{F}$ & 16 & 10 \\
\hline & $\mathrm{SU}(5)$ & $\mathrm{SU}(2)+3 \mathbf{F}$ & 10 & $\overline{5}$ \\
\hline & $\mathrm{SU}(3) \times \mathrm{SU}(2)$ & $\mathrm{SU}(2)+2 \mathbf{F}$ & $(3,2)$ & $(\overline{\mathbf{3}}, \mathbf{1})$ \\
\hline-0 & $\mathrm{SU}(2) \times \mathrm{U}(1)$ & $\mathrm{SU}(2)+1 \mathbf{F}$ & $\mathbf{1}_{-1}, \mathbf{2}_{1}$ & $\mathbf{2}_{0}$ \\
\hline (1)-0 & $\mathrm{SU}(2)$ & $\mathrm{SU}(2)_{0}$ & & 2 \\
\hline 1) & $\mathrm{U}(1)$ & $\mathrm{SU}(2)_{\pi}$ & 1 & 0 \\
\hline (1) & - & - & & \\
\hline
\end{tabular}

Table 4. All 5d rank one SCFTs and the lowest spin BPS states from M2-brane wrapping modes over genus zero curves. We also list their flavor symmetry, gauge theory description.

6. Finally, there is the $6 \mathrm{~d}$ theory, with tensor branch

$$
\left[\mathfrak{s u}_{2}\right]-\stackrel{\mathfrak{s u}_{2}}{2}-\stackrel{\mathfrak{s u}_{2}}{2}-\left[\mathfrak{s u}_{2}\right]
$$

Upon circle compactification and $\mathbb{Z}_{2}$-automorphism twist the $5 \mathrm{~d}$ marginal theory data are given by

$$
\begin{array}{|c|}
\hline \text { Gauge Theory } \\
\hline \mathrm{SU}(3)_{0}+1 \mathbf{S y m}+1 \mathbf{F} \\
\hline
\end{array}
$$

There are two more theories, which are listed in [12], that could potentially give new $5 \mathrm{~d}$ rank two theories: $\mathrm{SU}(3)$ theory on a -3 curve, whose 5 d marginal theory is $\mathrm{SU}(3)_{9}$, however this does not give rise to any new $5 \mathrm{~d}$ descendant SCFTs.

The tensor branch theories, described by the resolved base geometries (A.5), (A.7), and (A.9), blow down to a smooth base, whereas (A.11), (A.13) and (A.15) all blow down to a singular base. In the former case, the non-minimal singularity can be directly resolved into a non-flat fibrations, without any base blow-ups, where in the latter the base needs to be resolved as well. 


\section{A.2 Summary tables for rank one and rank two 5d SCFTs}

In this appendix, we summarize our findings in rank one and two, by tabulating all $5 \mathrm{~d}$ SCFTs organized by $M$, the number of mass deformations. The tables contain the CFDs (in cases when there are different realizations we give all CFDs), their weakly-coupled gauge theory descriptions, the strongly coupled flavor symmetry as read off from the CFD, and the spin 0 and 1 BPS states. Model 3/4 refer to the marginal theories (A.10) and (A.11), respectively.

The CFDs in the following tables are connected by CFD-transitions, and these are shown in figures are shown in figure 10 for rank one, and in figures 11, 12, 13 for rank two.

\section{B Rank two prepotentials}

Using the matching of prepotentials (2.17), one can easily extract, for a given geometry, the relevant field theory data. In particular, we are interested in the number $N_{\mathbf{R}}$ of hypermultiplets in a representation $\mathbf{R}$ as well as the discrete Chern-Simons levels. For a given gauge group $G_{\text {gauge }}$, in most instances, these determine the effective gauge theory uniquely.

In this appendix we will consider the three types of rank two gauge theories that can be realized in terms of non-flat elliptic fibrations. These are $G_{\text {gauge }}=\mathrm{SU}(3)$ with $N_{f}$ fundamental hypers and Chern-Simons level $k, G_{\text {gauge }}=\operatorname{Sp}(2)$ with $N_{f}$ fundamental and $N_{a}$ anti-symmetric hypers, and $G_{\text {gauge }}=\mathrm{SU}(2)_{1} \times \mathrm{SU}(2)_{2}$ with $N_{f_{i}}$ fundamental hypers charged under $\mathrm{SU}(2)_{i}$. These gauge theories are effective low-energy descriptions of most of the rank two descendant 5d SCFTs, which we study in the main text.

There are two furhter gauge theory descriptions for rank two descendants: $G_{\text {gauge }}=G_{2}$ with $N_{7}$ hypermultiplets in the 7-dimensional representation of $G_{2}$, and $G_{\text {gauge }}=\mathrm{SU}(3)_{k}$ with $N_{f}$ fundamental hypers and $N_{s}$ hypermultiplets in the symmetric representation. These are either alternative dual effective models of the rank two gauge theories that we study here, or they describe the low-energy of the single descendant 5d SCFT outlying our analysis. We will come back to the detailed prepotential analysis of these theories in [31].

To begin with, note that we can simplify the field theory part on the right-hand side of $(2.17)$ to

$$
6 \mathcal{F}^{(4)}=k d_{i j l} \phi^{i} \phi^{j} \phi^{l}+\frac{1}{2}\left(\sum_{\alpha_{i} \in \Phi_{+}} 2(\alpha \cdot \phi)^{3}-\sum_{f} \sum_{\lambda_{f} \in \mathbf{W}_{f}} \sigma_{\lambda_{f}}\left(w \cdot \phi+m_{f}\right)^{3}\right),
$$

where $\Phi_{+}$are the positive roots, and

$$
\begin{aligned}
& \lambda_{f} \cdot \phi+m_{f}>0 \rightarrow \sigma_{\lambda_{f}}=1, \\
& \lambda_{f} \cdot \phi+m_{f}<0 \rightarrow \sigma_{\lambda_{f}}=-1,
\end{aligned}
$$

and $d_{i j l}=0$ for any $G$ but $G=\mathrm{SU}(3)$, such that $k$ is only relevant for $\mathrm{SU}(3)$ in this case. The resulting expression as a function in $\phi^{i}$ has to be identified with

$$
\begin{aligned}
\mathcal{F}_{\text {geo }} & =S_{1}^{3} \times\left(\phi^{1}\right)^{3}+3 S_{1}^{2} \cdot S_{2} \times\left(\phi^{1}\right)^{2} \phi^{2}+3 S_{1} \cdot S_{2}^{2} \times \phi^{1}\left(\phi^{2}\right)^{2}+S_{2}^{3} \times\left(\phi^{2}\right)^{3} \\
& \equiv\left(\phi^{1} S_{1}+\phi^{2} S_{2}\right)^{3} .
\end{aligned}
$$




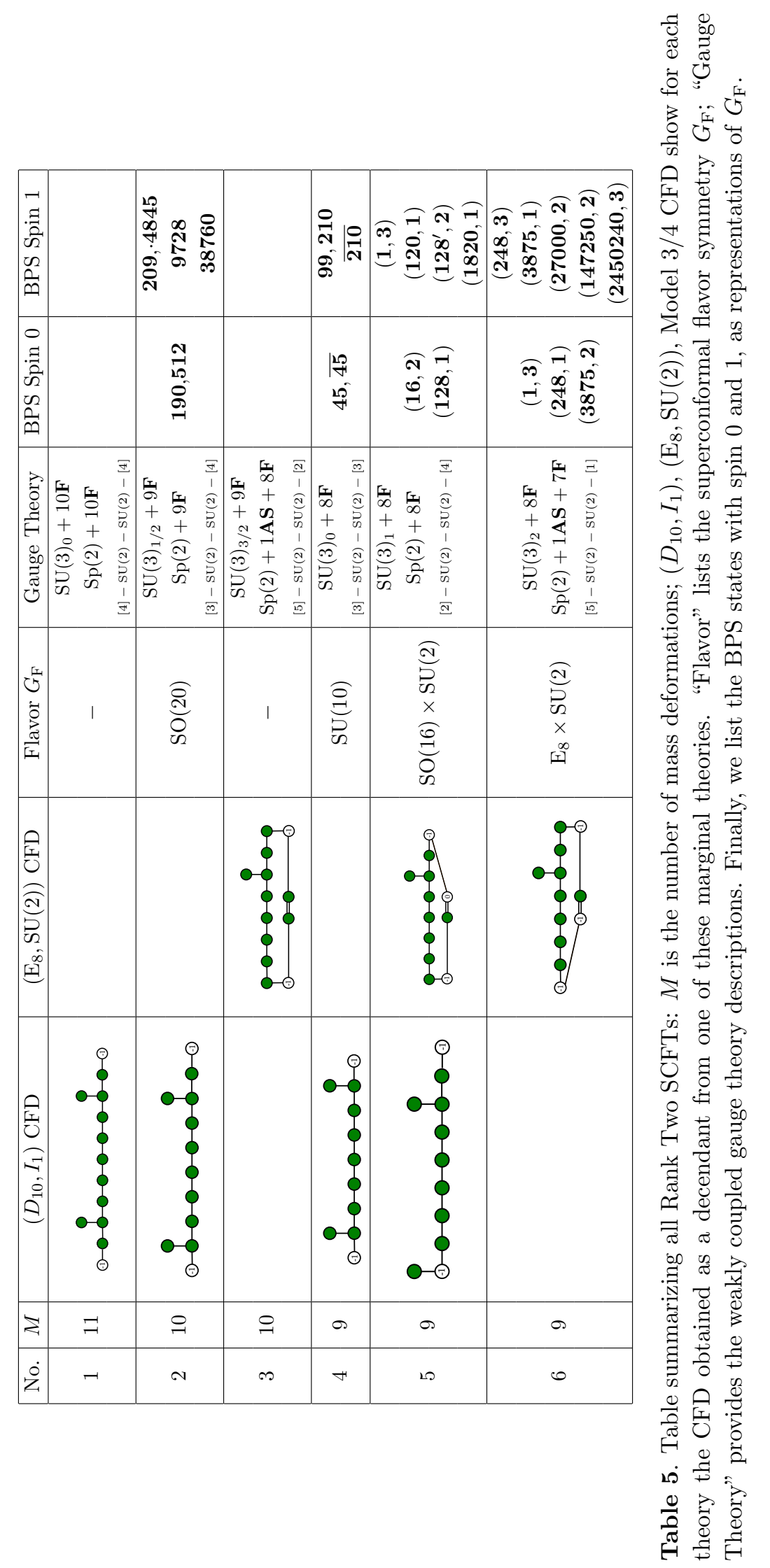




\begin{tabular}{|c|c|c|c|c|c|c|c|c|c|}
\hline 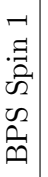 & 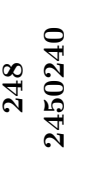 & 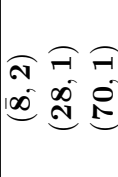 & 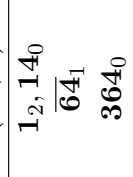 & 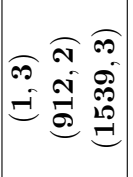 & 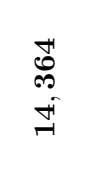 & 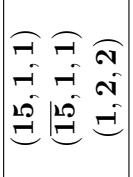 & $\begin{array}{ll}1 & 0 \\
0 & 0\end{array}$ & 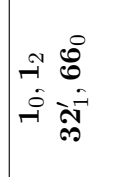 & 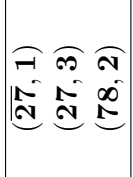 \\
\hline 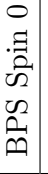 & $\begin{array}{l}\stackrel{20}{\infty} \\
\substack{\infty \\
\infty}\end{array}$ & 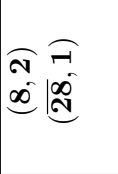 & $\vec{\nexists} \overrightarrow{-1}$ & 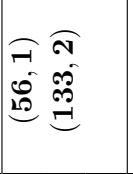 & ठే & 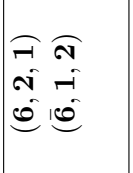 & 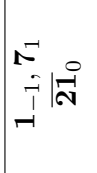 & 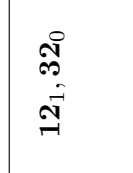 & 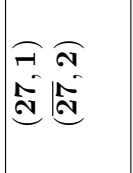 \\
\hline 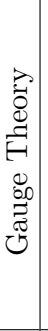 & 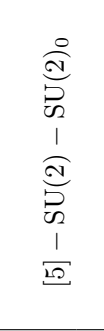 & 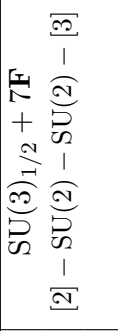 & 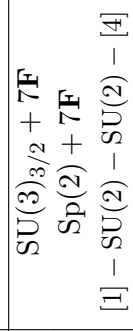 & 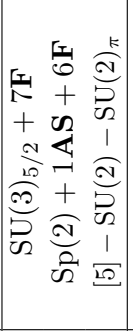 & 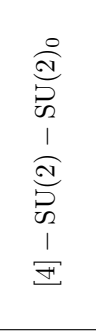 & 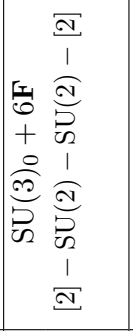 & 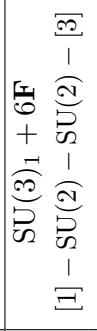 & 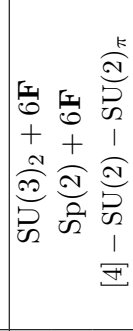 & 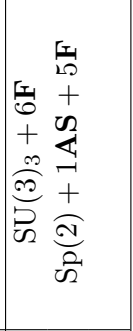 \\
\hline 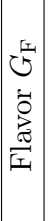 & [1 & 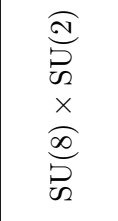 & 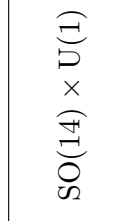 & 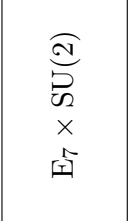 & 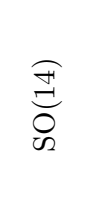 & 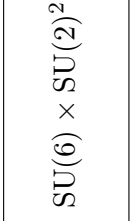 & 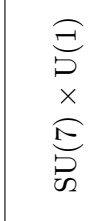 & 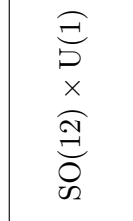 & 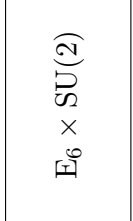 \\
\hline 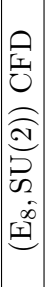 & $\begin{array}{c}0-0 \\
0 \\
0 \\
0 \\
0 \\
0 \\
0 \\
0 \\
0\end{array}$ & $\begin{array}{l}9 \\
0 \\
0 \\
0 \\
1 \\
0 \\
0 \\
0-0\end{array}$ & $\begin{array}{r}0 \\
0 \\
0 \\
0 \\
0 \\
9 \\
9 \\
0\end{array}$ & $\begin{array}{c}0-0 \\
0 \\
0-0 \\
00 \\
0 \\
0 \\
0\end{array}$ & $\begin{array}{r}0 \\
0 \\
0 \\
0 \\
0 \\
0 \\
0 \\
0\end{array}$ & $\begin{array}{r}0-9 \\
90 \\
10 \\
1 \\
0 \\
0-0\end{array}$ & & $\begin{array}{r}9 \\
0 \\
0 \\
9 \\
9 \\
90 \\
9 \\
0\end{array}$ & $\begin{array}{l}9-9 \\
0 \\
0 \\
0 \\
0 \\
9 \\
0\end{array}$ \\
\hline 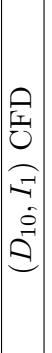 & & $\begin{array}{r}0 \\
0-9 \\
0 \\
0 \\
0 \\
0 \\
0 \\
0-0\end{array}$ & $\begin{array}{r}9 \\
0 \\
0-9 \\
0 \\
9 \\
9 \\
9 \\
0\end{array}$ & & $\begin{array}{r}9 \\
0 \\
0-0 \\
9 \\
0 \\
9 \\
0\end{array}$ & $\begin{array}{r}0-9 \\
0 \\
0 \\
0 \\
0 \\
0 \\
0-0\end{array}$ & & $\begin{array}{r}9 \\
9 \\
0-0 \\
9 \\
9 \\
9 \\
0\end{array}$ & \\
\hline z & $\infty$ & $\infty$ & $\infty$ & $\infty$ & $\curvearrowright$ & r & $\curvearrowright$ & r & $\curvearrowright$ \\
\hline$\dot{s}$ & $\wedge$ & $\infty$ & $\sigma$ & 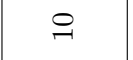 & $\exists$ & $\cong$ & 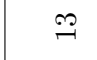 & 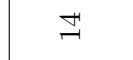 & $\stackrel{20}{-1}$ \\
\hline
\end{tabular}




\begin{tabular}{|c|c|c|c|c|c|c|c|c|c|c|}
\hline 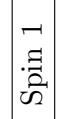 & & 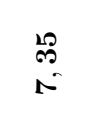 & 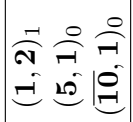 & ${ }_{0}^{-10}$ & 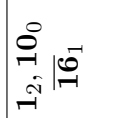 & 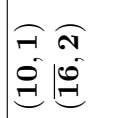 & 蛋 & 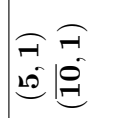 & 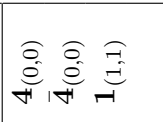 & $\begin{array}{l}\stackrel{\overrightarrow{\mathrm{N}}}{\vec{A}} \\
\vec{y}=0\end{array}$ \\
\hline 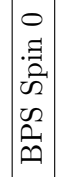 & & $\mid \vec{N}$ & 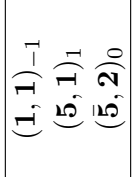 & 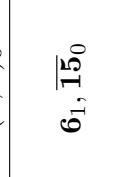 & $\begin{array}{l}\stackrel{0}{0} \\
\stackrel{+}{0} \\
\stackrel{0}{\oplus}\end{array}$ & 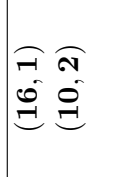 & ำ & $\underset{120}{N}$ & 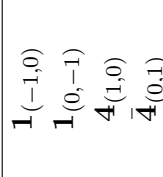 & 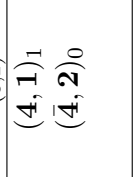 \\
\hline 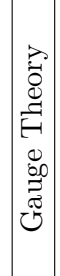 & 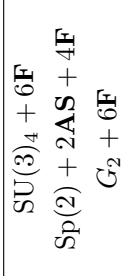 & 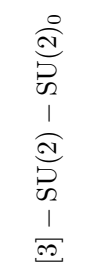 & 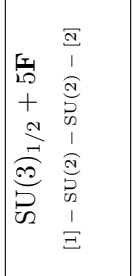 & 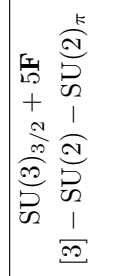 & 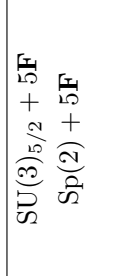 & 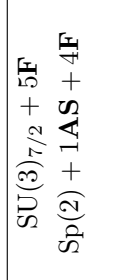 & 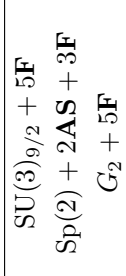 & 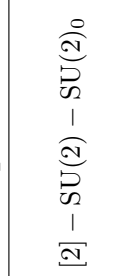 & 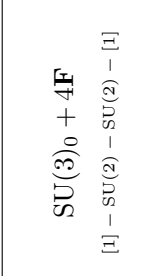 & 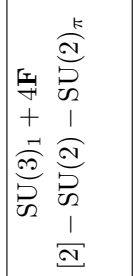 \\
\hline 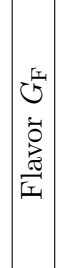 & 1 & E & 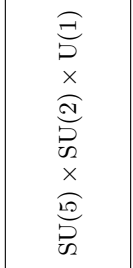 & 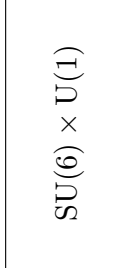 & 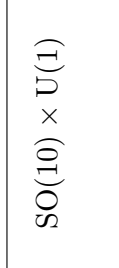 & 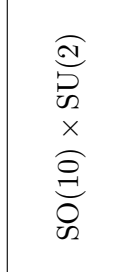 & $\begin{array}{l}\frac{6}{2} \\
\frac{2}{2}\end{array}$ & 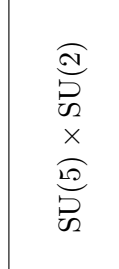 & 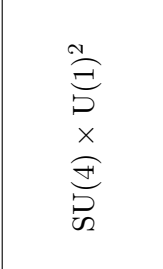 & 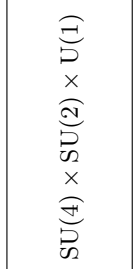 \\
\hline 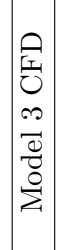 & $\begin{array}{r}0 \\
0 \\
0 \\
0 \\
0 \\
0\end{array}$ & & & & & $\begin{array}{r}0-0 \\
0 \\
0 \\
0 \\
0 \\
0-0\end{array}$ & $\begin{array}{r}0 \\
0 \\
0 \\
0 \\
0 \\
-0\end{array}$ & & & \\
\hline 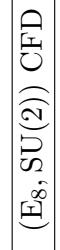 & & $\begin{array}{r}9 \\
0 \\
0 \\
1 \\
0 \\
0 \\
0\end{array}$ & $\begin{array}{r}0-9 \\
90 \\
10 \\
1 \\
0 \\
0\end{array}$ & $\begin{array}{r}9 \\
0 \\
0 \\
10 \\
0 \\
0\end{array}$ & $\begin{array}{r}0 \\
0 \\
0 \\
0 \\
0 \\
0 \\
0 \\
0\end{array}$ & $\begin{array}{r}9-0 \\
0 \\
0 \\
0 \\
0 \\
0-0\end{array}$ & & $\begin{array}{r}-9 \\
9 \\
1 \\
0 \\
0\end{array}$ & $\begin{array}{l}0 \\
9 \\
0 \\
0 \\
0 \\
0\end{array}$ & $\begin{array}{l}09 \\
90 \\
99 \\
9 \\
0\end{array}$ \\
\hline 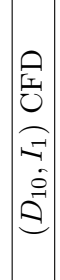 & & $\begin{array}{r}0 \\
0-9 \\
0 \\
0 \\
0 \\
0 \\
0\end{array}$ & $\begin{array}{r}0-9 \\
9 \\
9 \\
0 \\
0 \\
0\end{array}$ & $\begin{array}{r}0 \\
0 \\
0 \\
0 \\
0 \\
0 \\
0\end{array}$ & $\begin{array}{r}0 \\
0 \\
0 \\
0 \\
0 \\
9 \\
0\end{array}$ & & & $\begin{array}{r}0-9 \\
9 \\
0 \\
0 \\
0\end{array}$ & $\begin{array}{l}9 \\
0 \\
0 \\
0\end{array}$ & $\begin{array}{r}0-9 \\
0 \\
9 \\
9 \\
0\end{array}$ \\
\hline$z$ & $\wedge$ & 0 & 0 & 0 & 0 & 0 & 0 & 10 & 10 & 10 \\
\hline$\dot{0}$ & $\stackrel{\bullet}{-}$ & $\bumpeq$ & $\stackrel{\infty}{=}$ & $\stackrel{\rightarrow}{\rightarrow}$ & 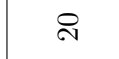 & $\overrightarrow{\mathrm{N}}$ & N & $\stackrel{2}{\sim}$ & $\overleftrightarrow{N}$ & $\stackrel{2}{N}$ \\
\hline
\end{tabular}




\begin{tabular}{|c|c|c|c|c|c|c|c|c|c|c|c|}
\hline $\mid \begin{array}{l}\mid \\
\text { : } \\
\hat{n}^{2}\end{array}$ & $\begin{array}{l}120 \\
10 \\
10\end{array}$ & 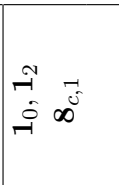 & 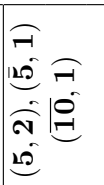 & 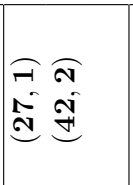 & $\begin{array}{c}\stackrel{0}{1} \\
\text { fo }\end{array}$ & 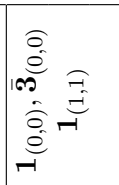 & 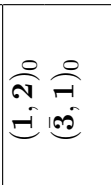 & $\begin{array}{c}\vec{A} \\
0 \\
-1\end{array}$ & $\begin{array}{l}\overrightarrow{\vec{H}} \\
\overrightarrow{-1}\end{array}$ & 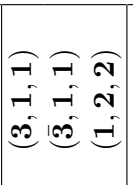 & $B=$ \\
\hline 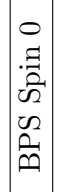 & $\begin{array}{l}\stackrel{0}{0} \\
=10\end{array}$ & $\begin{array}{c}0 \\
\infty^{\circ} \\
\overrightarrow{0} \\
\infty^{5}\end{array}$ & 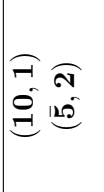 & 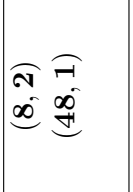 & 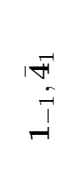 & 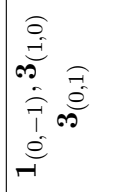 & 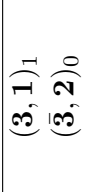 & $\begin{array}{l}8 \\
\dot{H}\end{array}$ & $\begin{array}{l}\text { मी } \\
\dot{\theta}\end{array}$ & 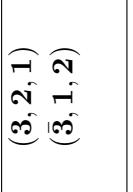 & $\stackrel{\overrightarrow{0}}{-}$ \\
\hline 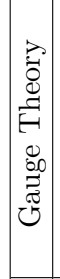 & 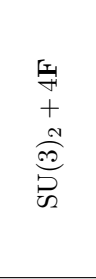 & 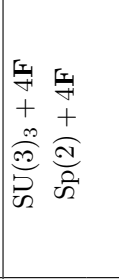 & 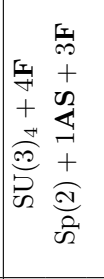 & 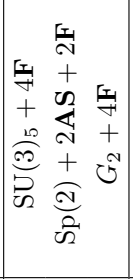 & 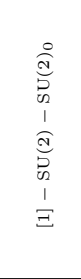 & 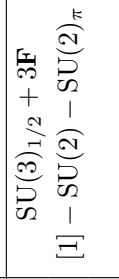 & 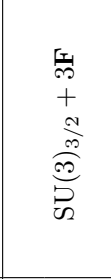 & 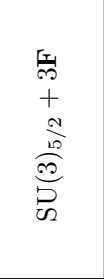 & 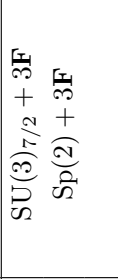 & 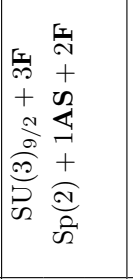 & 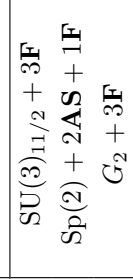 \\
\hline 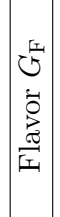 & $\begin{array}{l}\hat{E} \\
0 \\
x \\
x \\
0 \\
0 \\
0 \\
0\end{array}$ & 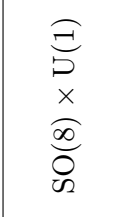 & 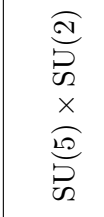 & 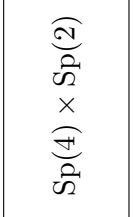 & 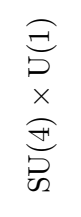 & 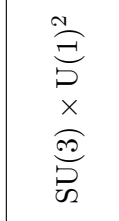 & 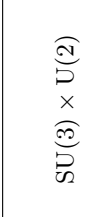 & 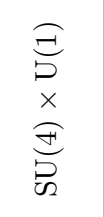 & $\begin{array}{l}\widehat{\Xi} \\
0 \\
x \\
x \\
0 \\
0 \\
0\end{array}$ & 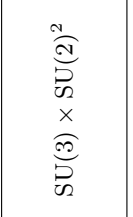 & 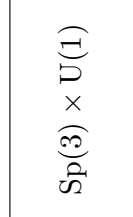 \\
\hline 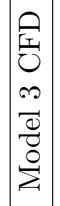 & & $\begin{array}{r}0 \\
0 \\
0 \\
0 \\
0 \\
0-0\end{array}$ & $\begin{array}{r}9-9 \\
0 \\
0 \\
9 \\
9\end{array}$ & $\begin{array}{r}-9 \\
9 \\
9 \\
0-\Theta\end{array}$ & & & & $\begin{array}{r}9 \\
0 \\
0 \\
0 \\
0-0 \\
0-0\end{array}$ & $\begin{array}{r}0 \\
0 \\
0-0 \\
9 \\
0 \\
0\end{array}$ & $\begin{array}{r}9 \\
9 \\
0-9\end{array}$ & $\begin{array}{r}9 \\
9 \\
9 \\
90\end{array}$ \\
\hline 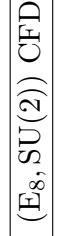 & $\begin{array}{l}9 \\
0 \\
9 \\
9 \\
0\end{array}$ & $\begin{array}{r}9 \\
0 \\
0-0 \\
9 \\
9-0 \\
0-0\end{array}$ & $\begin{array}{r}9-9 \\
0 \\
0 \\
9 \\
9\end{array}$ & & $\begin{array}{l}9 \\
\vdots \\
\vdots \\
0\end{array}$ & $\begin{array}{l}9 \\
9 \\
9 \\
9\end{array}$ & $\begin{array}{l}0.9 \\
90 \\
90 \\
0\end{array}$ & $\begin{array}{r}9 \\
0-0 \\
0 \\
0.0 \\
0-0\end{array}$ & $\begin{array}{r}9 \\
0 \\
0-0 \\
9 \\
9 \\
0\end{array}$ & of & \\
\hline 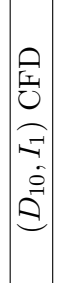 & $\begin{array}{r}9 \\
0 \\
\vdots \\
0 \\
0\end{array}$ & $\begin{array}{r}0 \\
0 \\
0 \\
0 \\
0 \\
0\end{array}$ & & & $\begin{array}{r}9 \\
0 \\
9 \\
0 \\
0\end{array}$ & $\begin{array}{l}9 \\
9 \\
0 \\
0\end{array}$ & $\begin{array}{r}0-0 \\
0 \\
0 \\
0\end{array}$ & $\begin{array}{r}9 \\
0 \\
0 \\
0\end{array}$ & $\begin{array}{r}9 \\
0 \\
0 \\
9 \\
0\end{array}$ & & \\
\hline$z$ & 10 & 10 & 10 & 10 & + & + & 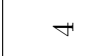 & H & 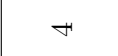 & 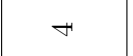 & 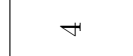 \\
\hline$\dot{\dot{z}}$ & $\stackrel{\sim}{\sim}$ & ล & $\stackrel{\infty}{\sim}$ & $\stackrel{\sim}{\mathrm{N}}$ & $\stackrel{\circ}{\infty}$ & $\vec{\infty}$ & ๙ิ & $\dddot{\circ}$ & $\ddot{s}$ & $\stackrel{8}{\circ}$ & $\ddot{\infty}$ \\
\hline
\end{tabular}




\begin{tabular}{|c|c|c|c|c|c|c|c|c|c|c|c|}
\hline $\begin{array}{l}-\vec{z} \\
. \bar{z} \\
\tilde{a}\end{array}$ & & $\stackrel{+}{\rightarrow}$ & $\begin{array}{l}0 \\
0 \\
0 \\
-1\end{array}$ & 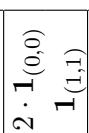 & $\underset{\stackrel{e}{e}}{\stackrel{a}{v}}$ & $\begin{array}{l}\stackrel{0}{\vec{A}} \\
\stackrel{\vec{N}}{=}\end{array}$ & 100 & 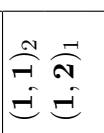 & 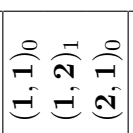 & fi & $\vec{r}$ \\
\hline 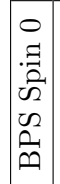 & & & 100 & 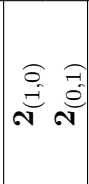 & 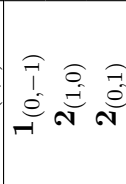 & 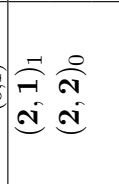 & $\begin{array}{l}-1 \\
\dot{0} \\
\infty\end{array}$ & 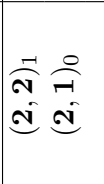 & 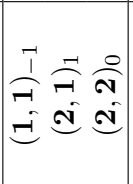 & $\vec{H} \overrightarrow{4}$ & 0 \\
\hline 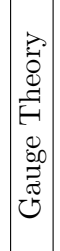 & 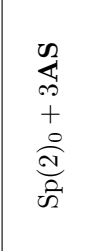 & 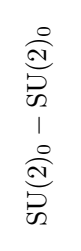 & 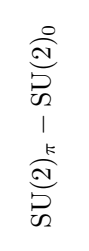 & 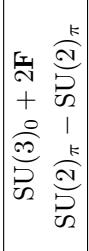 & 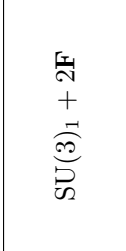 & 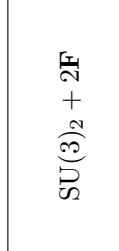 & 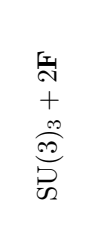 & 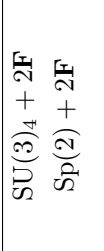 & 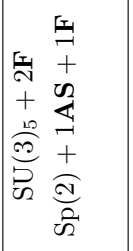 & 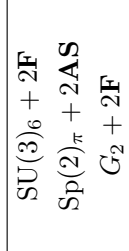 & 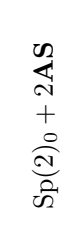 \\
\hline 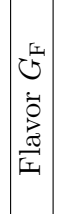 & 1 & 泀 & 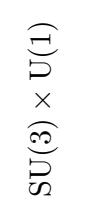 & 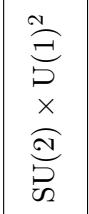 & 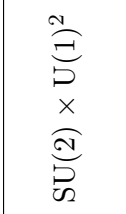 & 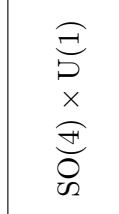 & 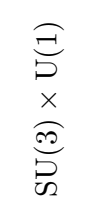 & 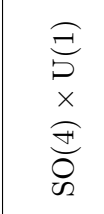 & 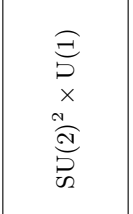 & 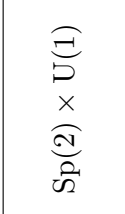 & $\begin{array}{l}\widehat{0} \\
\text { कू } \\
\text { की }\end{array}$ \\
\hline 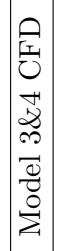 & $\begin{array}{r}\text { if } \\
0 \\
0 \\
0 \\
0 \\
0\end{array}$ & & & & & $\begin{array}{r}0-9 \\
0 \\
0-0\end{array}$ & $\begin{array}{r}9 \\
0-p \\
90 \\
9\end{array}$ & $\begin{array}{r}0 \\
0 \\
0 \\
-0 \\
9 \\
0\end{array}$ & $\begin{array}{l}0 \\
0 \\
0 \\
0\end{array}$ & - & $\begin{array}{r}9 \\
0-0 \\
0 \\
0=0\end{array}$ \\
\hline 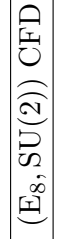 & & 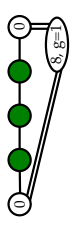 & $\begin{array}{l}0 \\
9 \\
0 \\
0\end{array}$ & $\begin{array}{l}0 \\
0 \\
0 \\
0\end{array}$ & $\begin{array}{l}90 \\
90 \\
0 \\
0\end{array}$ & $\begin{array}{r}0-9 \\
90 \\
0-0\end{array}$ & $\begin{array}{r}9 \\
0-9 \\
9 \\
0\end{array}$ & $\begin{array}{r}0 \\
0 \\
0-9 \\
0 \\
0 \\
0\end{array}$ & $\int_{0}^{+\infty}$ & & \\
\hline 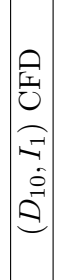 & & $\begin{array}{l}9 \\
0 \\
0 \\
0 \\
0\end{array}$ & $\begin{array}{l}0 \\
9 \\
0\end{array}$ & $\begin{array}{l}9 \\
9 \\
9 \\
9 \\
0\end{array}$ & 9 & $\begin{array}{r}0-9 \\
9 \\
9\end{array}$ & $\begin{array}{r}0 \\
0 \\
0 \\
0\end{array}$ & $\begin{array}{r}9 \\
0 \\
0-9\end{array}$ & & & \\
\hline$z$ & 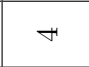 & $\infty$ & $\infty$ & $\infty$ & $\infty$ & $\infty$ & $\infty$ & $\infty$ & $\infty$ & $\infty$ & $\infty$ \\
\hline$\dot{\check{z}}$ & مै & $\infty$ & $\mathscr{~}$ & 아 & $F$ & F & $\Re$ & $\not$ & $\stackrel{2}{9}$ & f & 与 \\
\hline
\end{tabular}




\begin{tabular}{|c|c|c|c|c|c|c|c|c|c|c|c|c|}
\hline $\begin{array}{l}\vec{\nexists} \\
\text { है } \\
\text { है }\end{array}$ & ণi & 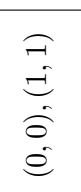 & 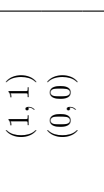 & $\vec{N}$ & $\vec{N}$ & 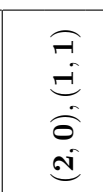 & $\begin{array}{l}\vec{N} \\
\hat{0}\end{array}$ & $\vec{N}$ & 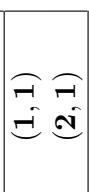 & 0 & - & $\overrightarrow{0}$ \\
\hline 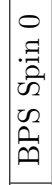 & $\vec{N}$ & $\begin{array}{l}\vec{E} \\
\dot{e} \\
\dot{a} \\
\dot{0}\end{array}$ & 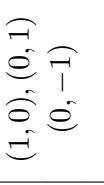 & 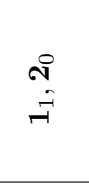 & $\begin{array}{l}\vec{N} \\
\hat{i}\end{array}$ & 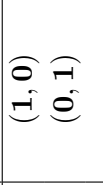 & 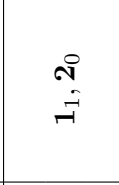 & 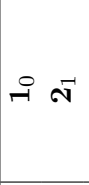 & $\begin{array}{l}\text { ลิ } \\
\text { â }\end{array}$ & - & & \\
\hline 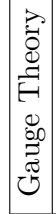 & 1 & 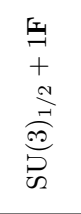 & 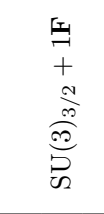 & 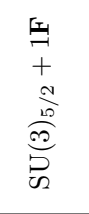 & 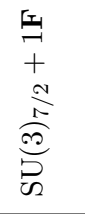 & 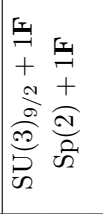 & 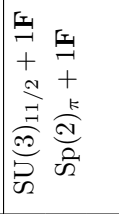 & 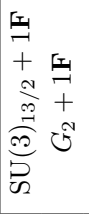 & 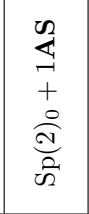 & 1 & 兽 & $\stackrel{\overrightarrow{0}}{\overrightarrow{0}}$ \\
\hline 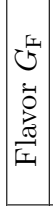 & 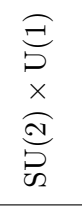 & 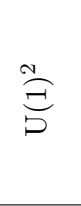 & $\stackrel{n}{\vec{E}}$ & 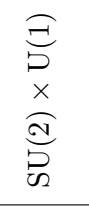 & 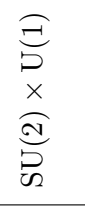 & 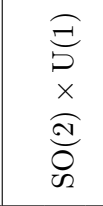 & 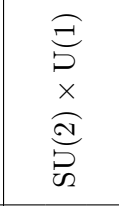 & 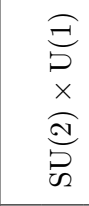 & 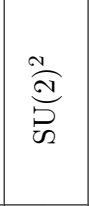 & $\widehat{\vec{\Xi}}$ & $\widehat{\Xi}$ & $\stackrel{\Xi}{\vec{\sigma}}$ \\
\hline 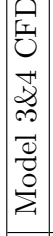 & & & $\begin{array}{l}90 \\
90\end{array}$ & 9? & - & ? & $\theta=\sqrt{\theta-\theta}$ & $\theta^{-9}$ & $\begin{array}{l}9 \\
0 \\
0 \\
0-0\end{array}$ & 8 & & ? \\
\hline 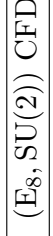 & $\begin{array}{l}0 \\
0 \\
0 \\
0\end{array}$ & $\begin{array}{l}8 \\
98 \\
9\end{array}$ & $\begin{array}{l}0 \\
90 \\
0-0\end{array}$ & 9. & -9 & - & $0=\int_{0}^{-\theta}$ & & $\int_{0}^{0-9}$ & 8 & $\begin{array}{l}8 \\
0 \\
0\end{array}$ & ] \\
\hline 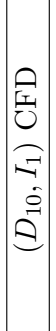 & $\begin{array}{l}9 \\
0 \\
0\end{array}$ & $\begin{array}{l}9 \\
9 \\
9\end{array}$ & 9 & 9 & 9 & $\odot$ & & & & @ & 9 & 1 \\
\hline$z$ & $\curvearrowright$ & $\curvearrowright$ & N & N & N & N & $\sim$ & N & N & - & - & $\neg$ \\
\hline$\dot{0}$ & $\stackrel{\infty}{\leftrightarrow}$ & $\stackrel{7}{7}$ & 80 & 20 & iิ & $\ddot{10}$ & 20 & 28 & $\stackrel{\circ}{\circ}$ & 合 & $\infty$ & 88 \\
\hline
\end{tabular}




\begin{tabular}{|c|c|c|c|c|c|c|c|c|c|}
\hline \begin{tabular}{|l|} 
\\
$\vec{a}$ \\
$\vec{n}$ \\
$\tilde{n}$
\end{tabular} & - & N & - & $\stackrel{\sim}{\sim}$ & $\boldsymbol{N}$ & $\ddot{\dot{N}}$ & - & & \\
\hline $\begin{array}{c}0 \\
: a \\
\tilde{a} \\
\tilde{n} \\
\tilde{\omega} \\
\tilde{\omega} \\
\infty\end{array}$ & & & $\neg$ & & & & 0 & & \\
\hline 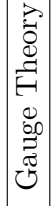 & 永 & 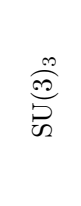 & 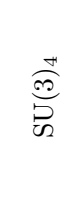 & 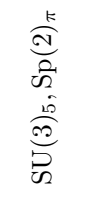 & 总 & 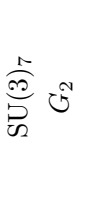 & $\begin{array}{l}\stackrel{0}{\mathrm{~d}} \\
\stackrel{2}{a}\end{array}$ & 1 & 1 \\
\hline 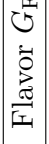 & $\underset{\partial}{\vec{D}}$ & $\begin{array}{l}\widehat{N} \\
\stackrel{D}{D}\end{array}$ & $\widehat{\Xi}$ & $\widehat{E}$ & 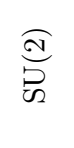 & $\widehat{E}$ & $\widehat{\Xi}$ & 1 & 1 \\
\hline 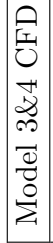 & 9? & & - & & & 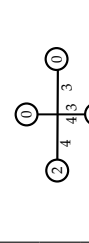 & $\begin{array}{l}9 \\
9 \\
0=0\end{array}$ & O= II & $\prod_{\theta=0}^{9}$ \\
\hline 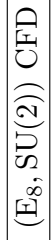 & शु? & & 8 & & $=$ & & ? & Y= (I) & $\mathbb{8}$ \\
\hline 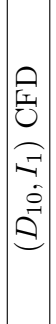 & $\prod_{0}^{0-\theta}$ & 0 & ${ }_{0}^{9}$ & ${ }_{0}^{0}$ & & & $\prod_{0}^{9}$ & $\prod_{0}^{9}$ & $i$ \\
\hline$z$ & $\neg$ & $\neg$ & $\neg$ & - & - & - & - & 0 & 0 \\
\hline$\stackrel{\circ}{z}$ & 8 & $\overrightarrow{0}$ & ชิ & $\because$ & $\vec{\sigma}$ & 18 & $\because$ & $\overleftarrow{6}$ & $\stackrel{\infty}{0}$ \\
\hline
\end{tabular}


SU(3). The cubic part of the prepotential of SU(3) gauge theory with general ChernSimons level $k$ and $N_{f}$ hypermultiplets in the fundamental is

$$
\begin{aligned}
6 \mathcal{F}_{\mathrm{SU}(3)}^{(3)}= & 3 k\left(\left(\phi^{1}\right)^{2}\left(\phi^{2}\right)-\phi^{2}\left(\phi^{1}\right)^{2}\right) \\
& +\left|\left[\begin{array}{c}
2 \\
-1
\end{array}\right]^{T}\left[\begin{array}{l}
\phi^{1} \\
\phi^{2}
\end{array}\right]\right|^{3}+\left|\left[\begin{array}{l}
1 \\
1
\end{array}\right]^{T}\left[\begin{array}{c}
\phi^{1} \\
\phi^{2}
\end{array}\right]\right|^{3}+\left|\left[\begin{array}{c}
-1 \\
2
\end{array}\right]^{T}\left[\begin{array}{c}
\phi^{1} \\
\phi^{2}
\end{array}\right]\right|^{3} \\
& -\frac{1}{2}\left(\sum_{i}^{N_{f}} \sigma_{1}^{i}\left(\phi^{1}+m_{f}^{i}\right)^{3}+\sigma_{2}^{i}\left(\phi^{2}-\phi^{1}+m_{f}^{i}\right)^{3}+\sigma_{3}^{i}\left(-\phi^{2}+m_{f}^{i}\right)^{3}\right),
\end{aligned}
$$

where the $\sigma_{w}^{i}$ can be \pm 1 and $w=1,2,3$ labels the positive weights of the fundamental of $\mathrm{SU}(3)$. In particular we would like to compare this with the (B.3) to obtain the CS-level and fix the sign of the terms in the third line.

We can now assume that $\sigma_{1}^{i}$ and $\sigma_{3}^{i}$ have opposite signs for all $i$, in particular, $\sigma_{1}^{i}=$ $-\sigma_{3}^{i}=1$. For fixed $N_{f}$, this is a justified assumption since different sign combinations will lead to regions of validity for $\left\{\phi^{1}, \phi^{2}\right\}$ outside the Weyl chamber

$$
2 \phi^{1}-\phi^{2} \geq 0, \quad \phi^{1}+\phi^{2} \geq 0, \quad-\phi^{1}-2 \phi^{2} \geq 0 .
$$

In particular, other sign combinations of $\sigma_{1}^{i}$ and $\sigma_{3}^{i}$ will lead to a gauge theory with different $N_{f}$. We will give more details on this using the Box-Graph approach in [31].

The only ambiguity can now come from $\sigma_{2}^{i}$. We parametrize the number of flavors with $\sigma_{2}^{i}=-1$ by $a$; having carefully kept track of the signs, we can for now ignore the terms proportional to $m_{f}^{i}$ and $\left(m_{f}^{i}\right)^{2}$, then (B.4) becomes

$$
\begin{aligned}
6 \mathcal{F}_{\mathrm{SU}(3)}^{(3)}= & 3 k\left(\left(\phi^{1}\right)^{2} \phi^{2}-\left(\phi^{2}\right)^{2} \phi^{1}\right)+\left(8\left(\phi^{1}\right)^{3}-3\left(\phi^{1}\right)^{2} \phi^{2}-3\left(\phi^{2}\right)^{2} \phi^{1}+8\left(\phi^{2}\right)^{3}\right) \\
& -\frac{N_{f}-a}{2}\left(\left(\phi^{1}\right)^{3}+\left(\phi^{2}-\phi^{1}\right)^{3}-\left(-\phi^{2}\right)^{3}\right)-\frac{a}{2}\left((\phi)^{3}-\left(\phi^{2}-\phi^{1}\right)^{3}-\left(-\phi^{2}\right)^{3}\right) .
\end{aligned}
$$

By comparing the cubic terms in $\left(\phi^{1}\right)$ and $\left(\phi^{2}\right)$ of this expression with (B.3) we get the following equations

$$
\left(\begin{array}{cc}
8-a & -a+\frac{N_{f}}{2}-1+k \\
a-\frac{N_{f}}{2}-1-k & a-N_{f}+8
\end{array}\right)=\left(\begin{array}{cc}
S_{1}^{3} & S_{1} S_{2}^{2} \\
S_{2} S_{1}^{2} & S_{2}^{3}
\end{array}\right) .
$$

which fix the values of $N_{f}, k, a$. Furthermore, (B.7) and (2.14) imply that $g\left(\Sigma_{12}\right)=0$, and finally the total number of mass deformations is

$$
M=N_{f}+1
$$

where the +1 accounts for the instanton $\mathrm{U}(1)_{T}$ associated to $G_{\text {gauge }}=\mathrm{SU}(3)$. 
$\mathbf{S U}(2)_{1} \times \mathbf{S U}(2)_{2}$. In this case there is no Chern-Simons level to determine. The cubic field theory prepotential for generic $N_{f_{1}}, N_{f_{2}}$ reads

$$
6 \mathcal{F}_{\mathrm{SU}(2)^{2}}^{(3)}=\left|2 \phi^{1}\right|^{3}+\left|2 \phi^{2}\right|^{3}-\left|\phi^{1}+\phi^{2}\right|^{3}-\left|\phi^{1}-\phi^{2}\right|^{3}-\sum_{i=1}^{N_{f_{1}}}\left|\phi^{1}+m_{f_{2}}^{i}\right|^{3}-\sum_{i=1}^{N_{f_{2}}}\left|\phi^{2}+m_{f_{2}}^{i}\right|^{3} .
$$

We consider the chamber $\phi^{1} \geq 0, \phi^{2} \geq 0$, and we explicitly write the sign dependence like in (B.1), such that

$$
6 \mathcal{F}_{\mathrm{SU}(2)-\mathrm{SU}(2)}^{(3)}=8\left(\phi^{1}\right)^{3}+8\left(\phi^{2}\right)^{3}-\left(\phi^{1}+\phi^{2}\right)^{3}-\sigma_{0}\left(\phi^{1}-\phi^{2}\right)^{3}-\sum_{i=1}^{N_{f_{1}}} \sigma_{1}^{i}\left(\phi^{1}\right)^{3}-\sum_{i=1}^{N_{f_{2}}} \sigma_{2}^{i}\left(\phi^{2}\right)^{3},
$$

where we also ignored the terms proportional to $m_{f_{1}}^{i}, m_{f_{2}}^{i}$ and $\left(m_{f_{1}}^{i}\right)^{2},\left(m_{f_{2}}^{i}\right)^{2}$. Similarly to the $\mathrm{SU}(3)$ case for determined values of $N_{f_{1}}$ and $N_{f_{2}}$ the conditions $\phi^{1} \geq 0, \phi^{2} \geq 0$ fix $\sigma_{1}^{i}=\sigma_{2}^{i}=1$. Having set this we can now compare (B.10) with the geometric quantity (B.3). We get the following equations

$$
\left(\begin{array}{cc}
7-l_{0}-N_{f} & -1-l_{0} \\
l_{0}-1 & 7+l_{0}-N_{f_{1}}
\end{array}\right)=\left(\begin{array}{cc}
S_{1}^{3} & S_{1} S_{2}^{2} \\
S_{2} S_{1}^{2} & S_{2}^{3}
\end{array}\right)
$$

These equations completely fix $N_{f_{1}}, N_{f_{2}}, \sigma_{0}$. Moreover, (B.11) and (2.14) imply again that $g\left(\Sigma_{12}\right)=0$. The total number of mass deformation is given by

$$
M=N_{f_{1}}+N_{f_{1}}+3
$$

where the +3 accounts for the two instanton $\mathrm{U}(1)_{T_{1,2}}$ associated to $\mathrm{SU}(2)_{1,2}$, and for the $\mathrm{SU}(2)$ baryonic symmetry rotating the hypermultiplet in the bifundamental of $\mathrm{SU}(2)_{1} \times \mathrm{SU}(2)_{2}$.

$\mathbf{S p}(2)$. The prepotential for $\operatorname{Sp}(2)(\equiv \mathrm{SO}(5))$ gauge theory with $N_{f}$ hypermultiplets in the fundamental and $N_{a}$ in the antisymmetric is

$$
\begin{aligned}
6 \mathcal{F}_{\mathrm{Sp}(2)}^{(3)}= & \left|\left[\begin{array}{l}
2 \\
0
\end{array}\right]^{T}\left[\begin{array}{l}
\phi^{1} \\
\phi^{2}
\end{array}\right]\right|^{3}+\left|\left[\begin{array}{l}
0 \\
1
\end{array}\right]^{T}\left[\begin{array}{l}
\phi^{1} \\
\phi^{2}
\end{array}\right]\right|^{3}+\left|\left[\begin{array}{c}
-2 \\
2
\end{array}\right]^{T}\left[\begin{array}{c}
\phi^{1} \\
\phi^{2}
\end{array}\right]\right|^{3}+\left|\left[\begin{array}{c}
2 \\
-1
\end{array}\right]^{T}\left[\begin{array}{l}
\phi^{1} \\
\phi^{2}
\end{array}\right]\right|^{3} \\
& -\sum_{i=1}^{N_{a}}\left(||\left[\begin{array}{l}
0 \\
1
\end{array}\right]^{T}\left[\begin{array}{l}
\phi^{1} \\
\phi^{2}
\end{array}\right]+\left.m_{a}^{i}\right|^{3}+\left|\left[\begin{array}{c}
2 \\
-1
\end{array}\right]^{T}\left[\begin{array}{l}
\phi^{1} \\
\phi^{2}
\end{array}\right]+m_{a}^{i}\right|^{3}\right) \\
& -\sum_{i=1}^{N_{f}}\left(||\left[\begin{array}{l}
1 \\
0
\end{array}\right]^{T}\left[\begin{array}{l}
\phi^{1} \\
\phi^{2}
\end{array}\right]+\left.m_{f}^{i}\right|^{3}+\left|\left[\begin{array}{c}
-1 \\
1
\end{array}\right]^{T}\left[\begin{array}{l}
\phi^{1} \\
\phi^{2}
\end{array}\right]+m_{f}^{i}\right|^{3}\right)
\end{aligned}
$$

Also in this case there is no CS-level to determine. The cone defined by

$$
2 \phi^{1} \geq 0, \quad \phi^{2} \geq 0, \quad-2 \phi^{1}+2 \phi^{2} \geq 0, \quad 2 \phi^{1}-\phi^{2} \geq 0,
$$


specifies the signs of some of the absolute values in the second and third line of (B.13), such that the prepotential reads

$$
\begin{aligned}
6 \mathcal{F}_{\mathrm{Sp}(2)}^{(3)}= & \left(2 \phi^{1}\right)^{3}+\left(\phi^{2}\right)^{3}+\left(-2 \phi^{1}+2 \phi^{2}\right)^{3}+\left(2 \phi^{1}-\phi^{2}\right)^{3} \\
& -\left(N_{a}-a_{a}\right)\left(\left(\phi^{2}\right)^{3}+\left(2 \phi^{1}-\phi^{2}\right)^{3}\right)-a_{a}\left(\left(\phi^{2}\right)^{3}-\left(2 \phi^{1}-\phi^{2}\right)^{3}\right) \\
& -\left(N_{f}-a_{f}\right)\left(\left(\phi^{1}\right)^{3}+\left(-\phi^{1}+\phi^{2}\right)^{3}\right)-a_{f}\left(\left(\phi^{2}\right)^{3}-\left(-\phi^{1}+\phi^{2}\right)^{3}\right) .
\end{aligned}
$$

For our purpose, we can ignore the terms proportional to $m_{f}^{i}, m_{a}^{i}$ and $\left(m_{f}^{i}\right)^{2},\left(m_{a}^{i}\right)^{2}$, since we have kept track of the signs of the absolute values. By comparing the cubic expansion of this expression with the geometric quantity (B.3), we get the following constraints on $N_{f}, N_{a}, a_{f}, a_{a}$

$$
\left(\begin{array}{cc}
-2 a_{f}+16 a_{a}+8-8 N_{a} & -2 a_{f}+4 a_{a}-2 N_{a}+N_{f}-6 \\
2 a_{f}-8 a_{a}+4 N_{a}-N_{f}+4 & 2 a_{f}-2 a_{a}+8-N_{f}
\end{array}\right)=\left(\begin{array}{cc}
S_{1}^{3} & S_{1} S_{2}^{2} \\
S_{2} S_{1}^{2} & S_{2}^{3}
\end{array}\right) .
$$

This fixes $N_{a}, N_{f}, a_{f}, a_{a}$, where $a_{f}$ and $a_{a}$ can be half-integer, since $\operatorname{Sp}(2)$ has pseudo-real representations for the hypermultiplets. Although they can in principle take values from 0 to $N_{a}$ and 0 to $N_{f}$, respectively, only some of them are actually allowed and match the geometric prepotential. Using the Box-Graph approach, the set of positivity conditions of the Coulomb branch makes the bounds on the values of $\left\{a_{f}, a_{a}\right\}$ manifest. We will come back to this in [31]. At last, from (B.11) and (2.14) we can compute the genus of the gluing curve between the two surfaces, which reads

$$
g\left(\Sigma_{12}\right)=N_{a}-2 a_{a},
$$

and the total number of mass deformation is given by

$$
M=N_{f}+N_{a}+1,
$$

where the +1 accounts for the two instanton $\mathrm{U}(1)_{T}$ associated to $\operatorname{Sp}(2)$.

\section{Rational surfaces: dP versus gdP}

Generalized del Pezzo (gdP) surfaces play an essential part in the geometric description that we choose for 5d SCFTs in this paper. Here we provide some mathematical background on the rational surfaces. More details can be found in [97-99]. In the following we always use a smooth rational surface $S$ with an effective anticanonical divisor $-K_{S}$.

For a surface $S$ with $h^{1,1}(S)=r>2$, the Picard group of divisors on $S$ is an additive abelian group $\operatorname{Pic}(S)=\mathbb{Z}^{r}$ with the following generators

$$
h, e_{i}, \quad i=1, \ldots, r-1 .
$$

The intersection numbers between these are

$$
h^{2}=1, \quad h \cdot e_{i}=0, \quad e_{i} \cdot e_{j}=-\delta_{i j} .
$$

A general element of $\mathbb{Z}^{r}$ can be written as

$$
C=a h+\sum_{i=1}^{r-1} b_{i} e_{i}
$$



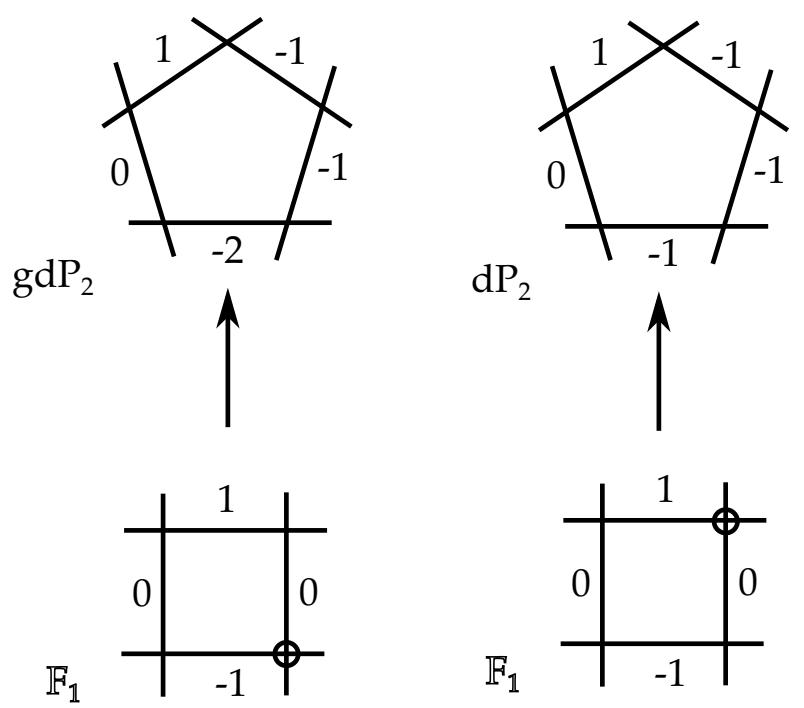

Figure 20. The blow-ups from the Hirzebruch surface $\mathbb{F}_{1}$ to a generalized del Pezzo surface gdP $\mathrm{P}_{2}$ of type $A_{1}$ and a del Pezzo surface $\mathrm{dP}_{2}$. The circle denotes the points to be blown up on $\mathbb{F}_{1}$. Hence $\operatorname{gdP} P_{2}$ and $\mathrm{dP}_{2}$ are related by a flop.

The anticanonical divisor of $S$ is

$$
-K_{S}=3 h-\sum_{i=1}^{r-1} e_{i}, .
$$

If $h^{1,1}(S)=r=2$, then $S$ is a Hirzebruch surface $\mathbb{F}_{n}$. If $n$ is even, then we need to use the following set of generators for $\operatorname{Pic}(S)$ rather than (C.2):

$$
s^{2}=-n, \quad s \cdot f=1, \quad f^{2}=0
$$

For odd $n$, we can use either (C.2) or (C.5). The anticanonical divisor of $\mathbb{F}_{n}$ is

$$
-K_{S}=2 s+(n+2) f
$$

If $h^{1,1}(S)=r=1$, then $S=\mathbb{P}^{2}$ and its Picard group is generated by the hyperplane class $h \subset \mathbb{P}^{2}$ with $h^{2}=1$.

The most essential characteristic of a rational surface are the generators of its Mori cone (or the cone of effective divisors as they are equivalent on a complex surface). For any rational surface $S$ with an effective anticanonical divisor $-K_{S}$ and $r>2$, its Mori cone is generated by a set, $\operatorname{Neg}(S)$, of irreducible rational curves $C$ with negative self-intersection. Recall that the genus $g$ of a curve $C$ on $S$ can be computed by the adjunction formula

$$
K \cdot C+C \cdot C=2 g-2
$$

A rational curve is defined to be a curve with genus $g=0$.

If $r=2$, then the Mori cone of $S=\mathbb{F}_{n}$ is generated by the $(-n)$-curve $s$ and the 0-curve $f$ in (C.5). If $r=1$, then the Mori cone of $S=\mathbb{P}^{2}$ is generated by $h$. 
We can further define the subset of $\operatorname{Neg}(S)$ : $\operatorname{Sing}(S)$ which is the set of irreducible rational curves with self-intersection $(-2)$ or lower. After $\operatorname{Sing}(S)$ is fixed, the set of $(-1)$ curves on $S$ is given by all the curves in the form of

$$
C=a h+\sum_{i=1}^{r-1} b_{i} e_{i}
$$

which satisfies

$$
K \cdot C=C \cdot C=-1
$$

and intersect all the curves in $\operatorname{Sing}(S)$ non-negatively [99]. This theorem is useful for generating the set of $(-1)$-curves on a rational surface.

Especially, when $\operatorname{Sing}(S)$ only contains $(-2)$-curves, $S$ is called a generalized del Pezzo surfaces (or weak-Fano surface). The generalized del Pezzo surfaces are comprehensively classified and studied in [97, 98]. We denote it by $\operatorname{gdP}_{n}$ if $n=h^{1,1}(S)-1$, which corresponds to the degree- $(9-n)$ cases in the mathematics literature. They are classified by the configuration of $(-2)$-curves, which form Dynkin diagrams (affine Dynkin diagrams for the cases of $\left.\mathrm{gdP}_{9}\right)$. For example, we call a $\mathrm{gdP}_{n}$ of type $A_{1}$ if there is only a single $(-2)$-curve on it that form an $A_{1}$ Dynkin diagram.

If $\operatorname{Sing}(S)$ is empty, such that there is no $(-2)$ or lower curve on $S$, then the surface $S$ is a del Pezzo surface $\mathrm{dP}_{n}$. The del Pezzo surface and generalized del Pezzo surfaces are related by a number of flops, see figure 20 for the case of $n=2$. One can also think generalized del Pezzo surfaces as special points in the complex structure moduli space of del Pezzo surfaces.

\section{Details of non-flat resolutions}

Various technical details for blow-ups of non-flat resolution of elliptic fibrations are collected in this appendix. We begin by deriving the two resolved geometries for the rank two Estring and the $\left(D_{10}, I_{1}\right)$ model, which will be the starting points for the flop chains for rank two theories, i.e. the marginal geometries, from which we read off the marginal (top) CFDs. These are precisely the fibers, where the non-flat surface components $S_{i}$ contain all the rational curves in the codimension one singular fibers. We also provide the marginal theories for higher rank conformal matter theories of type $\left(\mathrm{E}_{8}, \mathrm{SU}(n)\right)$ and $\left(\mathrm{E}_{n}, \mathrm{E}_{n}\right)$.

\section{D.1 Non-flat fiber resolutions}

\section{D.1.1 Rank one theories}

In this section, all non-flat fiber resolutions of the singular Tate model (3.8) for the rank one E-string are detailed. In the main text we already discussed the marginal geometry, where the non-flat fiber is $\mathrm{gdP}_{9}$, see (3.14). The descendant theories, which are the rank one $5 \mathrm{~d}$ SCFTs can either be obtained by flops of the $(-1)$ curve in $\mathrm{gdP}_{9}$, or using the direct resolutions, which we explain in the following: 
gdP $_{8}$ : to get a non-flat fiber with the topology of $\mathrm{gdP}_{8}$, we start with the Tate form (3.8) and do the following sequence of blow-ups

$$
\begin{gathered}
\left\{\{x, y, u, v, \delta\},\left\{x, y, U, u_{1}\right\},\left\{x, y, u_{1}, u_{2}\right\},\left\{y, u_{2}, u_{3}\right\},\left\{y, u_{1}, u_{3}, u_{4}\right\}\right. \\
\left\{y, u_{1}, u_{5}\right\},\left\{u_{1}, u_{3}, u_{6}\right\},\left\{u_{1}, u_{4}, u_{7}\right\},\left\{u_{1}, u_{5}, u_{8}\right\},\left\{u_{2}, u_{3}, u_{9}\right\},\left\{u_{3}, u_{4}, u_{10}\right\} \\
\left.\left\{u_{4}, u_{6}, u_{11}\right\},\left\{u_{3}, u_{6}, u_{12}\right\},\left\{u_{6}, u_{10}, u_{13}\right\},\left\{u_{10}, u_{12}, u_{14}\right\},\left\{u_{3}, u_{12}, u_{15}\right\}\right\}
\end{gathered}
$$

The first blow-up is a weighted blow-up,

$$
x \rightarrow x \delta^{3}, y \rightarrow y \delta^{2}, u \rightarrow U \delta, v \rightarrow V \delta .
$$

The exceptional divisor $\delta=0$ is a weighted projective space $\mathbb{P}^{1,1,2,3}$ in the ambient space, but its singular points do not intersect the hypersurface equation. After the replacement of variables, we divide the hypersurface equation by $\delta^{6}$, which preserves the Calabi-Yau condition of $Y$.

Denote by $S$ the non-flat fiber surface $\delta=0$ inside the resolved Calabi-Yau threefold. The intersection numbers of $S$ with the Cartan divisors associated to the affine $\mathrm{E}_{8}, D_{i}$, that are defined in (3.10), are

$$
S \cdot D_{i} \cdot D_{i}=(-1,-2,-2,-2,-2,-2,-2,-2,-2),(i=0, \ldots, 8) .
$$

Hence the flavor symmetry of the corresponding $5 \mathrm{~d}$ SCFT is $\mathrm{E}_{8}$ from the Dynkin diagram formed by the $(-2)$-curves.

$\mathbf{g d P}_{\mathbf{7}}-\mathbf{g d P}_{\mathbf{3}}$ : to obtain the subsequent geometries corresponds to performing a number of flops, which are essentially a choice of different orderings in the resolution sequences. Consider the sequence

$$
\begin{gathered}
\left\{\left\{x, y, u, u_{1}\right\},\left\{x, y, u_{1}, u_{2}\right\},\left\{y, u_{2}, u_{3}\right\}, \underline{\left\{u_{3}, v, \delta\right\}},\left\{y, u_{1}, u_{3}, u_{4}\right\}, \mathbf{\Phi}_{\mathbf{1}},\left\{y, u_{1}, u_{5}\right\},\right. \\
\left\{u_{1}, u_{3}, u_{6}\right\}, \mathbf{\Phi}_{\mathbf{2}},\left\{u_{1}, u_{4}, u_{7}\right\},\left\{u_{1}, u_{5}, u_{8}\right\},\left\{u_{2}, u_{3}, u_{9}\right\},\left\{u_{3}, u_{4}, u_{10}\right\}, \mathbf{\Phi}_{\mathbf{3}}, \\
\left.\left\{u_{4}, u_{6}, u_{11}\right\},\left\{u_{3}, u_{6}, u_{12}\right\}, \mathbf{\Phi}_{\mathbf{4}},\left\{u_{6}, u_{10}, u_{13}\right\},\left\{u_{10}, u_{12}, u_{14}\right\},\left\{u_{3}, u_{12}, u_{15}\right\}\right\},
\end{gathered}
$$

the non-flat fiber surface component $S: \delta=0$ is a $\mathrm{gdP}_{7}$. The intersection numbers of $S$ with the Cartan divisors are

$$
S \cdot D_{i} \cdot D_{i}=(0,-1,-2,-2,-2,-2,-2,-2,-2) \quad \Rightarrow G_{\mathrm{F}}=\mathrm{E}_{7} .
$$

For the other cases of $S=\mathrm{gdP}_{6}-\mathrm{gdP}_{3}$, we move the underlined blow-up $\left\{u_{3}, v, \delta\right\}$ to positions $\Phi_{1}, \Phi_{2}, \Phi_{3}, \Phi_{4}$. The intersection numbers of the non-flat fiber $S: \delta=0$ with the Cartan divisors in these cases are

$$
\begin{array}{lll}
\Phi_{1}: & S \cdot D_{i} \cdot D_{i}=(0,0,-1,-2,-2,-2,-2,-2,-2) & \Rightarrow G_{\mathrm{F}}=\mathrm{E}_{6} \\
\Phi_{2}: & S \cdot D_{i} \cdot D_{i}=(0,0,0,-1,-2,-2,-2,-2,-2) & \Rightarrow G_{\mathrm{F}}=\mathrm{SO}(10) \\
\Phi_{3}: & S \cdot D_{i} \cdot D_{i}=(0,0,0,0,-1,-2,-2,-2,-2) & \Rightarrow G_{\mathrm{F}}=\mathrm{SU}(5) \\
\Phi_{4}: & S \cdot D_{i} \cdot D_{i}=(0,0,0,0,0,-1,-2,-2,-2) & \Rightarrow G_{\mathrm{F}}=\operatorname{SU}(3) \times \mathrm{SU}(2),
\end{array}
$$

which also allows us to read off the flavor symmetries $G_{\mathrm{F}}$ of the strongly coupled SCFT. 
$\operatorname{gdP}_{2}:$ the resolution

$$
\begin{aligned}
& \left\{\left\{x, y, u, u_{1}\right\},\left\{x, y, u_{1}, u_{2}\right\},\left\{y, u_{2}, u_{3}\right\},\left\{y, u_{1}, u_{3}, u_{4}\right\},\left\{y, u_{1}, u_{5}\right\},\left\{u_{1}, u_{3}, u_{6}\right\},\right. \\
& \quad\left\{u_{1}, u_{4}, u_{7}\right\},\left\{u_{3}, u_{4}, u_{10}\right\},\left\{u_{3}, u_{6}, u_{12}\right\},\left\{u_{3}, u_{12}, u_{15}\right\},\left\{u_{3}, v, \delta\right\},\left\{u_{6}, u_{10}, u_{13}\right\} \\
& \left.\quad\left\{u_{1}, u_{5}, u_{8}\right\},\left\{u_{2}, u_{3}, u_{9}\right\},\left\{u_{4}, u_{6}, u_{11}\right\},\left\{u_{10}, u_{12}, u_{14}\right\}\right\}
\end{aligned}
$$

results in a non-flat fiber component with geometry $\mathrm{gdP}_{2}$. The Cartans divisors intersect $S: \delta=0$ as follows

$$
S \cdot D_{i} \cdot D_{i}=(0,0,0,0,0,0,-1,-2,-1),
$$

wherefore the flavor symmetry is $G_{\mathrm{F}}=\mathrm{SU}(2) \times \mathrm{U}(1)$.

$\mathbf{d P}_{\mathbf{1}}$ : the resolution

$$
\begin{aligned}
& \left\{\left\{x, y, u, u_{1}\right\},\left\{x, y, u_{1}, u_{2}\right\},\left\{y, u_{1}, u_{2}, u_{6}\right\},\left\{y, u_{1}, u_{5}\right\},\left\{u_{1}, u_{5}, u_{8}\right\},\left\{y, u_{6}, u_{4}\right\}\right. \\
& \quad\left\{y, u_{2}, u_{3}\right\},\left\{u_{2}, u_{3}, u_{9}\right\},\left\{u_{2}, u_{4}, u_{12}\right\},\left\{u_{3}, u_{4}, u_{10}\right\},\left\{u_{4}, u_{6}, u_{11}\right\},\left\{u_{4}, u_{9}, u_{15}\right\} \\
& \left.\quad\left\{u_{4}, u_{12}, u_{13}\right\},\left\{u_{4}, u_{15}, u_{14}\right\},\left\{u_{1}, u_{4}, u_{7}\right\},\left\{u_{3}, v, \delta\right\}\right\}
\end{aligned}
$$

leads to

$$
S \cdot D_{i} \cdot D_{i}=(0,0,0,0,0,0,0,-1,0) .
$$

Note that $S$ and $D_{8}$ intersects at a rational curve with self-intersection 0 on $S$. The flavor symmetry is $G_{\mathrm{F}}=\mathrm{U}(1)$.

$\operatorname{gdP}_{1} \cong \mathbb{F}_{2}$ : the resolution

$$
\begin{aligned}
& \left\{\left\{x, y, u, u_{1}\right\},\left\{x, y, u_{1}, u_{2}\right\},\left\{y, u_{1}, u_{2}, u_{6}\right\},\left\{y, u_{1}, u_{5}\right\},\left\{y, u_{2}, u_{3}\right\},\left\{u_{1}, u_{5}, u_{8}\right\},\right. \\
& \quad\left\{u_{3}, u_{6}, u_{12}\right\},\left\{y, u_{6}, u_{4}\right\},\left\{u_{4}, u_{6}, u_{11}\right\},\left\{u_{3}, u_{12}, u_{15}\right\},\left\{u_{2}, u_{3}, u_{9}\right\},\left\{u_{4}, u_{12}, u_{13}\right\}, \\
& \left.\quad\left\{y, u_{12}, u_{10}\right\},\left\{u_{10}, u_{12}, u_{14}\right\},\left\{u_{5}, u_{6}, u_{7}\right\},\left\{u_{3}, v, \delta\right\}\right\}
\end{aligned}
$$

results in

$$
S \cdot D_{i} \cdot D_{i}=(0,0,0,0,0,0,0,-2,0) .
$$

Note that $S$ and $D_{6}$ intersects at a rational curve with self-intersection 0 on $S$. The flavor symmetry is $G_{\mathrm{F}}=\mathrm{SU}(2)$.

\section{D.2 Blow-ups for the rank two E-string $\left(\mathrm{E}_{8}, \mathrm{SU}(2)\right)$}

We start with the blow-up that generates the geometry associated to the marginal theory in $5 \mathrm{~d}$. This is the key input for our subsequent analysis, in particular the CFDs. We also provide some example blow-ups, which illustrate some of the salient features of non-flat resolutions in higher rank models.

\section{D.2.1 Geometry for the marginal theory}

To derive the blow-up for the marginal theory, where all of the Dynkin nodes of the affine $\mathrm{E}_{8} \times \mathrm{SU}(2)$ are wrapped $(-2)$-curves, we need to blow up the point $U=V=0$ in the base first: $\left(U, V ; \delta_{1}\right)$. The Weierstrass model then becomes

$$
y^{2}=x^{3}+f_{4} U^{4} V \delta_{1} x+g_{6} U^{5} V^{2} \delta_{1},
$$


while the Tate model is

$$
y^{2}+b_{1} U x y+b_{3} U^{3} V \delta_{1} y=x^{3}+b_{2} U^{2} x^{2}+b_{4} U^{4} V \delta_{1} x+b_{6} U^{5} V^{2} \delta_{1} .
$$

Because of the base blow-up, the locus $U=V=0$ has been removed. In addition, we now apply the resolution sequence

$$
\begin{gathered}
\left\{\left\{x, y, U, u_{1}\right\},\left\{x, y, V, v_{1}\right\},\left\{x, y, u_{1}, u_{2}\right\},\left\{y, u_{2}, u_{3}\right\},\left\{\delta_{1}, u_{3}, \delta_{2}\right\},\left\{y, u_{1}, u_{3}, u_{4}\right\}\right. \\
\left\{y, u_{1}, u_{5}\right\},\left\{u_{1}, u_{3}, u_{6}\right\},\left\{u_{1}, u_{4}, u_{7}\right\},\left\{u_{1}, u_{5}, u_{8}\right\},\left\{u_{2}, u_{3}, u_{9}\right\},\left\{u_{3}, u_{4}, u_{10}\right\} \\
\left.\left\{u_{4}, u_{6}, u_{11}\right\},\left\{u_{3}, u_{6}, u_{12}\right\},\left\{u_{6}, u_{10}, u_{13}\right\},\left\{u_{10}, u_{12}, u_{14}\right\},\left\{u_{3}, u_{12}, u_{15}\right\}\right\}
\end{gathered}
$$

The Cartan divisors $D_{i}^{\mathrm{E} 8}(i=0, \ldots, 8)$ of $\mathrm{E}_{8}$ and $D_{i}^{\mathrm{SU}(2)}$ of $\mathrm{SU}(2)$ are given by the following hypersurface equations (e. g. $U$ means $U=0$ ):

$$
\begin{aligned}
\left(U, u_{8}, u_{7}, u_{11}, u_{13}, u_{14}, u_{15}, u_{9}, u_{10}\right) & \equiv\left(D_{0}^{\mathrm{E}_{8}}, D_{1}^{\mathrm{E}_{8}}, D_{2}^{\mathrm{E}_{8}}, D_{3}^{\mathrm{E}_{8}}, D_{4}^{\mathrm{E}_{8}}, D_{5}^{\mathrm{E}_{8}}, D_{6}^{\mathrm{E}_{8}}, D_{7}^{\mathrm{E}_{8}}, D_{8}^{\mathrm{E}_{8}}\right) \\
\left(V, v_{1}\right) & \equiv\left(D_{0}^{\mathrm{SU}(2)}, D_{1}^{\mathrm{SU}(2)}\right) .
\end{aligned}
$$

The two non-flat fiber components $S_{1}$ and $S_{2}$ are given by the hypersurface equations $\delta_{1}=0$ and $\delta_{2}=0$ respectively. The full intersection matrices involving the two non-flat surface components are

\begin{tabular}{c|ccccccccccccc}
$S_{1}$ & $D_{0}^{\mathrm{E}_{8}}$ & $D_{1}^{\mathrm{E}_{8}}$ & $D_{2}^{\mathrm{E}_{8}}$ & $D_{3}^{\mathrm{E}_{8}}$ & $D_{4}^{\mathrm{E}_{8}}$ & $D_{5}^{\mathrm{E}_{8}}$ & $D_{6}^{\mathrm{E}_{8}}$ & $D_{7}^{\mathrm{E}_{8}}$ & $D_{8}^{\mathrm{E}_{8}}$ & $D_{0}^{\mathrm{SU}(2)}$ & $D_{1}^{\mathrm{SU}(2)}$ & $S_{1}$ & $S_{2}$ \\
\hline$D_{0}^{\mathrm{E}_{8}}$ & -2 & 1 & 0 & 0 & 0 & 0 & 0 & 0 & 0 & 0 & 0 & 0 & 0 \\
$D_{1}^{\mathrm{E}_{8}}$ & 1 & -1 & 0 & 0 & 0 & 0 & 0 & 0 & 0 & 0 & 0 & -1 & 1 \\
$D_{2}^{\mathrm{E}_{8}}$ & 0 & 0 & 0 & 0 & 0 & 0 & 0 & 0 & 0 & 0 & 0 & 0 & 0 \\
$D_{3}^{\mathrm{E}_{8}}$ & 0 & 0 & 0 & 0 & 0 & 0 & 0 & 0 & 0 & 0 & 0 & 0 & 0 \\
$D_{4}^{\mathrm{E}_{8}}$ & 0 & 0 & 0 & 0 & 0 & 0 & 0 & 0 & 0 & 0 & 0 & 0 & 0 \\
$D_{5}^{\mathrm{E}_{8}}$ & 0 & 0 & 0 & 0 & 0 & 0 & 0 & 0 & 0 & 0 & 0 & 0 & 0 \\
$D_{6}^{\mathrm{E}_{8}}$ & 0 & 0 & 0 & 0 & 0 & 0 & 0 & 0 & 0 & 0 & 0 & 0 & 0 \\
$D_{7}^{\mathrm{E}_{8}}$ & 0 & 0 & 0 & 0 & 0 & 0 & 0 & 0 & 0 & 0 & 0 & 0 & 0 \\
$D_{8}^{\mathrm{E}_{8}}$ & 0 & 0 & 0 & 0 & 0 & 0 & 0 & 0 & 0 & 0 & 0 & 0 & 0 \\
$D_{0}^{\mathrm{SU}(2)}$ & 0 & 0 & 0 & 0 & 0 & 0 & 0 & 0 & 0 & -2 & 2 & 0 & 0 \\
$D_{1}^{\mathrm{SU}(2)}$ & 0 & 0 & 0 & 0 & 0 & 0 & 0 & 0 & 0 & 2 & -2 & 0 & 0 \\
$S_{1}$ & 0 & -1 & 0 & 0 & 0 & 0 & 0 & 0 & 0 & 0 & 0 & -2 & 2 \\
$S_{2}$ & 0 & 1 & 0 & 0 & 0 & 0 & 0 & 0 & 0 & 0 & 0 & 2 & -2
\end{tabular}

\begin{tabular}{c|ccccccccccccc}
$S_{2}$ & $D_{0}^{\mathrm{E} 8}$ & $D_{1}^{\mathrm{E} 8}$ & $D_{2}^{\mathrm{E} 8}$ & $D_{3}^{\mathrm{E} 8}$ & $D_{4}^{\mathrm{E} 8}$ & $D_{5}^{\mathrm{E} 8}$ & $D_{6}^{\mathrm{E} 8}$ & $D_{7}^{\mathrm{E} 8}$ & $D_{8}^{\mathrm{E} 8}$ & $D_{0}^{\mathrm{SU}(2)}$ & $D_{1}^{\mathrm{SU}(2)}$ & $S_{1}$ & $S_{2}$ \\
\hline$D_{0}^{\mathrm{E}_{8}}$ & 0 & 0 & 0 & 0 & 0 & 0 & 0 & 0 & 0 & 0 & 0 & 0 & 0 \\
$D_{1}^{\mathrm{E}_{8}}$ & 0 & -1 & 1 & 0 & 0 & 0 & 0 & 0 & 0 & 0 & 0 & 1 & -1 \\
$D_{2}^{\mathrm{E}_{8}}$ & 0 & 1 & -2 & 1 & 0 & 0 & 0 & 0 & 0 & 0 & 0 & 0 & 0 \\
$D_{3}^{\mathrm{E}_{8}}$ & 0 & 0 & 1 & -2 & 1 & 0 & 0 & 0 & 0 & 0 & 0 & 0 & 0 \\
$D_{4}^{\mathrm{E}_{8}}$ & 0 & 0 & 0 & 1 & -2 & 1 & 0 & 0 & 0 & 0 & 0 & 0 & 0 \\
$D_{5}^{\mathrm{E}_{8}}$ & 0 & 0 & 0 & 0 & 1 & -2 & 1 & 1 & 0 & 0 & 0 & 0 & 0 \\
$D_{6}^{\mathrm{E}_{8}}$ & 0 & 0 & 0 & 0 & 0 & 1 & -2 & 0 & 0 & 0 & 0 & 0 & 0 \\
$D_{7}^{\mathrm{E} 8}$ & 0 & 0 & 0 & 0 & 0 & 1 & 0 & -2 & 1 & 0 & 0 & 0 & 0 \\
$D_{8}^{\mathrm{E} 8}$ & 0 & 0 & 0 & 0 & 0 & 0 & 0 & 1 & -2 & 0 & 0 & 0 & 0 \\
$D_{0}^{\mathrm{SU}(2)}$ & 0 & 0 & 0 & 0 & 0 & 0 & 0 & 0 & 0 & 0 & 0 & 0 & 0 \\
$D_{1}^{\mathrm{SU}(2)}$ & 0 & 0 & 0 & 0 & 0 & 0 & 0 & 0 & 0 & 0 & 0 & 0 & 0 \\
$S_{1}$ & 0 & 1 & 0 & 0 & 0 & 0 & 0 & 0 & 0 & 0 & 0 & 2 & -2 \\
$S_{2}$ & 0 & -1 & 0 & 0 & 0 & 0 & 0 & 0 & 0 & 0 & 0 & -2 & 2
\end{tabular}


We can also collect all the triple intersection numbers $S_{i} \cdot D_{j}^{2}$ in the following reduced intersection matrix:

$$
\begin{array}{r|lll|ll|ll}
D= & D_{0}^{\mathrm{E}} & \cdots & D_{8}^{\mathrm{E}} & D_{0}^{\mathrm{SU}(2)} & D_{1}^{\mathrm{SU}(2)} & S_{1} & S_{2} \\
\hline S_{1} \cdot D_{i} \cdot D_{i} & & & & & & \\
S_{2} \cdot D_{i} \cdot D_{i} & & & & & &
\end{array}
$$

From this we can compute the genus of the curve along which the two sections $S_{i}$ intersect,

$$
2 g-2=S_{1}^{2} S_{2}+S_{2}^{2} S_{1} .
$$

For the genus $g=0$ case, we can read off the number of flavors $N_{f}$ in the $\mathrm{SU}(3)$ gauge description and the number of mass deformations $M=N_{f}+1$, as in (B.7):

$$
M=17-\left(S_{1}^{3}+S_{2}^{3}\right) .
$$

For higher genus cases, we can use (B.16) with $N_{a}=g$ and $a_{a}=0$ if an $\operatorname{Sp}(2)$ gauge description exists:

$$
\begin{gathered}
N_{f}=16-8 g-\left(S_{1}^{3}+S_{2}^{3}\right) \\
M=17-7 g-\left(S_{1}^{3}+S_{2}^{3}\right),
\end{gathered}
$$

where $N_{f}$ is the number of fundamental flavors in the $\operatorname{Sp}(2)+g \mathbf{A S}+N_{f} \mathbf{F}$ gauge theory.

In our example, the reduced intersection matrix is

\begin{tabular}{c|ccccccccc|cc|cc}
$S_{i} \cdot D_{j}^{2}$ & $D_{0}^{\mathrm{E}_{8}}$ & $D_{1}^{\mathrm{E}_{8}}$ & $D_{2}^{\mathrm{E}_{8}}$ & $D_{3}^{\mathrm{E}_{8}}$ & $D_{4}^{\mathrm{E}_{8}}$ & $D_{5}^{\mathrm{E}_{8}}$ & $D_{6}^{\mathrm{E}_{8}}$ & $D_{7}^{\mathrm{E}_{8}}$ & $D_{8}^{\mathrm{E}_{8}}$ & $D_{0}^{\mathrm{SU}(2)}$ & $D_{1}^{\mathrm{SU}(2)}$ & $S_{1}$ & $S_{2}$ \\
\hline$S_{1}$ & -2 & -1 & 0 & 0 & 0 & 0 & 0 & 0 & 0 & -2 & -2 & -2 & -2 \\
$S_{2}$ & 0 & -1 & -2 & -2 & -2 & -2 & -2 & -2 & -2 & 0 & 0 & 2 & 2
\end{tabular},

from which we can see that the intersection curve $C=S_{1} \cdot S_{2}$ is a genus one curve rather than a rational curve. We draw the configuration of curves on $S_{1}$ and $S_{2}$ in figure 21 . In fact, the surface $S_{1}$ is a ruled surface over the genus one curve $C$. From the matrix elements $S_{1}^{3}$ and $S_{2}^{3}$, we can read off $M=10$ from (D.22).

There exists a geometric transition from this configuration into a combination of two rational surfaces, see section 3.5 of [6]. We take the limit in the complex structure moduli space of $S_{1}$, where $C$ becomes a pinched $S^{2}$ with a double point singularity. After we blow up this double point singularity, the surface $S_{1}$ will become a blow-up of Hirzebruch surface $S_{1}^{\prime}=\operatorname{blp}_{2} \mathbb{F}_{m}$ and the surface $S_{2}$ will be blown up into $S_{2}^{\prime}=\operatorname{blp} S_{2}$. The blow-up of $S_{2}$ occurs at a double point $P \subset C \subset S_{2}$. Denoting the exceptional divisor from this blow-up by $e_{P}$, the proper transform of $C$ on $S_{2}^{\prime}$ is then $C_{S_{2}^{\prime}}^{\prime}=C_{S_{2}}-2 e_{P}$, which is a rational curve. The self-intersection of $C^{\prime}=S_{1}^{\prime} \cdot S_{2}^{\prime}$ inside $S_{1}^{\prime}$ can then be computed by

$$
C^{\prime} \cdot S_{1}^{\prime} C^{\prime}=-2-C^{\prime} \cdot S_{2}^{\prime} C^{\prime}=2-C \cdot S_{2} C .
$$

In figure 21, the genus one curve $C$ has self-intersection $(-2)$ on $S_{1}$ and 2 on $S_{2}$. On $S_{2}$, its representation with the standard Picard group of rational surfaces is

$$
C_{S_{2}}=3 h-\sum_{i=1}^{7} e_{i}
$$



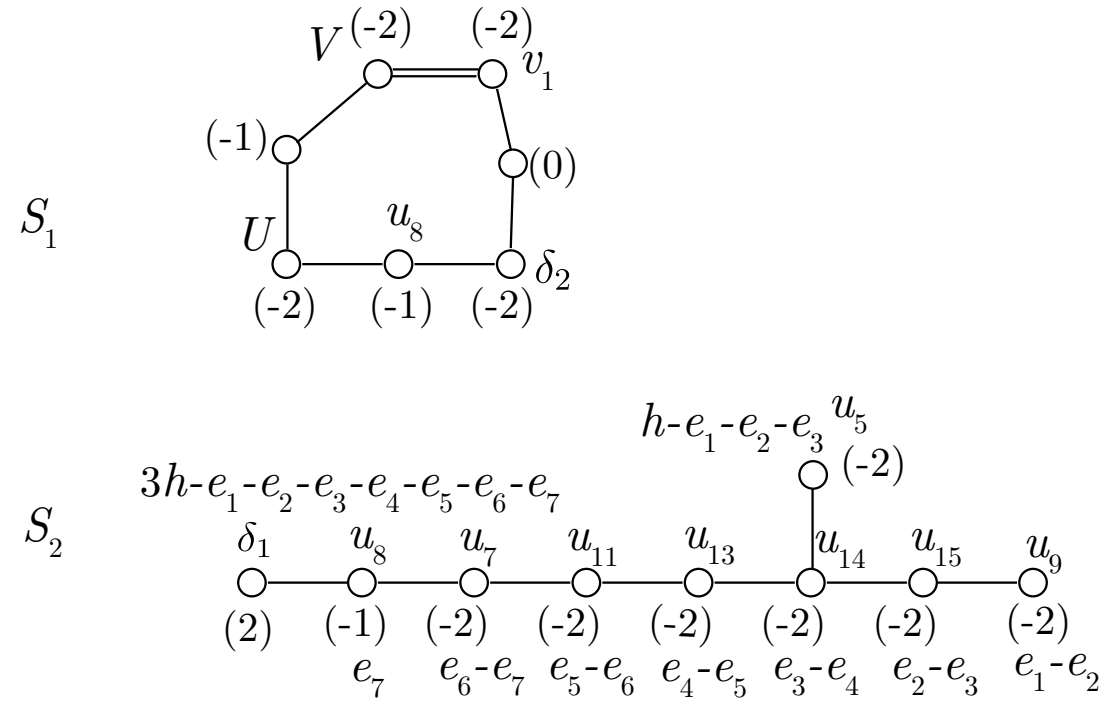

Figure 21. The configuration of curves on the two non-flat fiber components $S_{1}$ and $S_{2}$ in the geometry D.23 before the flop. The number in the bracket denotes the self-intersection number of the curve. The letter denotes an intersection curve with the corresponding divisor in the resolved Calabi-Yau threefold. On $S_{2}$, we also labeled the curves with the generators of the Picard group on a rational surface (C.1). Here $S_{1}$ is not a rational surface.

After the geometric transition, $S_{1}^{\prime}$ becomes the blow-up of a Hirzebruch surface and $S_{2}^{\prime}=\operatorname{gdP}_{8}$. The transformed curve $C_{S_{2}^{\prime}}^{\prime}$ is

$$
C_{S_{2}^{\prime}}^{\prime}=3 h-\sum_{i=1}^{7} e_{i}-2 e_{8}
$$

which is a rational $(-2)$-curve (here $\left.e_{8} \equiv e_{p}\right)$.

On $S_{1}^{\prime}$, from (D.24) we have $C_{S_{1}^{\prime}}^{\prime 2}=0$. The surface $S_{1}^{\prime}$ is a $\mathrm{gdP}_{3}$ after the flop, and the curve $D_{1}^{\mathrm{SU}(2)} \cdot S_{1}^{\prime}$ is a 0 -curve on $S_{1}^{\prime}$. Hence the rational (-2)-curve on $S_{1}$ should have been flopped into $S_{2}^{\prime}$. On $S_{2}^{\prime}=\mathrm{gdP}_{8}$, however, there is no room for a new (-2)-curve, and $D_{1}^{\mathrm{SU}(2)} \cdot S_{2}^{\prime}$ is a $(-1)$-curve on $S_{2}^{\prime}$ instead, see figure 22 . The reduced intersection matrix after the flop is

$$
\begin{array}{c|ccccccccc|cc|cc}
S_{i} \cdot D_{j}^{2} & D_{0}^{\mathrm{E}_{8}} & D_{1}^{\mathrm{E}_{8}} & D_{2}^{\mathrm{E}_{8}} & D_{3}^{\mathrm{E}_{8}} & D_{4}^{\mathrm{E}_{8}} & D_{5}^{\mathrm{E}_{8}} & D_{6}^{\mathrm{E}_{8}} & D_{7}^{\mathrm{E}_{8}} & D_{8}^{\mathrm{E}_{8}} & D_{0}^{\mathrm{SU}(2)} & D_{1}^{\mathrm{SU}(2)} & S_{1} & S_{2} \\
\hline S_{1} & -2 & -1 & 0 & 0 & 0 & 0 & 0 & 0 & 0 & -2 & 0 & 6 & 0 \\
S_{2} & 0 & -1 & -2 & -2 & -2 & -2 & -2 & -2 & -2 & 0 & -1 & -2 & 1
\end{array} .
$$

Note that the Cartan divisor $D_{1}^{\mathrm{SU}(2)}$ is still considered as fully wrapped inside the non-flat fiber, as we assign a weight 2 to the divisors $D_{i}^{\mathrm{SU}(2)}$ on the surface component $S_{2}^{\prime}$. Here we can confirm that the curve $D_{1}^{\mathrm{SU}(2)} \cdot\left(S_{1}^{\prime}+2 S_{2}^{\prime}\right)$ is indeed a rational curve with normal bundle $\mathcal{O}(0)+\mathcal{O}(-2)$ in the Calabi-Yau threefold $Y$. The relevant triple intersection numbers can be read off from figure 22 :

$$
\begin{array}{lr}
D_{1}^{\mathrm{SU}(2) 2} \cdot S_{1}^{\prime}=0, & D_{1}^{\mathrm{SU}(2)} \cdot S_{1}^{\prime 2}=-4 \\
D_{1}^{\mathrm{SU}(2) 2} \cdot S_{2}^{\prime}=D_{1}^{\mathrm{SU}(2)} \cdot S_{2}^{\prime 2}=-1, & D_{1}^{\mathrm{SU}(2)} \cdot S_{1}^{\prime} \cdot S_{2}^{\prime}=2 .
\end{array}
$$



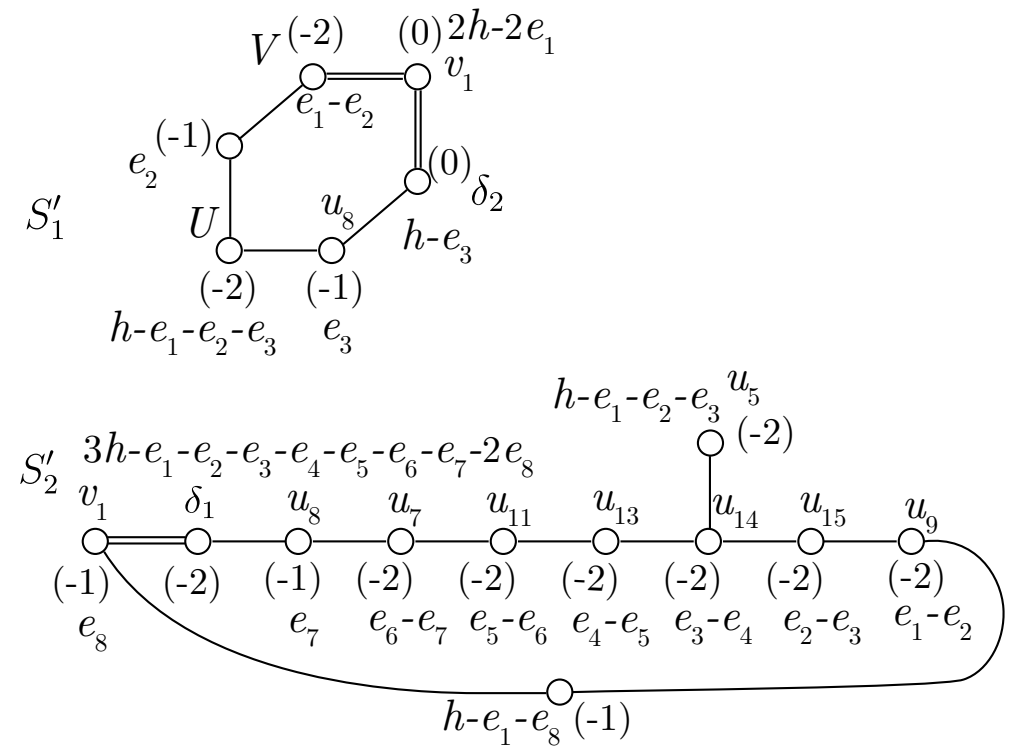

Figure 22. The configuration of curves on $S_{1}^{\prime}$ and $S_{2}^{\prime}$ in the $\left(\mathrm{E}_{8}, \mathrm{SU}(2)\right)$ marginal geometry after the flop. The number in the bracket denotes the self-intersection number of the curve. The letter denotes an intersection curve with the corresponding divisor in the resolved Calabi-Yau threefold.

Hence we can compute

$$
\begin{aligned}
& D_{1}^{\mathrm{SU}(2) 2} \cdot\left(S_{1}^{\prime}+2 S_{2}^{\prime}\right)=-2 \\
& D_{1}^{\mathrm{SU}(2)} \cdot\left(S_{1}^{\prime}+2 S_{2}^{\prime}\right)^{2}=0,
\end{aligned}
$$

which indeed tells us $D_{1}^{\mathrm{SU}(2)} \cdot\left(S_{1}^{\prime}+2 S_{2}^{\prime}\right)$ has the normal bundle $\mathcal{O}(0)+\mathcal{O}(-2)$.

If we contract the $(-1)$-curve $h-e_{1}-e_{8}$ on $S_{2}^{\prime}$. Then the curve $D_{1}^{\mathrm{SU}(2)} \cdot S_{2}^{\prime}$ on $S_{2}^{\prime}$ becomes a rational curve with self-intersection 0 , which is also reflected in the CFD tree in figure 12, see the one with flavor symmetry $\mathrm{SO}(16) \times \mathrm{SU}(2)$ for example.

Similarly, in any blow down of the figure 22 , the divisor $D_{1}^{\mathrm{SU}(2)}$ has to be considered as fully wrapped and generate the flavor symmetry if $D_{1}^{\mathrm{SU}(2) 2} \cdot S_{2}=-1$.

\section{D.2.2 Example resolutions: $\left(\mathrm{E}_{8}, \mathrm{SU}(2)\right)$}

Besides the geometry of marginal theory, the distinct blow-ups are characterized in terms of different orders of resolution of the $U$ and $V$ (and blow-ups thereof). We will provide here a few example resolutions of the rank two E-string Weierstrass model that correspond to descendant 5D SCFTs of the marginal theory.

Consider the first blow-up (we again use the notation for blow-ups introduced in section 2.5.3)

$$
\begin{gathered}
\mathrm{BU}_{1}^{\left(\mathrm{E}_{8}, \mathrm{SU}(2)\right)}= \\
\left\{\left\{x, y, U, u_{1}\right\},\left\{x, y, V, v_{1}\right\},\left\{x, y, u_{1}, u_{2}\right\},\left\{y, u_{2}, u_{3}\right\},\left\{y, u_{1}, v_{1}, \delta_{1}\right\},\left\{y, u_{1}, u_{3}, u_{4}\right\}\right. \\
\quad\left\{y, u_{1}, u_{5}\right\},\left\{u_{1}, u_{3}, u_{6}\right\},\left\{u_{1}, u_{4}, u_{7}\right\},\left\{u_{1}, u_{5}, u_{8}\right\},\left\{u_{2}, u_{3}, u_{9}\right\},\left\{u_{3}, u_{4}, u_{10}\right\} \\
\quad\left\{u_{4}, u_{6}, u_{11}\right\},\left\{u_{3}, u_{6}, u_{12}\right\},\left\{u_{6}, u_{10}, u_{13}\right\},\left\{u_{10}, u_{12}, u_{14}\right\},\left\{u_{3}, u_{12}, u_{15}\right\} \\
\left.\quad\left\{\delta_{1}, u_{3}, \delta_{2}\right\}\right\} .
\end{gathered}
$$


The data that we need to read off from the this resolution are the triple intersection number of the non-flat surface components of the codimension two fibers $S_{i}$ with the Cartans of both $\mathrm{E}_{8}$ and $\mathrm{SU}(2)$, respectively

\begin{tabular}{|c|c|c|c|c|c|c|c|c|c|c|c|c|c|}
\hline$S_{1}$ & $D_{0}^{\mathrm{E}_{8}}$ & $D_{1}^{\mathrm{E}_{8}}$ & $D_{2}^{\mathrm{E}_{8}}$ & $D_{3}^{\mathrm{E}_{8}}$ & $D_{4}^{\mathrm{E}_{8}}$ & $D_{5}^{\mathrm{E}_{8}}$ & $D_{6}^{\mathrm{E}_{8}}$ & $D_{7}^{\mathrm{E}_{8}}$ & $D_{8}^{\mathrm{E}_{8}}$ & $D_{0}^{\mathrm{SU}(2)}$ & $D_{1}^{\mathrm{SU}(2)}$ & $S_{1}$ & $S_{2}$ \\
\hline$D_{0}^{\mathrm{E}_{8}}$ & 0 & 0 & 0 & 0 & 0 & 0 & 0 & 0 & 0 & 0 & 0 & 0 & 0 \\
\hline$D_{1}^{\mathrm{E}_{8}}$ & 0 & -1 & 1 & 0 & 0 & 0 & 0 & 0 & 0 & 1 & 0 & -1 & 0 \\
\hline$D_{2}^{\mathrm{E}_{8}}$ & 0 & 1 & -2 & 1 & 0 & 0 & 0 & 0 & 0 & 0 & 0 & 0 & 0 \\
\hline$D_{3}^{\mathrm{E}_{8}}$ & 0 & 0 & 1 & -2 & 1 & 0 & 0 & 0 & 0 & 0 & 0 & 0 & 0 \\
\hline$D_{4}^{\mathrm{E} 8}$ & 0 & 0 & 0 & 1 & -2 & 1 & 0 & 0 & 0 & 0 & 0 & 0 & 0 \\
\hline$D_{5}^{\mathrm{E}_{8}}$ & 0 & 0 & 0 & 0 & 1 & -1 & 0 & 0 & 0 & 0 & 0 & -1 & 1 \\
\hline$D_{6}^{\mathrm{E}_{8}}$ & 0 & 0 & 0 & 0 & 0 & 0 & 0 & 0 & 0 & 0 & 0 & 0 & 0 \\
\hline$D_{7}^{\mathrm{E} 8}$ & 0 & 0 & 0 & 0 & 0 & 0 & 0 & 0 & 0 & 0 & 0 & 0 & 0 \\
\hline$D_{8}^{\mathrm{E}_{8}}$ & 0 & 0 & 0 & 0 & 0 & 0 & 0 & 0 & 0 & 0 & 0 & 0 & 0 \\
\hline$D_{0}^{\mathrm{SU}(2)}$ & 0 & 1 & 0 & 0 & 0 & 0 & 0 & 0 & 0 & 0 & 2 & -2 & 0 \\
\hline$D_{1}^{\mathrm{SU}(2)}$ & 0 & 0 & 0 & 0 & 0 & 0 & 0 & 0 & 0 & 2 & 0 & -4 & 2 \\
\hline$S_{1}$ & 0 & -1 & 0 & 0 & 0 & -1 & 0 & 0 & 0 & -2 & -4 & 4 & 2 \\
\hline$S_{2}$ & 0 & 0 & 0 & 0 & 0 & 1 & 0 & 0 & 0 & 0 & 2 & 2 & -4 \\
\hline$S_{2}$ & $D_{0}^{\mathrm{E}_{8}}$ & $D_{1}^{\mathrm{E}_{8}}$ & $D_{2}^{\mathrm{E}_{8}}$ & $D_{3}^{\mathrm{E}_{8}}$ & $D_{4}^{\mathrm{E}_{8}}$ & $D_{5}^{\mathrm{E}_{8}}$ & $D_{6}^{\mathrm{E}_{8}}$ & $D_{7}^{\mathrm{E}_{8}}$ & $D_{8}^{\mathrm{E}_{8}}$ & $D_{0}^{\mathrm{SU}(2)}$ & $D_{1}^{\mathrm{SU}(2)}$ & $S_{1}$ & $S_{2}$ \\
\hline$D_{0}^{\mathrm{E}_{8}}$ & 0 & 0 & 0 & 0 & 0 & 0 & 0 & 0 & 0 & 0 & 0 & 0 & 0 \\
\hline$D_{1}^{\mathrm{E}_{8}}$ & 0 & 0 & 0 & 0 & 0 & 0 & 0 & 0 & 0 & 0 & 0 & 0 & 0 \\
\hline$D_{2}^{\mathrm{E} 8}$ & 0 & 0 & 0 & 0 & 0 & 0 & 0 & 0 & 0 & 0 & 0 & 0 & 0 \\
\hline$D_{3}^{\mathrm{E}_{8}}$ & 0 & 0 & 0 & 0 & 0 & 0 & 0 & 0 & 0 & 0 & 0 & 0 & 0 \\
\hline$D_{4}^{\mathrm{E}_{8}}$ & 0 & 0 & 0 & 0 & 0 & 0 & 0 & 0 & 0 & 0 & 0 & 0 & 0 \\
\hline$D_{5}^{\mathrm{E}_{8}}$ & 0 & 0 & 0 & 0 & 0 & -1 & 1 & 0 & 1 & 0 & 0 & 1 & -1 \\
\hline$D_{6}^{\mathrm{E}_{8}}$ & 0 & 0 & 0 & 0 & 0 & 1 & \begin{tabular}{|l|}
-1 \\
\end{tabular} & 0 & 0 & 0 & 1 & 0 & -1 \\
\hline$D_{7}^{\mathrm{E}_{8}}$ & 0 & 0 & 0 & 0 & 0 & 0 & 0 & 0 & 0 & 0 & 0 & 0 & 0 \\
\hline$D_{8}^{\mathrm{E}_{8}}$ & 0 & 0 & 0 & 0 & 0 & 1 & 0 & 0 & \begin{tabular}{|l|}
-2 \\
\end{tabular} & 0 & 0 & 0 & 0 \\
\hline$D_{0}^{\mathrm{SU}(2)}$ & 0 & 0 & 0 & 0 & 0 & 0 & 0 & 0 & 0 & 0 & 0 & 0 & 0 \\
\hline$D_{1}^{\mathrm{SU}(2)}$ & 0 & 0 & 0 & 0 & 0 & 0 & 1 & 0 & 0 & 0 & 11 & 2 & -3 \\
\hline$S_{1}$ & 0 & 0 & 0 & 0 & 0 & 1 & 0 & 0 & 0 & 0 & 2 & 2 & -4 \\
\hline$S_{2}$ & 0 & 0 & 0 & 0 & 0 & -1 & -1 & 0 & 0 & 0 & -3 & -4 & 7 \\
\hline
\end{tabular}

From these intersection matrices we can read off the curves $F_{j}^{\mathrm{E}_{8}}$ (fibral curves of the Cartan divisors) that are contained also in the surface components $S_{i}$ (see the entries that are boxed in the above intersection matrices). If again $D_{j}^{\mathrm{E}_{8} 2} \cdot S_{i}=-2$ then the curve $F_{j}^{\mathrm{E}_{8}}$ is fully contained in the surface $S_{i}$, if this is -1 , then the curve $F_{j}$ splits in codimension two and one of the split components are contained in $S_{i}$. In the above case, the curves $F_{j}^{\mathrm{E}_{8}}$ are entirely contained in the surfaces for $j=2,3,4,5,8$, and $F_{j}^{\mathrm{E}_{8}}$ for $j=1,6$, the curves split and one of the irreducible components are contained. Note that none of the $F_{j}^{\mathrm{SU}(2)}$ are contained in the surface components in this case. For $j=5$ note that the curve splits, but both irreducible -1 curves are contained in either one of the surfaces $S_{i}$, so that in the singular, conformal field theory limit, the curve $F_{5}$ will shrink to zero size and contribute to the flavor symmetry. 
For the blow-up $\mathrm{BU}_{1}^{\left(\mathrm{E}_{8}, \mathrm{SU}(2)\right)}$, we have the reduced intersection matrix

$$
\begin{aligned}
& \mathrm{BU}_{1}^{\left(\mathrm{E}_{8}, \mathrm{SU}(2)\right)}: \\
& \begin{array}{r|ccccccccc|cc|cc}
S_{i} \cdot D_{j}^{2} & D_{0} & D_{1} & D_{2} & D_{3} & D_{4} & D_{5} & D_{6} & D_{7} & D_{8} & D_{0}^{\mathrm{SU}(2)} & D_{1}^{\mathrm{SU}(2)} & S_{1} & S_{2} \\
\hline S_{1} & 0 & -1 & -2 & -2 & -2 & -1 & 0 & 0 & 0 & 0 & 0 & 4 & -4 \\
S_{2} & 0 & 0 & 0 & 0 & 0 & -1 & -1 & 0 & -2 & 0 & 1 & 2 & 7 \\
\hline n\left(F_{j}\right) & 0 & -1 & -2 & -2 & -2 & -2 & -1 & 0 & -2 & 0 & 2 & - & -
\end{array}
\end{aligned}
$$

In the last line we added the integer $n\left(F_{j}\right)$ defined in (3.4), which determines the nonabelian part of the strongly coupled flavor symmetry. From (D.32) and (B.7), we can compute

$$
G_{F, n a}=\mathrm{SU}(6), g=0, M=6, k=\frac{3}{2}, a=1 .
$$

The geometry is shown in figure 7 .

We can read off the codimension-two fiber in this case, including how the irreducible fiber components of the codimension one fibers split as well as the wrapping by the non-flat fiber components $S_{i}$. For the blow-up $\mathrm{BU}_{1}^{\left(\mathrm{E}_{8}, \mathrm{SU}(2)\right)}$ in (D.30), the codimension two loci are given by all pair-wise intersections of

$$
D_{i}^{\mathrm{E} 8} \cdot D_{k}^{\mathrm{SU}(2)}, \quad D_{i}^{\mathrm{E} 8} \cdot S_{j}, \quad j=1,2 .
$$

From these intersections, we can determine how the $\mathrm{E}_{8}$ fiber components split. In the example (D.30) the irreducible components are listed in the following table, including the information, indicated by a dash, in which codimension two divisor they are contained in $-S_{j}$ or $D_{i}^{\mathrm{SU}(2)}$ :

\begin{tabular}{|c|c|c|c|c|c|c|}
\hline $\mathrm{E}_{8}$ Root & Irreducible Components & $C \cdot C$ & $S_{1}$ & $S_{2}$ & $D_{0}^{\mathrm{SU}(2)}$ & $D_{1}^{\mathrm{SU}(2)}$ \\
\hline$\alpha_{0}^{\mathrm{E}_{8}}$ & $D_{0}^{\mathrm{E}_{8}} \cdot D_{0}^{\mathrm{SU}(2)}$ & -2 & & & - & \\
\hline$\alpha_{1}^{\mathrm{E}_{8}}$ & $D_{1}^{\mathrm{E}_{8}} \cdot D_{0}^{\mathrm{SU}(2)}$ & -1 & & & - & \\
& $D_{1}^{\mathrm{E}_{8}} \cdot S_{1}$ & -1 & - & & & \\
\hline$\alpha_{2}^{\mathrm{E}_{8}}$ & $D_{2}^{\mathrm{E}_{8}} \cdot S_{1}$ & -2 & - & & & \\
\hline$\alpha_{3}^{\mathrm{E}_{8}}$ & $D_{3}^{\mathrm{E}_{8}} \cdot S_{1}$ & -2 & - & & & \\
\hline$\alpha_{4}^{\mathrm{E}_{8}}$ & $D_{4}^{\mathrm{E}_{8}} \cdot S_{1}$ & -2 & - & & & \\
\hline$\alpha_{5}^{\mathrm{E}_{8}}$ & $D_{5}^{\mathrm{E}_{8}} \cdot S_{1}$ & -1 & - & & & \\
\hline$\alpha_{6}^{\mathrm{E}_{8}}$ & $D_{5}^{\mathrm{E}_{8}} \cdot S_{2}$ & -1 & & - & & \\
\hline$\alpha_{7}^{\mathrm{E}_{8}}$ & $D_{6}^{\mathrm{E}_{8}} \cdot S_{2}$ & -1 & & - & & \\
\hline$\alpha_{8}^{\mathrm{E}_{8}}$ & $D_{6}^{\mathrm{E}_{8}} \cdot D_{1}^{\mathrm{SU}(2)}$ & -1 & & & & - \\
\hline
\end{tabular}

Including the information about the relative intersections of these, which follow almost automatically from the above table, we can read off the codimension two fiber in figure 7 . 
To count the number of abelian flavor symmetry factors $\mathrm{U}(1)^{s}$, we compute the number of linearly independent curves in form of $D_{i}^{\mathrm{E}_{8}} \cdot S_{j}$ and $D_{i}^{\mathrm{SU}(2)} \cdot S_{j}$, which is the eight given by the rank of the following intersection matrix:

\begin{tabular}{c|ccccccccc|cc} 
& $D_{0}^{\mathrm{E}_{8}}$ & $D_{1}^{\mathrm{E}_{8}}$ & $D_{2}^{\mathrm{E}_{8}}$ & $D_{3}^{\mathrm{E}_{8}}$ & $D_{4}^{\mathrm{E}_{8}}$ & $D_{5}^{\mathrm{E}_{8}}$ & $D_{6}^{\mathrm{E}_{8}}$ & $D_{7}^{\mathrm{E}_{8}}$ & $D_{8}^{\mathrm{E}_{8}}$ & $D_{0}^{\mathrm{SU}(2)}$ & $D_{1}^{\mathrm{SU}(2)}$ \\
\hline$D_{1}^{\mathrm{E} 8} \cdot S_{1}$ & 0 & -1 & 1 & 0 & 0 & 0 & 0 & 0 & 0 & 1 & 0 \\
$D_{2}^{\mathrm{E} 8} \cdot S_{1}$ & 0 & 1 & -2 & 1 & 0 & 0 & 0 & 0 & 0 & 0 & 0 \\
$D_{3}^{\mathrm{E}_{8}} \cdot S_{1}$ & 0 & 0 & 1 & -2 & 1 & 0 & 0 & 0 & 0 & 0 & 0 \\
$D_{4}^{\mathrm{E}_{8}} \cdot S_{1}$ & 0 & 0 & 0 & 1 & -2 & 1 & 0 & 0 & 0 & 0 & 0 \\
$D_{5}^{\mathrm{E}_{8}} \cdot S_{1}$ & 0 & 0 & 0 & 0 & 1 & -1 & 0 & 0 & 0 & 0 & 0 \\
$D_{5}^{\mathrm{E}_{8}} \cdot S_{2}$ & 0 & 0 & 0 & 0 & 0 & -1 & 1 & 0 & 1 & 0 & 0 \\
$D_{6}^{\mathrm{E}_{8}} \cdot S_{2}$ & 0 & 0 & 0 & 0 & 0 & 1 & -1 & 0 & 0 & 0 & 1 \\
$D_{8}^{\mathrm{E}_{8}} \cdot S_{2}$ & 0 & 0 & 0 & 0 & 0 & 1 & 0 & 0 & -2 & 0 & 0 \\
$D_{0}^{\mathrm{SU}(2)} \cdot S_{1}$ & 0 & 1 & 0 & 0 & 0 & 0 & 0 & 0 & 0 & 0 & 2 \\
$D_{1}^{\mathrm{SU}(2)} \cdot S_{1}$ & 0 & 0 & 0 & 0 & 0 & 0 & 0 & 0 & 0 & 2 & 0 \\
$D_{1}^{\mathrm{SU}(2)} \cdot S_{2}$ & 0 & 0 & 0 & 0 & 0 & 0 & 1 & 0 & 0 & 0 & 1
\end{tabular}

Substracting eight with the rank of gauge group $\mathrm{SU}(3)_{G}$, we get the total rank of $\operatorname{rk}\left(G_{\mathrm{F}}\right)=$ 6 . Hence we conclude that the number of abelian flavor symmetry factors $s=1$ in this case.

In conclusion, the total flavor symmetry of this SCFT is

$$
G_{\mathrm{F}}=\mathrm{SU}(6) \times \mathrm{U}(1)
$$

A model that is closely related to $\mathrm{BU}_{1}^{\left(\mathrm{E}_{8}, \mathrm{SU}(2)\right)}$ is the following ${ }^{14}$

$$
\begin{aligned}
& \mathrm{BU}_{2}^{\left(\mathrm{E}_{8}, \mathrm{SU}(2)\right)}= \\
& \left\{\left\{x, y, U, u_{1}\right\},\left\{x, y, V, v_{1}\right\},\left\{x, y, u_{1}, u_{2}\right\},\left\{y, u_{2}, u_{3}\right\},\left\{y, u_{1}, v_{1}, \delta_{1}\right\},\left\{\delta_{1}, u_{3}, \delta_{2}\right\}\right. \\
& \quad\left\{y, u_{1}, u_{3}, u_{4}\right\},\left\{y, u_{1}, u_{5}\right\},\left\{u_{1}, u_{3}, u_{6}\right\},\left\{u_{1}, u_{4}, u_{7}\right\},\left\{u_{1}, u_{5}, u_{8}\right\},\left\{u_{2}, u_{3}, u_{9}\right\} \\
& \quad\left\{u_{3}, u_{4}, u_{10}\right\},\left\{u_{4}, u_{6}, u_{11}\right\},\left\{u_{3}, u_{6}, u_{12}\right\},\left\{u_{6}, u_{10}, u_{13}\right\},\left\{u_{10}, u_{12}, u_{14}\right\} \\
& \left.\quad\left\{u_{3}, u_{12}, u_{15}\right\}\right\}
\end{aligned}
$$

The reduced triple intersection matrix is

$$
\begin{aligned}
& \mathrm{BU}_{2}^{\left(\mathrm{E}_{8}, \mathrm{SU}(2)\right)}: \\
& \begin{array}{c|ccccccccc|cc|cc}
S_{i} \cdot D_{j}^{2} & D_{0}^{\mathrm{E} 8} & D_{1}^{\mathrm{E} 8} & D_{2}^{\mathrm{E} 8} & D_{3}^{\mathrm{E} 8} & D_{4}^{\mathrm{E}_{8}} & D_{5}^{\mathrm{E} 8} & D_{6}^{\mathrm{E}_{8}} & D_{7}^{\mathrm{E} 8} & D_{8}^{\mathrm{E} 8} & D_{0}^{\mathrm{SU}(2)} & D_{1}^{\mathrm{SU}(2)} & S_{1} & S_{2} \\
\hline S_{1} & 0 & 0 & 0 & 0 & 0 & 0 & 0 & 0 & 0 & 0 & 0 & 8 & 0 \\
S_{2} & 0 & -1 & -2 & -2 & -2 & -2 & -1 & 0 & -2 & 0 & 1 & -2 & 3 \\
\hline n\left(F_{j}\right) & 0 & -1 & -2 & -2 & -2 & -2 & -1 & 0 & -2 & 0 & 2 & - & -
\end{array} .
\end{aligned}
$$

Note that the numbers $n\left(F_{J}\right)$ is the same as for $\mathrm{BU}_{1}$ in (D.32), hence it has the same flavor symmetry and $M$ :

$$
G_{F}=\mathrm{SU}(6) \times \mathrm{U}(1), g=0, M=6, k=\frac{3}{2}, a=5 .
$$

Thus the only difference is the value of $a$. Geometrically, $\mathrm{BU}_{2}$ can be constructed from $\mathrm{BU}_{1}$ by flopping curves from $S_{1}$ into $S_{2}$. Field theoretically, they represent distinct gauge

\footnotetext{
${ }^{14}$ The difference between the two models is simply the placement of the resolution step $\left\{\delta_{1}, u_{3}, \delta_{2}\right\}$.
} 
theory phases for the same strongly coupled SCFT. We will systematically study how to characterize all the gauge theory descriptions and associated geometries, that give rise to the same SCFTs in [31]. Again we can determine the codimension-two fiber explicitly by considering the irreducible curve components:

\begin{tabular}{|c|c|c|c|c|c|c|}
\hline $\mathrm{E}_{8}$ Root & Irreducible Components & $C \cdot C$ & $S_{1}$ & $S_{2}$ & $D_{0}^{\mathrm{SU}(2)}$ & $D_{1}^{\mathrm{SU}(2)}$ \\
\hline$\alpha_{0}^{\mathrm{E}_{8}}$ & $D_{0}^{\mathrm{E}_{8}} \cdot D_{0}^{\mathrm{SU}(2)}$ & -2 & & & - & \\
\hline$\alpha_{1}^{\mathrm{E}_{8}}$ & $D_{1}^{\mathrm{E}_{8}} \cdot D_{0}^{\mathrm{SU}(2)}$ & -1 & & & - & \\
& $D_{1}^{\mathrm{E}_{8}} \cdot S_{1}$ & -2 & - & & & \\
& $D_{1}^{\mathrm{E}_{8}} \cdot S_{2}$ & -1 & & - & & \\
\hline$\alpha_{2}^{\mathrm{E}_{8}}$ & $D_{2}^{\mathrm{E}_{8}} \cdot S_{2}$ & -2 & & - & & \\
\hline$\alpha_{3}^{\mathrm{E}_{8}}$ & $D_{3}^{\mathrm{E}_{8}} \cdot S_{2}$ & -2 & & - & & \\
\hline$\alpha_{4}^{\mathrm{E}_{8}}$ & $D_{4}^{\mathrm{E}_{8}} \cdot S_{2}$ & -2 & & - & & \\
\hline$\alpha_{5}^{\mathrm{E} 8}$ & $D_{5}^{\mathrm{E}_{8}} \cdot S_{2}$ & -2 & & - & & \\
\hline$\alpha_{6}^{\mathrm{E}_{8}}$ & $D_{6}^{\mathrm{E}_{8}} \cdot S_{2}$ & -1 & & - & & \\
\hline$\alpha_{7}^{\mathrm{E}_{8}}$ & $D_{6}^{\mathrm{E}_{8}} \cdot D_{1}^{\mathrm{SU}(2)}$ & -1 & & & & - \\
\hline$\alpha_{8}^{\mathrm{E}_{8}}$ & $D_{7}^{\mathrm{E}_{8}} \cdot D_{1}^{\mathrm{SU}(2)}$ & -2 & & & & - \\
\hline & $D_{8}^{\mathrm{E}_{8}} \cdot S_{2}$ & -2 & & - & & \\
\hline
\end{tabular}

The resulting codimension two fiber is shown in figure 7 .

We close with another example blow-up where the 5d SCFT is different from the above models: consider

$$
\begin{aligned}
& \mathrm{BU}_{3}^{\left(\mathrm{E}_{8}, \mathrm{SU}(2)\right)}= \\
& \left\{\left\{x, y, U, u_{1}\right\},\left\{x, y, u_{1}, u_{2}\right\},\left\{x, y, V, v_{1}\right\},\left\{y, u_{2}, u_{3}\right\},\left\{V, u_{3}, \delta_{1}\right\},\left\{y, u_{1}, u_{3}, u_{4}\right\}\right. \\
& \quad\left\{y, u_{1}, u_{5}\right\},\left\{u_{1}, u_{3}, u_{6}\right\},\left\{u_{1}, u_{4}, u_{7}\right\},\left\{u_{1}, u_{5}, u_{8}\right\},\left\{u_{2}, u_{3}, u_{9}\right\},\left\{u_{3}, u_{4}, u_{10}\right\}, \\
& \left\{u_{4}, u_{6}, u_{11}\right\},\left\{u_{3}, u_{6}, u_{12}\right\},\left\{u_{6}, u_{10}, u_{13}\right\},\left\{u_{10}, u_{12}, u_{14}\right\},\left\{u_{3}, u_{12}, u_{15}\right\} \\
& \left.\quad\left\{\delta_{1}, u_{3}, \delta_{2}\right\}\right\}
\end{aligned}
$$

The reduced intersection matrix is

$$
\begin{aligned}
& \mathrm{BU}_{3}^{\left(\mathrm{E}_{8}, \mathrm{SU}(2)\right)}: \\
& \begin{array}{c|ccccccccc|cc|cc}
S_{i} \cdot D_{j}^{2} & D_{0}^{\mathrm{E} 8} & D_{1}^{\mathrm{E}_{8}} & D_{2}^{\mathrm{E}_{8}} & D_{3}^{\mathrm{E} 8} & D_{4}^{\mathrm{E}_{8}} & D_{5}^{\mathrm{E}_{8}} & D_{6}^{\mathrm{E}_{8}} & D_{7}^{\mathrm{E}_{8}} & D_{8}^{\mathrm{E}_{8}} & D_{0}^{\mathrm{SU}(2)} & D_{1}^{\mathrm{SU}(2)} & S_{1} & S_{2} \\
\hline S_{1} & 0 & -1 & -2 & -2 & -2 & -1 & 0 & 0 & 0 & 0 & 0 & 4 & -4 \\
S_{2} & 0 & 0 & 0 & 0 & 0 & -1 & -2 & -1 & -2 & 0 & 0 & 2 & 6 \\
\hline n\left(F_{j}\right) & 0 & -1 & -2 & -2 & -2 & -2 & -2 & -1 & -2 & 0 & 0 & - & -
\end{array}
\end{aligned}
$$

and thus we can read off

$$
G_{F, n a}=\mathrm{SO}(12), g=0, M=7, k=2, a=2 .
$$


The irreducible fiber components are likewise obtained from the splitting of the roots as follows:

\begin{tabular}{|c|c|c|c|c|c|c|}
\hline $\mathrm{E}_{8}$ Root & Irreducible Components & $C \cdot C$ & $S_{1}$ & $S_{2}$ & $D_{0}^{\mathrm{SU}(2)}$ & $D_{1}^{\mathrm{SU}(2)}$ \\
\hline$\alpha_{0}^{\mathrm{E}_{8}}$ & $D_{0}^{\mathrm{E}_{8}} \cdot D_{0}^{\mathrm{SU}(2)}$ & -2 & & & - & \\
\hline$\alpha_{1}^{\mathrm{E}_{8}}$ & $D_{1}^{\mathrm{E}_{8}} \cdot D_{0}^{\mathrm{SU}(2)}$ & -1 & & & - & \\
& $D_{1}^{\mathrm{E}_{8}} \cdot S_{1}$ & -1 & - & & & \\
\hline$\alpha_{2}^{\mathrm{E}_{8}}$ & $D_{2}^{\mathrm{E}_{8}} \cdot S_{1}$ & -2 & - & & & \\
\hline$\alpha_{3}^{\mathrm{E}_{8}}$ & $D_{3}^{\mathrm{E}_{8}} \cdot S_{1}$ & -2 & - & & & \\
\hline$\alpha_{4}^{\mathrm{E}_{8}}$ & $D_{4}^{\mathrm{E}_{8}} \cdot S_{1}$ & -2 & - & & & \\
\hline$\alpha_{5}^{\mathrm{E}_{8}}$ & $D_{5}^{\mathrm{E}_{8}} \cdot S_{1}$ & -1 & - & & & \\
\hline$\alpha_{6}^{\mathrm{E}_{8}}$ & $D_{5}^{\mathrm{E}_{8}} \cdot S_{2}$ & -1 & & - & & \\
\hline$\alpha_{7}^{\mathrm{E}_{8}}$ & $D_{6}^{\mathrm{E}_{8}} \cdot D_{1}^{\mathrm{SU}(2)}$ & -2 & & & & - \\
\hline$\alpha_{8}^{\mathrm{E}_{8}}$ & $D_{7}^{\mathrm{E}_{8}} \cdot S_{2}$ & -1 & & - & & \\
\hline
\end{tabular}

Again the codimension two fiber is shown in figure 7 .

For the abelian part of the flavor symmetry, we compute the rank of the following intersection matrix, which turns out to be nine:

\begin{tabular}{c|ccccccccc|cc} 
& $D_{0}^{\mathrm{E}_{8}}$ & $D_{1}^{\mathrm{E}_{8}}$ & $D_{2}^{\mathrm{E}_{8}}$ & $D_{3}^{\mathrm{E}_{8}}$ & $D_{4}^{\mathrm{E}_{8}}$ & $D_{5}^{\mathrm{E}_{8}}$ & $D_{6}^{\mathrm{E}_{8}}$ & $D_{7}^{\mathrm{E}_{8}}$ & $D_{8}^{\mathrm{E}_{8}}$ & $D_{0}^{\mathrm{SU}(2)}$ & $D_{1}^{\mathrm{SU}(2)}$ \\
\hline$D_{1}^{\mathrm{E}_{8}} \cdot S_{1}$ & 0 & -1 & 1 & 0 & 0 & 0 & 0 & 0 & 0 & 1 & 0 \\
$D_{2}^{\mathrm{E}_{8}} \cdot S_{1}$ & 0 & 1 & -2 & 1 & 0 & 0 & 0 & 0 & 0 & 0 & 0 \\
$D_{3}^{\mathrm{E}_{8}} \cdot S_{1}$ & 0 & 0 & 1 & -2 & 1 & 0 & 0 & 0 & 0 & 0 & 0 \\
$D_{4}^{\mathrm{E} 8} \cdot S_{1}$ & 0 & 0 & 0 & 1 & -2 & 1 & 0 & 0 & 0 & 0 & 0 \\
$D_{5}^{\mathrm{E}_{8}} \cdot S_{1}$ & 0 & 0 & 0 & 0 & 1 & -1 & 0 & 0 & 0 & 0 & 0 \\
$D_{5}^{\mathrm{E}_{8}} \cdot S_{2}$ & 0 & 0 & 0 & 0 & 0 & -1 & 1 & 0 & 1 & 0 & 0 \\
$D_{6}^{\mathrm{E}} \cdot S_{2}$ & 0 & 0 & 0 & 0 & 0 & 1 & -2 & 1 & 0 & 0 & 0 \\
$D_{7}^{\mathrm{E}} \cdot S_{2}$ & 0 & 0 & 0 & 0 & 0 & 0 & 1 & -1 & 0 & 0 & 1 \\
$D_{8}^{\mathrm{E}_{8}} \cdot S_{2}$ & 0 & 0 & 0 & 0 & 0 & 1 & 0 & 0 & -2 & 0 & 0 \\
$D_{0}^{\mathrm{SU}(2)} \cdot S_{1}$ & 0 & 1 & 0 & 0 & 0 & 0 & 0 & 0 & 0 & 0 & 2 \\
$D_{1}^{\mathrm{SU}(2)} \cdot S_{1}$ & 0 & 0 & 0 & 0 & 0 & 0 & 0 & 0 & 0 & 2 & 0 \\
$D_{1}^{\mathrm{SU}(2)} \cdot S_{2}$ & 0 & 0 & 0 & 0 & 0 & 0 & 0 & 1 & 0 & 0 & 0
\end{tabular}

Substract nine by the rank of $\mathrm{SU}(3)_{G}$, we get the total rank of flavor symmetry $M=7$. Hence the total flavor symmetry is

$$
G_{\mathrm{F}}=\mathrm{SO}(12) \times \mathrm{U}(1) .
$$

This concludes our examples of concrete blow-ups of the rank two E-string. The process is pretty clear from what we have described and many more models can be obtained in this way, by resolving the singularity including the non-minimal one in codimension two. We will next pass to the $D_{10}-I_{1}$ starting point and illustrate the blow-ups in this case as well. 

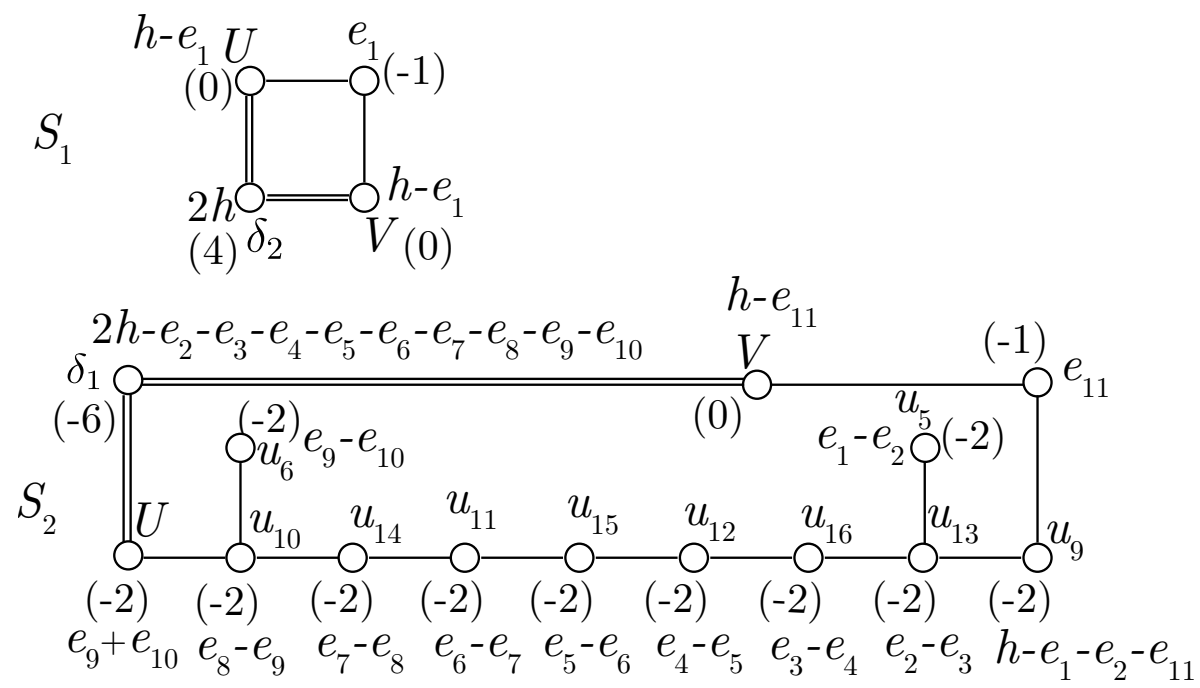

Figure 23. The configuration of curves on $S_{1}$ and $S_{2}$ in the geometry that has $M=11$ for the $D_{10}-I_{1}$ collision. The number in the bracket denotes the self-intersection number of the curve. The letter denotes an intersection curve with the corresponding divisor.

The main observation from this section is that there are multiple resolutions, that will correspond to different gauge theory realizations of the same SCFT. The key to characterizing the distinct 5d SCFTs will be removing this redundant information and extracting the relevant fiber information that uniquely fixes the SCFT, which will be explained in section 4 .

\section{D.3 Blow-ups for the $\left(D_{10}, I_{1}\right)$ conformal matter}

\section{D.3.1 Geometry for the marginal theory}

To get the marginal geometry with $M=11$, we blow up the base locus $U=V=0$ first: $\left(U, V ; \delta_{1}\right)$. The starting point Tate model is then:

$$
y^{2}+b_{1} U x y+b_{3} U^{5} \delta_{1}^{2}=x^{3}+b_{2} U V x^{2}+b_{4} U^{5} \delta_{1} x+b_{6} U^{10} \delta_{1}^{4}
$$

We can choose the following blow-up chain:

$$
\begin{aligned}
& \mathrm{BU}_{M=11}^{\left(D_{10}, I_{1}\right)}= \\
& \left\{\left\{x, y, \delta_{1}, \delta_{2}\right\}\right\},\left\{\left\{x, y, U, u_{1}\right\},\left\{x, y, u_{1}, u_{2}\right\},\left\{x, y, u_{2}, u_{3}\right\},\left\{x, y, u_{3}, u_{4}\right\},\left\{x, y, u_{4}, u_{5}\right\},\right. \\
& \left\{y, u_{1}, u_{6}\right\},\left\{y, u_{2}, u_{7}\right\},\left\{y, u_{3}, u_{8}\right\},\left\{y, u_{4}, u_{9}\right\},\left\{u_{1}, u_{6}, u_{10}\right\},\left\{u_{2}, u_{7}, u_{11}\right\}, \\
& \left.\left\{u_{3}, u_{8}, u_{12}\right\},\left\{u_{4}, u_{9}, u_{13}\right\},\left\{u_{2}, u_{6}, u_{14}\right\},\left\{u_{3}, u_{7}, u_{15}\right\},\left\{u_{4}, u_{8}, u_{16}\right\}\right\}
\end{aligned}
$$

The ordering of the simple roots for $\mathrm{SO}(20)$ is as in [46] with the sections

$$
\begin{aligned}
\left(D_{0}, D_{1}, \cdots,\right. & \left.D_{10}\left|D_{0}^{I_{1}}\right| S_{1}, S_{2}\right) \\
& \equiv\left(U, u_{6}, u_{10}, u_{14}, u_{11}, u_{15}, u_{12}, u_{16}, u_{13}, u_{9}, u_{5}|V| \delta_{1}, \delta_{2}\right) .
\end{aligned}
$$

Here $D_{i}$ is associated to the $i$ th simple root of $\mathrm{SO}(20)$. 
The configuration of is shown in figure 23 and

$$
\begin{aligned}
& \mathrm{BU}_{M=11}^{\left(D_{10}, I_{1}\right)}= \\
& \begin{array}{c|ccccccccccc|c|cc}
S_{i} \cdot D_{j}^{2} & D_{0} & D_{1} & D_{2} & D_{3} & D_{4} & D_{5} & D_{6} & D_{7} & D_{8} & D_{9} & D_{10} & D_{0}^{I_{1}} & S_{1} & S_{2} \\
\hline S_{1} & 0 & 0 & 0 & 0 & 0 & 0 & 0 & 0 & 0 & 0 & 0 & 0 & 8 & 4 \\
S_{2} & -2 & -2 & -2 & -2 & -2 & -2 & -2 & -2 & -2 & -2 & -2 & 0 & -6 & -2 \\
\hline n\left(F_{j}\right) & -2 & -2 & -2 & -2 & -2 & -2 & -2 & -2 & -2 & -2 & -2 & 0 & - & -
\end{array} .
\end{aligned}
$$

Note that the curve $D_{0} \cdot S_{2}$ is a reducible $(-2)$-curve on $S_{2}$. Nonetheless, the curve $D_{0} \cdot\left(S_{1}+S_{2}\right)$ is still a rational $(-2)$-curve, since we can compute from figure 23

$$
D_{0}^{2} \cdot\left(S_{1}+S_{2}\right)=-2, D_{0} \cdot\left(S_{1}+S_{2}\right)^{2}=0 .
$$

\section{D.3.2 Example resolution for $\left(D_{10}, I_{1}\right)$}

We consider an example resolution of a descendant theory:

$$
\begin{gathered}
\mathrm{BU}_{1}^{\left(D_{10}, I_{1}\right)}= \\
\left\{\left\{x, y, U, u_{1}\right\},\left\{x, y, u_{1}, u_{2}\right\},\left\{x, y, u_{2}, u_{3}\right\},\left\{x, y, u_{3}, u_{4}\right\},\left\{x, y, u_{4}, u_{5}\right\},\left\{y, u_{1}, u_{6}\right\},\right. \\
\left\{y, u_{2}, u_{7}\right\},\left\{y, u_{3}, u_{8}\right\},\left\{y, u_{4}, u_{9}\right\},\left\{u_{1}, u_{6}, u_{10}\right\},\left\{u_{2}, u_{7}, u_{11}\right\},\left\{u_{3}, u_{8}, u_{12}\right\}, \\
\left.\left\{u_{4}, u_{9}, u_{13}\right\},\left\{u_{2}, u_{6}, u_{14}\right\},\left\{u_{3}, u_{7}, u_{15}\right\},\left\{u_{4}, u_{8}, u_{16}\right\},\left\{V, u_{7}, \delta_{1}\right\},\left\{V, u_{8}, \delta_{2}\right\}\right\} .
\end{gathered}
$$

The reduced intersection matrix is then:

$$
\begin{array}{r|ccccccccccc|c|cc}
S_{i} \cdot D_{j}^{2} & D_{0} & D_{1} & D_{2} & D_{3} & D_{4} & D_{5} & D_{6} & D_{7} & D_{8} & D_{9} & D_{10} & D_{0}^{I_{1}} & S_{1} & S_{2} \\
\hline S_{1} & 0 & 0 & 0 & 0 & -1 & -2 & -1 & 0 & 0 & 0 & 0 & 4 & 6 & -2 \\
S_{2} & 0 & 0 & 0 & 0 & 0 & 0 & -1 & -2 & -1 & -2 & 0 & 2 & 0 & 6 \\
\hline n\left(F_{j}\right) & 0 & 0 & 0 & 0 & -1 & -2 & -2 & -2 & -1 & -2 & 0 & 6 & - & -
\end{array}
$$

We can read off

$$
G_{F, n a}=\mathrm{SU}(4) \times \mathrm{SU}(2), g=0, M=5, k=1, a=2 .
$$

The corresponding codimension two fiber is shown in figure 8 .

We can also compute the total rank of flavor symmetry group $G_{\mathrm{F}}$ as the rank of the following intersection matrix, which turns out to be seven:

\begin{tabular}{c|ccccccccccc} 
& $D_{0}$ & $D_{1}$ & $D_{2}$ & $D_{3}$ & $D_{4}$ & $D_{5}$ & $D_{6}$ & $D_{7}$ & $D_{8}$ & $D_{9}$ & $D_{10}$ \\
\hline$D_{3} \cdot S_{1}$ & 0 & 0 & 0 & 0 & 1 & 0 & 0 & 0 & 0 & 0 & 0 \\
$D_{4} \cdot S_{1}$ & 0 & 0 & 0 & 1 & -1 & 1 & 0 & 0 & 0 & 0 & 0 \\
$D_{5} \cdot S_{1}$ & 0 & 0 & 0 & 0 & 1 & -2 & 1 & 0 & 0 & 0 & 0 \\
$D_{6} \cdot S_{1}$ & 0 & 0 & 0 & 0 & 0 & 1 & -1 & 0 & 0 & 0 & 0 \\
$D_{6} \cdot S_{2}$ & 0 & 0 & 0 & 0 & 0 & 0 & -1 & 1 & 0 & 0 & 0 \\
$D_{7} \cdot S_{2}$ & 0 & 0 & 0 & 0 & 0 & 0 & 1 & -2 & 1 & 0 & 0 \\
$D_{8} \cdot S_{2}$ & 0 & 0 & 0 & 0 & 0 & 0 & 0 & 1 & -1 & 1 & 0 \\
$D_{9} \cdot S_{2}$ & 0 & 0 & 0 & 0 & 0 & 0 & 0 & 0 & 1 & -2 & 0
\end{tabular}

Substract seven by the rank of $\mathrm{SU}(3)_{G}$, we confirmed $M=5$ and the total flavor symmetry is

$$
G_{\mathrm{F}}=\mathrm{SU}(4) \times \mathrm{SU}(2) \times \mathrm{U}(1) .
$$




\section{D.4 Resolution of $\left(\mathrm{E}_{8}, \mathrm{SU}(3)\right)$ and $\left(\mathrm{E}_{8}, G_{2}\right)$}

We give an example of a non-flat resolution of the collision $I I^{*}$ and $I_{3}$ Kodaira fibers associated to the $\left(\mathrm{E}_{8}, \mathrm{SU}(3)\right)$ conformal matter. The 5d SCFTs obtained from this have rank four. The sequence of blow-ups that we will consider is

$$
\begin{aligned}
& \mathrm{BU}^{\left(\mathrm{E}_{8}, \mathrm{SU}(3)\right)}= \\
& \qquad\left\{x, y, u, u_{1}\right\},\left\{x, y, v, v_{1}\right\},\left\{x, y, u_{1}, u_{2}\right\},\left\{y, v_{1}, v_{2}\right\}, \\
& \quad\left\{u_{1}, v_{2}, \delta_{1}\right\},\left\{u_{2}, v_{2}, \delta_{2}\right\},\left\{y, u_{2}, u_{3}\right\},\left\{u_{3}, \delta_{1}, \delta_{3}\right\},\left\{y, u_{1}, u_{3}, u_{4}\right\},\left\{y, u_{1}, u_{5}\right\}, \\
& \quad\left\{u_{1}, u_{3}, u_{6}\right\},\left\{u_{1}, u_{4}, u_{7}\right\},\left\{u_{1}, u_{5}, u_{8}\right\},\left\{u_{2}, u_{3}, u_{9}\right\},\left\{u_{3}, \delta_{3}, \delta_{4}\right\},\left\{u_{3}, u_{4}, u_{10}\right\}, \\
& \left.\quad\left\{u_{4}, u_{6}, u_{11}\right\},\left\{u_{3}, u_{6}, u_{12}\right\},\left\{u_{6}, u_{10}, u_{13}\right\},\left\{u_{10}, u_{12}, u_{14}\right\},\left\{u_{3}, u_{12}, u_{15}\right\}\right\}
\end{aligned}
$$

The ordering of the $\mathrm{E}_{8}$ Cartan divisors is in the rank one and two examples. The $\mathrm{SU}(3)$ affine roots are identified with $V, v_{1}, v_{2}$, and the non-flat surface components $S_{i}$ are given by $\delta_{i}=0$. The reduced triple intersection matrix of the Cartan divisors $D_{i}^{\mathfrak{g}}$ of $\mathrm{E}_{8}$ and $\mathrm{SU}(3)$, respectively, with the four non-flat fiber components $S_{i}$ are

$$
\mathrm{BU}^{\left(\mathrm{E}_{8}, \mathrm{SU}(3)\right)} \text { : }
$$

\begin{tabular}{c|ccccccccc|ccc|cccc}
$S_{i} \cdot D_{j}^{2}$ & $D_{0}$ & $D_{1}$ & $D_{2}$ & $D_{3}$ & $D_{4}$ & $D_{5}$ & $D_{6}$ & $D_{7}$ & $D_{8}$ & $D_{0}^{I_{3}}$ & $D_{1}^{I_{3}}$ & $D_{2}^{I_{3}}$ & $S_{1}$ & $S_{2}$ & $S_{3}$ & $S_{4}$ \\
\hline$S_{1}$ & 0 & 0 & 0 & 0 & 0 & 0 & 0 & 0 & 0 & 0 & -1 & -1 & 6 & -2 & -2 & 0 \\
$S_{2}$ & 0 & 0 & 0 & 0 & 0 & 0 & 0 & -1 & 0 & 0 & -1 & -1 & -2 & 4 & -4 & -2 \\
$S_{3}$ & 0 & -1 & -2 & -1 & 0 & 0 & 0 & 0 & 0 & 0 & 0 & 0 & 0 & 0 & 6 & -2 \\
$S_{4}$ & 0 & 0 & 0 & -1 & -2 & -2 & -2 & -1 & -2 & 0 & 0 & 0 & 0 & 0 & 0 & 4 \\
\hline$n\left(F_{j}\right)$ & 0 & -1 & -2 & -2 & -2 & -2 & -2 & -2 & -2 & 0 & -2 & -2 & - & - & - & -
\end{tabular}

The wrapped components of the fiber and codimension two fiber is shown in figure 9 . The strongly coupled flavor symmetry for the SCFT from this point of view is

$$
G_{\mathrm{F}} \supset \mathrm{E}_{7} \times \mathrm{SU}(3) .
$$

With some minor changes we can generalize this to $\left(\mathrm{E}_{8}, G_{2}\right)$, i.e., the collision of $I I^{*}$ with $I_{1}^{n s}$ (non-split $I_{1}^{*}$ ). The vanishing orders change to

$$
\operatorname{ord}_{v=0}\left(b_{i}\right)=(0,1,1,2,3) \text {. }
$$

The same resolution sequence can be applied, except in order to obtain the $G_{2}$ we need to perform another small resolution

$$
\mathrm{BU}^{\left(\mathrm{E}_{8}, G_{2}\right)}=\mathrm{BU}^{\left(\mathrm{E}_{8}, \mathrm{SU}(3)\right)} \cup\left\{v_{1}, v_{2}, v_{3}\right\} .
$$

The roots of the affine $G_{2}$ are identified with $\alpha_{0} \leftrightarrow V, \alpha_{1} \leftrightarrow v_{3}, \alpha_{2} \leftrightarrow v_{2}$, so that the intersection matrix for the $G_{2}$ part of the codimension one singular fibers is

$$
-C_{i j}^{\widehat{\mathrm{g}}_{2}}=\left(\begin{array}{ccc}
-2 & 1 & 0 \\
1 & -2 & 3 \\
0 & 3 & -6
\end{array}\right) .
$$


The reduced triple intersection matrix is now

$\mathrm{BU}^{\left(\mathrm{E}_{8}, G_{2}\right)}:$
\begin{tabular}{c|ccccccccc|ccc|cccc}
$S_{i} \cdot D_{j}^{2}$ & $D_{0}$ & $D_{1}$ & $D_{2}$ & $D_{3}$ & $D_{4}$ & $D_{5}$ & $D_{6}$ & $D_{7}$ & $D_{8}$ & $D_{0}^{G_{2}}$ & $D_{1}^{G_{2}}$ & $D_{2}^{G_{2}}$ & $S_{1}$ & $S_{2}$ & $S_{3}$ & $S_{4}$ \\
\hline$S_{1}$ & 0 & 0 & 0 & 0 & 0 & 0 & 0 & 0 & 0 & 0 & -1 & -2 & 6 & -2 & -2 & 0 \\
$S_{2}$ & 0 & 0 & 0 & 0 & 0 & 0 & 0 & -1 & 0 & 0 & -1 & -4 & -2 & 4 & -4 & -2 \\
$S_{3}$ & 0 & -1 & -2 & -1 & 0 & 0 & 0 & 0 & 0 & 0 & 0 & 0 & 0 & 0 & 6 & -2 \\
$S_{4}$ & 0 & 0 & 0 & -1 & -2 & -2 & -2 & -1 & -2 & 0 & 0 & 0 & 0 & 0 & 0 & 4 \\
\hline$n\left(F_{j}\right)$ & 0 & -1 & -2 & -2 & -2 & -2 & -2 & -2 & -2 & 0 & -2 & -6 & - & - & - & -
\end{tabular}

So here the flavor symmetry at the strongly coupled point is

$$
G_{\mathrm{F}}=\mathrm{E}_{7} \times G_{2} .
$$

The fiber is depicted in figure 9.

\section{D.5 Higher rank marginal theories}

The $\left(\mathrm{E}_{8}, \mathrm{SU}(2 k)\right)$ conformal matter theory is a $6 \mathrm{~d}(1,0) \mathrm{SCFT}$ with rank $2 k^{2}-k+1$. The tensor branch configuration is

$$
\left[\mathrm{E}_{8}\right]-1-2-\stackrel{\mathfrak{s u}_{2}}{2}-\stackrel{\mathfrak{s u}}{2}^{\mathfrak{s u}_{3}} \cdots-\frac{\mathfrak{s u}_{2 k-1}}{2}-[\mathrm{SU}(2 k)] .
$$

Starting with the Weierstrass model:

$$
y^{2}+b_{1} U x y+b_{3} U^{3} V^{k} y=x^{3}+b_{2} U^{2} V x^{2}+b_{4} U^{4} V^{k} x+b_{6} U^{5} V^{2 k},
$$

we first blow up the base locus $\left(U, V ; \delta_{1}\right)$, and then use the following resolution sequence:

$$
\begin{aligned}
& \mathrm{BU}_{\text {marginal }}^{\left(\mathrm{E}_{8}, \mathrm{SU}(2 k)\right)}= \\
& \left\{\left\{x, y, U, u_{1}\right\},\left\{x, y, u_{1}, u_{2}\right\},\left\{y, u_{2}, u_{3}\right\},\left\{y, u_{1}, u_{3}, u_{4}\right\},\left\{y, u_{1}, u_{5}\right\},\left\{u_{1}, u_{3}, u_{6}\right\},\right. \\
& \quad\left\{u_{1}, u_{4}, u_{7}\right\},\left\{u_{1}, u_{5}, u_{8}\right\},\left\{u_{2}, u_{3}, u_{9}\right\},\left\{u_{3}, u_{4}, u_{10}\right\},\left\{u_{4}, u_{6}, u_{11}\right\}, \\
& \left\{u_{3}, u_{6}, u_{12}\right\},\left\{u_{6}, u_{10}, u_{13}\right\},\left\{u_{10}, u_{12}, u_{14}\right\},\left\{u_{3}, u_{12}, u_{15}\right\}, \\
& \left\{x, y, V, v_{1}\right\},\left\{x, y, v_{i}, v_{i+1}\right\}(i=1, \ldots, k-1), \\
& \left\{y, v_{i}, v_{i+k}\right\}(i=1, \ldots, k-1),\left\{u_{3}, \delta_{i}, \delta_{i+1}\right\}(i=1, \ldots, 2 k-1), \\
& {\left[\left\{x, y, \delta_{2 i-1}, \delta_{2 i k-i^{2}+i+1}\right\},\right.} \\
& \left\{x, y, \delta_{2 i k-i^{2}+i+j+1}, \delta_{2 i k-i^{2}+i+j+2}\right\}(j=0, \cdots, k-i-2), \\
& \left.\quad\left\{y, \delta_{2 i k-i^{2}+i+j+1}, \delta_{2 i k-i^{2}+k+j+1}\right\}(j=0, \ldots, k-i-1)\right](i=1, \cdots, k-1), \\
& {\left[\left\{x, y, \delta_{2 i}, \delta_{k^{2}+2 k i-k-i^{2}+2}\right\},\right.} \\
& \left\{x, y, \delta_{k^{2}+2 k i-k-i^{2}+j+2}, \delta_{k^{2}+2 k i-k-i^{2}+j+3}\right\}(j=0, \cdots, k-i-2), \\
& \left.\left\{x, y, \delta_{k^{2}+2 k i-k-i^{2}+j+2}, \delta_{k^{2}+2 k i-i-i^{2}+j+2}\right\}(j=0, \cdots, k-i-2)\right] \\
& \quad(i=1, \cdots, k-1)\}
\end{aligned}
$$


The $\left(\mathrm{E}_{8}, \mathrm{SU}(2 k+1)\right)$ conformal matter theory is a $6 \mathrm{~d}(1,0) \mathrm{SCFT}$ with rank $2 k^{2}+k+1$. The tensor branch configuration is

$$
\left[\mathrm{E}_{8}\right]-1-2-\stackrel{\mathfrak{s u}_{2}}{2}-\stackrel{\mathfrak{s u}}{2}^{\mathfrak{s u}_{3}} \cdots-\stackrel{\mathfrak{s u}}{2 k}^{\mathfrak{s u}_{2}}-[\mathrm{SU}(2 k+1)] .
$$

Starting with the Weierstrass model:

$$
y^{2}+b_{1} U x y+b_{3} U^{3} V^{k} y=x^{3}+b_{2} U^{2} V x^{2}+b_{4} U^{4} V^{k+1} x+b_{6} U^{5} V^{2 k+1},
$$

we first blow up the base locus $\left(U, V ; \delta_{1}\right)$, and then use the following resolution sequence:

$$
\begin{aligned}
& \mathrm{BU}_{\text {marginal }}^{\left(\mathrm{E}_{8}, \mathrm{SU}(2 k+1)\right)}= \\
& \left\{\left\{x, y, U, u_{1}\right\},\left\{x, y, u_{1}, u_{2}\right\},\left\{y, u_{2}, u_{3}\right\},\left\{y, u_{1}, u_{3}, u_{4}\right\},\left\{y, u_{1}, u_{5}\right\},\left\{u_{1}, u_{3}, u_{6}\right\},\right. \\
& \quad\left\{u_{1}, u_{4}, u_{7}\right\},\left\{u_{1}, u_{5}, u_{8}\right\},\left\{u_{2}, u_{3}, u_{9}\right\},\left\{u_{3}, u_{4}, u_{10}\right\},\left\{u_{4}, u_{6}, u_{11}\right\},\left\{u_{3}, u_{6}, u_{12}\right\}, \\
& \quad\left\{u_{6}, u_{10}, u_{13}\right\},\left\{u_{10}, u_{12}, u_{14}\right\},\left\{u_{3}, u_{12}, u_{15}\right\}, \\
& \quad\left\{x, y, V, v_{1}\right\},\left\{x, y, v_{i}, v_{i+1}\right\}(i=1, \ldots, k-1), \\
& \left\{y, v_{i}, v_{i+k}\right\}(i=1, \ldots, k),\left\{u_{3}, \delta_{i}, \delta_{i+1}\right\}(i=1, \ldots, 2 k), \\
& {\left[\left\{x, y, \delta_{2 i-1}, \delta_{2 i k-i^{2}+2 i+1}\right\},\right.} \\
& \quad\left\{x, y, \delta_{2 i k-i^{2}+2 i+j+1}, \delta_{2 i k-i^{2}+2 i+j+2}\right\}(j=0, \cdots, k-i-2), \\
& \left.\quad\left\{y, \delta_{2 i k-i^{2}+2 i+j+1}, \delta_{2 i k-i^{2}+i+j+k+2}\right\}(j=0, \ldots, k-i-1)\right](i=1, \cdots, k-1), \\
& {\left[\left\{x, y, \delta_{2 i}, \delta_{k^{2}+2 k i-i^{2}+i+2}\right\},\right.} \\
& \quad\left\{x, y, \delta_{k^{2}+2 k i-i^{2}+i+j+2}, \delta_{k^{2}+2 k i-i^{2}+i+j+3}\right\}(j=0, \cdots, k-i-2), \\
& \left.\quad\left\{x, y, \delta_{k^{2}+2 k i-i^{2}+i+j+2}, \delta_{k^{2}+2 k i-i^{2}+j+k+2}\right\}(j=0, \cdots, k-i-2)\right] \\
& \quad(i=1, \cdots, k-1)\}
\end{aligned}
$$

We also present the marginal geometry of $\left(\mathrm{E}_{6}, \mathrm{E}_{6}\right)$ conformal matter theory mentioned in [8], with the following the tensor branch configuration is

$$
\left[\mathrm{E}_{6}\right]-1-\stackrel{\mathfrak{s u}_{3}}{3}-1-\left[\mathrm{E}_{6}\right]
$$

Starting with the Weierstrass model:

$$
y^{2}+b_{1} U V x y+b_{3} U^{2} V^{2} y=x^{3}+b_{2} U^{2} V^{2} x^{2}+b_{4} U^{3} V^{3} x+b_{6} U^{5} V^{5},
$$

we first blow up the base locus $\left(U, V ; \delta_{1}\right)$, and then use the following resolution sequence:

$$
\begin{aligned}
& \mathrm{BU}_{\text {marginal }}^{\left(\mathrm{E}_{6}, \mathrm{E}_{6}\right)}= \\
& \left\{\left\{x, y, U, u_{1}\right\},\left\{x, y, u_{1}, u_{2}\right\},\left\{y, u_{1}, u_{2}, u_{3}\right\},\left\{y, u_{1}, u_{4}\right\},\left\{y, u_{2}, u_{5}\right\},\left\{u_{3}, u_{4}, u_{6}\right\},\right. \\
& \quad\left\{y, u_{3}, u_{7}\right\},\left\{u_{1}, u_{4}, u_{8}\right\},\left\{x, y, V, v_{1}\right\},\left\{x, y, v_{1}, v_{2}\right\},\left\{y, v_{1}, v_{2}, v_{3}\right\},\left\{y, v_{1}, v_{4}\right\}, \\
& \left\{y, v_{2}, v_{5}\right\},\left\{v_{3}, v_{4}, v_{6}\right\},\left\{y, v_{3}, v_{7}\right\},\left\{v_{1}, v_{4}, v_{8}\right\}, \\
& \left.\left\{u_{5}, \delta_{1}, \delta_{2}\right\},\left\{v_{5}, \delta_{1}, \delta_{3}\right\},\left\{x, y, \delta_{1}, \delta_{4}\right\},\left\{y, \delta_{4}, \delta_{5}\right\}\right\} .
\end{aligned}
$$


The $\left(\mathrm{E}_{7}, \mathrm{E}_{7}\right)$ conformal matter theory is a $6 \mathrm{~d}(1,0) \mathrm{SCFT}$ with rank 10 . The tensor branch configuration is

$$
\left[\mathrm{E}_{7}\right]-1-\stackrel{\mathfrak{s u}_{2}}{2}-\stackrel{\mathfrak{s o}_{7}}{3}-\stackrel{\mathfrak{s u}_{2}}{2}-1-\left[\mathrm{E}_{7}\right]
$$

Starting with the Weierstrass model:

$$
y^{2}+b_{1} U V x y+b_{3} U^{3} V^{3} y=x^{3}+b_{2} U^{2} V^{2} x^{2}+b_{4} U^{3} V^{3} x+b_{6} U^{5} V^{5},
$$

we first blow up the base locus $\left(U, V ; \delta_{1}\right)$, and then use the following resolution sequence:

$$
\begin{aligned}
& \mathrm{BU}_{\text {marginal }}^{\left(\mathrm{E}_{7}, \mathrm{E}_{7}\right)}= \\
& \left\{\left\{x, y, U, u_{1}\right\},\left\{x, y, u_{1}, u_{2}\right\},\left\{y, u_{1}, u_{3}\right\},\left\{y, u_{2}, u_{4}\right\},\left\{u_{2}, u_{3}, u_{5}\right\},\left\{u_{1}, u_{3}, u_{6}\right\},\right. \\
& \quad\left\{u_{2}, u_{4}, u_{7}\right\},\left\{u_{3}, u_{4}, u_{8}\right\},\left\{u_{4}, u_{5}, u_{9}\right\},\left\{u_{5}, u_{8}, u_{10}\right\},\left\{u_{3}, u_{5}, u_{11}\right\}, \\
& \quad\left\{x, y, V, v_{1}\right\},\left\{x, y, v_{1}, v_{2}\right\},\left\{y, v_{1}, v_{3}\right\},\left\{y, v_{2}, v_{4}\right\},\left\{v_{2}, v_{3}, v_{5}\right\},\left\{v_{1}, v_{3}, v_{6}\right\}, \\
& \\
& \quad\left\{v_{2}, v_{4}, v_{7}\right\},\left\{v_{3}, v_{4}, v_{8}\right\},\left\{v_{4}, v_{5}, v_{9}\right\},\left\{v_{5}, v_{8}, v_{10}\right\},\left\{v_{3}, v_{5}, v_{11}\right\}, \\
& \\
& \left\{u_{4}, \delta_{1}, \delta_{6}\right\},\left\{v_{4}, \delta_{1}, \delta_{4}\right\},\left\{u_{4}, \delta_{6}, \delta_{9}\right\},\left\{v_{4}, \delta_{4}, \delta_{8}\right\},\left\{x, y, \delta_{1}, \epsilon_{1}\right\}, \\
& \\
& \left.\left\{y, \epsilon_{1}, \delta_{2}\right\},\left\{\epsilon_{1}, \delta_{2}, \delta_{3}\right\},\left\{x, y, \delta_{4}, \delta_{5}\right\},\left\{x, y, \delta_{6}, \delta_{7}\right\}\right\} .
\end{aligned}
$$

Note that the divisor $\epsilon_{1}=0$ is not present in the Calabi-Yau threefold after the resolution. A subtlety here is that the divisor $\delta_{2}=0$ is reducible with two components, hence the total number of non-flat surface components is still 10 from $\delta_{i},(i=1, \cdots, 9)$.

The $\left(\mathrm{E}_{8}, \mathrm{E}_{8}\right)$ conformal matter theory is a $6 \mathrm{~d}(1,0)$ SCFT with rank 21 . The tensor branch configuration is

$$
\left[\mathrm{E}_{8}\right]-1-2-\stackrel{\mathfrak{s u}_{2}}{2}-\stackrel{\mathfrak{g}_{2}}{3}-1-\stackrel{\mathfrak{f}_{4}}{5}-1-\stackrel{\mathfrak{g}_{2}}{3}-\stackrel{\mathfrak{s u}_{2}}{2}-2-1-\left[\mathrm{E}_{8}\right] .
$$

Starting with the Weierstrass model:

$$
y^{2}+b_{1} U V x y+b_{3} U^{3} V^{3} y=x^{3}+b_{2} U^{2} V^{2} x^{2}+b_{4} U^{4} V^{4} x+b_{6} U^{5} V^{5},
$$

we first blow up the base locus $\left(U, V ; \delta_{1}\right)$, and then use the following resolution sequence:

$$
\begin{aligned}
\mathrm{BU}_{\text {marginal }}^{\left(\mathrm{E}_{8}, \mathrm{E}_{8}\right)} & = \\
\left\{\left\{x, y, U, u_{1}\right\},\left\{x, y, u_{1}, u_{2}\right\},\left\{y, u_{2}, u_{3}\right\},\left\{y, u_{1}, u_{3}, u_{4}\right\},\left\{y, u_{1}, u_{5}\right\},\left\{u_{1}, u_{3}, u_{6}\right\},\right. & \\
& \left\{u_{1}, u_{4}, u_{7}\right\},\left\{u_{1}, u_{5}, u_{8}\right\},\left\{u_{2}, u_{3}, u_{9}\right\},\left\{u_{3}, u_{4}, u_{10}\right\},\left\{u_{4}, u_{6}, u_{11}\right\},\left\{u_{3}, u_{6}, u_{12}\right\}, \\
& \left\{u_{6}, u_{10}, u_{13}\right\},\left\{u_{10}, u_{12}, u_{14}\right\},\left\{u_{3}, u_{12}, u_{15}\right\},\left\{x, y, V, v_{1}\right\},\left\{x, y, v_{1}, v_{2}\right\}, \\
& \left\{y, v_{2}, v_{3}\right\},\left\{y, v_{1}, v_{3}, v_{4}\right\},\left\{y, v_{1}, v_{5}\right\},\left\{v_{1}, v_{3}, v_{6}\right\},\left\{v_{1}, v_{4}, v_{7}\right\},\left\{v_{1}, v_{5}, v_{8}\right\}, \\
& \left\{v_{2}, v_{3}, v_{9}\right\},\left\{v_{3}, v_{4}, v_{10}\right\},\left\{v_{4}, v_{6}, v_{11}\right\},\left\{v_{3}, v_{6}, v_{12}\right\},\left\{v_{6}, v_{10}, v_{13}\right\},\left\{v_{10}, v_{12}, v_{14}\right\}, \\
& \left\{v_{3}, v_{12}, v_{15}\right\},\left\{u_{3}, \delta_{1}, \delta_{9}\right\},\left\{v_{3}, \delta_{1}, \delta_{6}\right\},\left\{u_{3}, \delta_{9}, \delta_{16}\right\},\left\{v_{3}, \delta_{6}, \delta_{14}\right\},\left\{u_{3}, \delta_{16}, \delta_{18}\right\}, \\
& \left\{v_{3}, \delta_{14}, \delta_{19}\right\},\left\{u_{3}, \delta_{18}, \delta_{21}\right\},\left\{v_{3}, \delta_{19}, \delta_{20}\right\},\left\{x, y, \delta_{1}, \epsilon_{1}\right\},\left\{x, y, \delta_{6}, \epsilon_{3}\right\}, \\
& \left\{y, \epsilon_{3}, \delta_{7}\right\},\left\{x, y, \delta_{9}, \epsilon_{4}\right\},\left\{y, \epsilon_{4}, \delta_{10}\right\},\left\{\epsilon_{1}, \delta_{7}, \delta_{12}\right\},\left\{x, y, \epsilon_{1}, \delta_{2}\right\}, \\
& \left\{y, \epsilon_{1}, \epsilon_{2}\right\},\left\{\epsilon_{1}, \delta_{10}, \delta_{13}\right\},\left\{\epsilon_{4}, \delta_{10}, \delta_{11}\right\},\left\{x, y, \delta_{14}, \delta_{15}\right\},\left\{\epsilon_{1}, \epsilon_{2}, \delta_{3}\right\}, \\
& \left.\left\{\delta_{2}, \epsilon_{2}, \delta_{4}\right\},\left\{\epsilon_{2}, \delta_{4}, \delta_{5}\right\},\left\{\epsilon_{3}, \delta_{7}, \delta_{8}\right\},\left\{x, y, \delta_{16}, \delta_{17}\right\}\right\} .
\end{aligned}
$$

Note that the nine non-flat fiber components are $\delta_{i}=0,(i=1, \cdots, 21)$, and the divisors $\epsilon_{i}=0, i=1, \cdots, 4$ are not present in the Calabi-Yau threefold after the resolution. 


\section{D.6 Alternative 6d starting points}

If we start with a $6 \mathrm{~d}$ SCFT, where the maximal superconformal flavor symmetry is not manifest, the descedant $5 \mathrm{~d}$ theories will not have manifest superconformal flavor symmetry either. We thus usually start with a $6 \mathrm{~d}$ model, which has manifestly the maximal $G_{\mathrm{F}}^{(6 d)}$. However it is interesting to see how the flavor symmetry enhancement emerges, when choosing different starting points in 6d. For rank one, an example is $\left(\mathrm{E}_{6}, \mathrm{SU}(3)\right)$ (instead of $\left.\left(\mathrm{E}_{8}, I_{1}\right)\right)$. Likewise in rank two, there is $\left(D_{k}, D_{k}\right)$ instead of $\left(D_{2 k}, I_{1}\right)$. In such cases the flavor symmetry is reconstructed in $5 \mathrm{~d}$ by considering the BPS states, which reorganize in terms of a larger superconformal flavor symmetry.

\section{D.6.1 Alternative description for rank one: $\left(\mathrm{E}_{6}, \mathrm{SU}(3)\right)$}

An alternative description of the rank one E-string theories can be obtained with the following starting point Tate model:

$$
y^{2}+b_{1} U x y+b_{3} U^{2} V y=x^{3}+b_{2} U^{2} V x^{2}+b_{4} U^{3} V^{2} x+b_{6} U^{5} V^{3} .
$$

There is an $\mathrm{E}_{6}$ on $U=0$ and $\mathrm{SU}(3)$ on $V=0$.

As an example, we can use the following blow-up sequence

$$
\begin{aligned}
& \mathrm{BU}^{\left(\mathrm{E}_{6}, \mathrm{SU}(3)\right)}= \\
& \quad\left\{\left\{x, y, U, u_{1}\right\},\left\{x, y, V, v_{1}\right\},\left\{y, v_{1}, v_{2}\right\},\left\{u_{1}, v_{2}, \delta_{1}\right\},\left\{x, y, u_{1}, u_{2}\right\},\right. \\
& \left.\quad\left\{y, u_{1}, u_{2}, u_{3}\right\},\left\{y, u_{1}, u_{4}\right\},\left\{y, u_{2}, u_{5}\right\},\left\{u_{3}, u_{4}, u_{6}\right\},\left\{y, u_{3}, u_{7}\right\},\left\{u_{1}, u_{4}, u_{8}\right\}\right\}
\end{aligned}
$$

The Cartan divisors $D_{i}^{\mathrm{E}_{6}}$ and $D_{i}^{\mathrm{SU}(3)}$ are given by

$$
\begin{aligned}
\left(D_{0}^{\mathrm{E}_{6}}, D_{1}^{\mathrm{E}_{6}}, \cdots, D_{6}^{\mathrm{E}_{6}}\right) & \equiv\left(U, u_{2}, u_{3}, u_{6}, u_{7}, u_{5}, u_{8}\right) \\
\left(D_{0}^{\mathrm{SU}(3)}, D_{1}^{\mathrm{SU}(3)}, D_{2}^{\mathrm{SU}(3)}\right) & \equiv\left(V, v_{1}, v_{2}\right)
\end{aligned}
$$

We plot the configuration of curves in figure 24 , and the reduced intersection matrix $S \cdot D_{i}^{2}$ is:

$$
\begin{array}{c|ccccccc|ccc|c}
S \cdot D_{i}^{2} & D_{0}^{\mathrm{E}_{6}} & D_{1}^{\mathrm{E}_{6}} & D_{2}^{\mathrm{E}_{6}} & D_{3}^{\mathrm{E}_{6}} & D_{4}^{\mathrm{E}_{6}} & D_{5}^{\mathrm{E}_{6}} & D_{6}^{\mathrm{E}_{6}} & D_{0}^{\mathrm{SU}(3)} & D_{1}^{\mathrm{SU}(3)} & D_{2}^{\mathrm{SU}(3)} & S \\
\hline D_{i} & 0 & -2 & -2 & -2 & -2 & -2 & -1 & 0 & -2 & -2 & 2
\end{array}
$$

The non-flat fiber $S$ is a $\mathrm{gdP}_{7}$, and one can see that the configuration of $(-2)$-curves form the Dynkin diagram of $\mathrm{SU}(3) \times \mathrm{SU}(6)$.

From the figure 24, we read off the following rational (-1)-curves corresponding to the highest weight of various representations of $\mathrm{SU}(3) \times \mathrm{SU}(6)$ :

$$
\begin{aligned}
e_{4}: & (\mathbf{1}, \mathbf{2 0}) \\
e_{7}: & (\mathbf{3}, \mathbf{6}) \\
h-e_{1}-e_{6}: & (\overline{\mathbf{3}}, \overline{\mathbf{6}}) .
\end{aligned}
$$

They exactly combine into the $\mathbf{5 6}$ representation of $\mathrm{E}_{7} \supset \mathrm{SU}(3) \times \mathrm{SU}(6)$. 


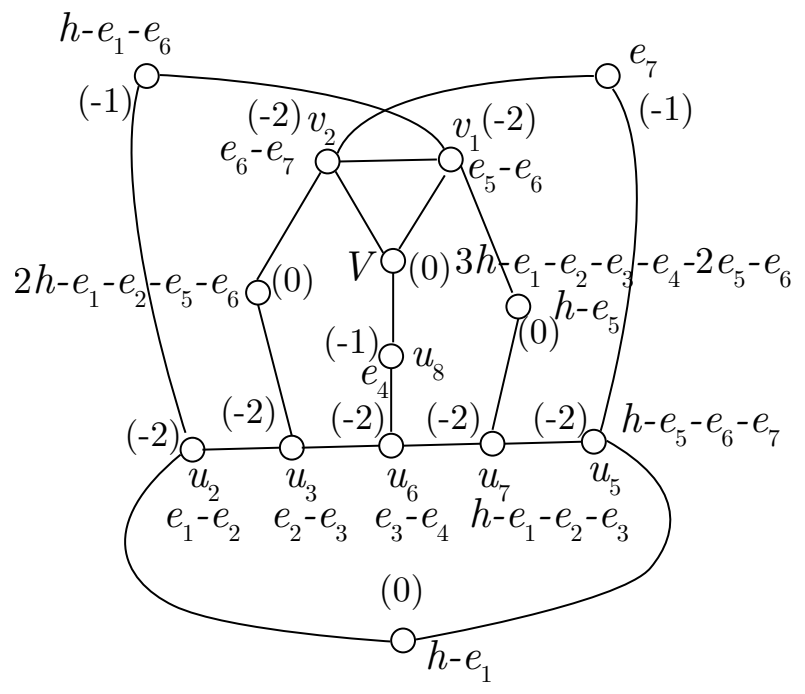

Figure 24. The configuration of curves in the example geometry $\mathrm{E}_{6} \times \mathrm{SU}(3)$, including the rational $(-2),(-1)$ and 0 -curves.

Moreover, the rational 0 -curves correspond to the highest weights of the following representations of $\mathrm{SU}(3) \times \mathrm{SU}(6)$ :

$$
\begin{aligned}
& h-e_{1}:(\mathbf{1}, \mathbf{3 5}) \\
& h-e_{5}:(\mathbf{3}, \overline{\mathbf{1 5}}) \\
& 2 h-e_{1}-e_{2}-e_{5}-e_{6}:(\overline{\mathbf{3}}, \mathbf{1 5}) \\
& 3 h-e_{1}-e_{2}-e_{3}-e_{4}-2 e_{5}-e_{6}:(\mathbf{8}, \mathbf{1}) .
\end{aligned}
$$

These representations can be exactly combined into the adjoint representation $\mathbf{1 3 3}$ of $\mathrm{E}_{7} \supset \mathrm{SU}(3) \times \mathrm{SU}(6)$. From this observation, we speculate that the actual flavor symmetry of this geometry is $\mathrm{E}_{7}$.

More generally, one can read off the actual non-abelian flavor symmetry $G_{F, n a}$ with the following steps:

- Read off the group $\tilde{G}_{F, n a}$ based on the configuration of $(-2)$-curves on the non-flat fiber, which form the Dynkin diagram of $\tilde{G}_{F, n a}$. The actual flavor symmetry group should contain $\tilde{G}_{F, n a}$.

- Write down all the irreducible (genus 0) curves $C_{i}$ on the non-flat fiber that gives rise to BPS states of a particular spin, see section 5 , which give rise to highest weight of various representations $R_{i}$ of $\tilde{G}_{F, n a}$.

- Check if the combined representation $\oplus_{i} R_{i}$ form an adjoint representation of a larger group $G_{F, n a}$. If this is the case, then the actual flavor symmetry group should be $G_{F, n a}$.

\section{D.6.2 Alternative description: $\left(D_{5}, D_{5}\right)$}

In the main text, we considered the starting point $D_{10}-I_{1}$ instead of the $\left(D_{5}, D_{5}\right)$ conformal matter. In fact it turns out that the former results in a more concise description of the 
theories, and contains the models descending from the latter. We illustrate now how the models with $\left(D_{5}, D_{5}\right)$ starting point give rise to the same theories as the $D_{10}$ starting point.

The starting point Tate model is:

$$
y^{2}+b_{1} U V x y+b_{3} U^{2} V^{2} y=x^{3}+b_{2} U V x^{2}+b_{4} U^{3} V^{3} x+b_{6} U^{5} V^{5} .
$$

As an example, we can use the following blow-up sequence:

$$
\begin{aligned}
& \mathrm{BU}^{D_{5}-D_{5}}= \\
& \left\{\left\{x, y, U, V, \delta_{2}\right\},\left\{x, y, U, u_{1}\right\},\left\{x, y, u_{1}, u_{2}\right\},\left\{y, u_{1}, u_{2}, u_{3}\right\},\left\{y, u_{1}, u_{4}\right\},\left\{y, u_{2}, u_{5}\right\},\right. \\
& \left\{u_{1}, u_{4}, u_{6}\right\},\left\{x, y, V, v_{1}\right\},\left\{x, y, v_{1}, v_{2}\right\},\left\{y, v_{1}, v_{2}, v_{3}\right\},\left\{y, v_{1}, v_{4}\right\},\left\{y, v_{2}, v_{5}\right\} \\
& \left.\left\{v_{1}, v_{4}, v_{6}\right\},\left\{x, y, \delta_{2}, \delta_{1}\right\}\right\},
\end{aligned}
$$

where the first blow-up is a weighted blow-up:

$$
x \rightarrow x \delta_{2}^{2}, y \rightarrow y \delta_{2}^{3}, U \rightarrow U \delta_{2}, V \rightarrow V \delta_{2} .
$$

The Cartan divisors $D_{i}^{(1)}$ and $D_{i}^{(2)}$ of the two $\mathrm{SO}(10)$ factors are given by

$$
\begin{aligned}
& \left(D_{0}^{(1)}, D_{1}^{(1)}, D_{2}^{(1)}, D_{3}^{(1)}, D_{4}^{(1)}, D_{5}^{(1)}\right) \equiv\left(U, u_{4}, u_{6}, u_{3}, u_{2}, u_{5}\right) \\
& \left(D_{0}^{(2)}, D_{1}^{(2)}, D_{2}^{(2)}, D_{3}^{(2)}, D_{4}^{(2)}, D_{5}^{(2)}\right) \equiv\left(V, v_{4}, v_{6}, v_{3}, v_{2}, v_{5}\right) .
\end{aligned}
$$

The reduced intersection matrix with the surface components $S_{i}$ is

$$
\begin{array}{c|cccccc|cccccc|cc}
S_{i} \cdot D_{j}^{2} & D_{0}^{(1)} & D_{1}^{(1)} & D_{2}^{(1)} & D_{3}^{(1)} & D_{4}^{(1)} & D_{5}^{(1)} & D_{0}^{(2)} & D_{1}^{(2)} & D_{2}^{(2)} & D_{3}^{(2)} & D_{4}^{(2)} & D_{5}^{(2)} & S_{1} & S_{2} \\
\hline S_{1} & 0 & 0 & 0 & 0 & 0 & 0 & 0 & 0 & 0 & 0 & 0 & 0 & 8 & 4 \\
S_{2} & -1 & -2 & -2 & -2 & -2 & -2 & -1 & -2 & -2 & -2 & -2 & -2 & -6 & -1
\end{array} .
$$

We plot the configuration of curves in figure 25. The configuration of $(-2)$-curves form $H=\mathrm{SO}(10) \times \mathrm{SO}(10)$. Note that $S_{1}$ is still an $\mathbb{F}_{1}$ as the curve $S_{1} \cdot S_{2}$ corresponds to the divisor class $2 h$. The geometry only has $\operatorname{Sp}(2)$ gauge theory description as any ruling on $S_{1}$ intersects $S_{2} \cdot S_{1}$ at two points. The following (-1)-curves on $S_{2}$ give rise to the highest weight of various representations under $\mathrm{SO}(10) \times \mathrm{SO}(10)$ :

$$
\begin{array}{ll}
h-e_{1}-e_{2}: & (\mathbf{4 5}, \mathbf{1}) \\
h-e_{6}-e_{7}: & (\mathbf{1}, \mathbf{4 5}) \\
h-e_{1}-e_{6}: & (\mathbf{1 0}, \mathbf{1 0}) .
\end{array}
$$

These representations can be combined into the adjoint representation $\mathbf{1 9 0}$ of $\mathrm{SO}(20)$. Hence we confirmed that the flavor symmetry of this configuration is $\mathrm{SO}(20)$.

\section{D.6.3 Flavor symmetry and BPS states}

What we have seen so far in the last subsections is that BPS states can help identify larger flavor symmetries. This is particularly important, if the marginal theory from which one starts the CFD-trees does not have the manifest flavor symmetry. 


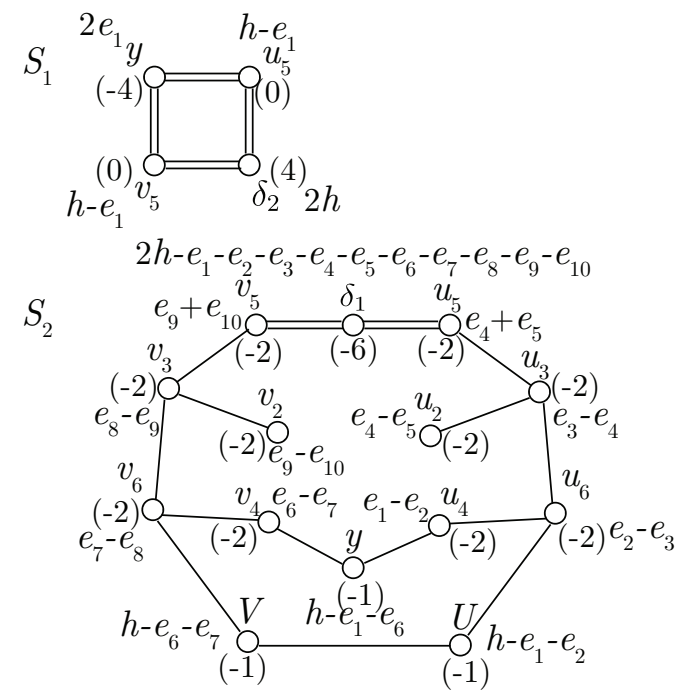

Figure 25. The configuration of curves on $S_{1}$ and $S_{2}$ in the geometry (D.88). The number in the bracket denotes the self-intersection number of the curve. The letters $U, V, u_{i}, v_{i}$, denote an intersection curve with the corresponding divisor.

To determine the superconformal flavor symmetry from the CFD, in the approach as laid out in the rest of this paper, it is important, nay vital, to begin with the correct marginal CFD. This marginal CFD is obtained by considering a particular resolution of singularities of a Weierstrass model which realizes, over a non-compact locus, a singular fiber associated to the affine Dynkin diagram of $G_{6 \mathrm{~d}}$, the superconformal flavor symmetry in $6 \mathrm{~d}$.

When attempting to determine the descendant $5 \mathrm{~d} \mathcal{N}=1$ SCFTs that arise from $6 \mathrm{~d}$ $\left(G, G^{\prime}\right)$ conformal matter one naturally begins with a Weierstrass model that contains two non-compact divisors in the base, which respectively support singular fibers associated to $G$ and $G^{\prime}$. However it is often the case that the superconformal flavour symmetry

$$
G_{6 \mathrm{~d}} \supseteq G \times G^{\prime}
$$

is not the product, but involves a non-trivial recombination of the two flavor symmetry factors - as in the $\left(\mathrm{E}_{6}, \mathrm{SU}(3)\right)$ and $\left(D_{5}, D_{5}\right)$ examples we studied. When this occurs one must first determine this enhancement before being able to determine the marginal CFD, to which the full flavor symmetry of the descendants is visible. An example of this that we have studied in this paper is the rank two theories that come from $\left(D_{5}, D_{5}\right)$ minimal conformal matter. In that case the 6 d flavor symmetry is not $\mathrm{SO}(10) \times \mathrm{SO}(10)$, but, in fact, $\mathrm{SO}(20)$, and if one were to write down the marginal CFD by considering a resolution of the Weierstrass model with $\mathrm{SO}(10) \times \mathrm{SO}(10)$, one would not fully capture the flavor symmetry in the CFDs.

Despite this, the full flavor symmetry is still observable, even if one begins with a marginal CFD not realizing $G_{6 \mathrm{~d}}$, from a study of the BPS spectra. In this section we will demonstrate this in several examples.

Let us begin by looking at the aforementioned example of $\left(D_{5}, D_{5}\right)$ minimal conformal matter. If one begins with an elliptic fibration with two $I_{1}^{*}$ fibers intersecting at a codi- 


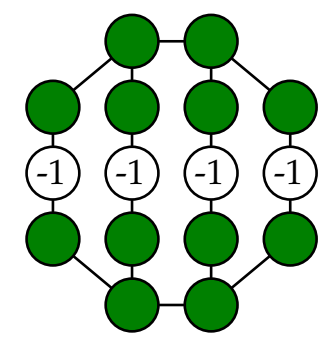

(a)

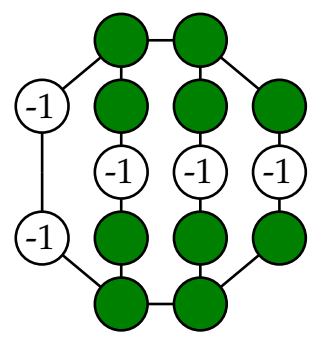

(b)

Figure 26. The marginal CFD for $\left(D_{5}, D_{5}\right)$ minimal conformal matter as determined from the Weierstrass model realizing intersecting $\left(I_{1}^{*}, I_{1}^{*}\right)$ singular fibers is shown on the left. On the right is the first descendant by taking a single mass deformation of the marginal CFD.

mension two point then constructing the non-flat resolution of that fibration would give rise to the marginal CFD as depicted in figure 26 a). Let us consider the first descendant of this CFD, obtainable by a single (unique) mass deformation from the marginal theory. From the CFD in figure $26 \mathrm{~b}$ ) one can observe an $\mathrm{SO}(10) \times \mathrm{SO}(10)$ flavor symmetry for the $5 \mathrm{~d}$ theory, however we know that this theory in fact has an $\mathrm{SO}(20)$ flavor symmetry.

If we determine the BPS states from this descendant CFD we can see that the spin 0 BPS states, form representations with the highest weights given by the vertices labelled with -1 . In terms of the $\mathrm{SO}(10) \times \mathrm{SO}(10)$ flavor group these form the representations

$$
(10,10),(16,16),\left(16^{\prime}, 16^{\prime}\right),(1,45),(45,1)
$$

If we consider the branching

$$
\mathrm{SO}(20) \rightarrow \mathrm{SO}(10) \times \mathrm{SO}(10)
$$

then one can see that the relevant representations decompose as

$$
\begin{aligned}
& 190 \rightarrow(45,1) \oplus(\mathbf{1}, 45) \oplus(10,10) \\
& 512 \rightarrow(16,16) \oplus\left(16^{\prime}, 16^{\prime}\right) .
\end{aligned}
$$

In this way one can see that there is, in fact, an enhanced $\mathrm{SO}(20)$ flavor symmetry under which the states transform. One can do a similar analysis for all descendant states from the $\left(I_{1}^{*}, I_{1}^{*}\right)$ starting point and observe that the BPS states from $g=0$ curves at the spin 0 and spin 1 level always form themselves into representations that can be combined into full representations of the superconformal flavor symmetry as given by the $\left(I_{6}^{*}, I_{1}\right)$ marginal CFD.

Open Access. This article is distributed under the terms of the Creative Commons Attribution License (CC-BY 4.0), which permits any use, distribution and reproduction in any medium, provided the original author(s) and source are credited. 


\section{References}

[1] E. Witten, Some comments on string dynamics, in the proceedings of Future perspectives in string theory (Strings'95), March 13-18, Los Angeles, U.S.A. (1995), hep-th/9507121 [INSPIRE].

[2] J.J. Heckman, D.R. Morrison and C. Vafa, On the classification of $6 D$ SCFTs and generalized ADE orbifolds, JHEP 05 (2014) 028 [Erratum ibid. 06 (2015) 017] [arXiv: 1312.5746] [INSPIRE].

[3] L. Bhardwaj, Classification of $6 d \mathcal{N}=(1,0)$ gauge theories, JHEP 11 (2015) 002 [arXiv: 1502.06594] [INSPIRE].

[4] J.J. Heckman, D.R. Morrison, T. Rudelius and C. Vafa, Atomic classification of $6 D$ SCFTs, Fortsch. Phys. 63 (2015) 468 [arXiv: 1502.05405] [InSPIRE].

[5] K.A. Intriligator, D.R. Morrison and N. Seiberg, Five-dimensional supersymmetric gauge theories and degenerations of Calabi-Yau spaces, Nucl. Phys. B 497 (1997) 56 [hep-th/9702198] [INSPIRE].

[6] P. Jefferson, S. Katz, H.-C. Kim and C. Vafa, On geometric classification of 5d SCFTs, JHEP 04 (2018) 103 [arXiv: 1801.04036] [INSPIRE].

[7] P. Argyres, M. Lotito, Y. Lü and M. Martone, Geometric constraints on the space of $\mathcal{N}=2$ SCFTs. Part I. Physical constraints on relevant deformations, JHEP 02 (2018) 001 [arXiv: 1505.04814] [INSPIRE].

[8] F. Apruzzi et al., 5d superconformal field theories and graphs, arXiv:1906.11820 [INSPIRE].

[9] D.R. Morrison and N. Seiberg, Extremal transitions and five-dimensional supersymmetric field theories, Nucl. Phys. B 483 (1997) 229 [hep-th/9609070] [INSPIRE].

[10] D. Xie and S.-T. Yau, Three dimensional canonical singularity and five dimensional $\mathcal{N}=1$ SCFT, JHEP 06 (2017) 134 [arXiv:1704.00799] [INSPIRE].

[11] M. Del Zotto, J.J. Heckman and D.R. Morrison, 6D SCFTs and phases of 5D theories, JHEP 09 (2017) 147 [arXiv:1703.02981] [INSPIRE].

[12] P. Jefferson, H.-C. Kim, C. Vafa and G. Zafrir, Towards classification of 5d SCFTs: single gauge node, arXiv:1705.05836 [INSPIRE].

[13] F. Apruzzi, L. Lin and C. Mayrhofer, Phases of 5d SCFTs from M-/F-theory on non-flat fibrations, JHEP 05 (2019) 187 [arXiv: 1811.12400] [INSPIRE].

[14] C. Closset, M. Del Zotto and V. Saxena, Five-dimensional SCFTs and gauge theory phases: an M-theory/type IIA perspective, SciPost Phys. 6 (2019) 052 [arXiv:1812.10451] [INSPIRE].

[15] O. Aharony and A. Hanany, Branes, superpotentials and superconformal fixed points, Nucl. Phys. B 504 (1997) 239 [hep-th/9704170] [INSPIRE].

[16] O. Aharony, A. Hanany and B. Kol, Webs of $(p, q)$ five-branes, five-dimensional field theories and grid diagrams, JHEP 01 (1998) 002 [hep-th/9710116] [INSPIRE].

[17] O. DeWolfe, A. Hanany, A. Iqbal and E. Katz, Five-branes, seven-branes and five-dimensional E(n) field theories, JHEP 03 (1999) 006 [hep-th/9902179] [INSPIRE].

[18] O. Bergman and G. Zafrir, 5d fixed points from brane webs and O7-planes, JHEP 12 (2015) 163 [arXiv: 1507.03860] [INSPIRE]. 
[19] G. Zafrir, Brane webs, 5d gauge theories and $6 d \mathcal{N}=(1,0)$ SCFT's, JHEP 12 (2015) 157 [arXiv: 1509.02016] [INSPIRE].

[20] G. Zafrir, Brane webs and O5-planes, JHEP 03 (2016) 109 [arXiv:1512.08114] [INSPIRE].

[21] K. Ohmori and H. Shimizu, $S^{1} / T^{2}$ compactifications of $6 d \mathcal{N}=(1,0)$ theories and brane webs, JHEP 03 (2016) 024 [arXiv:1509.03195] [INSPIRE].

[22] H. Hayashi, S.-S. Kim, K. Lee and F. Yagi, Discrete theta angle from an O5-plane, JHEP 11 (2017) 041 [arXiv: 1707.07181] [InSPIRE].

[23] H. Hayashi, S.-S. Kim, K. Lee and F. Yagi, 5-brane webs for $5 d \mathcal{N}=1 G_{2}$ gauge theories, JHEP 03 (2018) 125 [arXiv: 1801.03916] [INSPIRE].

[24] H. Hayashi, S.-S. Kim, K. Lee and F. Yagi, Dualities and 5-brane webs for $5 d$ rank 2 SCFTs, JHEP 12 (2018) 016 [arXiv: 1806.10569] [INSPIRE].

[25] N. Seiberg, Five-dimensional SUSY field theories, nontrivial fixed points and string dynamics, Phys. Lett. B 388 (1996) 753 [hep-th/9608111] [INSPIRE].

[26] A. Brandhuber and Y. Oz, The D4-D8 brane system and five-dimensional fixed points, Phys. Lett. B 460 (1999) 307 [hep-th/9905148] [INSPIRE].

[27] O. Bergman and D. Rodriguez-Gomez, 5d quivers and their AdS 6 duals, JHEP 07 (2012) 171 [arXiv: 1206.3503] [INSPIRE].

[28] H. Hayashi, C. Lawrie, D.R. Morrison and S. Schäfer-Nameki, Box graphs and singular fibers, JHEP 05 (2014) 048 [arXiv: 1402.2653] [INSPIRE].

[29] J. Tian and Y.-N. Wang, E-string spectrum and typical F-theory geometry, arXiv: 1811.02837 [INSPIRE].

[30] F. Apruzzi et al., Fibers add flavor. Part III. Higher rank,

[31] F. Apruzzi et al., Fibers add flavor. Part II. 5d SCFTs, gauge theories and dualities, arXiv: 1909.09128 [INSPIRE].

[32] H.-C. Kim, S.-S. Kim and K. Lee, 5-dim superconformal index with enhanced en global symmetry, JHEP 10 (2012) 142 [arXiv:1206.6781] [INSPIRE].

[33] O. Bergman, D. Rodríguez-Gómez and G. Zafrir, 5-brane webs, symmetry enhancement and duality in 5d supersymmetric gauge theory, JHEP 03 (2014) 112 [arXiv:1311.4199] [INSPIRE].

[34] G. Zafrir, Duality and enhancement of symmetry in 5d gauge theories, JHEP 12 (2014) 116 [arXiv: 1408.4040] [INSPIRE].

[35] V. Mitev, E. Pomoni, M. Taki and F. Yagi, Fiber-base duality and global symmetry enhancement, JHEP 04 (2015) 052 [arXiv: 1411.2450] [INSPIRE].

[36] C. Hwang, J. Kim, S. Kim and J. Park, General instanton counting and 5d SCFT, JHEP 07 (2015) 063 [arXiv:1406.6793] [INSPIRE].

[37] D. Gaiotto and H.-C. Kim, Duality walls and defects in $5 d \mathcal{N}=1$ theories, JHEP 01 (2017) 019 [arXiv: 1506.03871] [INSPIRE].

[38] Y. Tachikawa, Instanton operators and symmetry enhancement in 5d supersymmetric gauge theories, PTEP 2015 (2015) 043B06 [arXiv:1501.01031] [INSPIRE].

[39] K. Yonekura, Instanton operators and symmetry enhancement in $5 d$ supersymmetric quiver gauge theories, JHEP 07 (2015) 167 [arXiv: 1505. 04743] [INSPIRE]. 
[40] G. Zafrir, Instanton operators and symmetry enhancement in 5d supersymmetric USp, SO and exceptional gauge theories, JHEP 07 (2015) 087 [arXiv:1503.08136] [INSPIRE].

[41] O. Bergman and D. Rodriguez-Gomez, A note on instanton operators, instanton particles and supersymmetry, JHEP 05 (2016) 068 [arXiv:1601.00752] [INSPIRE].

[42] H. Hayashi et al., A new $5 d$ description of $6 d$ D-type minimal conformal matter, JHEP 08 (2015) 097 [arXiv : 1505.04439] [INSPIRE].

[43] G. Ferlito, A. Hanany, N. Mekareeya and G. Zafrir, 3d Coulomb branch and $5 d$ Higgs branch at infinite coupling, JHEP 07 (2018) 061 [arXiv:1712.06604] [INSPIRE].

[44] S. Cabrera, A. Hanany and F. Yagi, Tropical geometry and five dimensional Higgs branches at infinite coupling, JHEP 01 (2019) 068 [arXiv:1810.01379] [INSPIRE].

[45] M. Del Zotto, J.J. Heckman, A. Tomasiello and C. Vafa, 6d conformal matter, JHEP 02 (2015) 054 [arXiv: 1407.6359] [INSPIRE].

[46] C. Lawrie and S. Schäfer-Nameki, The Tate form on steroids: resolution and higher codimension fibers, JHEP 04 (2013) 061 [arXiv:1212.2949] [INSPIRE].

[47] P. Candelas et al., Codimension three bundle singularities in F-theory, JHEP 06 (2002) 014 [hep-th/0009228] [INSPIRE].

[48] V. Braun, Toric elliptic fibrations and F-theory compactifications, JHEP 01 (2013) 016 [arXiv: 1110.4883] [INSPIRE].

[49] V. Braun, T.W. Grimm and J. Keitel, Geometric engineering in toric F-theory and GUTs with U(1) gauge factors, JHEP 12 (2013) 069 [arXiv: 1306.0577] [INSPIRE].

[50] M. Cvetič, A. Grassi, D. Klevers and H. Piragua, Chiral four-dimensional f-theory compactifications with $\mathrm{SU}(5)$ and multiple $\mathrm{U}(1)$-factors, JHEP 04 (2014) 010 [arXiv: 1306.3987] [INSPIRE].

[51] F. Baume, E. Palti and S. Schwieger, On $\mathrm{E}_{8}$ and F-theory GUTs, JHEP 06 (2015) 039 [arXiv: 1502.03878] [INSPIRE].

[52] L.B. Anderson, X. Gao, J. Gray and S.-J. Lee, Tools for CICYs in F-theory, JHEP 11 (2016) 004 [arXiv: 1608.07554] [INSPIRE].

[53] W. Buchmüller, M. Dierigl, P.-K. Oehlmann and F. Ruehle, The toric $\mathrm{SO}(10)$ F-theory landscape, JHEP 12 (2017) 035 [arXiv:1709.06609] [INSPIRE].

[54] L.B. Anderson, X. Gao, J. Gray and S.-J. Lee, Fibrations in CICY threefolds, JHEP 10 (2017) 077 [arXiv: 1708.07907] [inSPIRE].

[55] Y.-C. Huang and W. Taylor, Comparing elliptic and toric hypersurface Calabi-Yau threefolds at large Hodge numbers, JHEP 02 (2019) 087 [arXiv: 1805.05907] [INSPIRE].

[56] M. Dierigl, P.-K. Oehlmann and F. Ruehle, Global tensor-matter transitions in F-theory, Fortsch. Phys. 66 (2018) 1800037 [arXiv:1804.07386] [INSPIRE].

[57] I. Achmed-Zade, I. García-Etxebarria and C. Mayrhofer, A note on non-flat points in the $\mathrm{SU}(5) \times \mathrm{U}(1)_{P Q}$ F-theory model, JHEP 05 (2019) 013 [arXiv: 1806.05612] [INSPIRE].

[58] R. Miranda, Smooth models for elliptic threefolds, in The birational geometry of degenerations, R. Friedman ed., Progress in Mathematics volume 29, Birkhäuser, Boston U.S.A. (1983). 
[59] M. Esole, M.J. Kang and S.-T. Yau, Mordell-Weil torsion, anomalies and phase transitions, arXiv: 1712.02337 [INSPIRE].

[60] M. Esole, R. Jagadeesan and M.J. Kang, 48 Crepant paths to $\mathrm{SU}(2) \times \mathrm{SU}(3)$, arXiv: 1905.05174 [INSPIRE].

[61] M. Esole and M.J. Kang, Flopping and slicing: $\mathrm{SO}(4)$ and $\mathrm{Spin}(4)$-models, arXiv: 1802.04802 [INSPIRE].

[62] M. Esole and M.J. Kang, The geometry of the $\mathrm{SU}(2) \times G_{2}$-model, JHEP 02 (2019) 091 [arXiv: 1805.03214] [INSPIRE].

[63] M. Bertolini, P.R. Merkx and D.R. Morrison, On the global symmetries of $6 D$ superconformal field theories, JHEP 07 (2016) 005 [arXiv: 1510.08056] [INSPIRE].

[64] P.R. Merkx, Classifying global symmetries of 6D SCFTs, JHEP 03 (2018) 163 [arXiv: 1711.05155] [INSPIRE].

[65] A.P. Braun and S. Schäfer-Nameki, Box graphs and resolutions I, Nucl. Phys. B 905 (2016) 447 [arXiv: 1407.3520] [INSPIRE].

[66] A.P. Braun and S. Schäfer-Nameki, Box graphs and resolutions II: from Coulomb phases to fiber faces, Nucl. Phys. B 905 (2016) 480 [arXiv:1511.01801] [InSPIRE].

[67] C. Lawrie, S. Schäfer-Nameki and J.-M. Wong, F-theory and all things rational: surveying U(1) symmetries with rational sections, JHEP 09 (2015) 144 [arXiv:1504.05593] [INSPIRE].

[68] O.J. Ganor, D.R. Morrison and N. Seiberg, Branes, Calabi-Yau spaces and toroidal compactification of the $N=1$ six-dimensional $\mathrm{E}_{8}$ theory, Nucl. Phys. B 487 (1997) 93 [hep-th/9610251] [INSPIRE].

[69] A.C. Cadavid, A. Ceresole, R. D'Auria and S. Ferrara, Eleven-dimensional supergravity compactified on Calabi-Yau threefolds, Phys. Lett. B 357 (1995) 76 [hep-th/9506144] [INSPIRE].

[70] S. Ferrara, R.R. Khuri and R. Minasian, $M$ theory on a Calabi-Yau manifold, Phys. Lett. B 375 (1996) 81 [hep-th/9602102] [INSPIRE].

[71] E. Witten, Phase transitions in M-theory and F-theory, Nucl. Phys. B 471 (1996) 195 [hep-th/9603150] [INSPIRE].

[72] S. Ferrara, R. Minasian and A. Sagnotti, Low-energy analysis of $M$ and $F$ theories on Calabi-Yau threefolds, Nucl. Phys. B 474 (1996) 323 [hep-th/9604097] [INSPIRE].

[73] L. Bhardwaj and P. Jefferson, Classifying 5d SCFTs via 6d SCFTs: rank one, JHEP 07 (2019) 178 [arXiv:1809.01650] [INSPIRE].

[74] L. Bhardwaj and P. Jefferson, Classifying 5d SCFTs via 6d SCFTs: arbitrary rank, arXiv:1811.10616 [INSPIRE].

[75] C. Vafa, Evidence for F-theory, Nucl. Phys. B 469 (1996) 403 [hep-th/9602022] [InSPIRE].

[76] D.R. Morrison and C. Vafa, Compactifications of F-theory on Calabi-Yau threefolds. 1, Nucl. Phys. B 473 (1996) 74 [hep-th/9602114] [INSPIRE].

[77] D.R. Morrison and C. Vafa, Compactifications of F-theory on Calabi-Yau threefolds. 2, Nucl. Phys. B 476 (1996) 437 [hep-th/9603161] [INSPIRE].

[78] S.H. Katz and C. Vafa, Matter from geometry, Nucl. Phys. B 497 (1997) 146 [hep-th/9606086] [INSPIRE]. 
[79] M. Bershadsky et al., Geometric singularities and enhanced gauge symmetries, Nucl. Phys. B 481 (1996) 215 [hep-th/9605200] [INSPIRE].

[80] S. Katz, D.R. Morrison, S. Schäfer-Nameki and J. Sully, Tate's algorithm and F-theory, JHEP 08 (2011) 094 [arXiv:1106.3854] [INSPIRE].

[81] D.S. Park, Anomaly equations and intersection theory, JHEP 01 (2012) 093 [arXiv:1111.2351] [INSPIRE].

[82] M. Esole and S.-T. Yau, Small resolutions of SU(5)-models in F-theory, Adv. Theor. Math. Phys. 17 (2013) 1195 [arXiv:1107.0733] [inSPIRE].

[83] J. Marsano and S. Schäfer-Nameki, Yukawas, G-flux and spectral covers from resolved Calabi-Yau's, JHEP 11 (2011) 098 [arXiv:1108.1794] [INSPIRE].

[84] S. Krause, C. Mayrhofer and T. Weigand, $G_{4}$ flux, chiral matter and singularity resolution in F-theory compactifications, Nucl. Phys. B 858 (2012) 1 [arXiv:1109.3454] [InSPIRE].

[85] M. Esole, P. Jefferson and M.J. Kang, Euler characteristics of crepant resolutions of Weierstrass models, Commun. Math. Phys. 371 (2019) 99 [arXiv:1703.00905] [InSPIRE].

[86] N. Mekareeya, K. Ohmori, Y. Tachikawa and G. Zafrir, $\mathrm{E}_{8}$ instantons on type-A ALE spaces and supersymmetric field theories, JHEP 09 (2017) 144 [arXiv: 1707.04370] [INSPIRE].

[87] Y. Tachikawa, On S-duality of 5d super Yang-Mills on $S^{1}$, JHEP 11 (2011) 123 [arXiv:1110.0531] [INSPIRE].

[88] L. Bhardwaj, D.R. Morrison, Y. Tachikawa and A. Tomasiello, The frozen phase of F-theory, JHEP 08 (2018) 138 [arXiv: 1805.09070] [INSPIRE].

[89] F. Apruzzi et al., Supplementary material: CFD-trees, https://people.maths.ox.ac.uk/schafernamek/CFD/.

[90] R. Gopakumar and C. Vafa, M theory and topological strings. 2., hep-th/9812127 [INSPIRE].

[91] S. Kachru and M. Zimet, A comment on 4d and 5d BPS states, arXiv:1808.01529 [INSPIRE].

[92] M. Taki, Seiberg duality, 5D SCFTs and Nekrasov partition functions, arXiv:1401.7200 [INSPIRE].

[93] N. Yamatsu, Finite-dimensional Lie algebras and their representations for unified model building, arXiv:1511.08771 [INSPIRE].

[94] M.-X. Huang, A. Klemm and M. Poretschkin, Refined stable pair invariants for E-, M- and [p,q]-strings, JHEP 11 (2013) 112 [arXiv:1308.0619] [INSPIRE].

[95] S. Cecotti, D. Gaiotto and C. Vafa, tt* geometry in 3 and 4 dimensions, JHEP 05 (2014) 055 [arXiv: 1312.1008] [INSPIRE].

[96] S. Banerjee, P. Longhi and M. Romo, Exploring 5d BPS spectra with exponential networks, Annales Henri Poincaré 20 (2019) 4055 [arXiv: 1811.02875] [INSPIRE].

[97] U. Derenthal, Geometry of universal torsors, Ph.D. thesis, Universität Göttingen, Göttingen, Germany (2006).

[98] U. Derenthal, Singular del pezzo surfaces whose universal torsors are hypersurfaces, Proc. London Math. Soc. 108 (2014) 638.

[99] W. Taylor and Y.-N. Wang, Non-toric bases for elliptic Calabi-Yau threefolds and $6 D$ F-theory vacua, Adv. Theor. Math. Phys. 21 (2017) 1063 [arXiv:1504.07689] [InSPIRE]. 